\begin{tabular}{|c|c|}
\hline $\begin{array}{l}\text { 2. To: (Receiving organization) } \\
\text { Distribution }\end{array}$ & $\begin{array}{l}\text { 3. From: (Originating Organization) } \\
\text { Numatec Hanford Corporation }\end{array}$ \\
\hline $\begin{array}{l}\text { 5. Proj./Prog./Dept./Div.: } \\
\text { Retrieval }\end{array}$ & $\begin{array}{l}\text { 6. Design Authority/ Design Agent/Cog. } \\
\text { Engr.: } \\
\text { A. F. Manuel }\end{array}$ \\
\hline
\end{tabular}

8. originator Remarks:

For approval and release.

11. Receiver Remarks: 11A. Design Baseline Document? [] Yes [x] No
4. Related EDT No.:

NA

7. Purchase Order No.:

NA

9. Equip./Component No.: NA

10. System/Bldg./Facility: NA

12. Major Assm. Dwg. No.: NA

13. Permit/Permit Application No.: NA

14. Required Response Date:

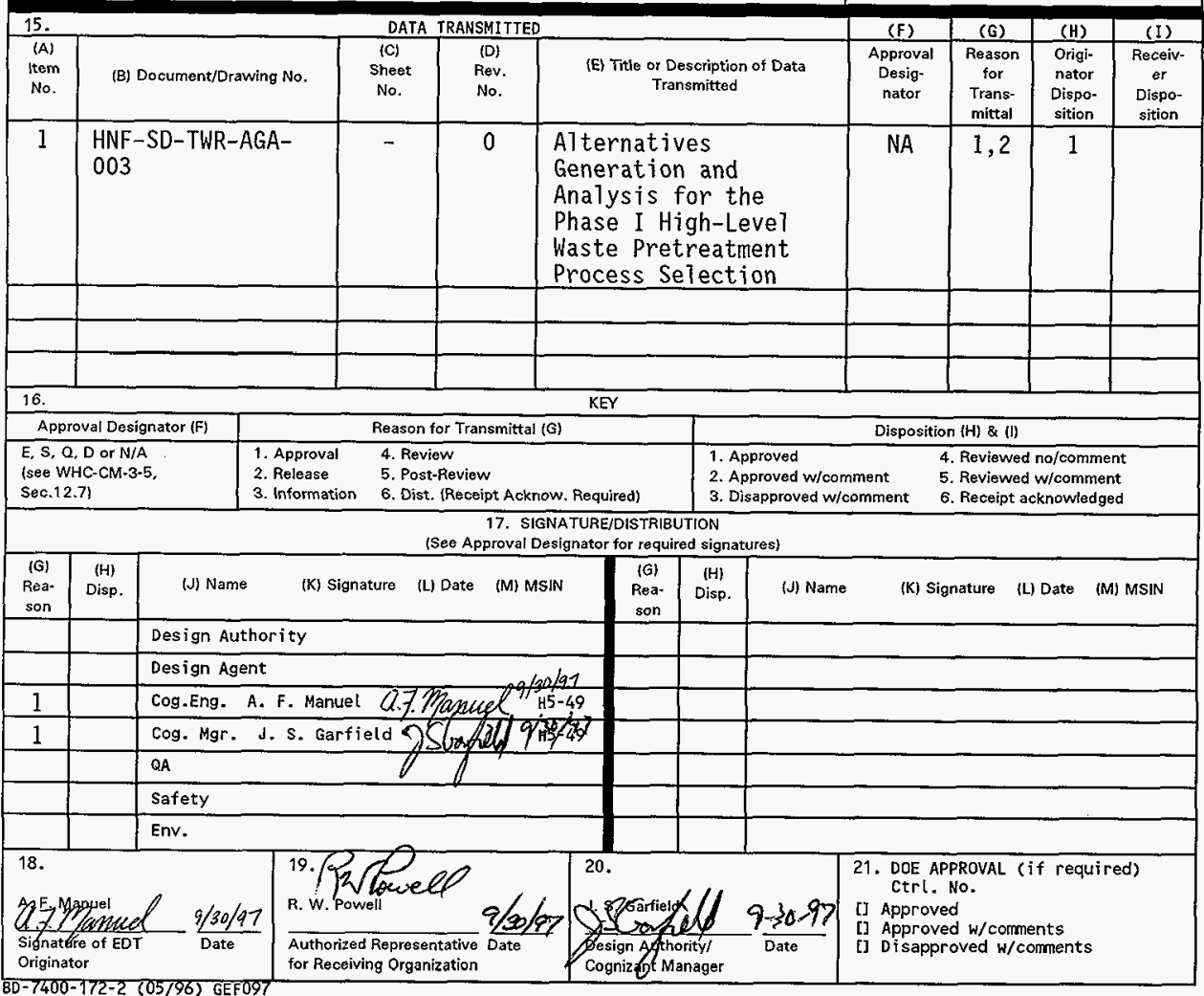




\title{
Alternatives Generation and Analysis for the Phase I High-Level Waste Pretreatment Process Selection
}

\author{
A. F. Manuel \\ Numatec Hanford Corporation, Richland, WA 99352 \\ U.S. Department of Energy Contract DE-AC06-96RL13200 \\ EDT/ECN: 622692 \\ UC: 721 \\ Org Code: $8 \mathrm{C} 451$ \\ Charge Code: D6229 \\ B\&R Code: EW3130010 \\ Total Pages: 167 cw $10-2.97$
}

Key Words: AGA, high-level waste, Phase I, pretreatment, enhanced sludge washing

Abstract: This report evaluates the effects of enhanced sludge washing and sludge washing (without caustic Teaching) during the preparation of the Phase I high-level waste feeds. The pretreatment processing alternatives are evaluated against their ability to satisfy contractual, cost minimization, and other criteria. The information contained in this report is consistent with, and supplemental to, the Tank Waste Remediation System Operation and Utilization Plan (Kirkbride et al. 1997).

TRADEMARK DISCLAIMER. Reference herein to any specific comercial product, process, or service by trade name, trademark, manufacturer, or otherwise, does not necessarily constitute or imply its endorsement, recomendation, or favoring by the United States Government or any agency thereof or its contractors or subcontractors.

Printed in the United States of America. To obtain copies of this document, contact: Document Control Services, P.0. Box 950, Mailstop H6-08, Richland WA 99352, Phone (509) 372-2420; Fax (509) 376-4989.
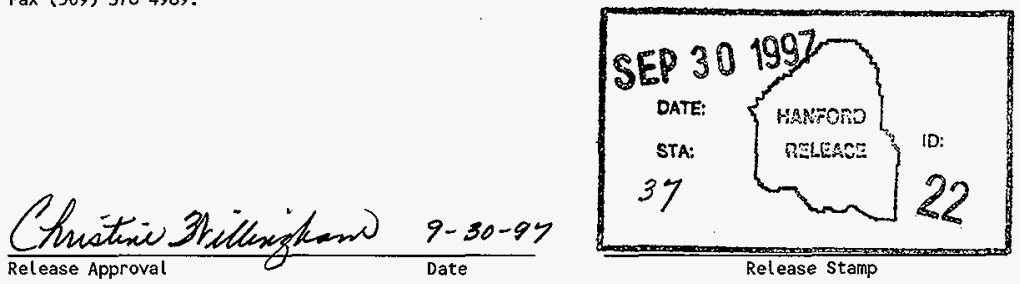

\section{Approved for Public Release}


HNF-SD-TWR-AGA-003

Revision 0

\title{
ALTERNATIVES GENERATION AND ANALYSIS FOR THE PHASE I HIGH-LEVEL WASTE PRETREATMENT PROCESS SELECTION
}

September 1997

\author{
A. F. Manuel \\ Numatec Hanford Corporation \\ Richland, Washington
}

Prepared for:

U.S. Department of Energy

Richland, Washington 
HNF-SD-TWR-AGA-003

Revision 0

\section{ACKNOWLEDGMENTS}

The author would like to thank the following personnel for their valuable assistance in completing this evaluation: John S. Garfield and Randy A. Kirkbride for their guidance and technical direction, Graham T. MacLean for performing the Environmental Simulation Program calculations, and Eric J. Slaathaug for performing the Hanford Tank Waste Operation Simulator runs to evaluate the effects of each alternative on tank farm operations. 
HNF-SD-TWR-AGA-003

Revision 0

EXECUTIVE SUMMARY

This report evaluates eight different pretreatment processing alternatives for the preparation of the Phase I high-level waste feeds against their ability to satisfy contractual, cost minimization, and other criteria. The alternatives are summarized in Table ES-1.

Table ES-1. Phase I High-Level Waste Pretreatment Processing Alternatives.

\begin{tabular}{|c|c|c|c|c|c|c|c|c|}
\hline & \multicolumn{8}{|c|}{ Alternatives } \\
\hline & 1 & 2 & 3 & 4 & 5 & 6 & 7 & 8 \\
\hline $241-A Z-101$ & $8 \mathrm{su}$ & $S W$ & 20.51\% & $S W$ & 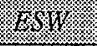 & 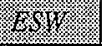 & $S W$ & $S W$ \\
\hline $241-A Z-102$ & 8.811 & $S W$ & 9811 & $4.817=$ & $S W$ & $S W$ & 18 & $S W$ \\
\hline $241-A Y-102^{a}$ & $2.5 \mathrm{st}$ & $S W$ & $S W$ & 4.918 & 18517 & $S W$ & $S W$ & $8.81 \%$ \\
\hline
\end{tabular}

${ }^{a} 241-A Y-102$ is assumed to contain 100 percent of the sludge from single-shell tank $241-C-106$.

$E S W=$ Indicates that the HLW feed is enhanced sludge washed

$H L W=$ High-level waste

$S W=$ Indicates that the HLW feed is sludge washed.

The primary findings of this evaluation are as follows.

- Any of the eight alternatives can be performed within the existing double-shell tank space, and a continuous high-level waste feed can be provided to the Private Contractor.

- To minimize the risk of non-compliance with the requirements and specifications established in the Phase IA Privatization contracts (but not necessarily to minimize life-cycle cost), Alternative 2 should be selected. 


\section{HNF-SD-TWR-AGA-003 \\ Revision 0}

- Based on the currently specified "Target-Unit-Prices" for high-level and lowactivity waste processing, the Phase I life-cycle costs are considerably lower for BNFL, Inc., as the high-level waste/low-activity waste contractor.

- Based on the currently specified BNFL, Inc. "Target-Unit-Prices"for high-level and low-activity waste processing, the pretreatment processing alternatives cannot be differentiated on the basis of life-cycle cost.

- Based on the currently specified LMAES "Target-Unit-Prices, "Alternative 1 is preferred on the basis of life-cycle cost.

To support the selection of the preferred pretreatment alternative based on life-cycle cost, the following information is needed but is not yet available:

- The "Fixed-Unit-Prices" for processing the minimum and maximum order quantities of high-level and low-activity waste (for each Private Contractor).

- The range and expected value of the immobilized high-level waste product loading based on an enhanced sludge washed feed and a sludge washed feed for each Private Contractor). 


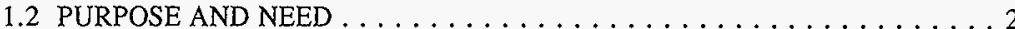

2.0 PHASE I HIGH-LEVEL WASTE FEED PREPARATION SUMMARY $\ldots \ldots \ldots \ldots 3$

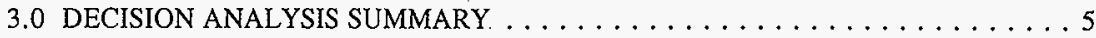

3.1 ALTERNATIVES GENERATION $\ldots \ldots \ldots \ldots \ldots \ldots \ldots \ldots \ldots$

3.1.1 High-Level Waste Pretreatment Processing Alternatives . . . . . . 6

3.1.2 High-Level Waste Pretreatment Equipment Alternatives . . . . . . . 14

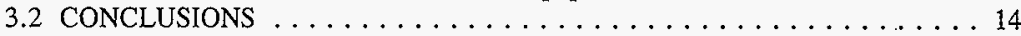

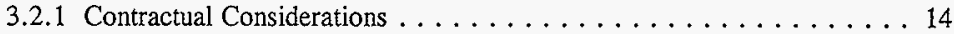

3.2.2 Cost Minimization Considerations $\ldots \ldots \ldots \ldots \ldots \ldots \ldots \ldots$

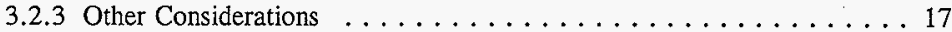

3.3 DESIGN REQUIREMENTS RECOMMENDATIONS $\ldots \ldots \ldots \ldots \ldots \ldots 18$

3.4 DESIGN REQUIREMENTS DECISION $\ldots \ldots \ldots \ldots \ldots \ldots \ldots$

4.0 PROBLEM STATEMENT . . . . . . . . . . . . . . . . . . . . . 19

4.1 SOURCE DOCUMENTS . . . . . . . . . . . . . . . . . . . 19

4.1.1 Tank Waste Remediation System Phase I Privatization Contracts

DE-RP06-96RL13308 (BNFL, Inc.) and DE-RP06-96RL13309

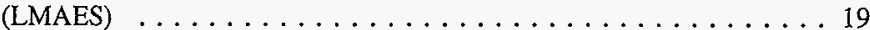

4.1.2 Tank Waste Remediation System Operation and Utilization Plan . . . . 20

4.1.3 Computer Simulation of the Leaching and Washing of Waste in Tanks 241-C-106, 241-AY-102, 241-AZ-101, and 241-AZ-102 . . . . . . 21

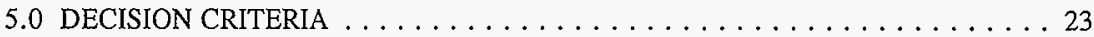

5.1 CONTRACTUAL CONSIDERATIONS ............. 23

5.1.1 Ability to Satisfy the Minimum Order Quantity of High-Level Waste

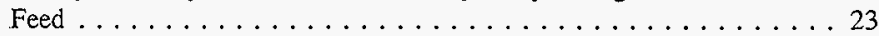

5.1.2 Ability to Satisfy the High-Level Waste Feed Specifications . . . . . . 24

5.1.3 Ability to Provide a Continuous High-Level Waste Feed . . . . . . . . 24

5.1.4 Ability to Satisfy the Low-Activity Waste Envelope B Specifications . . 24

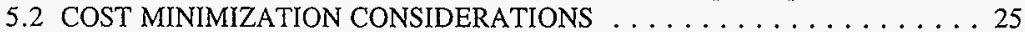

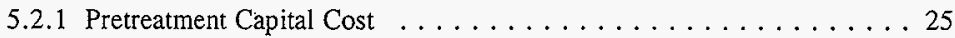

5.2.2 Pretreatment Operating Cost . . . . . . . . . . . . . 25

5.2.3 High-Level Waste Immobilization Cost (Minimum and Extended Order Quantities) ....................... 26

5.2.4 Low-Activity Waste Immobilization Cost (Extended Order Quantities) . 26

5.2.5 High-Level Waste Canister Interim Storage and Disposal Cost . . . . 27

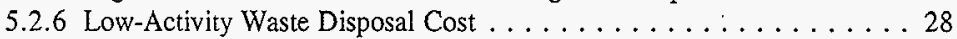

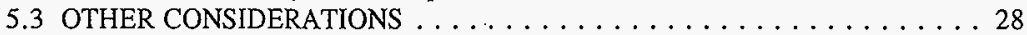




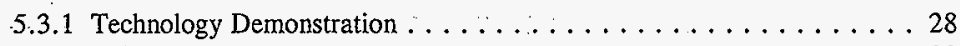

5.3 .2 Safety . . . . . . . . . . . . . . . . 29

6.0 ALTERNATIVES EVALUATION AND ANALYSIS $\ldots \ldots \ldots \ldots \ldots \ldots \ldots$

6.1 CONTRACTUAL CONSIDERATIONS . . . . . . . . . . . 31

6.1.1 Ability to Satisfy the Minimum Order Quantity of High-Level Waste

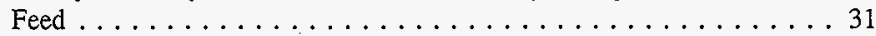

6.1.2 Ability to Satisfy the High-Level Waste Feed Specifications . . . . . . 32

6.1.3 Ability to Provide a Continuous High-Level Waste Feed . . . . . . . 35

6.1.4 Ability to Satisfy the Low-Activity Waste Envelope B Specifications . . 36

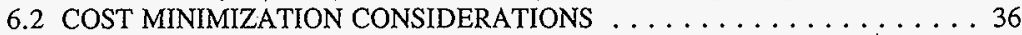

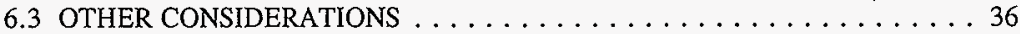

6.4 SUPPLEMENTAL INFORMATION $\ldots \ldots \ldots \ldots \ldots \ldots \ldots \ldots \ldots \ldots$

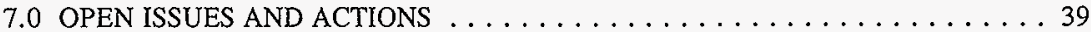

7.1 "FIXED-UNIT-PRICES" . . . . . . . . . . . . . . . . . . . 39

7.2 IMMOBILIZED HIGH-LEVEL WASTE PRODUCT VOLUME . . . . . . . 39

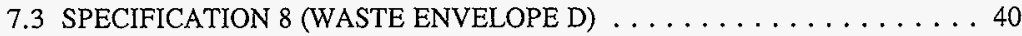

7.4 TANK SPACE IMPLICATIONS $\ldots \ldots \ldots \ldots \ldots \ldots \ldots \ldots \ldots \ldots \ldots$

7.5 HIGH-LEVEL WASTE PROCESSING RATE $\ldots \ldots \ldots \ldots \ldots \ldots \ldots$

7.6 ADDITIONAL HIGH-LEVEL WASTE FEEDS $\ldots \ldots \ldots \ldots \ldots \ldots \ldots$

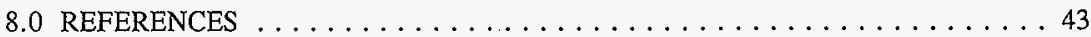


HNF-SD-TWR-AGA-003

Revision 0

\section{APPENDIXES}

A LIFE-CYCLE COST ESTIMATES FOR THE HIGH-LEVEL WASTE PRETREATMENT PROCESSING ALTERNATIVES $\ldots \ldots \ldots \ldots \ldots$ A-1

B SENSITIVITY STUDY RESULTS $\ldots \ldots \ldots \ldots \ldots \ldots \ldots$ B-1

C MATERIAL BALANCE TABLES $\ldots \ldots \ldots \ldots \ldots \ldots \ldots$ C 1

D HANFORD TANK WASTE OPERATION SIMULATOR RESULTS . . . . D - 1

E SCHEDULES OF WASTE TRANSFERS FOR THE PHASE I HIGHLEVEL WASTE PRETREATMENT PROCESSING ALTERNATIVES ... E-1

F PRELIMINARY EVALUATION OF PRETREATMENT ALTERNATIVES FOR TANKS 241-AY-101, 241-C-101, 241-C-102, 241-C-103, 241-C-104,

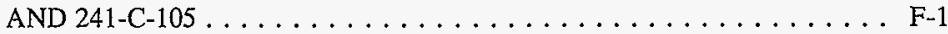

G PHASE I HIGH-LEVEL WASTE PRETREATMENT AND FEED STAGING IMPLEMENTATION SUMMARY $\ldots \ldots \ldots \ldots \ldots \ldots$ G-1

\section{LIST OF FIGURES}

2-1. Waste Movements During Phase I High-Level Waste Pretreatment and Feed Staging . . . . . . . . . . . . . . 4

3-1. Life-cycle Cost for Phase I Pretreatment Processing Alternatives (Based on BNFL, Inc., "Target-Unit-Prices") . . . . . . . . . . . . . . . . . . . . 12

3-2. Life-cycle Cost for Phase I Pretreatment Processing Alternatives (Based on LMAES

"Target-Unit-Prices") . . . . . . . . . . . . . . . . . . . 13 


\section{HNF-SD-TWR-AGA-003}

Revision 0

\section{LIST OF TABLES}

3-1. Influence of the Pretreatment Process Selection on the Pretreatment Equipment Selection $\ldots \ldots \ldots \ldots \ldots \ldots \ldots$

3-2. Phase I High-Level Waste Pretreatment Processing Alternatives $\ldots \ldots \ldots \ldots \ldots 6$

3-3. High-Level Waste Pretreatment Alternatives Decision Criteria Matrix: Contractual Considerations . . . . . . . . . . . . . . . . . .

3-4. High-Level Waste Pretreatment Alternatives Decision Criteria Matrix: Cost Minimization Considerations . . . . . . . . . . . . . . . 8

3-5. High-Level Waste Pretreatment Alternatives Decision Criteria Matrix: Other

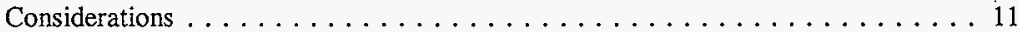

3-6. Minimum Life-cycle Cost Alternative(s) for BNFL, Inc. $\ldots \ldots \ldots \ldots \ldots$

3-7. Minimum Life-cycle Cost Alternative(s) for LMAES . . . . . . . . . . . . 17

6-1. Total Estimated Quantity ${ }^{a}$ of Waste Envelope D Feed Available from 241-AZ-101,

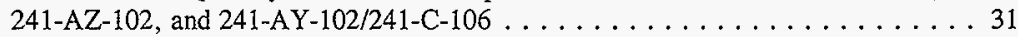

6-2. Phase I High-Level Waste Envelope D Comparison

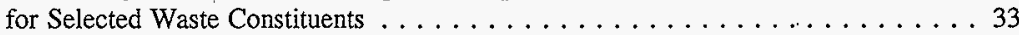

6-3. Phase I High-Level Waste Envelope D Comparison for Selected Waste Constituents.

6-4. High-Level Waste Feed Availability Assessment (Based on Sludge Washing) . . . . . 35

6-5. High-Level Waste Feed Availability Assessment

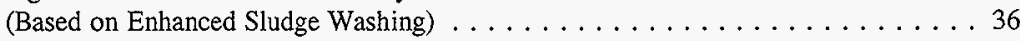

6-6. Estimated Low-Activity Waste Feed Quantities Resulting from High-Level Waste Pretreatment 
HNF-SD-TWR-AGA-003

Revision 0

\section{LIST OF TERMS}

$\begin{array}{ll}\text { AGA } & \text { Alternative generation and analysis } \\ \text { DOE } & \text { U.S. Department of Energy } \\ \text { DST } & \text { Double-shell tank } \\ \text { ESP } & \text { Environmental Simulation Program } \\ \text { FY } & \text { Fiscal year } \\ \text { HLW } & \text { High-level waste } \\ \text { HTWOS } & \text { Hanford Tank Waste Operation Simulator } \\ \text { ICR } & \text { Immobilization Cost Ratio } \\ \text { IPT } & \text { Integrated process/product team } \\ \text { LAW } & \text { Low-activity waste } \\ \text { PHMC } & \text { Project Hanford Management Contractor } \\ \text { RL } & \text { U.S. Department of Energy-Richland Operations Office } \\ \text { ROM } & \text { Rough order of magnitude } \\ \text { SST } & \text { Single-shell tank } \\ \text { TWRS } & \text { Tank Waste Remediation System } \\ \text { TWRSO\&UP } & \text { Tank Waste Remediation System Operation and Utilization Plan }\end{array}$


HNF-SD-TWR-AGA-003

Revision 0

This page intentionally left blank. 
HNF-SD-TWR-AGA-003

Revision 0

\section{ALTERNATIVES GENERATION AND ANALYSIS FOR THE PHASE I HIGH-LEVEL WASTE PRETREATMENT PROCESS SELECTION}

\subsection{OBJECTIVE}

This alternatives generation and analysis (AGA) was performed to address the question: Which pretreatment process, sludge washing or enhanced sludge washing, should be applied to each of the candidate high-level waste (HLW) sludge feeds from underground storage tanks, 241-AZ-101, 241-AZ-102, and 241-AY-102 (containing 241-C-106 sludge), to prepare them for delivery to the Private Contractor during Phase I? Once the pretreatment process is selected for each of these tanks, the next revision of this analysis will address the question: What is the design basis for the facilities (equipment) required to pretreat the HLW feeds prior to delivery to the Phase I Private Contractor?

\subsection{BACKGROUND AND SCOPE}

The U. S. Department of Energy, Richland Operations Office (DOE-RL), is pursuing a new business strategy for remediation of the Hanford Site tank waste. This strategy, commonly called Privatization, involves hiring private contractors to perform specific functions on a pay-for-product basis. The Private Contractors can recover the resources they have invested only upon delivery of acceptable waste treatment services. During Phase I of Privatization, the technical, operational, regulatory, and financial viability of the Privatization concept will be demonstrated by processing a portion of the waste in the double-shell tank (DST) system. The Phase I Privatization mission will include supernatant pretreatment, lowactivity waste (LAW) immobilization, and optionally, HLW immobilization. Phase II of Privatization will be the full-scale retrieval and processing of the remaining tank waste into forms suitable for final disposal.

The Phase IA Privatization contracts (DOE 1996a and DOE 1996b) establish requirements for the HLW feeds to be delivered to the Private Contractor during Phase I. These requirements apply to the quantity, composition and physical properties (i.e., Waste Envelope D), and schedule for the delivery of the HLW feeds. The feed specifications apply to both the U.S. Department of Energy (DOE) and the Private Contractor: the DOE must deliver feeds which satisfy the feed specifications, and the Private Contractor must accept feeds which satisfy the feed specifications. In purchasing waste treatment services, this contracting approach establishes both feed and product specifications, and allows the DOE to clearly specify and measure the performance of the Private Contractor. Therefore, the first objective for HLW pretreatment (i.e., sludge washing) is to satisfy the DOE's contractual commitments for Phase I operations, as discussed above. 
$\therefore$... The second objective for HLW pretreatment is cost minimization. Enhanced sludge washing (versus sludge washing) has the potential to reduce costs during Phase I operations (e.g, immobilized HLW disposal costs) and during the Phase I optional extension (e.g., payment for HLW immobilization services per the "Fixed-Unit-Prices" for extended order quantities). However, enhanced sludge washing will increase costs prior to Phase I operations (e.g., capital and operating costs for in-tank processing) and during the Phase I optional extension or Phase II (e.g., LAW immobilization costs). These economic trade-offs are evaluated for the alternative pretreatment processes.

The sludges contained in underground storage tanks 241-AZ-101, 241-AZ-102, and 241-AY-102 (containing 100 percent of the sludge from 241-C-106) are designated as the primary sources of Waste Envelope D feed. The pretreatment process selection will provide input to define the Disposal Program baseline to be updated in Revision 1 of the Tank Waste Remediation System Operation and Utilization Plan (TWRSO\&UP) (Kirkbride et al. 1997). The results of this evaluation will also be used to define the requirements to facilitate the pretreatment equipment selection, which will be addressed in the next revision of this document.

\subsection{PURPOSE AND NEED}

An evaluation of the Phase I HLW pretreatment process alternatives is necessary to reduce the risk of non-compliance with the Tank Waste Remediation System (TWRS) Privatization contract requirements for Phase I, and to provide the optimum processing strategy that minimizes cost. This evaluation also provides technical input during the establishment of the Phase IB contract (due May 1998) and interface requirements (due January 1998), and may also be used during the evaluation of Private Contractor proposals. Lastly, this document identifies the open issues, uncertainties, and potential risks pertinent to the selection of the Phase I HLW pretreatment process and to accomplishing the TWRS Phase I Privatization mission. 


\subsection{PHASE I HIGH-LEVEL WASTE FEED PREPARATION SUMMARY}

To prepare the HLW feeds for immobilization in Phase I, the sludges in the three DSTs will be separated into HLW and LAW fractions. This separations process (i.e., pretreatment) will take place in the source tanks, using a process known as sludge washing. In general, the purpose of sludge washing is to minimize the amount of material for HLW immobilization by dissolving and removing primarily non-radioactive chemicals from the HLW feeds.

Enhanced sludge washing (sometimes referred to as caustic leaching or caustic washing) represents the current baseline method for pretreating the Hanford Site tank waste. It is performed by adding a $50 \mathrm{wt} \%$ caustic (sodium hydroxide) solution to the waste, and mixing, to achieve a $1.0 \mathrm{M}$ to $3.0 \mathrm{M}$ hydroxide concentration in the supernatant. By chemical reaction, the addition of the caustic solution is expected to dissolve a significant amount of the precipitated aluminum compounds (e.g., Gibbsite), as well as other non-radioactive chemicals. The dissolution process is further improved by heating (Sterner et al. 1996). Elevation of the sludge temperature can be achieved during mixer pump operations and control of the ventilation system (Sathyanarayana 1994), or by steam injection.

Once the caustic-soluble compounds have dissolved, they are separated from the insoluble precipitates (i.e., from the HLW fraction) by gravity settling of the solid particles, and decantation of the liquid to another DST. (The decanted liquid can be potentially evaporated and then stored in another DST, eventually to be staged to the LAW treatment facility). The source DST containing the HLW fraction is successively refilled with a dilute caustic solution $(0.1 \mathrm{M}$ sodium hydroxide and $0.01 \mathrm{M}$ sodium nitrite), followed by mixing, settling, and decantation, in order to separate additional amounts of the dissolved chemicals.

Sludge washing without caustic leaching (sometimes referred to as dilute caustic washing or water washing) represents the minimum pretreatment for the HLW sludges. It is performed by decantation of the existing supernatant, followed by successively refilling the tank with dilute caustic, mixing, gravity settling, and decanting. Its purpose is primarily to dissolve and remove the water-soluble components (mainly sodium salts) from the sludge. Sludge washing typically utilizes a much smaller DST volume than enhanced sludge washing, because the process requires fewer wash repetitions, and, because the decanted liquid is less concentrated in sodium hydroxide, the solutions can be evaporated to a higher degree.

Figure 2-1 illustrates the waste transfers and processing operations required to prepare the three HLW feeds for delivery to the Phase I Private Contractor. The HLW feed preparation process involves several transfers of waste and wash solution within the DST system, prior to delivering the sludge to the Private Contractor. Step-by-step process descriptions and tables of the contents and compositions of each of the streams depicted in Figure 2-1 are presented in Appendix C. Schedules for each of the pretreatment processing steps are provided in Appendix E. 
HNF-SD-TWR-AGA-003

Revision 0

Figure 2-1. Waste Movements During Phase I High-Level Waste Pretreatment and Feed Staging.

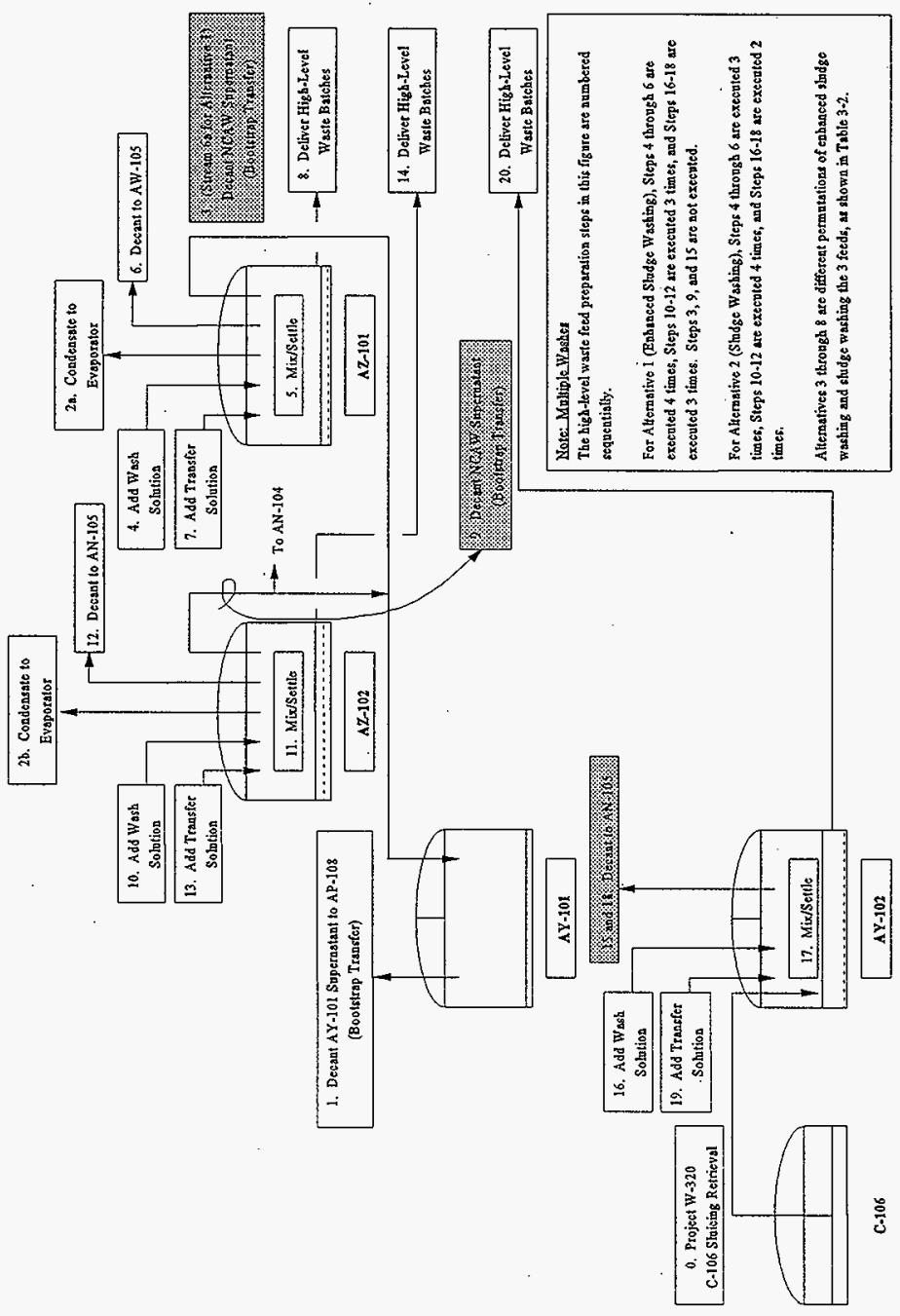




\subsection{DECISION ANALYSIS SUMMARY}

This section summarizes the pretreatment processing alternatives considered in this evaluation, and provides a comparison of the alternatives based on their performance against the decision criteria. Because the processing requirements need to be known before the processing equipment can be selected, the pretreatment equipment evaluation will be completed separately, in the next revision of this document.

\subsection{ALTERNATIVES GENERATION}

Based on the processing summaries in Section 2.0, the HLW pretreatment equipment will perform the following functions:

- Chemical Addition

- Waste Heating

- Waste Mixing

- Supernatant Decant/Transfer

- Waste Sampling.

The equipment selected to perform each of these functions is referred to as a system (e.g., the chemical addition system). Table 3-1 shows the influence of the pretreatment process selection on the pretreatment equipment selection.

Table 3-1. Influence of the Pretreatment Process Selection on the

Pretreatment Equipment Selection.

\begin{tabular}{|l|l|l|}
\hline $\begin{array}{l}\text { Components of the Phase I High- } \\
\text { Level Waste Pretreatment System }\end{array}$ & $\begin{array}{c}\text { Influence of the Process Selection } \\
\text { on the Equipment Selection }\end{array}$ & \multicolumn{1}{|c|}{ Justification } \\
\hline Chemical Addition System & Minimal Influence & $\begin{array}{l}\text { Estimates of capital and operating } \\
\text { costs and variations in costs for the } \\
\text { different equipment options are } \\
\text { negligible with respect to the overall } \\
\text { life-cycle costs for high-level waste } \\
\text { pretreatment, feed staging, } \\
\text { immobilization, and disposal. }\end{array}$ \\
\hline Waste Heating System & Minimal Influence & $\begin{array}{l}\text { The equipment functions are the } \\
\text { same for all pretreatment processing } \\
\text { alternatives. }\end{array}$ \\
\hline Waste Mixing System & No Influence & No Influence \\
\hline Supernatant Decant/Transfer System & No Influence & \\
\hline Waste Sampling System &
\end{tabular}

${ }^{a}$ Two 300-hp mixer pumps have already been installed into 241-AZ-101. 
As shown in Table 3-1, the cost variations between the alternatives are negligible with respect to the overall life-cycle costs. (Cost estimates for these systems are included in Appendix A). Therefore, to simplify this analysis and account for the equipment options associated with these systems, conservative rough-order-of-magnitude (ROM) estimates of capital and operating costs for chemical addition and waste heating are factored into the economic evaluation. (Non-economic performance measures for these systems, such as maintainability and operability, are also not expected to have a significant impact on the pretreatment process selection). The next revision of this document will address the equipment options for all five of the above systems.

\subsubsection{High-Level Waste Pretreatment Processing Alternatives}

Eight combinations of sludge washing and enhanced sludge washing the three Phase I HLW feeds were generated and then analyzed from an overall cost and benefit perspective. The eight alternatives are shown in Table 3-2.

Table 3-2. Phase I High-Level Waste Pretreatment Processing Alternatives.

\begin{tabular}{|c|c|c|c|c|c|c|c|c|}
\hline & \multicolumn{8}{|c|}{ Alternatives } \\
\hline & 1 & 2 & 3 & 4 & 5 & 6 & 7 & 8 \\
\hline 241-AZ-101 & (x) & SW & BSIV & SW & $8.811 \%$ & 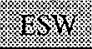 & SW & SW \\
\hline 241-AZ-102 & $1.581 \%$ & SW & $18 \mathrm{sil}$ & . 6511 & SW & SW & 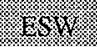 & SW \\
\hline $241-A Y-102^{a}$ & 1.50 & SW & SW & . 51 & ISI & SW & SW & $=18511$ \\
\hline
\end{tabular}

a241-AY-102 is assumed to contain 100 percent of the sludge from single-shell tank 241-C-106.

ESW $=$ Indicates that the HLW feed is enhanced sludge washed

HLW $=$ High-level waste

$\mathrm{SW}=$ Indicates that the HLW feed is sludge washed.

The analysis results are presented in tabular form (Tables 3-3, 3-4, and 3-5) and illustrated in Figures 3-1 and 3-2. The performance of each alternative above against the decision criteria is summarized. The decision criteria are categorized in terms of contractual considerations (Table 3-3), cost minimization considerations (Table 3-4), and other considerations (Table 3-5). Figures 3-1 and 3-2 compare the life-cycle costs for each . alternative. Since the "Fixed-Unit-Prices" for processing the extended order quantities of HLW and LAW feed are not yet known, a range is assumed. The "Immobilization Cost Ratio" (ICR), ranges between 0.0 and 1.0, and is defined as the ratio between the assumed "FixedUnit-Prices" for the extended order quantities of HLW and LAW feed to the "Target-UnitPrices" for the minimum order quantities of HLW and LAW feed. 
Table 3-3. High-Level Waste Pretreatment Alternatives Decision Criteria Matrix: Contractual Considerations.

\begin{tabular}{|c|c|c|c|c|c|c|c|c|}
\hline \multirow{2}{*}{$\begin{array}{l}\text { Decision Criteria - } \\
\text { Contractual } \\
\text { Considerations }\end{array}$} & \multicolumn{8}{|c|}{ Alternatives } \\
\hline & 1 & 2 & 3 & 4 & 5 & 6 & 7 & 8 \\
\hline \multirow{3}{*}{$\begin{array}{l}\text { Ability to Satisty the } \\
\text { Minimum Order } \\
\text { Quantity of HLW } \\
\text { Feed }^{\text {a.b }}\end{array}$} & ND & $\mathrm{ND}$ & ND & ND & ND & ND & ND & ND \\
\hline & \multicolumn{8}{|c|}{ The percent of the minimum order quantity ${ }^{a}$ is estimated at: } \\
\hline & 105 to $136 \%$ & 142 to $184 \%$ & 120 to $153 \%$ & 116 to $148 \%$ & 117 to $156 \%$ & 131 to $172 \%$ & 131 to $165 \%$ & 128 to $168 \%$ \\
\hline $\begin{array}{l}\text { Ability to Satisfy the } \\
\text { HLW Feed } \\
\text { Specifications (Waste } \\
\text { Envelope D) }\end{array}$ & Lowest & Highest & Medium & Medium & Medium & Medium & Medium & Medium \\
\hline $\begin{array}{l}\text { Ability to Provide a } \\
\text { Continuous High-Level } \\
\text { Waste Feed }\end{array}$ & ND & ND & ND & ND & ND & ND & ND & ND \\
\hline $\begin{array}{l}\text { Ability to satisfy the } \\
\text { Low-Activity Waste } \\
\text { Envelope B } \\
\text { Specitications }\end{array}$ & Lowest & Highest & Medium & Medium & Medium & Medium & Medium & Medium \\
\hline
\end{tabular}

ND: Non-Discriminating

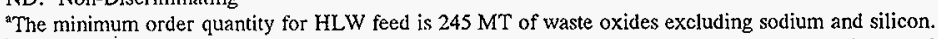

${ }^{6}$ Based on the availability of additional HLW feeds (241-AY-101, C-101, C-102, C-103, C-104, and C-105) identified in Kirkbride et al. (1997), and the assumption that the Phase IB contracts will include these additional feeds for the extended order quantities, this is a non-discriminating factor between the alternatives.

'Based on the operating scenarios developed using the Hanford Tank Waste Operation Simulator (HTWOS) provided in Appendix D, this is a nondiscriminating factor between the alternatives. 
Table 3-4. High-Level Waste Pretreatment Alternatives Decision Criteria Matrix: Cost Minimization Considerations. (Sheet 1 of 3)

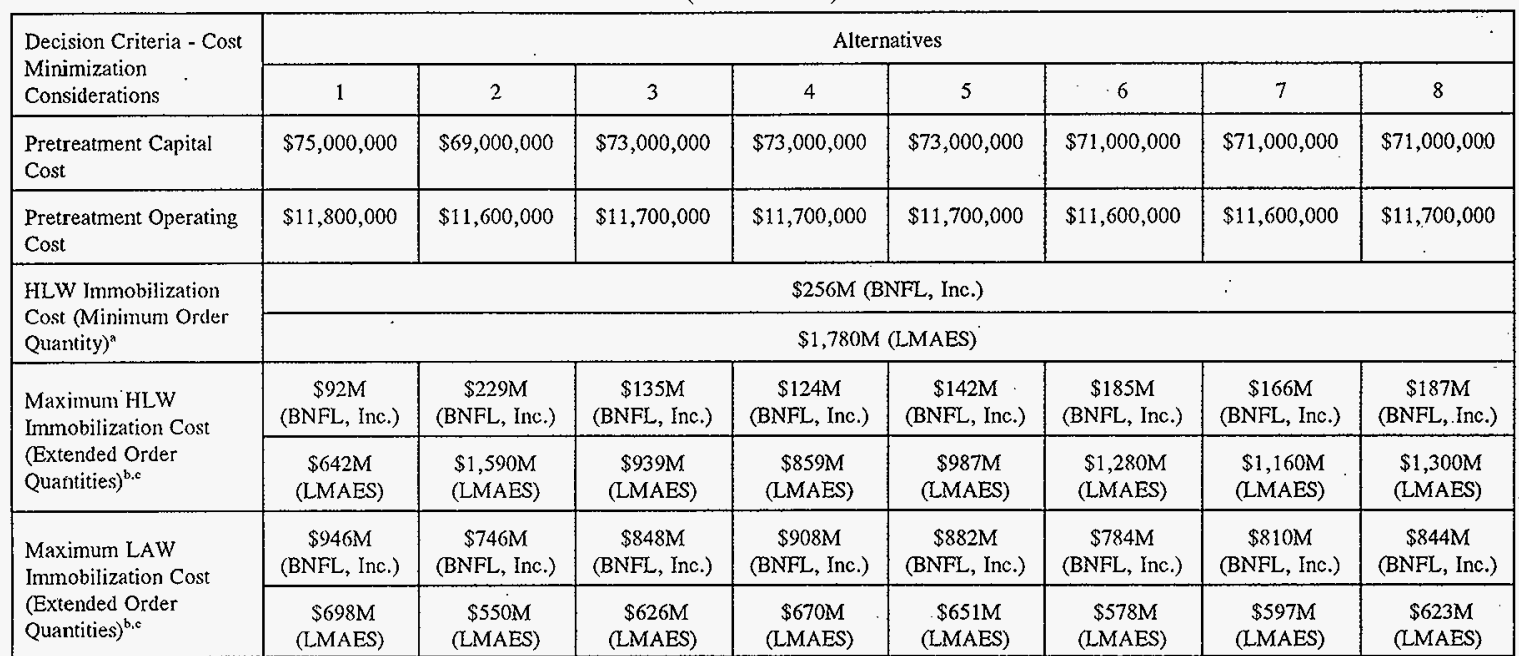

"The "Fixed-Unit-Prices" for HLW and LAW immobilization services for the minimum order quantities (to be established during Phase IA) are assumed to be equal to the "Target-Unit-Prices" (for the minimum order quantities) in the Phase IA contracts (DOE 1996a and b).

"The "Fixed-Unit-Prices" for HLW and LAW immobilization services for quantities of feed in excess of the minimum order quantities (i.e., for extended order quantities) have not been established. The maximum "Fixed-Unit-Prices" for the extended order quantities are assumed to be equal to the "Target-UnitPrices" for the minimum order quantities. Because of this uncertainty, this analysis assumes that the "Fixed-Unit-Prices" for the extended orler quantities will be between 0 and 100 percent of the "Target-Unit-Prices" for the minimum order quantities.

"The ratio of the "Fixed-Unit-Prices" for HLW feeds to the "Fixed-Unit-Prices" for LAW feeds is assumed to be the same for the minimum and extended order quantities (for each Private Contractor). The ratio of assumed "Fixed-Unit-Prices" for the extended order quantities (of HLW and LAW feed) to the "Fixed-Unit-Prices" for the minimum order quantities (of HLW and LAW feed) is referred to as the "Immobilization Cost Ratio." Therefore, the maximum HLW and LAW immobilization costs in this table correspond to an "Immobilization Cost Ratio" of 1.0. 
Table 3-4. High-Level Waste Pretreatment Alternatives Decision Criteria Matrix: Cost Minimization Considerations.

(Sheet 2 of 3)

\begin{tabular}{|c|c|c|c|c|c|c|c|c|}
\hline \multirow{2}{*}{$\begin{array}{l}\text { Decision Criteria - Cost } \\
\text { Minimization } \\
\text { Considerations } \\
\text { (continued) }\end{array}$} & \multicolumn{8}{|c|}{ Alternatives } \\
\hline & 1 & 2 & 3 & 4 & 5 & 6 & 7 & 8 \\
\hline \multirow{3}{*}{$\begin{array}{l}\text { HLW Canister Interim } \\
\text { Storage Cost }\end{array}$} & $\$ 30,200,000$ & $\$ 40,900,000$ & $\$ 33,900,000$ & $\$ 32,900,000$ & $\$ 34,500,000$ & $\$ 38,200,000$ & $\$ 36,600,000$ & $\$ 37,200,000$ \\
\hline & \multicolumn{8}{|c|}{ Number of $1.08-\mathrm{m}^{3}$ canisters, based on the minimum HLW product loading: } \\
\hline & 464 & 629 & 521 & 506 & 531 & 587 & 563 & 571 \\
\hline $\begin{array}{l}\text { HLW Canister Disposal } \\
\text { Cost }^{\mathbf{a}}\end{array}$ & $\$ 196,000,000$ & $\$ 265,000,000$ & $\$ 220,000,000$ & $\$ 213,000,000$ & $\$ 224,000,000$ & $\$ 248,000,000$ & $\$ 237,000,000$ & $\$ 241,000,000$ \\
\hline \multirow{3}{*}{ LAW Disposal Cost ${ }^{b}$} & $\$ 11,000,000$ & $\$ 8,660,000$ & $\$ 9,850,000$ & $\$ 10,500,000$ & $\$ 10,200,000$ & $\$ 9,110,000$ & $\$ 9,400,000$ & $\$ 9,800,000$ \\
\hline & \multicolumn{8}{|c|}{ Volume $\left(\mathrm{m}^{3}\right)$ of LAW product resulting from HLW pretreatment (includes the initial decants from 241-AZ-101 and 241-AZ-102): } \\
\hline & 2890 & 2280 & 2590 & 2770 & 2690 & 2400 & 2480 & 2580 \\
\hline
\end{tabular}

${ }^{2}$ Based on a $\$ 422,000$ incremental HLW disposal cost per $1.08-\mathrm{m}^{3}$ canister (DOE 1996c) and a $\$ 65,000$ incremental interim storage cost per canister (Calmus 1996).

based on a LAW disposal cost of $\$ 3800 / \mathrm{m}^{3}$, based on FM-20 cost estimates.

'Based on a package volume of $73.1 \mathrm{~cm}^{3} /$ gmole sodium (Assumption 7.23 from Kirkbride et al. [1997]). 
Table 3-4. High-Level Waste Pretreatment Alternatives Decision Criteria Matrix: Cost Minimization Considerations. (Sheet 3 of 3)

\begin{tabular}{|c|c|c|c|c|c|c|c|c|}
\hline \multirow{2}{*}{$\begin{array}{l}\text { Decision Criteria - Cost } \\
\text { Minimization } \\
\text { Considerations } \\
\text { (continued) }\end{array}$} & \multicolumn{8}{|c|}{ Alternatives } \\
\hline & 1 & 2 & 3 & 4 & 5 & 6 & 7 & 8 \\
\hline \multirow{5}{*}{$\begin{array}{l}\text { Cost for DST Storage of } \\
\text { Decanted Liquids } \\
\text { Resulting from HLW } \\
\text { Pretreatment }\end{array}$} & ND & ND & ND & ND & ND & $\mathrm{ND}$ & ND & $\mathrm{ND}$ \\
\hline & \multicolumn{8}{|c|}{ Volume of Decanted Liquids Before Evaporation (excludes the initial decants from 241-AZ-101 and 241-AZ-102): } \\
\hline & $4,010 \mathrm{kgal}$ & $1,810 \mathrm{kgal}$ & $3,370 \mathrm{kgal}$ & $3,320 \mathrm{kgal}$ & $3,150 \mathrm{kgal}$ & $2,500 \mathrm{kgal}$ & $2,670 \mathrm{kgal}$ & $2,450 \mathrm{kgal}$ \\
\hline & \multicolumn{8}{|c|}{ Volume of Decanted Liquids After Evaporation (excludes the initial decants from 241-AZ-101 and 241-AZ-102): } \\
\hline & $1,490 \mathrm{kgal}$ & $304 \mathrm{kgal}$ & $1,180 \mathrm{kgal}$ & $957 \mathrm{kgal}$ & $1,140 \mathrm{kgal}$ & $833 \mathrm{kgal}$ & $648 \mathrm{kgal}$ & $613 \mathrm{kgal}$ \\
\hline Life-cycle Cost & $\begin{array}{l}\text { Variable (See } \\
\text { Figures } 3-2 \\
\text { and } 3-3 \text { ). }\end{array}$ & $\begin{array}{l}\text { Variable (See } \\
\text { Figures } 3-2 \\
\text { and } 3-3 \text { ). }\end{array}$ & $\begin{array}{c}\text { Variable (See } \\
\text { Figures 3-2 } \\
\text { and 3-3). }\end{array}$ & $\begin{array}{c}\text { Variable (See } \\
\text { Figures 3-2 } \\
\text { and } 3-3 \text { ). }\end{array}$ & $\begin{array}{c}\text { Variable (See } \\
\text { Figures 3-2 } \\
\text { and } 3-3 \text { ). }\end{array}$ & $\begin{array}{c}\text { Variable (See } \\
\text { Figures } 3-2 \\
\text { and } 3-3 \text { ). }\end{array}$ & $\begin{array}{c}\text { Variable (See } \\
\text { Figures 3-2 } \\
\text { and 3-3). }\end{array}$ & $\begin{array}{c}\text { Variable (See } \\
\text { Figures 3-2 } \\
\text { and 3-3). }\end{array}$ \\
\hline
\end{tabular}

ND: Based on the operating scenarios developed using the Hanford Tank Waste Operation Simulator (HTWOS) provided in Appendix D, this is a nondiscriminating factor between the alternatives.

"The operating and capital cost impacts associared with these volumes of decanted liquids have not been assessed. 
Table 3-5. High-Level Waste Pretreatment Alternatives Decision Criteria Matrix: Other Considerations.

\begin{tabular}{|c|c|c|c|c|c|c|c|c|}
\hline \multirow{2}{*}{$\begin{array}{l}\text { Decision Criteria - } \\
\text { Other }\end{array}$} & \multicolumn{8}{|c|}{ Alternatives } \\
\hline & 1 & 2 & 3 & 4 & 5 & 6 & 7 & 8 \\
\hline $\begin{array}{l}\text { Technology } \\
\text { Demonstration }\end{array}$ & $\begin{array}{l}\text { Lowest } \\
\text { See Note A } \\
\text { below. }\end{array}$ & $\begin{array}{l}\text { Lowest } \\
\text { See Note B } \\
\text { below. }\end{array}$ & $\begin{array}{l}\text { Lowest } \\
\text { See Note A } \\
\text { below. }\end{array}$ & $\begin{array}{l}\text { Lowest } \\
\text { See Note A } \\
\text { below. }\end{array}$ & $\begin{array}{l}\text { Lowest } \\
\text { See Note A } \\
\text { below. }\end{array}$ & Medium & $\begin{array}{l}\text { Highest } \\
\text { See Note C } \\
\text { below. }\end{array}$ & Medium \\
\hline Safety & ND & ND & ND & ND & ND & ND & ND & ND \\
\hline
\end{tabular}

ND: This is a non-discriminating factor between the alternatives.

Note A: If enhanced sludge washing technology needs to be demonstrated, it does not need to be conducted for more than one HLW feed. The cost implications (a separate decision criterion) will dictate whether other tanks should be enhanced sludge washed.

Note B: Alternative 2 does not demonstrate enhanced sludge washing.

Note C: Alternative 7 is the lowest cost alternative in which the sludge in only one tank is enhanced sludge washed (see Figures 3-1 and 3-2): 
HNF-SD-TWR-AGA-003

Revision 0

Figure 3-1. Life-cycle Cost for Phase I Pretreatment Processing Alternatives (Based on BNFL, Inc., "Target-Unit-Prices").

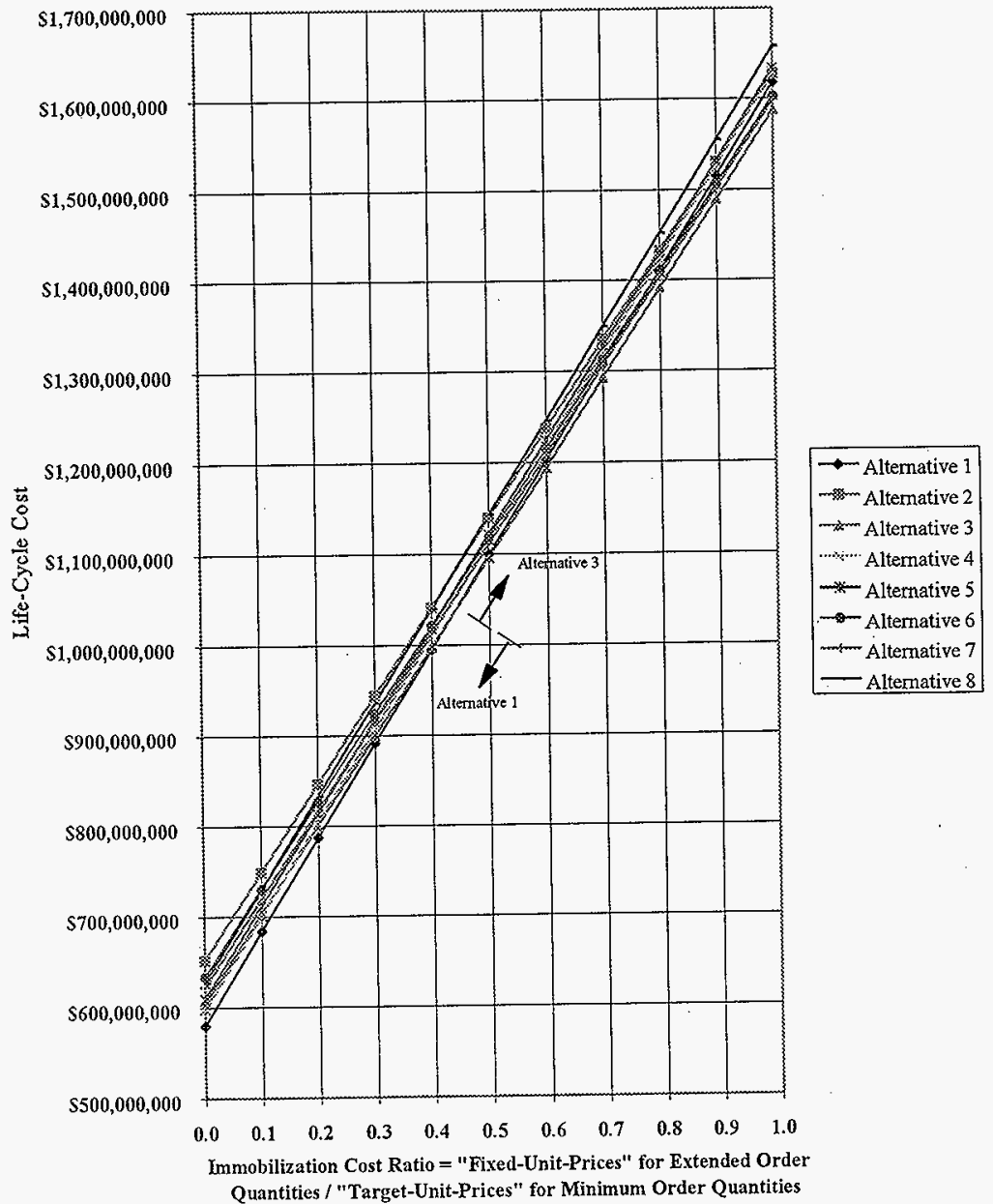


HNF-SD-TWR-AGA-003

Revision 0

Figure 3-2. Life-cycle.Cost for Phase I Pretreatment Processing Alternatives (Based on LMAES "Target-Unit-Prices").

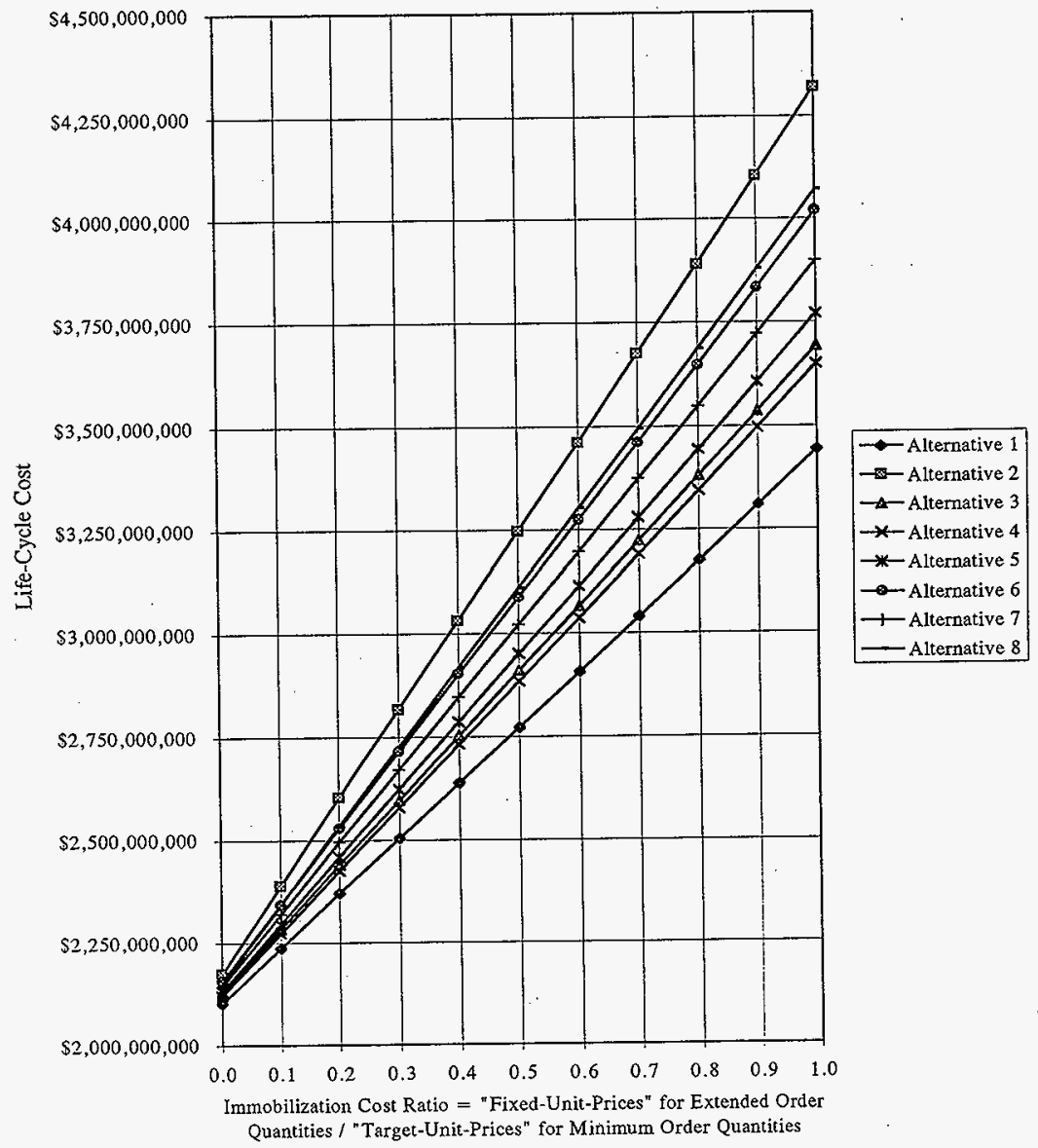


HNF-SD-TWR-AGA-003

Revision 0

\subsubsection{High-Level Waste Pretreatment Equipment Alternatives}

The sludge washing equipment alternatives to perform the recommended processing in 241-AZ-101, 241-AZ-102, and 241-AY-102 will be addressed in the next revision of this analysis. The assumptions are provided in Sections 5.2.1 and 5.2.2.

\subsection{CONCLUSIONS}

This section summarizes the primary findings from this analysis to assist the decision board regarding the proposed alternatives. These comparisons will provide the basis for the board's recommendation of the preferred Phase I HLW pretreatment processing alternative. Unresolved contractual issues and technical uncertainties regarding the HLW immobilization process (discussed in Section 7.0) exist and will influence this decision. Therefore, any decision made prior to the final Phase IB Privatization contract awards in May 1998, must be conditional. When the Phase IB contract terms are finalized in fiscal year (FY) 1998, a final decision can be made.

\subsubsection{Contractual Considerations}

To minimize the risk of non-compliance with the requirements and specifications established in the Phase IA Privatization contracts, Alternative 2 should be selected. This alternative is to sludge wash (dilute caustic wash) all three HLW feeds. Following pretreatment, this alternative will require the least amount of chemical adjustment to satisfy the Waste Envelope D concentration limits (refer to Section 6.1.2). As summarized in Table 3-3, between 142 and 184 percent of the minimum. order quantity ${ }^{l}$ of HLW feed can be provided to the Private Contractor, although this decision criteria is non-discriminating based on the availability of additional HLW feeds (Kirkbride et al. 1997). Alternative 2 also poses the least risk of affecting the compositions of the 241-AZ-101 and 241-AZ-102 supernatants designated for LAW Envelope B feed.

From a contractual tisk perspective, Alternative 1- (enhanced sludge wash all HLW feeds) performs the worst. The estimated compositions of the pretreated HLW feeds will be further outside of the Waste Envelope D composition limits (as shown in Tables 6-2 and 6-3), and will require the highest amount of chemical adjustment to satisfy the Waste Envelope D concentration limits. Between 105 and 136 percent of the minimum order quantity of HLW feed can be provided to the Private Contractor. Lastly, although the compositions of the initial decant liquids from 241-AZ-101 and 241-AZ-102 are not estimated to fall outside of the Waste Envelope $B$ feed specifications, this alternative increases the contractual risk because it alters the supernatant compositions.

${ }^{1}$ The minimum order quantity of HLW feed is $245 \mathrm{MT}$ of waste oxides excluding sodium and silicon. 


\section{HNF-SD-TWR-AGA-003 \\ Revision 0}

The HTWOS model (described in Kirkbride et al. 1997) has been used to verify that all of the eight pretreatment processing alternatives can be performed within the existing DST space (see Appendix D). The minimum processing rate for HLW immobilization can be supported with zero idle time. Therefore, the ability to provide a continuous HLW feed is not a discriminating factor between the alternatives. (Increased immobilization throughput rates will be addressed in the next revision of Kirkbride et al. [1997]).

This study assumes that the HLW and LAW feed specifications are fixed. From this perspective, Alternative 2 represents the minimum contractual risk alternative. However, this conciusion is subject to change if allowances for flexibilities in pretreatment processing are provided in the final feed specifications (in the Phase IB contracts). For example, the HLW feed specifications may be broadened to include both enhanced sludge washed and sludge washed feeds, so they include the estimated analyte concentrations in Tables 6-2 and 6-3. Adding this flexibility would allow the Project Hanford Management Contractor (PHMC) to perform other pretreatment alternatives without increasing contractual risk.

\subsubsection{Cost Minimization Considerations}

The life-cycle cost estimates do not include the capital and operating cost impacts associated with the handling of decanted liquids resulting from HLW pretreatment. However, they can be considered negligible in this evaluation, because the HTWOS model has been used to verify the existence of sufficient DST space and enough schedule flexibility to accomplish any of the eight pretreatment processing alternatives with zero immobilization facility idle time (see Appendix D).

The costs for storage of the decanted liquids could be substantial enough to influence the pretreatment process selection, since they are strongly dependent upon the availability of DST space. Since this evaluation shows that sufficient tank space is available to perform any of the pretreatment processing alternatives, the cost differences are negligible. However, if changes in program assumptions result in the conclusion that there isn't enough DST space, the costs may be equivalent to any of the following:

1. The cost to provide additional storage capacity

2. The cost penalty incurred by delaying HLW feed delivery (which is a contractual risk consideration, as well)

3. The cost of interfering with other waste transfers, such as LAW feed staging, which may also have cost penalties if delays are incurred.

Although Alternative 2 is the minimum contractual risk alternative (as discussed in Section 3.2.1), it may not be the alternative which will minimize life-cycle cost. However, the life-cycle costs depend on information which is not currently available. For BNFL, Inc., the minimum cost alternative cannot be determined, due to the uncertainties in the "Fixed- 


\section{Revision 0}

Unit-Prices" and the immobilized HLW product volumes. However, as shown in Figures 3-1 and B-1, the cost differential between the alternatives (for BNFL, Inc.) is negligible with respect to overall life-cycle cost. Table 3-6 summarizes the results of the cost evaluation for BNFL, Inc.

Table 3-6: Minimum Life-cycle Cost Alternative(s) for BNFL, Inc.

\begin{tabular}{|c|c|c|}
\hline Minimum cost alternative & $\begin{array}{c}\mathrm{a} \\
\text { Immobilization cost ratio } \\
\text { (ICR) }\end{array}$ & $\begin{array}{c}\text { Assumed immobilized high- } \\
\text { level waste product volume } \\
\text { reduction (enhanced sludge } \\
\text { washing versus sludge } \\
\text { washing) }\end{array}$ \\
\hline 1 & $<0.45$ & $\begin{array}{c}\text { Proportional to the reduction } \\
\text { in the HLW feed quantity } \\
\text { (see Figure 3-1) }\end{array}$ \\
\hline 3 & $>0.45$ & None (see Figure B-1) \\
\hline 2 & $<0.3$ & \\
\hline 6 & $>0.3$ & \\
\hline
\end{tabular}

${ }^{a}$ All of the alternatives are within $\$ 50$ million of each other, which is negligible with respect to the overall life-cycle cost.

"The "Immobilization Cost Ratio" (ICR), which ranges between 0.0 and 1.0 , is defined as the ratio between the assumed "Fixed-Unit-Prices" for the extended order quantities of HLW and LAW feed to the "Target-Unit-Prices" for the minimum order quantities of HLW and LAW feed.

${ }^{c}$ Assumes 25 wt $\%$ non-volatile oxides excluding sodium and silicon in the immobilized high-level waste product.

${ }^{d}$ Assumes the immobilized high-level waste product volumes are the same for both enhanced sludge washing and sludge washing.

For BNFL, Inc, the Base Case comparison in Figure 3-1 and Sensitivity Case \#1 in Figure B-1 indicate that the minimum cost alternative will depend on information on the costs for processing the extended order quantities and the immobilized HLW product volumes.

For LMAES, Figures 3-2 and B-2 indicate that Alternative 2 consistently results in a higher life-cycle cost, regardless of the differences in HLW disposal costs or the "Immobilization Cost Ratio." In the LMAES case, Alternative 1 is the preferred alternative. However, to implement a different alternative will mean to proceed with increased contractual risk, unless the Privatization contract specifications can be modified to accommodate the range of concentrations as shown in Section 6.1.2, Table 6-3. (Note: The lowest life-cycle cost. estimate for LMAES is higher than the highest life-cycle cost estimate for BNFL, Inc.). Table 3-7 summarizes the cost minimization results for LMAES. 
Table 3-7. Minimum Life-cycle Cost Alternative(s) for LMAES.

\begin{tabular}{|c|c|c|}
\hline Minimum cost alternative & $\begin{array}{c}\text { Immobilization Cost Ratio } \\
\text { (ICR) }^{\mathrm{a}}\end{array}$ & $\begin{array}{c}\text { Assumed immobilized high- } \\
\text { level waste product volume } \\
\text { reduction (enhanced sludge } \\
\text { washing versus sludge } \\
\text { washing) }\end{array}$ \\
\hline 1 & 0.0 to 1.0 & $\begin{array}{c}\text { Proportional to the reduction } \\
\text { in the HLW feed quantity } \\
\text { (see Figure 3-2) }\end{array}$ \\
\hline 1 & 0.0 to 1.0 & \multirow{2}{*}{ None (see Figure B-2) ${ }^{\mathrm{c}}$} \\
\hline 1 & 0.0 to 1.0 & \\
\hline 1 & 0.0 to 1.0 & \\
\hline
\end{tabular}

"The "Immobilization Cost Ratio" (ICR), which ranges between 0.0 and 1.0, is defined as the ratio between the assumed "Fixed-Unit-Prices" for the extended order quantities of HLW and LAW feed to the "Target-Unit-Prices" for the minimum order quantities of HLW and LAW feed.

${ }^{\mathrm{b}}$ Assumes $25 \mathrm{wt} \%$ non-volatile oxides excluding sodium and silicon in the immobilized high-level waste product.

'Assumes the immobilized high-level waste product volumes are the same for both enhanced sludge washing and sludge washing.

Table 3-7 indicates that the minimum cost alternative is Alternative 1, regardless of the "Immobilization Cost Ratio" or the basis for estimating the immobilized HLW product volume. Therefore, if the LMAES "Target-Unit-Prices" are used as the basis for evaluating cost minimization, Alternative 1 can be selected.

The conclusion of this analysis is that due to the uncertainties in which Private Contractor will perform HLW immobilization, the uncertainties in the "Fixed-Unit-Prices" for the minimum and maximum order quantities of HLW and LAW feed, and the uncertainties in the method for estimating the immobilized HLW product volumes (see Appendix B), the minimum cost alternative cannot be determined. Once this information is available, the cost evaluation should be revised.

\subsubsection{Other Considerations}

Ignoring the contractual and cost minimization criteria, Alternative 6 for BNFL, Inc. or Alternative 7 for LMAES performs the best at accomplishing the goals of a technology demonstration. The Base Case life-cycle cost evaluation shows that Alternative 7 has the minimum cost, while the Sensitivity Cases in Appendix B show that the minimum cost alternative depends on the Private Contractor. 
A demonstration of the caustic leaching process is only necessary in one tank (i.e., Alternatives 6,7 , or 8 can be chosen). Therefore, depending on the Private Contractor who performs HLW immobilization, Alternative 6 or Alternative 7 will be the lowest cost alternative in which the sludge in only one tank is enhanced sludge washed.

The pretreatment processing alternatives cannot be differentiated on the basis of safety. Therefore, the safety decision criterion is disregarded.

\subsection{DESIGN REQUIREMENTS RECOMMENDATIONS}

Alternatives 1, 2, 3,6, and 7 are preferred over Alternatives 4, 5, and 8, due to cost minimization, the ability to satisfy Waste Envelopes B and D, and technology demonstration considerations. These conclusions and the criteria for "down" selection are described in Section 3.2. However, a final recommendation for the specific alternative must be deferred until the following information is available:

- . The "Fixed-Unit-Prices" for processing the minimum and maximum order quantities of HLW and LAW feed (for each Private Contractor).

- The range and expected value of the immobilized HLW product loading based on an enhancèd sludge washed feed and a sludge washed feed (for each Private Contractor).

This information needs to be requested from the Private Contractors by the conclusion of Phase IA. The deferral of this decision until mid-FY 1998 will not adversely impact the pretreatment equipment selection, due to the relatively small impact of the equipment capital costs on the life-cycle cost for pretreatment (see Section 3.1).

\subsection{DESIGN REQUIREMENTS DECISION}

This document does not contain a decision on the Phase I HLW pretreatment system. A decision board will be convened in early FY 1998 to make a preliminary decision. The decision will be revised after the Phase IB contracts are awarded (May 1998). 
HNF-SD-TWR-AGA-003

Revision 0

\subsection{PROBLEM STATEMENT:}

To support the Phase I HLW immobilization option, this AGA report evaluates the factors affecting the selection of the preferred pretreatment processing alternative (sludge washing or enhanced sludge washing) for the HLW sludge in DSTs, 241-AZ-101, 241-AZ-102, and 241-AY-102 (containing 241-C-106 sludge). The pretreatment process selection will influence contractual compliance/risk, cost minimization, and other criteria, such as technology demonstration and safety. The next revision of this AGA will update this evaluation, based on information from the Private Contractors' Phase IA deliverables (e.g., technical reports) and the Phase IB contract(s). A final decision will be made, regarding the pretreatment processing alternative, and the equipment required will also be determined to support this decision.

\subsection{SOURCE DOCUMENTS}

The following section discusses the primary source documents that provide input to the set of requirements and performance criteria established for the Phase I HLW pretreatment system under consideration in this AGA. Performance parameters (decision criteria) for cost minimization are not limited to those which are published in these documents, and the full set of decision criteria are described in Section 5.0.

\subsubsection{Tank Waste Remediation System Phase I Privatization Contracts DE-RP06-96RL13308 (BNFL, Inc.) and DE-RP06-96RL13309 (LMAES)}

Privatization Phase I consists of two parts: Part A and Part B. For Part A, the "Phase IA" contracts (DOE 1996a and DOE 1996b) establish a set of specifications for the HLW immobilization feed. These contracts specify a compositional range (or "envelope") of chemical and radioactive constituents of the HLW feed to be delivered to the Private Contractor. The contracts also define the ranges for selected physical properties of the HLW feed. This range is referred to as Specification 8 , or Waste Envelope D.

In accordance with the "Indefinite Quantity" clause, ${ }^{1}$ the DOE shall order waste treatment (immobilization) services for at least the minimum order quantity of HLW feed, and the Private Contractor shall provide waste treatment services for up to the maximum order quantity. Upon delivery of an immobilized HLW product satisfying all contract requirements, the Private Contractor will be paid for waste treatment services based on the "Fixed-UnitPrices" established during Phase IA.

${ }^{1}$ TWRS Privatization contracts, Section I (DOE 1996a and DOE 1996b). 
Part $A$ is a 20 -month development period to establish the technical, operational, regulatory, business, and financial elements required by privatized facilities. Based on the Phase IA contract requirements and specifications, the Private Contractors will complete deliverables which describe specific technical aspects and plans for Phase I operations (Part B).

If the funds are available, the DOE may authorize one or more of the Private Contractors who perform successfully in Part A to perform Part B work. During Part B, waste treatment services will be provided to the DOE at the "Fixed-Unit-Prices" agreed to in Part A. Wastes will be processed during a 5- to 9-year period during Phase IB, starting on June 1, 2002.

The specific requirements for Part B will be stipulated in the Phase IB contracts, which are yet to be established. The Phase IB contract awards are scheduled for May 1998. All Phase IA deliverables must be completed by January 1998, however the Phase IB contract negotiations, including some critical decision-making, may extend until the contract award date.

This evaluation is based on the Phase IA contracts. The conclusions reached may not be valid once the Phase IB contracts are awarded. Therefore, the HLW pretreatment process selection will need to be revised in FY 1998 to minimize the risk of non-compliance with the Phase IB contract terms.

\subsubsection{Tank Waste Remediation System Operation and Utilization Plan}

The Phase I High-Level Waste Pretreatment and Feed Staging Plan is provided in the TWRSO\&UP (Kirkbride et al. 1997). The plan describes the baseline strategy for preparing the HLW feeds for delivery to the Private Contractor. It also describes the base case constraints, requirements, and assumptions used in the evaluation. The base case assumes sludge washing (dilute caustic washing) the 241-AZ-101, 241-AZ-102, and 241-AY-102/241-C-106 feeds (i.e., Alternative 2 of this AGA). The HLW feed preparation strategy is currently being updated as part of this AGA to support Revision 1 of Kirkbride et al. (1997).

The estimated chemical and radionuclide inventories for each tank and the caustic leach factors (i.e., the fraction of solid phase chemicals dissolved by the addition of $50 \mathrm{wt} \%$ caustic) are provided in Kirkbride et al. (1997). The processing assumptions for the base case are given, while the alternative cases considered for this evaluation are presented in Appendixes $C$, $D$, and $E$ of this AGA. 


\subsubsection{Computer Simulation of the Leaching and Washing of Waste in Tanks 241-C-106, 241-AY-102, 241-AZ-101, and 241-AZ-102}

A chemical process simulation computer program called the Environmental Simulation Program (ESP) was used for purposes of evaluating the compositions and quantities of the wastes in 241-AZ-101, 241-AZ-102, and 241-AY-102/241-C-106 after pretreatment processing (MacLean 1997). Both sludge washing and enhanced sludge washing were modeled. The ESP simulations were used to baiance valances and predict the species and phases present in each tank waste. The optimum processing conditions were evaluated, and the amounts of caustic (sodium hydroxide) solution and water required were predicted.

The results of the ESP simulations were compared with the results of related laboratory and miniplant leaching and washing experiments. These ESP calculations were supplied as input to this AGA, to evaluate the effect of pretreatment on the immobilization facility feed specifications, and to provide an immobilization feed stream to use to predict the volume of immobilized HLW product. 
HNF-SD-TWR-AGA-003

Revision 0

This page intentionally left blank. 


\section{HNF-SD-TWR-AGA-003 \\ Revision 0}

\subsection{DECISION CRITERIA}

This section identifies the performance measures used to differentiate between the eight alternatives. It is important to note that the performance measures represent a mixture of quantitative and qualitative factors. Some of the performance measures, such as cost, can be quantified for each of the alternatives and compared. Other performance measures, such as a technology demonstration, cannot be quantified and are therefore much more dependent on the judgement of experienced engineers and are therefore compared in narrative form.

This study focuses on the more tangible and immediately visible performance measures, such as contractual compliance and cost minimization. Although, it should be noted that some of the less tangible performance measures, can also carry hidden cost and performance penalties. For example, the absence of a technology demonstration of caustic leaching in Phase I to provide information of its effectiveness in full-scale processing may result in schedule delays during larger-scale production in Phase II. However, obtaining this information by demonstrating caustic leaching may be accompanied by a near-term cost penalty, as discussed in Section 5.2 .

\subsection{CONTRACTUAL CONSWERATIONS}

Because the Phase IB Privatization contracts will impose requirements on both the DOE and the Private Contractor, a major concern for selecting the HLW pretreatment process is contract compliance. The pretreatment process will affect the characteristics of the HLW feeds, for which specific requirements have been established. ${ }^{1}$ The primary contract terms associated with the HLW feeds are the order quantity, the feed specifications, the feed delivery schedule, and the implications on LAW Envelope B. These contractual considerations are discussed below.

\subsubsection{Ability to Satisfy the Minimum Order Quantity of High-Level Waste Feed}

A minimum of $245 \mathrm{MT}$ of waste oxides excluding sodium and silicon must be delivered to the HLW Private Contractor between June 1, 2002 and June 1, 2007. The HLW feed will be delivered in several batches via pipeline to the Private Contractor's facility, according to a schedule yet to be established for Phase IB. HLW feed sources in addition to the base case tanks have been identified in Kirkbride et al. (1997), and an operational scenario for delivering

'As discussed in Section 4.1.1, the contract terms for waste treatment services have not yet been established for Part B. The current Phase IA contracts contain specifications to be used for the Part A deliverables (e.g., technical reports and "Fixed-Unit-Prices"). As an enabling assumption in this analysis, it is assumed that the Phase IB contract terms will be the same as or similar to the Phase IA contract terms, with minor negotiated modifications. 
this feed is being developed for Revision 1 of Kirkbride et al. (1997). Enhanced sludge : washing will reduce the total quantity of Waste Envelope D feed. This decision criterion (quantity of feed) has a related cost influence (see Section 5.2.3).

\subsubsection{Ability to Satisfy the High-Level Waste Feed Specifications}

The purpose of performing pretreatment on the HLW sludges is to alter the composition of the feed to immobilization. Enhanced sludge washing has a much greater effect on the HLW immobilization feed composition than sludge washing. Therefore, each alternative will have a different effect on the ability to comply with the HLW feed specifications (i.e., with Waste Envelope D). This decision criterion has a related cost influence (see Section 5.2.5).

As stated previously, the Phase IA contracts impose requirements on both the DOE and the Private Contractor. However, it should be noted that the Private Contractor is not required to limit the HLW immobilization facility to processing Waste Envelope D feeds only. But, the DOE is limited to delivering Waste Envelope D feeds during Phase I. Therefore, these specifications are subject to change for Phase IB, and flexibilities in the immobilization facility design (a subject of the Private Contractors' technical reports) may have an influence on the finalized HLW feed specifications for Phase IB.

\subsubsection{Ability to Provide a Continuous High-Level Waste Feed}

Enhanced sludge washing imposes added equipment and operational demands on the DST system. Without imposing significant additional costs (e.g., constructing new tanks), these demands may have an influence on the ability to provide a continuous HLW feed to the Private Contractor. Also, because sludge washing involves several waste transfers within the DST system, as shown in Figure 2-1, the alternatives may also have impacts on LAW feed staging activities and the ability to meet the LAW feed delivery schedule requirements.

The feed delivery schedule is a function of the HLW immobilization processing rate. A minimum processing rate of $60 \mathrm{MT}$ of waste oxides excluding sodium and silicon per year is specified in the Phase IA contracts, although the actual rate will not be known until FY 1998. This decision criterion has a related cost influence (see Section 5.2.7).

\subsubsection{Ability to Satisfy the Low-Activity Waste Envelope B Specifications}

As described in Section 2.0, enhanced sludge washing will involve the addition of $50 \mathrm{wt} \%$ caustic solution, prior to the first liquid decant. Thus, the dissolution process will alter the composition of the initial decant liquid, which for 241-AZ-101 and 241-AZ-102, is currently designated as LAW Envelope B feed. Therefore, the pretreatment processing alternatives must consider the risk of altering the composition of these LAW feeds to the extent that they cannot satisfy the Waste Envelope B feed specifications. Tanks 241-AZ-101 and 
241-AZ-102 are currently the only sources of LAW supernatant which will satisfy. Waste. Envelope B.

\subsection{COST MINIMIZATION CONSIDERATIONS}

In addition to satisfying the Privatization contract specifications for the HLW feeds, there are also cost minimization considerations for HLW pretreatment processing. The selection of the pretreatment process will affect life-cycle cost in the following areas:

- Pretreatment Capital Cost

- Pretreatment Operating Cost

- High-Level Waste Immobilization Cost (Minimum and Extended Order Quantities)

- Low-Activity Waste Immobilization Cost (Extended Order Quantities)

- High-Level Waste Canister Interim Storage and Disposal Cost

- Low-Activity Waste Disposal Cost

- Cost for DST Storage of Decanted Liquids Resulting from HLW Pretreatment.

These cost considerations must be balanced against the contractual considerations discussed in Section 5.1. In some cases, they may provide a reasonable basis for modifying the contract requirements and specifications.

\subsubsection{Pretreatment Capital Cost}

The equipment necessary to implement enhanced sludge washing includes a caustic addition system and an in-tank heating system. Since the pretreatment processing equipment selection will be performed separately, as discussed in Section 3.1, a conservative (high) estimate of these systems has been made. The volume of caustic solution and amount of heat required are estimated to be low enough (MacLean 1997), so that significant capital upgrades are not required to perform these functions. Therefore, this cost is not expected to bias the decision (based on life-cycle cost).

\subsubsection{Pretreatment Operating Cost}

The pretreatment operating cost for each alternative will vary, depending on the demand for sodium hydroxide $(\$ 250 / \mathrm{MT})$ and steam $(\$ 5 / \mathrm{MT}$ at $15 \mathrm{Mpa})$. Pretreatment also indirectly influences the operating cost in the rest of the DST system, due to transfers of liquids out of the tank(s) being pretreated, but this indirect cost is not expected to have a significant impact on the pretreatment process selection. 


\section{5:2.3 High-Level Waste Immobilization Cost (Minimum and Extended Order Quantities)}

During Privatization Phase IA, the "Fixed-Unit-Prices" will be established for processing Waste Envelope $\mathrm{D}$ feeds between the minimum and maximum feed order quantities (i.e., the extended order quantities). Depending on the final contract negotiations and available funding, there may be an incentive to minimize the quantity of HLW feed to be processed during the Phase I extension.

Minimizing the amount of HLW feed by enhanced sludge washing does not come without consequence, however. It may increase costs in other areas, such as for processing additional sodium (resulting from additional sodium hydroxide needs) in the LAW fraction (Section 5.2.4). This decision criterion has a related contractual compliance impact (Section 5.1.1).

Because the "Fixed-Unit-Prices" for the extended order quantities are not known, this evaluation is based on different ratios of assumed "Fixed-Unit-Prices" for the extended order quantities to the "Target-Unit-Prices" for the minimum order quantities stated in the Phase IA contracts. The ratio between the unit price for HLW immobilization and the unit price for LAW immobilization is assumed constant for both the minimum and extended order quantities of feed.

Each candidate HLW Private Contractor has proposed a different "Target-Unit-Price" for the HLW and LAW feeds. It is assumed that the "Target-Unit-Prices" for the minimum order quantities of HLW and LAW feed will not differ significantly from the "Fixed-Unit-Prices" for the minimum order quantities, which are yet to be established. Significant differences between the "Fixed-" and "Target-" prices may invalidate the conclusions of this analysis.

\subsubsection{Low-Activity Waste Immobilization Cost (Extended Order Quantities)}

Because of the greater caustic solution (sodium hydroxide) demand, enhanced sludge washing versus sludge washing increases the amount of sodium in the DST system. Because the costs for LAW treatment services are based on sodium, the added sodium will have an effect on the LAW immobilization costs.

The sodium resulting from HLW pretreatment processing will either be processed during the Phase I extension or during Phase II of Privatization. The "Fixed-Unit-Prices" for LAW treatment services have not been established for either for the Phase I extension or Phase II. Therefore, as discussed in Section 5.2.3, these costs will be based on a ratio to the "TargetUnit-Price" for LAW treatment services in the Phase IA contracts. 


\subsubsection{High-Level Waste Canister Interim Storage and Disposal Cost}

The cost for geologic disposal of the immobilized HLW product can be reduced by decreasing the total volume of immobilized HLW product. Aside from simply reducing the amount of feed, this reduction can be accomplished by varying the extent of pretreatment, by reducing the amount of specific waste constituents which influence the waste oxide loading. Selective removal processes, such as caustic leaching, may be beneficial in this regard. To summarize, pretreatment processing by enhanced sludge washing affects the HLW product volume by the following:

1. Reducing the concentrations of specific waste constituents which limit the waste oxide loading in the immobilized HLW product (e.g., aluminum).

2. Reducing the mass of non-radioactive components from the sludge (i.e, from the insoluble solids and interstitial liquid) to reduce the quantity of HLW feed to be immobilized.

Achieving the first objective by enhanced sludge washing (if for example, aluminum oxide limits the waste oxide loading), increases the Private Contractor's ability to achieve higher waste oxide loadings, thereby reducing the HLW product volume(s). A waste minimization performance incentive for the Private Contractor to.maximize the waste oxide loading to the highest achievable value will be established to encourage the Private Contractor to do so. Waste minimization performance incentives will be established in Phase IA, as stated in contract clause H.6 of the Phase IA contracts (DOE 1996a and DOE 1996b).

Achieving the second objective by enhanced sludge washing allows the DOE to reduce the cost for HLW immobilization, but a reduction in the HLW product volume still depends on the waste oxide loading actually achieved by the Private Contractor. For example, the Private Contractor may achieve a high waste oxide loading with simple sludge washing, but might be limited by the iron content of the HLW feed. If the HLW feeds are enhanced sludge washed, the Private Contractor will have to replace the aluminum removed with additional glass formers to bring the concentration of iron oxide down to acceptable levels in the product. In doing so, the Private Contractor decreases the waste oxide loading, but produces the same volume of HLW product.

Since the Private Contractor's immobilized HLW product formulation is not known, and since the waste minimization performance incentives have not been established, the volume of immobilized HLW is based on the minimum 25 wt \% waste oxide loading (excluding sodium. and silicon) for this evaluation. Sensitivity cases, based on no assumed reduction in immobilized HLW and on the glass property models from Lambert et al. (1996), are evaluated in Appendix A. 


\subsubsection{Low-Activity Waste Disposal Cost}

Similar to HLW disposal, LAW also has associated costs for onsite disposal. Consistent with Kirkbride et al. (1997), estimates of LAW product volumes are based on a LAW product package volume of $73.1 \mathrm{~cm}^{3} / \mathrm{gmole}$ sodium.

\subsubsection{Cost for Double-Shell Tank Storage of Decanted Liquids Resulting From High-Level Waste Pretreatment}

The transfers of wash solutions and supernatants (illustrated in Figure 2-1) throughout the DST system will have implications on tank space usage. The eight alternatives are evaluated based on the ability to perform the necessary processing steps on schedule and within the available DST space. In this evaluation, the HTWOS model is used to determine if and how much additional DST space is required, and what schedule conflicts or delays are incurred if the waste transfers for a specific alternative are completed within the existing DST space. Appendix D presents the results of the HTWOS evaluation.

If no additional DST space is required, it is assumed that this cost consideration has a minimal influence on life-cycle cost. The cost differences between the alternatives would be mostly operating cost for performing transfers within the DST system.

If additional DST space is required, the capital cost to provide the additional DST capacity calculated by the HTWOS model, will be the basis for quantifying this decision criterion. As an alternative, the schedule delays due to the failure to deliver waste feed(s) on time, as calculated by the HTWOS model, will be equated to an assumed penalty for "idle facilities." Phase IA contract clause H.30 states that the Private Contractor(s) must submit their proposals for a daily idle facilities payment rate prior to Phase IB operations. These costs will be revised when this information becomes available for the next revision of this study.

\subsection{OTHER CONSIDERATIONS}

As stated previously, the following decision criteria are not quantifiable, and their consideration is mostly dependent upon the judgement of experienced engineers. Their value is primarily based on trade-offs with the other decision criteria.

\subsubsection{Technology Demonstration}

All eight alternatives demonstrate the effectiveness of sludge washing technology and sludge mobilization. The sludge mobilization information is needed to predict sludge retrieval performance and to test the effectiveness of the mixing system for contacting wash solutions with the solids. 


\section{Revision 0}

With the exception of Alternative 2, the alternatives all demonstrate the effects of caustic leaching (enhanced sludge washing). The remaining 7 alternatives will also demonstrate the operation of the chemical addition and waste heating systems. The number of tanks in which enhanced sludge washing needs to be demonstrated is subject to engineering judgement. As an

- enabling assumption, enhanced sludge washing needs to be demonstrated in only one tank (i.e., Alternatives 6,7 , or 8 ).

\subsubsection{Safety}

To the extent practical and meaningful, alternatives should be compared on the bases of associated hazards and implications for onsite/offsite safety, worker safety, and mission and property protection. Topical areas for consideration include the following:

- Hazards

- Introduction/creation of hazards

- $\quad$ Ease of hazard prevention

- $\quad$ Ease of hazard mitigation

- Offsite/Onsite safety

- Hazard categorization

- Safety class

- Performance category, or seismic criteria

- Radiological risk acceptance criteria compliance

- Toxicological risk acceptance criteria compliance

- Process and industrial safety

- Health physics requirements

- Compliance with DOE 6430.1A and related industry standards

- Mission and Property Protection

- Potential for accident propagation and impacts to other facilities

- Potential impacts due to accidents initiated at other facilities

- Implications for recovery from accidents expected to occur during the lifetime of the mission. 
HNF-SD-TWR-AGA-003

Revision 0

This page intentionally left blank. 
HNF-SD-TWR-AGA-003

Revision 0

\subsection{ALTERNATIVES EVALUATION AND ANALYSIS}

This section presents the detailed evaluations for each of the alternatives against the decision criteria discussed in Section 5.0. Sensitivity studies regarding technical uncertainties, such as the waste oxide loading, which may also affect the outcome of the decision-making process, are covered in Appendix B.

\subsection{CONTRACTUAL CONSIDERATIONS}

The following analysis is based on the "Base Case" HLW pretreatment and feed staging information from Kirkbride et al. (1997), and additional cases provided in Appendixes C, D, and $\mathrm{E}$. Chemical compositions and inventories are derived from "Best-Basis Inventory" estimates (Kupfer et al. 1996), and ESP modeling (MacLean 1997) of sludge dissolution chemistry.

\subsubsection{Ability to Satisfy the Minimum Order Quantity of High-Level Waste Feed}

Table 6-1 summarizes the estimated quantities of HLW feed available, by pretreatment processing alternative.

Table 6-1. Total Estimated Quantity ${ }^{a}$ of Waste Envelope D Feed Available from 241-AZ-101, 241-AZ-102, and 241-AY-102/241-C-106.

\begin{tabular}{|l|c|c|c|c|c|c|c|c|}
\cline { 2 - 9 } \multicolumn{1}{c|}{} & \multicolumn{7}{c|}{ Alternatives } \\
\cline { 2 - 8 } \multicolumn{1}{c|}{} & 1 & 2 & 3 & 4 & 5 & 6 & 7 & 8 \\
\hline $\begin{array}{l}\text { Maximum (100\%) } \\
\text { Retrieval Efficiency }\end{array}$ & 334 & 452 & 374 & 363 & 381 & 422 & 404 & 411 \\
\hline $\begin{array}{l}\text { Minimum Retrieval } \\
\text { Efficiency }\end{array}$ & 258 & 348 & 293 & 285 & 287 & 322 & 320 & 314 \\
\hline
\end{tabular}

${ }^{\mathrm{a}} \mathrm{MT}$ of waste oxides excluding sodium and silicon.

${ }^{\mathrm{b}}$ Minimum Retrieval Efficiency $=90,60$, and 85 percent retrieval for 241-AZ-101, 241-AZ-102, and 241-AY-102/241-241-C-106, respectively (derived from Grams [1995]).

The quantity of feed available from the primary Waste Envelope D source tanks will depend strongly on the ability of the DST retrieval systems to remove the pretreated solids from each tank. The results of mixer pump operations testing following the completion of Project W-151, "Tank 241-AZ-101 Waste Retrieval System," in FY 1998, will provide some useful 
information on the degree of solids mobilization. However, a sludge retrieval demonstration is not currently planned. The critical information needs resulting from the implications of this technical uncertainty are discussed in Appendix G.

If the quantities retrieved are equal to the minimum percent mobilization from Grams (1995), Alternative 1 comes very close to the minimum order quantity, or 245 MT of waste oxides excluding sodium and silicon. This alternative may require delivery of one or more of the additional HLW feeds identified in the TWRSO\&UP (i.e., 241-AY-101, 241-C-101, 241-C-102, 241-C-103, 241-C-104, and/or 241-C-105). This decision criterion is considered non-discriminating because it assumed that the Phase IB HLW feed specifications for the extended order quantities will include these feeds. Information on the additional feeds is included in Appendix F.

\subsubsection{Ability to Satisfy the High-Level Waste Feed Specifications}

Tables 6-2 and 6-3 present comparisons of the estimated HLW feed compositions from 241-AZ-101, 241-AZ-102, and 241-AY-102/241-C-106 with Waste Envelope D and the Expanded Design Basis composition limits for Waste Envelope D. Table 6-2 presents the results of sludge washing, and Table 6-3 presents the results of enhanced sludge washing. 
Table 6-2. Phase I High-Level Waste Envelope D Comparison for Selected Waste Constituents. (Based on $31 \mathrm{~g} / \mathrm{L}$ Non-Volatile Waste Oxides and Sludge Washing)

\begin{tabular}{|c|c|c|c|c|c|}
\hline \multirow{2}{*}{ Component } & \multirow{2}{*}{$\begin{array}{l}\text { Minimum } \\
\text { concentration } \\
(\mathrm{g} / \mathrm{L})\end{array}$} & \multicolumn{3}{|c|}{$\begin{array}{l}\text { Estimated high-level waste feed concentrations } \\
\text { after sludge washing (g/L) }\end{array}$} & \multirow{2}{*}{$\begin{array}{l}\text { Maximum } \\
\text { concentration } \\
(g / L)\end{array}$} \\
\hline & & 241-AZ-101 & $241-\mathrm{AZ}-102$ & $\begin{array}{c}241-\mathrm{AY}-102 / \\
241-\mathrm{C}-106 \\
\end{array}$ & \\
\hline Aluminum & $1.30(0.33)$ & 2 & (2, & 4.25 & $4.30(5.30)$ \\
\hline Bismuth & 0.00 & 0.009 & 0.006 & 0.0008 & 0.86 \\
\hline Chlorine & 0.00 & 0.005 & 0.0002 & 0.01 & 0.10 \\
\hline Chromium & 0.00 & 0.10 & 0.12 & 0.11 & $0.21(0.42)$ \\
\hline Fluorine & 0.00 & 0.18 & 0.19 & 0.003 & 1.10 \\
\hline Iron & $2.60(1.70)$ & 6.16 & 8.17 & 6.23 & $8.90(13.00)$ \\
\hline Manganese & 0.00 & 1.37 & 0.18 & 0.30 & 2.00 \\
\hline Nickel & $0.05(0.00)$ & 0.36 & 0.55 & 0.09 & $0.73(1.00)$ \\
\hline Palladium & 0.00 & $\mathrm{NE}$ & NE & $\mathrm{NE}$ & 0.04 \\
\hline Phosphorus & 0.00 & 0.06 & 0.06 & 0.44 & 0.54 \\
\hline Potassium & 0.00 & $\mathrm{NE}$ & NE & 0.03 & 0.41 \\
\hline Rhodium & 0.00 & $\mathrm{NE}$ & $\mathrm{NE}$ & $\mathrm{NE}$ & 0.04 \\
\hline Ruthenium & 0.00 & $\mathrm{NE}$ & $\mathrm{NE}$ & $\mathrm{NE}$ & 0.11 \\
\hline Silicon & 0.00 & 0.36 & 0.58 & 2.14 & 5.80 \\
\hline Silver & 0.00 & NE & $\mathrm{NE}$ & 1026 & 0.17 \\
\hline Sodium & $2.30(1.00)$ & 3.45 & 165 & 4.22 & $6.00(9.20)$ \\
\hline Sulfur & 0.00 & 0.15 & 0.11 & 0.03 & 0.20 \\
\hline Titanium & 0.00 & $\mathrm{NE}$ & $\mathrm{NE}$ & $\mathrm{NE}$ & 0.40 \\
\hline Uranium & 0.00 & 0.64 & 1.61 & 0.04 & 4.20 \\
\hline Zirconium & 0.00 & 2.16 & 1.13 & 0.06 & 4.60 \\
\hline
\end{tabular}

DOE $=$ U.S. Department of Energy

HLW $=$ High-level waste

TWRS $=$ Tank Waste Remediation System

General Notes

- Values enclosed in parentheses are for the Expanded Design Basis for HLW Processing as defined in the Phase I TWRS Privatization contracts (DOE 1996a and DOE 1996b).

- Shaded rows indicate component concentrations which are close to or outside of the HLW feed specifications.

- Analytes not shown are within the HLW feed specifications and/or have no significant impact on the immobilized HLW product. 


\section{Revision 0}

Table 6-3. Phase I High-Level Waste Envelope D Comparison for Selected Waste Constituents.

(Based on $31 \mathrm{~g} / \mathrm{L}$ Non-Volatile Waste Oxides and Enhanced Sludge Washing)

\begin{tabular}{|c|c|c|c|c|c|}
\hline \multirow{2}{*}{ Component } & \multirow{2}{*}{$\begin{array}{l}\text { Minimum } \\
\text { concentration } \\
(\mathrm{g} / \mathrm{L})\end{array}$} & \multicolumn{3}{|c|}{$\begin{array}{l}\text { Estimated high-level waste feed concentrations } \\
\text { after enhanced sludge washing }(\mathrm{g} / \mathrm{L})\end{array}$} & \multirow{2}{*}{$\begin{array}{c}\text { Maximum } \\
\text { concentration } \\
(\mathrm{g} / \mathrm{L})\end{array}$} \\
\hline & & $241-A Z-101$ & 241-AZ-102 & $\begin{array}{c}24 l-\mathrm{AY}-102 / \\
241-\mathrm{C}-106 \\
\end{array}$ & \\
\hline Aluminum & $1.30(0.33)$ & 8.69 & (18) & (2) & $4.30(5.30)$ \\
\hline Bismuth & 0.00 & 0.01 & 0.008 & 0.0009 & 0.86 \\
\hline Chlorine & 0.00 & 0.003 & 0.0002 & 0.009 & 0.10 \\
\hline Chromium & 0.00 & 0.14 & 0.16 & 0.13 & $0.21(0.42)$ \\
\hline Fluorine & 0.00 & 0.24 & 0.02 & 0.002 & 1.10 \\
\hline Iron & $2.60(1.70)$ & 3.. & (1) & 7.50 & $8.90(13.00)$ \\
\hline Manganese & 0.00 & 26 & 0.25 & 0.36 & 2.00 \\
\hline Nickel & $0.05(0.00)$ & 0.54 & (x) & 0.11 & $0.73(1.00)$ \\
\hline Palladium & 0.00 & $\mathrm{NE}$ & $\mathrm{NE}$ & NE & 0.04 \\
\hline Phosphorus & 0.00 & 0.09 & 0.09 & 0.35 & 0.54 \\
\hline Potassium & 0.00 & NE & NE & 0.02 & 0.41 \\
\hline Rhodium & 0.00 & NE & NE & $\mathrm{NE}$ & 0.04 \\
\hline Ruthenium & 0.00 & $\mathrm{NE}$ & $\mathrm{NE}$ & $\mathrm{NE}$ & 0.11 \\
\hline Silicon & 0.00 & 0.44 & 0.72 & 2.50 & 5.80 \\
\hline Silver & 0.00 & $\mathrm{NE}$ & $\mathrm{NE}$ & 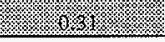 & 0.17 \\
\hline Sodium & $2.30(1.00)$ & 2.47 & 26 & 4.43 & $6.00(9.20)$ \\
\hline Sulfur & 0.00 & 0.09 & 0.09 & 0.02 & 0.20 \\
\hline Titanium & 0.00 & NE & $\mathrm{NE}$ & $\mathrm{NE}$ & 0.40 \\
\hline Uranium & 0.00 & 0.98 & 2.27 & 0.03 & 4.20 \\
\hline Zirconium & 0.00 & 3.27 & 1.60 & 0.07 & 4.60 \\
\hline
\end{tabular}

DOE $=$ U.S. Department of Energy

$\mathrm{HLW}=$ High-level waste

TWRS $=$ Tank Waste Remediation System

General Notes

- Values enclosed in parentheses are for the Expanded Design Basis for HLW Processing as defined in the Phase I TWRS Privatization contracts (DOE 1996a and DOE 1996b).

- Shaded rows indicate component concentrations which are close to or outside of the HLW feed specifications.

- Analytes not shown are within the HLW feed specifications and/or have no significant impact on the immobilized HLW product. 
The shaded cells in Tables 6-2 and 6-3 indicate components which are estimated to be at concentrations outside of the composition limits for Waste Envelope $D$ following pretreatment. To comply with the Waste Envelope D specifications will require slight chemical adjustment by dissolution with caustic solution and decantation (i.e., a "mini-" caustic wash) to reduce aluminum concentrations, and/or the addition of inert chemicals, such as sodium and silicon compounds, to dilute or "chemically shim" the concentrations of chemicals which are high and cannot be washed out of the feeds (i.e., silver, iron, manganese, and nickel). (The "chemical shimming" can be performed with glass-forming materials, so as not to increase the Private Contractor's immobilized HLW product volume.

This analysis indicates that enhanced sludge washing of any of the HLW feeds will increase the amount of chemical adjustment required to satisfy Waste Envelope D. Alternatively, it increases the amount of modifications to the HLW feed specifications necessary. Therefore, Alternative 2 has the highest ability to satisfy this decision criterion.

\subsubsection{Ability to Provide a Continuous High-Level Waste Feed}

Appendix D contains the waste transfer schedules for the two extremes in terms of the impact of the alternatives on DST space utilization (i.e., Aiternatives 1 and 2). The results, which are generated from the HTWOS model, indicate that for either case, the base case feed delivery schedule is not compromised for either alternative. Therefore, this decision criterion is not a discriminating factor for consideration in this evaluation. Tables 6-4 and 6-5 summarize the results of the HTWOS calculations.

Table 6-4. High-Level Waste Feed Availability Assessment (Based on Sludge Washing).

\begin{tabular}{|l|c|c|c|c|c|c|c|c|}
\cline { 2 - 7 } \multicolumn{1}{c|}{} & \multicolumn{8}{c|}{ Feed Availability Efficiency } \\
\cline { 2 - 7 } \multicolumn{1}{c|}{} & \multicolumn{8}{c|}{ Alternative } \\
\hline $\begin{array}{l}\text { High-Level Waste } \\
\text { Processing Rate }\end{array}$ & 1 & 2 & 3 & 4 & 5 & 6 & 7 & 8 \\
\hline Minimum $^{\mathrm{b}}$ & $100 \%$ & $100 \%$ & \multicolumn{6}{c|}{ Not Evaluated } \\
\hline
\end{tabular}

${ }^{2}$ Feed Availability Efficiency $=$ Percent of the time during Phase IB that high-level waste feed is available for immobilization

${ }^{\mathrm{b}} 60 \mathrm{MT}$ of waste oxides excluding sodium and silicon per year. 


\section{HNF-SD-TWR-AGA-003}

\section{Revision 0}

Table 6-5. High-Level Waste Feed Availability Assessment (Based on Enhanced Sludge Washing).

\begin{tabular}{|l|c|c|c|c|c|c|c|c|}
\cline { 2 - 7 } \multicolumn{1}{c|}{} & \multicolumn{8}{c|}{ Feed Availability Efficiency } \\
\cline { 2 - 7 } \multicolumn{1}{c|}{} & \multicolumn{9}{c|}{ Alternative } \\
\hline $\begin{array}{l}\text { High-Level Waste } \\
\text { Processing Rate }\end{array}$ & 1 & 2 & 3 & 4 & 5 & 6 & 7 & 8 \\
\hline Minimum $^{\mathrm{b}}$ & $100 \%$ & $100 \%$ & \multicolumn{6}{c|}{ Not Evaluated } \\
\hline
\end{tabular}

${ }^{a}$ Feed Availability Efficiency $=$ Percent of the time during Phase IB that high-level waste feed is available for immobilization

b60 MT of waste oxides excluding sodium and silicon per year.

\subsubsection{Ability to Satisfy the Low-Activity Waste Envelope B Specifications}

This analysis concludes that the compositions of supernatants initially decanted from 241-AZ-101 and 241-AZ-102 during enhanced sludge washing will be altered. However, they will not be altered to the extent that Waste Envelope $B$ cannot be satisfied. These conclusions will need to be verified by the Feed Process Control Group, which establishes configuration control on the tank contents.

\subsection{COST MINIMIZATION CONSIDERATIONS}

A summary evaluation of the pretreatment processing alternatives versus the cost minimization criteria is presented in Section 3.2.2. The details of the cost analysis are shown in Appendix A.

\subsection{OTHER CONSIDERATIONS}

The results are summarized in Section 3.2.3.

\subsection{SUPPLEMENTAL INFORMATION}

The quantities of LAW feed resulting from pretreatment operations are presented in Table 6-6. 
HNF-SD-TWR-AGA-003

Revision 0

Table 6-6. Estimated Low-Activity Waste Feed Quantities Resulting from High-Level Waste Pretreatment. ${ }^{2}$

\begin{tabular}{|l|c|c|c|c|c|c|c|c|}
\cline { 2 - 8 } \multicolumn{1}{c|}{} & \multicolumn{7}{c|}{ Alternatives } \\
\cline { 2 - 8 } \multicolumn{1}{c|}{} & 1 & 2 & 3 & 4 & 5 & 6 & 7 & 8 \\
\hline $\begin{array}{l}\text { Low-Activity } \\
\text { Waste Feed (MT } \\
\text { of Sodium) }\end{array}$ & 910 & 717 & 815 & 873 & 848 & 754 & 779 & 812 \\
\hline
\end{tabular}

ancludes the initial decants from 241-AZ-101 and 241-AZ-102

(i.e., the Low-Activity Waste Envelope B Feeds). 
HNF-SD-TWR-AGA-003

Revision 0

This page intentionally left blank. 


\section{HNF-SD-TWR-AGA-003 \\ Revision 0}

\subsection{OPEN ISSUES AND ACTIONS}

Open issues exist which may have an influence on this decision. The resolution of these issues will take place in studies which are yet to be completed, and at the conclusion of Privatization Phase IA, when Private Contractor design information will be available and Privatization contract requirements for Phase IB are finalized. It may be possible to resolve some of these issues in the interim through integrated process/product team (IPT) support. Other uncertainties and issues may remain until the conclusion of Phase IA, when the Phase IB contract specifications are established.

\section{1 "FIXED-UNIT-PRICES"-}

As shown in Figures 3-1 and 3-2, the pretreatment processing alternative which minimizes life-cycle cost is dependent upon the "Fixed-Unit-Prices" for the extended order quantities of HLW feed. The costs for processing the LAW resulting from HLW pretreatment processing may be incurred during either the Phase I extension or Phase II. In this analysis, it is assumed that the LAW unit costs will be the same as the "Fixed-Unit-Prices" agreed to for the LAW extended order quantities. The preferred alternative cannot be selected until the "Fixed-Unit-Prices" for processing the minimum and maximum order quantities of HLW and LAW feed are known.

Action: This AGA should be revised when the "Fixed-Unit-Prices" for HLW and LAW processing of the minimum and extended order quantities are finalized. These prices are expected to be finalized in May 1998.

\subsection{IMMOBILIZED HIGH-LEVEL WASTE PRODUCT VOLUME}

In this evaluation, for a given pretreatment alternative, the minimum immobilized HLW product loading (25 wt\% non-volatile oxides excluding sodium and silicon) is assumed. The sensitivity cases in Appendix B assume product loadings based on Lambert et al. (1996) and Hrma et al. (1994). However, the Private Contractor may propose an alternative method for estimating the product volume, based on the specific immobilization facility design. This uncertainty in the Private Contractor's ability to maximize the waste oxide loading will influence the estimates of life-cycle cost in this analysis, and the selection the preferred pretreatment process.

A reduction in the HLW product volume will translate into savings in production (to the Private Contractor), interim storage, and disposal costs (Manuel et al. 1996). Therefore, since neither the basis for predicting the HLW product volume nor the Private Contractor's incentive to maximize the HLW product loading are known, an exact comparison of the HLW disposal costs cannot be performed for the alternatives. Once the "Fixed-Unit-Prices" are known, this uncertainty may have an impact on the preferred alternative. 
HNF-SD-TWR-AGA-003

Revision 0

Action: Obtain information from the Private Contractors regarding the range and expected value of the immobilized HLW product loading based on these estimates of the compositions of the enhanced sludge washed feed and the sludge washed feed.

\subsection{SPECIFICATION 8 (WASTE ENVELOPE D)}

The HLW feed specifications are currently undergoing review based on engineering analyses and the results of Private Contractor testing with waste samples and simulants and design analyses. Conclusions in this evaluation regarding the ability for each to satisfy the HLW feed specifications are subject to the final contract specifications, as established for Phase IB.

Action: Incorporate the final HLW feed specifications into this evaluation, when available (May 1998).

\subsection{TANK SPACE IMPLICATIONS}

A conclusion from this analysis is that there will be sufficient DST space available to complete any HLW pretreatment processing alternative with minimal to no impact on cost and schedule. Because the DST space is very tightly constrained during the Phase I HLW pretreatment operations, the HTWOS model results will need to be verified.

Action: Improvements in the accuracy of the HTWOS model are planned for Revision 1 of Kirkbride et al. (1997).

\subsection{HIGH-LEVEL WASTE PROCESSING RATE}

This analysis compares the ability of the each alternative to provide a continuous HLW feed based on the minimum HLW immobilization processing rate (i.e., 60 MT of waste oxides excluding sodium and silicon per year). However, the processing rate is a Private Contractor design uncertainty which has not been established. Therefore, a much higher processing rate above the range analyzed may result in a significant reduction in the "Feed Availability Efficiency" for each alternative, which may influence the preferred pretreatment processing alternative.

Action: Incorporate the final Phase IB contract requirements, when available. 


\subsection{ADDITIONAL HIGH-LEVEL WASTE FEEDS}

Appendix $\mathrm{G}$ of Kirkbride et al. (1997) identifies six additional sources of HLW feed, 241-AY-101, 241-C-101, 241-C-102, 241-C-103, 241-C-104, and 241-C-105, which could potentially be delivered to the Private Contractor during the Phase I optional extension, with minor chemical adjustments or modifications to the HLW feed specifications. These feeds have not been finalized, nor are they included in the current feed delivery plans. If these additional feeds are confirmed to be compliant with the HLW feed specifications, the decision criterion, "Ability to Satisfy the Minimum Order Quantity," is more easily met for all pretreatment processing alternatives.

Action: Confirm that the additional HLW feeds can be delivered to the Private Contractor during Phase I, and will comply with the Phase IB contract requirements and feed specifications. 
HNF-SD-TWR-AGA-003

Revision 0

This page intentionally left blank. 


\section{HNF-SD-TWR-AGA-003 \\ Revision 0}

\subsection{REFERENCES}

Bacon, R. F., 1996, Final Neutralized Current Acid Waste Consolidation Recommendation, letter to J. E. Kinzer, 9651466 (May 10), Westinghouse Hanford Company, Richland, Washington.

Boston, H. L., 1997, Subcontract Number 80232764-9-K001, Interim Issue of Waste Feed Delivery Mid-Level Logics, letter to A. M. Umek, LMHC-9756006 (August 4), Lockheed Martin Hanford Corporation, Richland, Washington.

Calmus, R. B., 1996, Solidified High-Level Waste Interim Storage Alternative Analysis and Path Forward Recommendation, WHC-SD-WM-SP-011, Revision 0, Westinghouse Hanford Company, Richland, Washington.

Claghorn, R. D., J. D. Galbraith, and T. B. Salzano (FDNW), 1997, Alternatives Generation and Analysis for the Phase I Intermediate Waste Feed Staging System Design Requirements, HNF-SD-TWR-AGA-001, Revision 1, Numatec Hanford Corporation, Richland, Washington.

Colton, N. G., 1997, Status Report: Pretreatment Chemistry Evaluation FY 1997--Wash and Leach Factors for the Single-Shell Tank Waste Inventory, Pacific Northwest National Laboratory, Richland, Washington.

DOE, 1996a, TWRS Privatization (BNFL, Inc.) Contract DE-RP06-96RL13308, September 1996, U.S. Department of Energy-Richland Operations Office, Richland, Washington.

DOE, 1996b, TWRS Privatization (LMAES) Contract DE-RP06-96RL13309, September 1996, U.S. Department of Energy-Richland Operations Office, Richland, Washington.

DOE, 1996c, Tank Waste Remediation System Environmental Impact Statement, U.S. Department of Energy-Richland, Operations Office, Richland, Washington.

Grams, W. H., 1995, Double-Shell Tank Retrieval Allowable Heel Trade Analysis, WHC-SD-WM-TA-162, Revision 0, Westinghouse Hanford Company, Richland, Washington.

Hrma, P. R., G. F. Piepel, M. J. Schweiger, D. E. Smith, D. S. Kim, P. E. Redgate, J. D. Vienna, C. A. LoPresti, D. B. Simpson, D. K. Peeler, and M. H. Langowski, 1994, Property/Composition Relationships for Hanford High-Level Waste Glasses Melting at $1150^{\circ} \mathrm{C}$, Volume 1, PNL-10359, Pacific Northwest Laboratory, Richland, Washington. 
Kirkbride, R. A., G. K. Allen, P. J. Certa, A. F. Manuel, R. M. Orme, L. W. Shelton, E. J. Slaathaug, R. S. Wittman, G. T. Maclean (SESC), and D. L. Penwell (SESC), 1997, Tank Waste Remediation System Operation and Utilization Plan, HNF-SD-WM-SP-012, Revision 0, Numatec Hanford Corporation, Richland, Washington.

Kupfer, M. J., A. L. Boldt, B. A. Higley, S. L. Lambert, D. E. Place, R. M. Orme, L. W. Shelton, R. A. Watrous, G. L. Borsheim (Borsheim Associates), N. G. Colton (PNNL), M. D. LeClair (SAIC), W. W. Schulz (W2 Corporation), D. Hedengren and R. T. Winward (Meier Associates), 1997, Interim Report: Best-Basis Inventories of Chemicals and Radionuclides in Hanford Site Tank Waste, WHC-SD-WM-TI-740, Revision 0, Lockheed Martin Hanford Corporation, Richland, Washington.

Lambert, S. L., and G. E. Stegen, 1996, Tank Waste Remediation System Phase I High-Level Waste Feed Processability Assessment Report, WHC-SD-WM-TI-768, Revision 0 , Westinghouse Hanford Company, Richland, Washington.

MacLean, G. T., 1997, Computer Simulation of the Leaching and Washing of Waste in Tanks $C-106, A Y-102, A Z-101$, and $A Z-102$, HNF-SD-TWR-PE-001, Revision 0, SGN Eurisys Services Corporation, Richland, Washington.

Manuel, A. F., S. L. Lambert, and G. E. Stegen, 1996, Phase I High-Level Waste Pretreatment and Feed Staging Plan, WHC-SD-WM-ES-370, Revision 1, Westinghouse Hanford Company, Richland, Washington.

Milner, R. A., 1996, Repository Disposal Fee Estimates for the Hainford TWRS-EIS, letter to J. E. Kinzer, (July 1), Office of Civilian Radioactive Waste Management.

Place, D. E., D. W. Hendrickson, L. A. Fort, G. T. MacLean, and K. M. Eager, 1997, High-Level Waste Feed Capability Assessment, SESC-EN-RPT-008, SGN Eurisys Corporation, Richland, Washington.

Sathyanarayana, K., B. C. Fryer, and M. J. Thurgood, 1994, Summary Report - Thermal Hydraulic Safety Analysis of Aging Waste Tank 101-AZ, WHC-SD-WM-ER-335, Revision 0, Westinghouse Hanford Company, Richland, Washington.

Sterner, S. M., A. R. Felmy, P. K. Melethil, M. J. Mason, and J. R. Rustad, 1996, Extension of the ESP Model to High Base and High Aluminate Concentration, Letter Report (May 31), Pacific Northwest National Laboratory, Richland, Washington. 
HNF-SD-TWR-AGA-003

Revision 0

\section{APPENDIX A}

\section{LIFE-CYCLE COST ESTIMATES FOR THE PHASE I HIGH-LEVEL WASTE PRETREATMENT PROCESSING ALTERNATIVES}


HNF-SD-TWR-AGA-003

Revision 0

This page intentionally left blank. 
HNF-SD-TWR-AGA-003

Revision 0

APPENDIX A

\section{LIFE-CXCLE COST ESTIMATES FOR THE PHASE I HIGH-LEVEX WASTE PRETREATMENT PROCESSING ALTERNATIVES}

This appendix details the calculations of the base case life-cycle costs for each pretreatment processing alternative, based on the flowsheet modeling results. The results of these calculations (i.e., the life-cycle cost comparisons) are plotted in Figures A-2 and A-3. Appendix $B$ discusses the results of sensitivity studies regarding technical uncertainties, such as the waste oxide loading, the high-level waste (HLW) product formulation, and the availability of additional feeds to satisfy the maximum order quantity.

\section{A1.0 LIFE-CYCLE COST EVALUATION}

This section summarizes the flowsheet modeling results and details the calculation of life-cycle costs.

\section{A1.1 LIFE-CYCLE COST EQUATION}

Based on the measurable decision criteria for cost minimization discussed in this report, the following equation is used to calculate the life-cycle costs for each pretreatment processing alternative:

Life-Cycle Cost Equation

$$
\begin{aligned}
& \left(\begin{array}{c}
C_{\text {life }}^{\text {cycle }}
\end{array}\right)_{\text {pretreatment }}=\left(C_{\text {capital }}\right)_{\text {pretreatment }}^{\text {equipment }}+\left(C_{\text {operating }}\right)_{\text {pretreatment }}+
\end{aligned}
$$

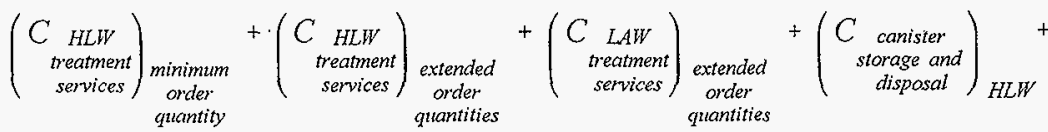

$$
\begin{aligned}
& \left(\begin{array}{c}
C_{\text {onsite }} \\
\text { disposal }
\end{array}\right)_{L A W}+\left(C_{\text {operating }}\right) \begin{array}{c}
\text { decanted } \\
\text { liquids } \\
\text { storage }
\end{array}+\left(C_{\text {capital }}\right)_{\begin{array}{c}
\text { decanted } \\
\text { liquids } \\
\text { storage }
\end{array}}
\end{aligned}
$$


The fourth and fifth terms of the life-cycle cost equation, the costs for processing the extended order quantities of HLW and low-activity waste (LAW) feed, are currently unknown. These costs will be based on the "Fixed-Unit-Prices" for processing extended order quantities yet to be established during Phase IA. Also, if the maximum order quantities of HLW and LAW feed are processed, the cost difference between the alternatives will occur during Phase II, and no information on Phase II Privatization costs is available. Therefore, this evaluation assumes different ratios of the "Fixed-Unit-Prices" for the extended order quantities to the "Target-UnitPrices" for processing the minimum order quantities of HLW and LAW feed (for each Private Contractor).

The last two terms of the life-cycle cost equation, the operating and capital costs for decanted liquids storage, are calculated based on the estimated DST space needs for each pretreatment processing alternative. If no additional DST space is required, the capital cost is zero. The relative differences in operating costs for each alternative are negligible, regardless of whether or not additional DST space is required. These costs are based on the results of the Hanford Tank Waste Operation Simulator (HTWOS) runs, provided in Appendix D.

\section{A1.2 SUMMARY OF FLOWSHEET RESULTS}

The pretreatment process was simulated using two computer programs called the Environmental Simulation Program (ESP ${ }^{1}$ ) and ASPEN PLUS ${ }^{2}$. The ESP model was used to estimate the compositions of the sludges in 241-AZ-101, 241-AZ-102, 241-AY-102, and 241-C-106, and to predict the product compositions after pretreatment. The results of the ESP simulations were used as input to the ASPEN PLUS models, to track the flow of materials and the composition of the sludge for each step of the pretreatment process, as shown in Figure A-1. The detailed material balance tables are provided in Appendix C.

${ }^{1}$ ESP is a registered trademark of OLI Systems, Inc.

${ }^{2}$ ASPEN PLUS is a registered trademark of Aspen Technology, Inc, Cambridge, MA. 


\section{HNF-SD-TWR-AGA-003 \\ Revision 0}

Figure A-1. Waste Movements During Phase I High-Level Waste Pretreatment and Feed Staging.

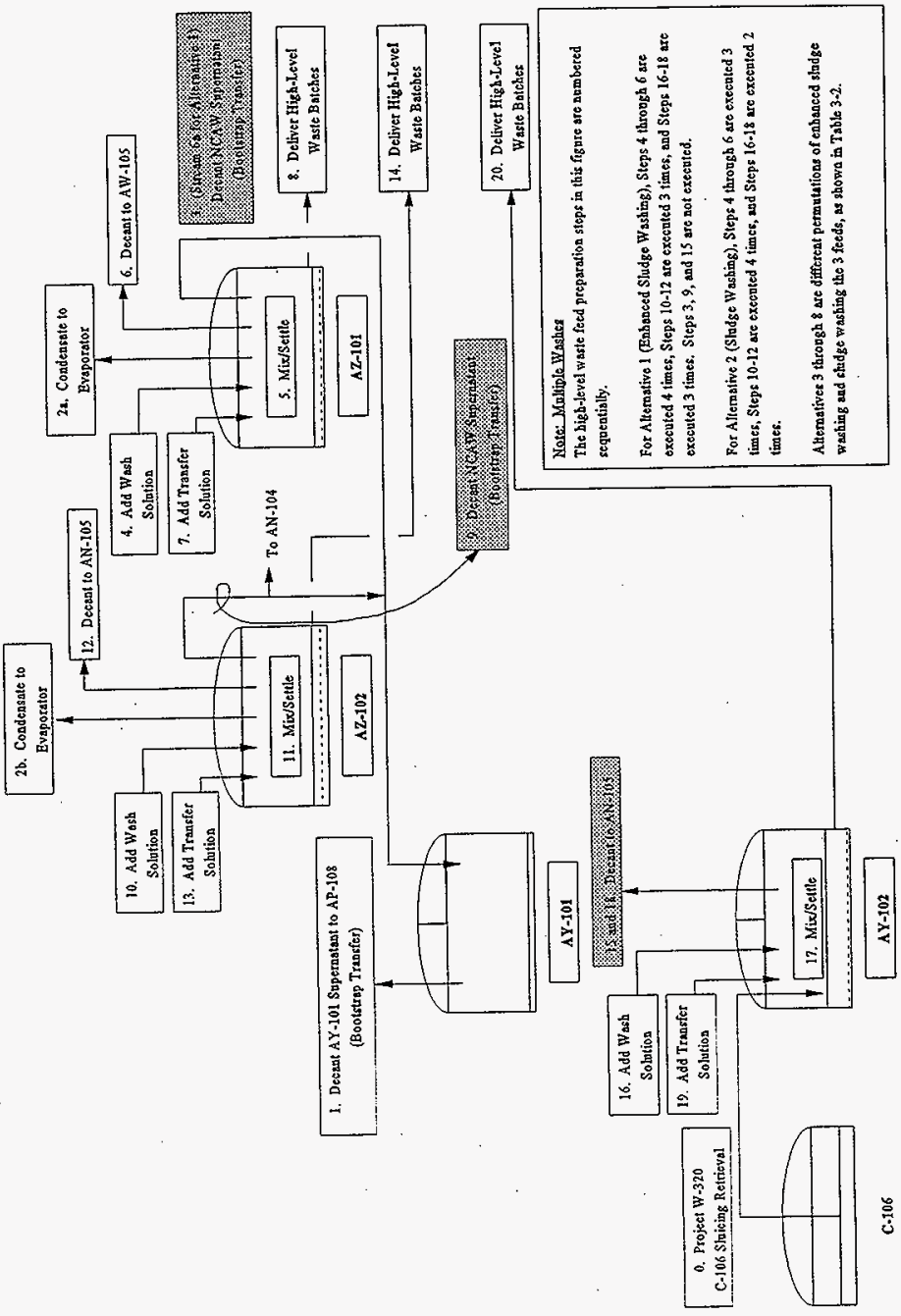




\section{Revision 0}

Tables A-1, A-2, and A-3 summarize the flowsheet results from the material balances in Appendix C.

Table A-1. Summary of Phase I High-Level Waste Pretreatment Flowsheet Results (Alternative 1 - Enhanced Sludge Washing).

\begin{tabular}{|l|c|c|c|c|c|}
\cline { 2 - 6 } \multicolumn{1}{c|}{} & HLW feed & $\begin{array}{c}\text { HLW } \\
\text { canisters }^{\mathrm{b}} \\
\left(1.08-\mathrm{m}^{3}\right.\end{array}$ & $\begin{array}{c}\text { LAW feed } \\
\text { capacity) }^{\mathrm{C}}\end{array}$ & $\begin{array}{c}\text { Volume of } \\
50 \text { wt\% caustic } \\
\text { solution (gal) }\end{array}$ & $\begin{array}{c}\text { Mass of } \\
\text { steam (MT) }\end{array}$ \\
\hline $241-\mathrm{AZ-101}$ & 66.6 & 93 & 396 & 20,200 & TBD \\
\hline $241-\mathrm{AZ-102}$ & 114 & 159 & 258 & 39,300 & TBD \\
\hline $241-\mathrm{AY}-102^{\mathrm{d}}$ & 153 & 213 & 255 & 56,700 & TBD \\
\hline Total & 334 & 464 & 910 & 116,000 & TBD \\
\hline
\end{tabular}

HLW $=$ High-level waste

LAW $=$ Low-activity waste

${ }^{\mathrm{a}} \mathrm{MT}$ of waste oxides excluding sodium and silicon (100 percent retrieval)

${ }^{b}$ Based on $25 \mathrm{w} t \%$ waste oxide loading excluding sodium and silicon

${ }^{\circ}$ Low-activity waste feed resulting from high-level waste pretreatment, including the initial decants from 241-AZ-101 and 241-AZ-102

${ }^{d}$ Includes 100 percent of the sludge from 241 C-106

Table A-2. Summary of Phase I High-Level Waste Pretreatment Flowsheet Results (Alternative 2 - Sludge Washing).

\begin{tabular}{|l|c|c|c|c|c|}
\cline { 2 - 6 } \multicolumn{1}{c|}{} & HLW feed & $\begin{array}{c}\text { HLW } \\
\text { canisters } \\
\left(1.08-\mathrm{m}^{\mathrm{b}}\right.\end{array}$ & $\begin{array}{c}\text { LAW feed } \\
\text { (MT Na) } \\
\text { capacity) }\end{array}$ & $\begin{array}{c}\text { Volume of } \\
50 \text { wt\% caustic } \\
\text { solution (gal) }\end{array}$ & $\begin{array}{c}\text { Mass of } \\
\text { steam (MT) }\end{array}$ \\
\hline $241-\mathrm{AZ}-101$ & 96.4 & 134 & 360 & 2,290 & 0 \\
\hline $241-\mathrm{AZ}-102$ & 162 & 225 & 197 & 4,450 & 0 \\
\hline $241-\mathrm{AY}-102^{d}$ & 194 & 270 & 161 & 3,390 & 0 \\
\hline Total & 452 & 629 & 717 & 10,100 & 0 \\
\hline
\end{tabular}

HLW $=$ High-level waste

LAW $=$ Low-activity waste

${ }^{2} \mathrm{MT}$ of waste oxides excluding sodium and silicon (100 percent retrieval)

based on $25 \mathrm{wt} \%$ waste oxide loading excluding sodium and silicon

'Low-activity waste feed resulting from high-level waste pretreatment, including the initial decants from 241-AZ-101 and 241-AZ-102

${ }^{d}$ Includes 100 percent of the sludge from $241-\mathrm{C}-106$. 
Revision 0

Table A-3. Volumes of Decanted Liquids Resulting from Pretreatment Processing.

\begin{tabular}{|l|c|c|c|c|c|c|c|c|}
\cline { 2 - 8 } \multicolumn{1}{c|}{} & \multicolumn{7}{c|}{ Alternative } \\
\cline { 2 - 8 } \multicolumn{1}{c|}{} & 1 & 2 & 3 & 4 & 5 & 6 & 7 & 8 \\
\hline $\begin{array}{l}\text { Before evaporation } \\
\text { (kgal) }\end{array}$ & 4,010 & 1,810 & 3,370 & 3,320 & 3,150 & 2,500 & 2,670 & 2,450 \\
\hline $\begin{array}{l}\text { After evaporation } \\
\text { (kgal), }\end{array}$ & 1,490 & 304 & 1,180 & 957 & 1,140 & 833 & 648 & 613 \\
\hline
\end{tabular}

"The decanted liquid volumes exclude the "initial decants" from 241-AZ-101 and 241-AZ-102, which are the neutralized current acid waste supernatants designated for Waste Envelope B feed to low-activity waste immobilization.

${ }^{b}$ Evaporation to a $7 M$ Na concentration.

The flowsheet data for Alternatives 3 through 8 are different combinations of the values in Tables A-1 through A-2, assuming enhanced sludge washing or sludge washing for each tank.

\section{A1.3 COST ESTIMATES}

Table A-4 lists the cost estimate tables which follow. 


\section{Revision 0}

Table A-4. List of Cost Estimate Tables.

\begin{tabular}{|c|l|}
\hline Table & \multicolumn{1}{|c|}{ Title } \\
\hline A-5 & Estimated Storage and Disposal Costs. \\
\hline A-6 & $\begin{array}{l}\text { Summary of Phase I Pretreatment and Disposal Costs by Tank (Based on } \\
\text { Enhanced Sludge Washing). }\end{array}$ \\
\hline A-7 & $\begin{array}{l}\text { Summary of Phase I Pretreatment and Disposal Costs by Tank (Based on Sludge } \\
\text { Washing). }\end{array}$ \\
\hline A-8 & $\begin{array}{l}\text { Summary of Phase I Pretreatment and Diposal Costs by Pretreatment Processing } \\
\text { Alternative. }\end{array}$ \\
\hline A-9 & \begin{tabular}{l} 
Phase IA Target-Unit-Prices. \\
\hline A-10
\end{tabular} $\begin{array}{l}\text { High-Level Waste Immobilization Costs (Based on BNFL, Inc. "Target-Unit- } \\
\text { Prices"). (6 Sheets) }\end{array}$ \\
\hline A-11 & $\begin{array}{l}\text { High-Level Waste Immobilization Costs (Based on LMAES "Target-Unit- } \\
\text { Prices"). (6 Sheets) }\end{array}$ \\
\hline A-12 & $\begin{array}{l}\text { Low-Activity Waste Immobilization Costs (Based on BNFL, Inc. "Target-Unit- } \\
\text { Prices"). }\end{array}$ \\
\hline A-13 & $\begin{array}{l}\text { Low-Activity Waste Immobilization Costs (Based on LMAES "Target-Unit- } \\
\text { Prices"). }\end{array}$ \\
\hline A-14 & $\begin{array}{l}\text { Life-cycle Costs for Phase I Pretreatment Processing Alternatives (Based on } \\
\text { BNFL, Inc. "Target-Unit-Prices" for HLW and LAW Services). }\end{array}$ \\
\hline A-15 & $\begin{array}{l}\text { Life-cycle Costs for Phase I Pretreatment Processing Alternatives (Based on } \\
\text { LMAES "Target-Unit-Prices" for HLW and LAW Services). }\end{array}$ \\
\hline
\end{tabular}

BNFL $=$ British Nuclear Fuels Limited

HLW $=$ High-level waste

LAW $=$ Low-activity waste

LMAES $=$ Lockheed Martin Advanced Environmental Services. 
Table A-5. Estimated Storage and Disposal Costs.

\begin{tabular}{|c|c|}
\hline High-Level Waste Canister Incremental Interim Storage Cost & $\$ 65,000$ \\
\hline $\begin{array}{c}\text { High-Level Waste Canister Incremental Disposal Cost } \\
\text { (per 4.5-m [15-ft] canister) }\end{array}$ & $\$ 422,000$ \\
\hline Low-Activity Waste Incremental Disposal Cost (per MT Na) & $\$ 12,077$ \\
\hline
\end{tabular}

${ }^{a}$ Based on Calmus (1996)

'Based on DOE (1996c)

'Based on FM-20 cost estimate.

Table A-6. Summary of Phase I Pretreatment and Disposal Costs by Tank (Based on Enhanced Sludge Washing).

\begin{tabular}{|c|c|c|c|c|c|}
\hline & \multicolumn{2}{|c|}{ Pretreatment } & \multicolumn{2}{c|}{ HLW canisters } & \multirow{2}{*}{ LAW disposal } \\
\cline { 2 - 6 } & Capital cost & $\begin{array}{c}\text { Operating } \\
\text { cost }^{\mathbf{a}}\end{array}$ & $\begin{array}{c}\text { Interim } \\
\text { storage cost }\end{array}$ & Disposal cost & \\
\hline $241-\mathrm{AZ}-101$ & $\$ 25,000,000$ & $\$ 3,890,230$ & $\$ 6,027,569$ & $\$ 39,132,832$ & $\$ 4,784,907$ \\
\hline $241-\mathrm{AZ}-102$ & $\$ 25,000,000$ & $\$ 3,918,994$ & $\$ 10,317,460$ & $\$ 66,984,127$ & $\$ 3,119,489$ \\
\hline $241-\mathrm{AY}-102^{\mathbf{b}}$ & $\$ 25,000,000$ & $\$ 3,945,017$ & $\$ 13,838,067$ & $\$ 89,840,991$ & $\$ 3,083,258$ \\
\hline Total & $\$ 75,000,000$ & $\$ 11,754,240$ & $\$ 30,183,097$ & $\$ 195,957,950$ & $\$ 10,987,655$ \\
\hline
\end{tabular}

HLW $=$ High-level waste

LAW $=$ Low-activity waste

${ }^{a}$ Based on estimated operating costs from Claghorn et al. (1997) plus consumables: 50 wt\% caustic ( $\$ 1.50 /$ gallon), steam at $15 \mathrm{MPa}(\$ 5.00 / \mathrm{MT})$.

${ }^{b}$ Includes 100 percent of the sludge from 241-C-106:

Table A-7: Summary of Phase I Pretreatment and Disposal Costs by Tank (Based on Sludge Washing).

\begin{tabular}{|c|c|c|c|c|c|}
\hline & \multicolumn{2}{|c|}{ Pretreatment } & \multicolumn{2}{c|}{ HLW Canisters } & \multirow{2}{*}{$\begin{array}{c}\text { LAW disposal } \\
\text { cost }\end{array}$} \\
\cline { 2 - 5 } & Capital cost & $\begin{array}{c}\text { Operating } \\
\text { cost }^{\mathrm{a}}\end{array}$ & $\begin{array}{c}\text { Interim } \\
\text { storage cost }\end{array}$ & Disposal cost & \\
\hline $241-\mathrm{AZ}-101$ & $\$ 23,000,000$ & $\$ 3,860,000$ & $\$ 8,724,589$ & $\$ 56,642,718$ & $\$ 4,341,682$ \\
\hline $241-\mathrm{AZ}-102$ & $\$ 23,000,000$ & $\$ 3,860,000$ & $\$ 14,616,402$ & $\$ 94,894,180$ & $\$ 2,377,961$ \\
\hline $241-\mathrm{AY}-102^{\mathrm{b}}$ & $\$ 23,000,000$ & $\$ 3,860,000$ & $\$ 17,539,683$ & $\$ 113,873,016$ & $\$ 1,943,189$ \\
\hline Total & $\$ 69,000,000$ & $\$ 11,580,000$ & $\$ 40,880,674$ & $\$ 265,409,914$ & $\$ 8,662,832$ \\
\hline
\end{tabular}

$\mathrm{HLW}=$ High-level waste

LAW $=$ Low-activity waste

${ }^{2}$ Based on estimated operating costs from Claghorn et al. (1997) plus consumables: $50 \mathrm{wt} \%$ caustic $(\$ 1.50 /$ gallon), steam at $15 \mathrm{MPa}(\$ 5.00 / \mathrm{MT})$.

bincludes 100 percent of the sludge from 241-C-106. 
Table A-8. Summary of Phase I Pretreatment and Disposal Costs by Pretreatment Processing Alternative.

\begin{tabular}{|c|c|c|c|c|c|}
\hline & \multicolumn{2}{|c|}{ Pretreatment } & \multicolumn{2}{c|}{ HLW canisters } & \multirow{2}{*}{$\begin{array}{c}\text { LAW disposal } \\
\text { cost }\end{array}$} \\
\cline { 2 - 6 } & Capital cost & $\begin{array}{c}\text { Operating } \\
\text { cost }^{\mathrm{a}}\end{array}$ & $\begin{array}{c}\text { Interim } \\
\text { storage cost }\end{array}$ & Disposal dost & \\
\hline Alternative 1 & $\$ 75,000,000$ & $\$ 11,754,240$ & $\$ 30,183,097$ & $\$ 195,957,950$ & $\$ 10,987,655$ \\
\hline Alternative 2 & $\$ 69,000,000$ & $\$ 11,580,000$ & $\$ 40,880,674$ & $\$ 265,409,914$ & $\$ 8,662,832$ \\
\hline Alternative 3 & $\$ 73,000,000$ & $\$ 11,669,223$ & $\$ 33,884,712$ & $\$ 219,989,975$ & $\$ 9,847,586$ \\
\hline Alternative 4 & $\$ 73,000,000$ & $\$ 11,724,011$ & $\$ 32,880,117$ & $\$ 213,467,836$ & $\$ 10,544,429$ \\
\hline Alternative 5 & $\$ 73,000,000$ & $\$ 11,695,247$ & $\$ 34,482,038$ & $\$ 223,868,003$ & $\$ 10,246,127$ \\
\hline Alternative 6 & $\$ 71,000,000$ & $\$ 11,610,230$ & $\$ 38,183,654$ & $\$ 247,900,028$ & $\$ 9,106,058$ \\
\hline Alternative 7 & $\$ 71,000,000$ & $\$ 11,638,994$ & $\$ 36,581,732$ & $\$ 237,499,861$ & $\$ 9,404,360$ \\
\hline Alternative 8 & $\$ 71,000,000$ & $\$ 11,665,017$ & $\$ 37,179,059$ & $\$ 241,377,889$ & $\$ 9,802,901$ \\
\hline
\end{tabular}

HLW $=$ High-level waste

LAW $=$ Low-activity waste

aBased on estimated operating costs from Claghorn et al. (1997) plus consumables: $50 \mathrm{wt} \%$ caustic ( $\$ 1.50 /$ gallon), steam at $15 \mathrm{MPa}(\$ 5.00 / \mathrm{MT})$.

${ }^{b}$ Includes 100 percent of the sludge from 241-C-106.

Table A-9. Phase IA Target-Unit-Prices.

\begin{tabular}{|l|c|c|}
\hline Phase IA contract & Cost per MT of HLW feed & Cost per MT of LAW feed \\
\hline BNFL, Inc. & $\$ 1,045,000$ & $\$ 1,040,000$ \\
\hline LMAES & $\$ 7,259,300$ & $\$ 767,200$ \\
\hline
\end{tabular}

$\mathrm{BNFL}=$ British Nuclear Fuels Limited

HLW $=$ High-level waste

LAW $=$ Low-activity waste

LMAES $=$ Lockheed Martin Advanced Environmental Services

${ }^{2} \mathrm{MT}$ of non-volatile oxides excluding sodium and silicon

${ }^{b} \mathrm{MT}$ of sodium

"From DOE (1996a)

${ }^{d}$ From DOE (1996b). 
HNF-SD-TWR-AGA-003

Revision 0

Table A-10. High-Level Waste Immobilization Costs

(Based on BNFL, Inc. "Target-Unit-Prices").

(Sheet 1 of 6 )

\begin{tabular}{|c|c|c|c|c|c|c|}
\hline & \multicolumn{4}{|c|}{ Immobilization Cost Ratio (for HLW and LAW Services) = } & \multicolumn{2}{|l|}{0.0} \\
\hline & 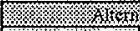 & 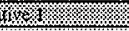 & & & 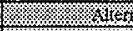 & 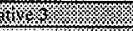 \\
\hline & 6\% & dyodo & Whinow & 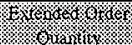 & 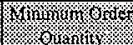 & Wrow \\
\hline AZ-101 & $\$ 69,597,000$ & \$o & $\$ 100,738,000$ & \$o & $\$ 69, \$ 97,000$ & $\$ 0$ \\
\hline $\mathrm{AZ}-102$ & $\$ 119,130,000$ & $\$ 0$ & $\$ 155,287,000$ & so & $\$ 119,130,000$ & $\$ 0$ \\
\hline$A Y-102$ & $\$ 67,298,000$ & $\$ 0$ & $\$ 0$ & so & $\$ 67,298,000$ & $\$ 0$ \\
\hline Total & $\$ 256,025,000$ & $\$ 0$ & $\$ 256,025,000$ & so & $\$ 256,025,000$ & so \\
\hline
\end{tabular}

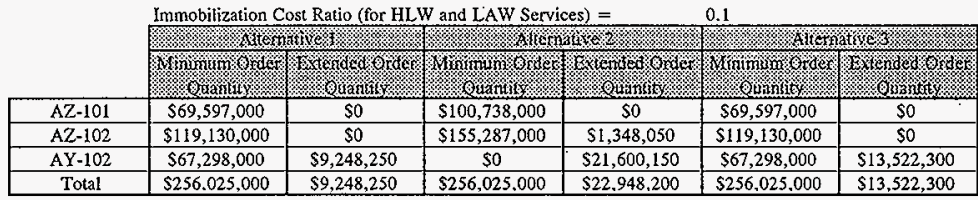

Inmobilization Cost Ratio (for HLW and LAW Services) =

0.2

\begin{tabular}{|c|c|c|c|c|c|c|}
\hline & \multicolumn{4}{|c|}{ Inmobilization Cost Ratio (for HLW and LAW Services) $=$} & \multicolumn{2}{|l|}{0.2} \\
\hline & \multicolumn{2}{|c|}{ V } & \multicolumn{2}{|c|}{ 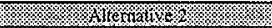 } & \multicolumn{2}{|c|}{ N } \\
\hline & Whinning & 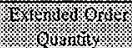 & Whin & How & Gringinoring & 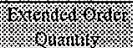 \\
\hline AZ-101 & $\$ 69,597,000$ & so & $\$ 100,738,000$ & so & $\$ 69,597,000$ & $\$ 0$ \\
\hline $\mathrm{AZ}-102$ & $\$ 119,130,000$ & \$0 & $\$ 155,287,000$ & $\$ 2,696,100$. & $\$ 119,130,000$ & $\$ 0$ \\
\hline $\mathrm{AY}-102$ & $\$ 67,298,000$ & $\$ 18,496,500$ & \$50 & $\$ 43,200,300$ & $\$ 67,298,000$ & $\$ 27,044,600$ \\
\hline Total & $\$ 256,025,000$ & $\$ 18,496,500$ & $\$ 256,025,000$ & $\$ 45,896,400$ & $\$ 256,025,000$ & $\$ 27,044,600$ \\
\hline
\end{tabular}

\begin{tabular}{|c|c|c|c|c|c|c|}
\hline & \multicolumn{4}{|c|}{ Immobilization Cost Ratio (for HLW and LAW Services) = } & \multicolumn{2}{|l|}{0.3} \\
\hline & V) & Who 6 \% & 楼 & (3) & 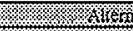 & $x_{2}$ \\
\hline & 繁 & 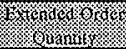 & H) & 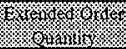 & Y) & Wrong \\
\hline$A Z-101$ & $\$ 69,597,000$ & so & $\$ 100,738,000$ & so & $\$ 69,597,000$ & so \\
\hline$A Z-102$ & $\$ 119,130,000$ & $\$ 0$ & $\$ 155,287,000$ & $\$ 4,044,150$ & $\$ 119,130,000$ & $\$ 0$ \\
\hline$A Y-102$ & $\$ 67,298,000$ & $\$ 27,744,750$ & $\$ 0$ & $\$ 64,800,450$ & $\$ 67,298,000$ & $\$ 40,566,900$ \\
\hline Total & $\$ 256.025,000$ & $\$ 27,744.750$ & $\$ 256.025,000$ & $568.844,600$ & $\$ 256.025 .000$ & $\$ 40,566.900$ \\
\hline
\end{tabular}

\begin{tabular}{|c|c|c|c|c|c|c|}
\hline & \multicolumn{4}{|c|}{ Immobilization Cost Ratio (for HLW and LAW Services) = } & \multicolumn{2}{|l|}{0.4} \\
\hline & \multicolumn{2}{|c|}{ W r } & \multicolumn{2}{|c|}{ Ky. } & \multicolumn{2}{|c|}{ 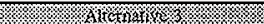 } \\
\hline & WGininingrow & 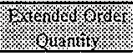 & Winh Monding & 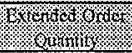 & 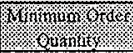 & 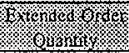 \\
\hline $\mathrm{AZ}-101$ & $\$ 69,597,000$ & $\$ 0$ & $\$ 100,738,000$ & so & $\$ 69,597,000$ & So \\
\hline $\mathrm{AZ}-102$ & $\$ 119,130,000$ & So & $\$ 155,287,000$ & $\$ 5,392,200$ & $\$ 119,130,000$ & \$o \\
\hline $\mathrm{AY}-102$ & $\$ 67,298,000$ & $\$ 36,993,000$ & $\$ 0$ & $\$ 86,400,600$ & $\$ 67,298,000$ & $\$ 54,089,200$ \\
\hline Total & $\$ 256,025,000$ & $\$ 36,993,000$ & $\$ 256,025,000$ & $\$ 91,792.800$ & $\$ 256.025 .000$ & $\$ 54,089,200$ \\
\hline
\end{tabular}

\begin{tabular}{|c|c|c|c|c|c|c|}
\hline & \multicolumn{4}{|c|}{ Inmobilization Cost Ratio (for HLW and LAW Services) $=$} & \multicolumn{2}{|l|}{0.5} \\
\hline & \multicolumn{2}{|c|}{ 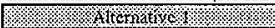 } & \multicolumn{2}{|c|}{ 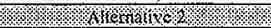 } & \multicolumn{2}{|c|}{ 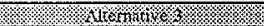 } \\
\hline & Wininning & Whordorid & Whonown & Exrendedo & Minmunowor & Whow \\
\hline$A Z-101$ & $\$ 69,597,000$ & SO & $\$ 100,738,000$ & so & $\$ 69,597,000$ & $\$ 0$ \\
\hline $\mathrm{AZ}-102$ & $\$ 119,130,000$ & \$0 & $\$ 155,287,000$ & $\$ 6,740,2$ & $\$ 119,130,000$ & $\$ 0$ \\
\hline $\mathrm{AY}-102$ & $\$ 67,298,000$ & $\$ 46,241,250$ & $\$ 0$ & $\$ 108,000,750$ & $567,298,000$ & $\$ 67,611,500$ \\
\hline Total & $\$ 256,025,000$ & $\$ 46.241 .250$ & $\$ 256.025 .000$ & $5114.741,0.00$ & $\$ 256.025 .000$ & $\$ 67,611,500$ \\
\hline
\end{tabular}




\begin{tabular}{|c|c|c|c|c|c|c|}
\hline $0 \varsigma Z^{2} 0 \varepsilon t^{\prime} Z 6 S$ & $000^{\circ} \subseteq 20^{\circ} 9525$ & $000^{\circ} 090^{\circ} I L S$ & $000^{\circ} 520^{\circ} 952 S$ & OSL'II8.19S & $000^{\prime} S 20^{\circ} 9 S 2 S$ & [010 I \\
\hline $0 \varsigma z^{\circ} 0 \varepsilon t^{\prime} 26 \varsigma$ & $005^{\circ} 099^{\circ} \angle T S$ & $000^{\circ} 090^{\circ} \backslash \mathrm{LS}$ & $005^{\circ} 09^{\circ} \mathrm{LIS}$ & OSL'II8'I9S & $000^{\circ}$ LSIO 9 ES & $\mathrm{ZOI}-\mathrm{XV}$ \\
\hline $0 s$ & $00 S^{\circ} \angle 9 L 891 S$ & OS & $00 S^{\prime} \angle 9 L^{\prime} 891 \mathrm{~S}$ & OS & $000^{\circ} 08$ I $^{\circ} 6$ IIS & COI-ZV \\
\hline OS & $000^{2} \angle 65695$ & OS & $000^{\circ} \angle 6 S^{\prime} 69 S$ & OS & 00082 E.00IS & $10 r-7 v$ \\
\hline 4kinup & W31361 & 110 & 两1 & SMMg & 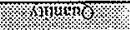 & \\
\hline 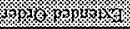 & gNomonum & (2010 & 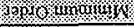 & $103<0,11519$ & $6 \times 1,40140$ & \\
\hline \% & Horn & Sh & 4231 & 13318 & 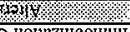 & \\
\hline
\end{tabular}

\begin{tabular}{|c|c|c|c|c|c|c|}
\hline $00 z^{\prime}+666^{\prime} \mathcal{L} L S$ & $000^{\circ} \$ 20^{\circ} 9 \leq 2 S$ & $000878^{\circ} 9 \$ \$$ & $000^{\circ} \$ 20^{\circ} 9 \varsigma 2 \$$ & $000^{\circ} 606^{\circ} 605$ & $000^{\circ} s 20^{\circ} 9 s 25$ & 1010J \\
\hline $00 \tau^{\prime}+66^{\prime} \varepsilon L S$ & $005^{\circ} 099^{\circ}$ is & $000^{\circ} 808^{\circ} 955$ & $005^{\circ} 099^{\circ} \mathrm{LIS}$ & $00 b^{\prime} 6 b^{\prime} 605$ & $000^{\circ} L 5 I^{\circ} 9 E S$ & $201-X V$ \\
\hline $0 \$$ & $005^{\circ} \angle 9 L^{\prime} 891 \mathrm{~S}$ & $0 \hat{S}$ & $0^{\circ} \angle 9 L^{\circ} 891 \mathrm{~S}$ & $0 S$ & $000^{\circ} 0 \varepsilon I^{\circ} 6115$ & $20 T-Z V$ \\
\hline $0 S$ & $000 \angle 65695$ & os & $000^{\circ} \angle 65^{6} 69 \mathrm{~S}$ & $0 \mathrm{~s}$ & $000^{\circ} 8 E L^{\circ} 001 S$ & $10 \mathrm{~T}-\mathrm{ZV}$ \\
\hline \% & 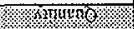 & 410 & 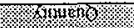 & 14128 & 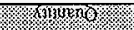 & \\
\hline 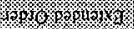 & 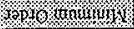 & 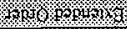 & 201\% & 100102010121 & $40 \%$ \%ư 1 & \\
\hline Kk & 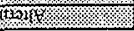 & V(1. & $1 \%$ & 180 & mish & \\
\hline
\end{tabular}

\begin{tabular}{|c|c|c|c|c|c|c|}
\hline OSI'8St'SSS & $000^{\circ} \subseteq 20^{\circ} 9525$ & $000^{\circ} 989^{\circ} \mathrm{zts}$ & $000 \$ 209525$ & OSO $\angle 80^{\circ} \angle E S$ & $000^{\circ} 520^{\circ} 9525$ & TPIOL \\
\hline OSI'8St'SSS & $00 S^{\circ} 099^{\circ} \mathrm{LIS}$ & $000^{\circ} 9 \varepsilon 9^{\circ} \mathrm{ZDS}$ & $005^{\prime} 099^{\circ} \mathrm{LIS}$ & OSO $\angle 80^{\circ} L E S$ & $000^{\circ} \angle S I^{\circ} 9 E S$ & $20 I-\lambda V$ \\
\hline OS & $00 S^{2} \angle 9 L^{\circ} 891 S$ & OS & $00 S^{\prime} \angle 9 L^{\prime} 8915$ & OS & $000^{\circ} 0$ EI 6 IIS & $20 \mathrm{I}-\mathrm{ZY}$ \\
\hline $0 \$$ & $000^{6} \angle 65^{6} 695$ & 05 & $000^{\circ} \angle 6 S^{\circ} 69 S$ & $0 \mathrm{~S}$ & $000^{\circ} 8 \varepsilon L^{\prime} 00 I \$$ & I0 $\mathrm{I}^{\mathrm{Z}} \mathrm{Z}$ \\
\hline \% & 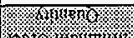 & \% & F & 8 \% & 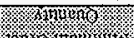 & \\
\hline \%or s & 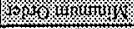 & $90 \% 1 \%$ & H & $8091 \%$ & 3rin & \\
\hline $3 x$ & ystu & 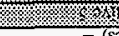 & Wusy & 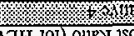 & Wort & \\
\hline
\end{tabular}

\begin{tabular}{|c|c|c|c|c|c|c|}
\hline $001^{\prime} Z \angle \sigma^{\circ} 98 S$ & $000^{\circ} \varsigma 20^{\circ} 9 \varsigma Z S$ & $000^{\circ} \triangleright z t^{\prime} 8 z s$ & $000^{\circ} 520^{\circ} 952 \$$ & $00 L \square Z L \perp Z S$ & $000^{\prime} 520.9525$ & [E]OL \\
\hline $00 I^{\prime} Z L 6^{\prime} 9 E S$ & $005^{\circ} 099^{\circ} \mathrm{LIS}$ & $000^{c} t z t^{c} 8 z S$ & $005^{\prime} 099^{\prime} \mathrm{LIS}$ & $00 L^{\prime} \triangleright Z L^{\prime} \triangleright Z S$ & $000^{\prime}$ LSI'9ES & $20 I-X V$ \\
\hline $0 \$$ & $00 S^{c} \angle 9 L^{\prime} 891 S$ & $0 \mathrm{~s}$ & $00 S^{\prime} \angle 9 L^{\prime} 89 \mathrm{IS}$ & OS & $000^{\circ} 08 I^{\circ} 6$ IS & $20 I-Z V$ \\
\hline $0 S$ & $000^{2} \angle 6 S^{2} 69 S^{\circ}$ & $0 \mathrm{~s}$ & $000^{\circ} \angle 6 S^{\circ} 695$ & $0 S$ & $000^{\circ} 8 \varepsilon L^{\prime} 001 S$ & $10 \mathrm{I}-\mathrm{ZV}$ \\
\hline 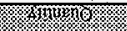 & 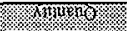 & 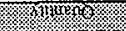 & (6) & 6) & sesurto & \\
\hline $80038 \%$ & 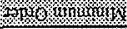 & (1) & Why & $130,010 \%$ & Sop 10 w & \\
\hline \% & Molgrg & 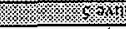 & 115118 & 3483 & $3018 \%$ & \\
\hline
\end{tabular}

\begin{tabular}{|c|c|c|c|c|c|c|}
\hline $050^{\circ} 986^{\circ} 81 \mathrm{~s}$ & $000^{\circ} s 20^{\circ} 9 s 2 S$ & $000212^{2}+15$ & $000^{\circ} \$ 20^{\circ} 952 s$ & 0SE' $29 \varepsilon^{\prime} Z I \$$ & $000^{\circ} \varsigma 20^{\circ} 9 \varsigma 25$ & IE]OL \\
\hline $050^{\circ} 988^{\circ} 815$ & $005^{\circ} 099^{\circ} \mathrm{LIS}$ & $000^{2} z\left[z^{2}+1 S\right.$ & $005^{\circ} 099^{\circ}$ LIS & osร' $29 \varepsilon^{\prime} Z I S$ & $000^{6} \angle S Y^{\circ} 9 E S$ & $20 I-X Y$ \\
\hline $0 S$ & $00 S^{\circ} \angle 9 L^{\circ} 89$ IS & $0 \mathrm{~s}$ & $00 S^{\circ} \angle 9 L^{\circ} 891 S$ & OS & $000^{\circ} 0 \mathrm{SI}^{\circ} 61 \mathrm{IS}$ & $20 \mathrm{t}-\mathrm{ZV}$ \\
\hline $0 \$$ & $000^{\circ} \angle 6 S^{\circ} 69 S$ & $0 S$ & $000^{\circ} \mathrm{L65} 69 \mathrm{~S}$ & OS & $000^{\circ} 8 \varepsilon L^{\circ} 001 S$ & $\mathrm{IOI}-\mathrm{ZV}$ \\
\hline 9. & W' & 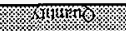 & 6ripno & 65imens & 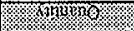 & \\
\hline 6.3. & 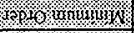 & 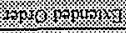 & 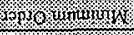 & 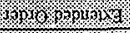 & 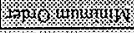 & \\
\hline kx & $11) \times 1 \%$ \% & S\% & Whin & W. & His 18 & \\
\hline
\end{tabular}

\begin{tabular}{|c|c|c|c|c|c|c|}
\hline $0 S$ & $000^{\circ} \subseteq 20^{\circ} 9 \varsigma 2 S$ & $0 S$ & $000^{\circ} 520^{\circ} 9 s 25$ & os & $000^{\circ}$ s20 $^{\circ} 952 \$$ & एकिI \\
\hline OS & $00 S^{\circ} 099^{\circ} \mathrm{LIS}$ & 0 S & $00 S^{\prime} 099^{\prime} \mathrm{LIS}$ & os & $000^{\circ} \angle S I^{\prime} 9 E S$ & $20 T-X V$ \\
\hline os & $00 S^{6} \angle 9 L^{6} 89 \mathrm{IS}$ & $0 \$$ & $0 \mathrm{~S}^{\mathrm{C}} \angle 9 L^{\circ} 89 \mathrm{IS}$ & OS & $000^{\circ} 0$ LI'6IIS & $20 I-Z V$ \\
\hline $0 \$$ & $000^{\circ} \angle 6 S^{\circ} 69 \$$ & $0 S$ & $000^{\circ} \angle 6 S^{\circ} 69 S$ & os & $000^{\prime} 8 \subseteq L^{\prime} 00$ IS & $101-Z \mathrm{~V}$ \\
\hline 4. & Whymo & (W) & 6 6. & 4 & 6yustse & \\
\hline $401 \%$ oroup 4 & 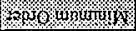 & 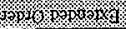 & 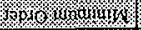 & 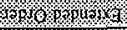 & 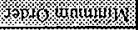 & \\
\hline \% & $\mathrm{Hrrin}_{2}$ & K & $182314 \%$ & \%र\% & 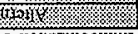 & \\
\hline
\end{tabular}

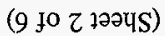

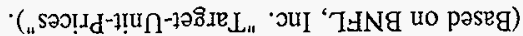

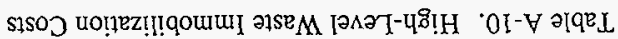 \\ 0 Uоก!ฺ! \\ $\varepsilon 00-\forall D V^{-}-\mathrm{M} L-G S^{-} \mathrm{JNH}$
}


HNF-SD-TWR-AGA-003

Revision 0

Table A-10. High-Level Waste Immobilization Costs

(Based on BNFL, Inc. "Target-Unit-Prices").

(Sheet 3 of 6)

\begin{tabular}{|c|c|c|c|c|}
\hline & \multicolumn{4}{|c|}{ Immobilization Cost Ratio (for HLW and LAW Services) $=$} \\
\hline & \multicolumn{2}{|c|}{ 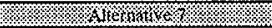 } & \multicolumn{2}{|c|}{ F. } \\
\hline & 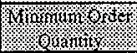 & 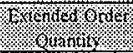 & Whonn & oroworom \\
\hline $\mathrm{AZ}-101$ & $\$ 100,738,000$ & \$0 & $\$ 100,738,000$ & $\$ 0$ \\
\hline $\mathrm{AZ}-102$ & $\$ 119,130,000$ & so & $\$ 155,287,000$ & $\$ 0$ \\
\hline $\mathrm{AY}-102$ & $\$ 36,157,000$ & so & \$0 & So \\
\hline Total & $\$ 256.025 .000$ & so & $\$ 256.025 .000$ & So \\
\hline
\end{tabular}

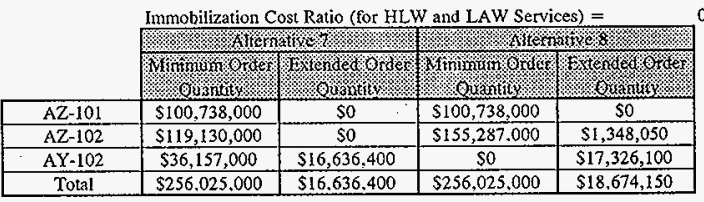

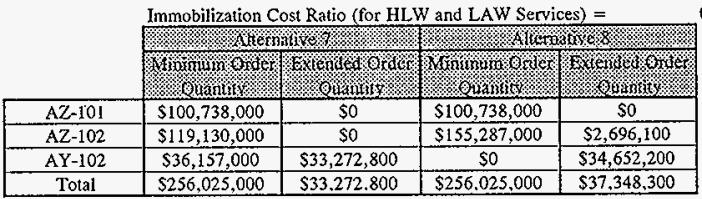

\begin{tabular}{|c|c|c|c|c|}
\hline & \multicolumn{4}{|c|}{ Immobilization Cost Ratio (for HLW and LAW Services) = } \\
\hline & \multicolumn{2}{|c|}{ 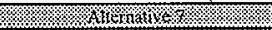 } & \multicolumn{2}{|c|}{ (7) } \\
\hline & Wrin & Whor & Winow & Wrid \\
\hline$A Z-101$ & $\$ 100,738,000$ & $\$ 0$ & $\$ 100,738,000$ & $\$ 0$ \\
\hline$A Z-102$ & $\$ 119,130,000$ & $\$ 0$ & $\$ 155,287,000$ & $\$ 4,044,150$ \\
\hline $\mathrm{AX}-102$ & $\$ 36,157,000$ & $\$ 49,909,200$ & $\$ 0$ & $\$ 51,978,300$ \\
\hline Total & $\$ 256.025 .000$ & $\$ 49.909 .200$ & $\$ 256.025 .000$ & $\$ 56,022,450$ \\
\hline
\end{tabular}

\begin{tabular}{|c|c|c|c|c|}
\hline & \multicolumn{4}{|c|}{ Immobilization Cost Ratio (for HLW and LAW Services) $=$} \\
\hline & \multicolumn{2}{|c|}{ 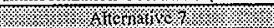 } & \multicolumn{2}{|c|}{ 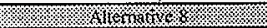 } \\
\hline & 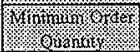 & Honderorom & W10 & 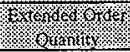 \\
\hline 1 & $\$ 100,738,000$ & $\$ 0$ & $\$ 100,738,000$ & $\$ 0$ \\
\hline $\mathrm{AZ}-102$ & 1,000 & \$0 & $\$ 155,287,000$ & $\$ 5,392,200$ \\
\hline$A Y-102$ & $\$ 36,157,000$ & $\$ 66,545,600$ & so & $\$ 69,304,400$ \\
\hline Total & $\$ 256.025 .000$ & $\$ 66.545 .600$ & $\$ 256.025 .000$ & $\$ 74,696,600$ \\
\hline
\end{tabular}

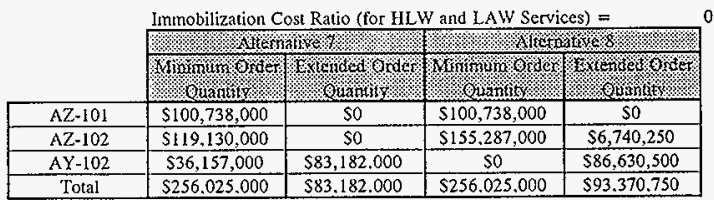




\begin{tabular}{|c|c|c|c|c|c|c|}
\hline $000^{\circ}$ EZZ' ŞEIS & $000^{\circ} \varsigma 20^{\circ} 9 s 2 S$ & $000^{\circ} 28 t^{\circ} 6225$ & $000^{\circ} 520^{\circ} 9525$ & $005^{\circ} 285^{\circ} 265$ & $000^{\circ} 520^{\circ} 952 S$ & [E]OX \\
\hline $000^{\prime} \varepsilon Z Z^{\prime} S E I S$ & $000^{\circ} 86 Z^{\circ} \angle 9 S$ & $005^{\prime} 100^{\circ} 912 S$ & $0 \mathrm{~s}$ & $005^{2} 285^{\circ} 26 s^{\circ}$ & $000^{\circ} 862^{i} \angle 95$ & $201-X V$ \\
\hline $0 \$$ & $000^{\circ} 0$ OEI 6 IIS & $00 S^{\prime} 08 t^{\prime} \varepsilon I S$ & $000^{i} \angle 8 Z^{2} \varsigma S I S$ & 05 & 000'0EI'6ITS & $20 \mathrm{I}-\mathrm{ZV}$ \\
\hline $0 \$$ & $000^{\circ} \angle 6 S^{\circ} 69 \$$ & 0 s & $000^{\prime} 8 \varepsilon L^{\prime} 001 \mathrm{TS}$ & OS & $000^{6} \angle 65^{6} 69 \mathrm{~S}$ & $\mathrm{I} 0 \mathrm{I}-\mathrm{ZV}$ \\
\hline 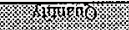 & 10ump & 943 & Syinung & 64u⿰n丨 & \% & \\
\hline 2017 \% & 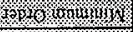 & $1016 \%$ op & 3.0. & 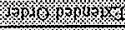 & yor & \\
\hline \% & Tour & \% & Houk & 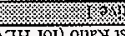 & 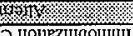 & \\
\hline
\end{tabular}

\begin{tabular}{|c|c|c|c|c|c|c|}
\hline $00 L^{\prime} 00 L^{\prime} I Z I S$ & $000^{\circ} 520^{\circ} 9525$ & $008^{\circ} \varepsilon \varepsilon S^{\prime} 90 z S$ & $000^{\circ} 920^{\circ} 952 S$ & OSZ $レ \varepsilon Z C 8 S$ & $000^{\circ} S 20952 S$ & [E10I \\
\hline $00 L^{6} 00 L^{\circ} I Z I S$ & $000^{\prime} 862^{i} \angle 9 S$ & OSE'TOt't6IS & $0 \mathrm{~s}$ & $0 \varsigma Z^{6} \downarrow \varepsilon Z^{\circ} \varepsilon 8 \$$ & $000^{\prime} 862^{\prime} \angle 9 S$ & $20 \mathrm{r}-\lambda \mathrm{V}$ \\
\hline $0 \mathrm{~s}$ & $000^{\circ} 08$ I'6IIS $^{\prime}$ & $0 S t^{6} Z E I^{\circ} z I S$ & $000^{\prime} \angle 8 Z^{\prime}$ SSIS & $O S$ & $000^{\circ} 08 I^{\circ} 611 \mathrm{~S}$ & $20 I-2 \mathrm{~V}$ \\
\hline $0 S$ & $000^{\circ} \angle 6 S^{\prime} 69 \$$ & 05 & $000^{\circ} 8 E L^{\circ} 001 S$ & OS & $000^{\circ} \angle 6 S^{\prime} 69 S$ & $10 \mathrm{I}-\mathrm{ZV}$ \\
\hline 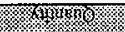 & (6) & 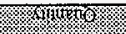 & 46 & 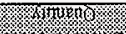 & W & \\
\hline 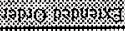 & 4. & 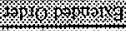 & Sojo & 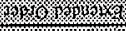 & 10. & \\
\hline (6) & 14k & $3 \%$ & $y=$ & 權药 & 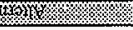 & \\
\hline
\end{tabular}

\begin{tabular}{|c|c|c|c|c|c|c|}
\hline $00 t^{4} 8 \mathrm{LI}^{\circ} 80 \mathrm{TS}$ & $000^{\circ} \$ 20^{\circ} 952 \$$ & $009^{\circ} \$ 8 S^{\prime} \varepsilon 8 \mathrm{IS}$ & $000^{\circ} s 20^{\circ} 9 S 2 S$ & $000^{\circ} 986^{\circ} \varepsilon L S$ & $000^{\circ} \mathrm{szO}^{\circ} 9 \mathrm{scs}$ & [E]OJ \\
\hline $000^{\circ} 8 L^{\prime} 80 I^{\prime}$ & $000^{\circ} 86 Z^{6} \angle 9 S$ & $00 Z^{6}$ I08 ZLIS & OS & $000^{\prime} 986^{4} \varepsilon L S$ & $000^{\circ} 86 Z^{2} \angle 9 S$ & $201-X V$ \\
\hline $0 S$ & $000^{\prime} 0$ EI'6IIS $^{\circ}$ & $005^{6}+8 L^{\circ} 0$ IS & $000^{6} \mathrm{LSZ}^{6}$ SSIS & $0 S$ & $000^{\circ} 0 E I^{\prime} 6115$ & $20 \mathrm{I}-\mathrm{ZV}$ \\
\hline $0 S$ & $000^{2} \angle 6 S^{\prime} 69 S$ & os & $000^{c} 8 \varepsilon L^{\prime} 00 \mathrm{I} \$$ & $0 S$ & $000^{\circ} \angle 6 S^{6} 69 S$ & IOI-Z \\
\hline 1961) & 1010) & 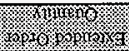 & 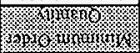 & 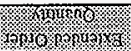 & 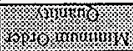 & \\
\hline 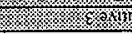 & 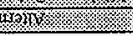 & Wrin & yüs & 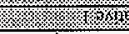 & $\sin _{11} / \mathrm{k} / \mathrm{k}$ & \\
\hline
\end{tabular}

\begin{tabular}{|c|c|c|c|c|c|c|}
\hline $00 I^{\prime} 959^{\prime}+65$ & $000^{\circ} 520^{\circ} 9 S 25$ & $00 t^{\prime} \angle 89^{\prime} 09$ IS & $000^{\circ} \$ 20^{\circ} 9 s 2 s$ & $0 S L^{\circ} L E L t 9 S$ & $000^{\circ} 5209525$ & 1E3OI \\
\hline $00 I^{\circ} 959^{\circ}+65$ & $000^{\circ} 86 C^{\prime} \mathrm{LOS}$ & OSO'IOZ'ISIS & os & $0 S L^{\prime} \angle E L^{\circ} \square 9 S$ & $000^{\circ} 86 Z^{\circ} \angle 9 S$ & $201-X V$ \\
\hline $0 S$ & $000^{\circ} 0$ EI $^{\prime} 6 \mathrm{IIS}$ & $0 S \varepsilon^{\prime} 9 \varepsilon t^{\prime} 6 S$ & $000^{\circ} \angle 8 Z^{\prime} S S I S$ & $0 S$ & 000.0 II $^{\circ}$ IIS & $20 \mathrm{~T}^{-2 \mathrm{ZV}}$ \\
\hline $0 \$$ & $000^{2} \angle 6 S^{\prime} 69 \mathrm{~S}$ & 05 & $000^{\prime} 8 E L^{\prime} 001 S$ & OS & $000^{\circ} \angle 6 S^{\prime} 69 S$ & IOI-ZV \\
\hline 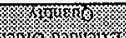 & (x) & (x) & 15urno & 350 & 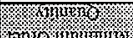 & \\
\hline 3rop & $\frac{1010}{154} \mathrm{~K}$ & $80 \%$ & Winur & 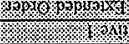 & moln & \\
\hline
\end{tabular}

\begin{tabular}{|c|c|c|c|c|c|c|}
\hline $008^{\circ}$ EEI I 8 S & $000^{\circ} s 20^{\circ} 9 \leq 2 S$ & O0Z'689'LEIS & $000^{\circ} 520^{\circ} 9525$ & $00 S^{4} 68 t^{\circ}$ SSS & $000^{\circ} 520^{\circ} 9525$ & IE]OI \\
\hline $008^{\prime} \varepsilon E I^{\prime} 18 S$ & $000^{\circ} 86 Z^{\circ} \mathrm{LgS}$ & $006^{\circ} 009^{6} 62$ IS & $0 S$ & $00 s^{2} 68 t^{\prime} s s s$ & $000^{2} 86 Z^{4} \angle 9 \$$ & $20 \tau-\lambda V$ \\
\hline $0 S$ & 000'0EI'6IIS & $00 \varepsilon^{\prime} 880^{\circ} 8 \$$ & $000^{\prime} \angle 8 z^{2}$ SSIS & $0 s$ & $000^{\circ} 0 \varepsilon I^{\prime} 6$ IS & $20 \mathrm{I}-\mathrm{ZV}$ \\
\hline $0 s$ & $000^{\circ} \angle 6 S^{\circ} 695$ & $0 \$$ & $000^{\prime} 8 E L^{\circ} 00$ IS & $0 \$$ & $000^{\circ} \angle 6 S^{\prime} 69 S$ & $10[-Z Y$ \\
\hline 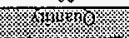 & KKMU⿴囗十 & (96) & (6. & \% & 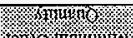 & \\
\hline $8 \% 00 \% 10 \%$ & $s p 0,113(140$ & $1411 \% \% \%$ & 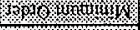 & \% & $92 \%$ Y Y W & \\
\hline 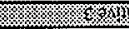 & 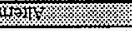 & (2) & How1 & 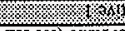 & 143 & \\
\hline
\end{tabular}

$$
\begin{aligned}
& \text { (9 jo } \rightarrow 7204 \mathrm{~S}) \\
& \text {. ( }
\end{aligned}
$$

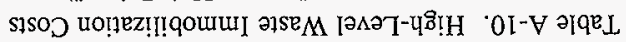

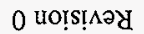

$$
\begin{aligned}
& \text { EOO-VDV-XML-GS-HNH }
\end{aligned}
$$


HNF-SD-TWR-AGA-003

Revision 0

Table A-10. High-Level Waste Immobilization Costs

(Based on BNFL, Inc. "Target-Unit-Prices").

(Sheet 5 of 6 )

\begin{tabular}{|c|c|c|c|c|c|c|}
\hline & \multicolumn{4}{|c|}{ Immobilization Cost Ratio (for HLW and LAW Services) $=$} & \multicolumn{2}{|l|}{0.6} \\
\hline & \multicolumn{2}{|c|}{ X } & \multicolumn{2}{|c|}{ 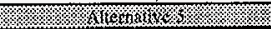 } & \multicolumn{2}{|c|}{ 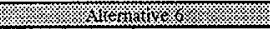 } \\
\hline & Whining & 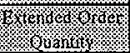 & 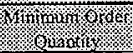 & Whor & Whinghor & Krow \\
\hline$A Z-101$ & $\$ 100,738,000$ & $\$ 0$ & $\$ 69,597,000$ & \$o & $\$ 69,597,000$ & $\$ 0$ \\
\hline $\mathrm{AZ}-102$ & $\$ 119,130,000$ & $\$ 0$ & $\$ 168,767,500$ & $\$ 0$ & $\$ 168,767,500$ & So \\
\hline$A Y-102$ & $\$ 36,157,000$ & $\$ 74,174,100$ & $\$ 17,660,500$ & $\$ 85,272,000$ & $\$ 17,660,500$ & $\$ 110,916,300$ \\
\hline Total & $\$ 256,025,000$ & $\$ 74,174,100$ & $\$ 256,025,000$ & $\$ 85,272.000$ & $\$ 256,025,000$ & $\$ 110,916,300$ \\
\hline
\end{tabular}

\begin{tabular}{|c|c|c|c|c|c|c|}
\hline & \multicolumn{4}{|c|}{ Immobilization Cost Ratio (for HLW and LAW Services) $=$} & \multicolumn{2}{|l|}{0.7} \\
\hline & \multicolumn{2}{|c|}{ W. } & \multicolumn{2}{|c|}{ 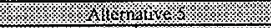 } & \multicolumn{2}{|c|}{ 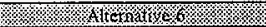 } \\
\hline & 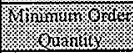 & 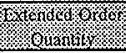 & 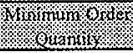 & K & 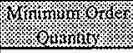 & W \\
\hline AZ-101 & $\$ 100,738,000$ & So & $\$ 69,597,000$ & \$0 & $\$ 69,597,000$ & $\$ 0$ \\
\hline $\mathrm{AZ}-102$ & $\$ 119,130,000$ & So & $\$ 168,767,500$ & $\$ 0$ & $\$ 168,767,500$ & so \\
\hline $\mathrm{AY}-102$ & $\$ 36,157,000$ & $\$ 86,536,450$ & $\$ 17,660,500$ & $\$ 99,484,000$ & $\$ 17,660,500$ & $\$ 129,402,350$ \\
\hline Total & $\$ 256,025,000$ & $\$ 86,536.450$ & $\$ 256.025 .000$ & $599,484,000$ & $\$ 256,025,000$ & $\$ 129,402,350$ \\
\hline
\end{tabular}

Immobilization Cost Ratio (for HLW and LAW Services) =

0.8

\begin{tabular}{|c|c|c|c|c|c|c|}
\hline & \multicolumn{4}{|c|}{ Immobilization Cost Ratio (for HLW and LAW Services) $=$} & \multicolumn{2}{|l|}{0.8} \\
\hline & s. & 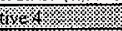 & 1 & E⿰亻 & Aterin & 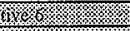 \\
\hline & Whining & Wrow & HNM Now & 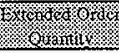 & Why & Krick \\
\hline AZ-101 & $\$ 100,738,000$ & \$0 & $\$ 69,597,000$ & $\$ 0$ & $\$ 69,597,000$ & so \\
\hline $\mathrm{AZ}-102$ & $\$ 119,130,000$ & $\$ 0$ & $\$ 168,767,500$ & so & $\$ 168,767,500$ & \$o \\
\hline$A Y-102$ & $\$ 36,157,000$ & $\$ 98,898,800$ & $\$ 17,660,500$ & $\$ 113,696,000$ & $\$ 17,660,500$ & $\$ 147,888,400$ \\
\hline Total & $\$ 256,025,000$ & $\$ 98,898,800$ & $\$ 256.025,000$ & $\$ 113,696,000$ & $\$ 256,025,000$ & $\$ 147,888,400$ \\
\hline
\end{tabular}

\begin{tabular}{|c|c|c|c|c|c|c|}
\hline & \multicolumn{4}{|c|}{ Immobilization Cost Ratio (for HLW and LAW Services) $=$} & \multicolumn{2}{|l|}{0.9} \\
\hline & \multicolumn{2}{|c|}{ 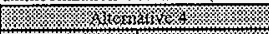 } & \multicolumn{2}{|c|}{ \% } & \multicolumn{2}{|c|}{ \% } \\
\hline & W. & 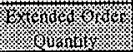 & W/10 & 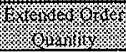 & Wyin & Mrom \\
\hline $\mathrm{AZ}-101$ & $\$ 100,738,000$ & So & $\$ 69,597,000$ & $\$ 0$ & $\$ 69,597,000$ & $\$ 0$ \\
\hline AZ-102 & $\$ 119,130,000$ & $\$ 0$ & $\$ 168,767,500$ & $\$ 0$ & $\$ 168,767,500$ & $\$ 0$ \\
\hline $\mathrm{AY}-102$ & $\$ 36,157,000$ & $\$ 111,261,150$ & $\$ 17,660,500$ & $\$ 127,908,000$ & $\$ 17,660,500$ & $\$ 166,374,450$ \\
\hline Total & $\$ 256.025,000$ & $\$ 111.261,150$ & $\$ 256,025,000$ & $\$ 127,908,000$ & $\$ 256,025,000$ & $\$ 166,374,450$ \\
\hline
\end{tabular}

\begin{tabular}{|c|c|c|c|c|c|c|}
\hline & \multicolumn{4}{|c|}{ Immobilization Cost Ratio (for HLW and LAW Services) = } & \multicolumn{2}{|l|}{1.0} \\
\hline & \multicolumn{2}{|c|}{ W } & \multicolumn{2}{|c|}{ \% } & \multicolumn{2}{|c|}{ 18.6\% } \\
\hline & Whinumord & 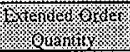 & 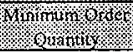 & 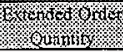 & Wyin mox & Wydrow \\
\hline $\mathrm{AZ}-101$ & $\$ 100,738,000$ & \$0 & $\$ 69,597,000$ & $\$ 0$ & $\$ 69,597,000$ & so \\
\hline $\mathrm{AZ}-102$ & $\$ 119,130,000$ & so & $\$ 168.767,500$ & so & $\$ 168,767,500$ & \$0 \\
\hline AY-102 & $\$ 36,157,000$ & $\$ 123,623,500$ & $\$ 17,660,500$ & $\$ 142,120,000$ & $\$ 17,660,500$ & $\$ 184,850,500$ \\
\hline Total & $\$ 256.025 .000$ & $\$ 123.623 .500$ & $\$ 256.025,000$ & $\$ 142,120.000$ & $\$ 256.025,000$ & $\$ 184,860,500$ \\
\hline
\end{tabular}


HNF-SD-TWR-AGA-003

Revision 0

Table A-10. High-Level Waste Immobilization Costs

(Based on BNFL, Inc. "Target-Unit-Prices").

(Sheet 6 of 6 )

\begin{tabular}{|c|c|c|c|c|}
\hline & \multicolumn{4}{|c|}{ Immobilization Cost Ratio (for HLW and LAW Services) $=$} \\
\hline & \multicolumn{2}{|c|}{ W } & \multicolumn{2}{|c|}{ 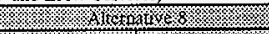 } \\
\hline & 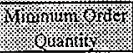 & Wowororo & 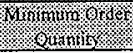 & 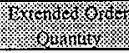 \\
\hline AZ-101 & $\$ 100,738,000$ & so & $\$ 100,738.000$ & $\$ 0$ \\
\hline AZ-102 & $\$ 119,130,000$ & $\$ 0$ & $\$ 155,287,000$ & $\$ 8,088,300$ \\
\hline $\mathrm{AY}-102$ & $\$ 36,157,000$ & $\$ 99,818,400$ & so & $\$ 103,956,600$ \\
\hline Total & $\$ 256.025,000$ & $\$ 99,818.400$ & $\$ 256.025 .000$ & $\$ 112,044,900$ \\
\hline
\end{tabular}

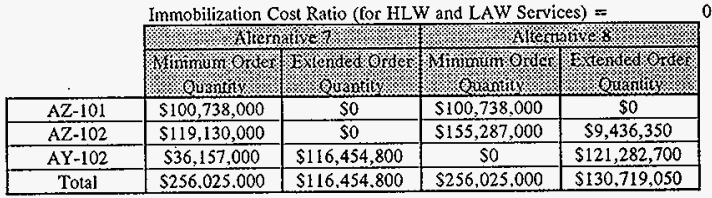

\begin{tabular}{|c|c|c|c|c|}
\hline & \multicolumn{4}{|c|}{ Immobilization Cost Ratio (for HLW and LAW Services) $=$} \\
\hline & \multicolumn{2}{|c|}{ W } & \multicolumn{2}{|c|}{ 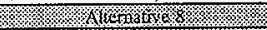 } \\
\hline & 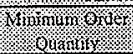 & Kxy & 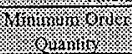 & Now \\
\hline $\mathrm{AZ}-101$ & $\$ 100,738,000$ & So & $\$ 100,738,000$ & \$0 \\
\hline $\mathrm{AZ}-102$ & $\$ 119,130,000$ & $\$ 0$ & $\$ 155,287,000$ & $\$ 10,784,400$ \\
\hline $\mathrm{AY}-102$ & $\$ 36,157,000$ & $\$ 133,091,200$ & $\$ 0$ & $\$ 138,608,800$ \\
\hline Total & $\$ 256.025,000$ & $\$ 133.091 .200$ & $\$ 256,025,000$ & $\$ 149,393,200$ \\
\hline
\end{tabular}

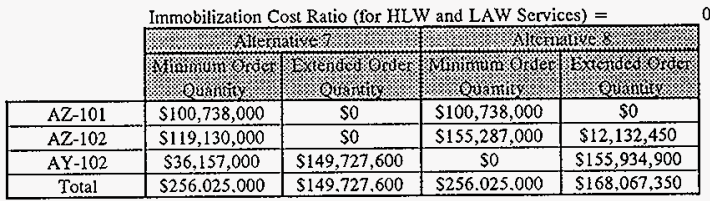

\begin{tabular}{|c|c|c|c|c|}
\hline & \multicolumn{4}{|c|}{ Immobilization Cost Ratio (for HLW and LAW Services) $=$} \\
\hline & \multicolumn{2}{|c|}{ 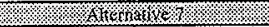 } & \multicolumn{2}{|c|}{ 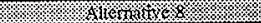 } \\
\hline & 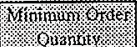 & W & Whinungro & Wroworow \\
\hline $\mathrm{AZ}-101$ & $\$ 100.738,000$ & so & $\$ 100.738 .000$ & $\$ 0$ \\
\hline AZ- 102 & $\$ 119.130 .000$ & so & $\$ 155,287,000$ & $\$ 13,480,500$ \\
\hline $\mathrm{AY}-102$ & $\$ 36.157,000$ & $\$ 166.364,000$ & So & $\$ 173,261,000$ \\
\hline Total & $\$ 256.025 .000$ & $\$ 166.364 .000$ & $\$ 256.025 .000$ & $\$ 186.741,500$ \\
\hline
\end{tabular}


HNF-SD-TWR-AGA-003

Revision 0

Table A-11. High-Level Waste Immobilization Costs

(Based on LMAES "Target-Unit-Prices").

(Sheet 1 of 6)

\begin{tabular}{|c|c|c|c|c|c|c|}
\hline & \multicolumn{4}{|c|}{ Immobilization Cost Ratio (for HLW and LAW Services) $=$} & \multicolumn{2}{|l|}{0.0} \\
\hline & \multicolumn{2}{|c|}{ \& 6 . } & \multicolumn{2}{|c|}{ 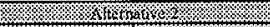 } & \multicolumn{2}{|c|}{ K } \\
\hline & Wfinom & 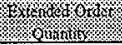 & 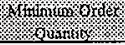 & 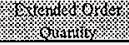 & 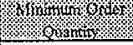 & 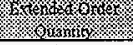 \\
\hline $\mathrm{AZ}-101$ & $\$ 483,469,380$ & $\$ 0$ & $\$ 699,796,520$ & so & $\$ 483,469,380$ & $\$ 0$ \\
\hline $\mathrm{AZ}-102$ & $\$ 827,560,200$ & $\$ 0$ & $\$ 1,078,731,980$ & \$o & $\$ 827,560,200$ & $\$ 0$ \\
\hline $\mathrm{AX}-102$ & $\$ 467,498,920$ & so & $\$ 0$ & $\$ 0$ & $\$ 467,498,920$ & $\$ 0$ \\
\hline Total & $\$ 1,778,528,500$ & so & $\$ 1,778,528,500$ & \$0 & $\$ 1.778 .528 .500$ & so \\
\hline
\end{tabular}

\begin{tabular}{|c|c|c|c|c|c|c|}
\hline & \multicolumn{4}{|c|}{ Immobilization Cost Ratio (for HLW and LAW Services) = } & \multicolumn{2}{|l|}{0.1} \\
\hline & \multicolumn{2}{|c|}{ 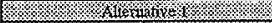 } & \multicolumn{2}{|c|}{ WHitratike } & \multicolumn{2}{|c|}{ 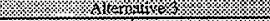 } \\
\hline & 1. & W & Kowhor & Whow & Whinimordo & 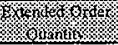 \\
\hline$A Z-101$ & $\$ 483,469,380$ & 50 & $\$ 699,796,520$ & $\$ 0$ & $\$ 483,469,380$ & \$o \\
\hline AZ-102 & $\$ 827,560,200$ & so & $\$ 1,078,731,980$ & $\$ 9,364,497$ & $\$ 827,560,200$ & $\$ 0$ \\
\hline AY -102 & $\$ 467,498,920$ & $\$ 64,244,805$ & $\$ 0$ & $\$ 150,049,731$ & $\$ 467,498,920$ & $\$ 93,935,342$ \\
\hline Total & $\$ 1,778,528,500$ & $\$ 64,244,805$ & $\$ 1,778,528,500$ & $\$ 159.414,228$ & $\$ 1.778 .528 .500$ & $\$ 93,935,342$ \\
\hline
\end{tabular}

tio (for HLW and LAW Services) =

0.2

\begin{tabular}{|c|c|c|c|c|c|c|}
\hline & \multicolumn{4}{|c|}{ Immobilization Cost Ratio (for HLW and LAW Services) = } & \multicolumn{2}{|l|}{0.2} \\
\hline & \multicolumn{2}{|c|}{ 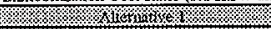 } & \multicolumn{2}{|c|}{ / } & \multicolumn{2}{|c|}{ F/ } \\
\hline & Whoring & Wown & 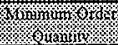 & 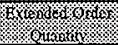 & Mrow & 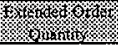 \\
\hline AZ-101 & $\$ 483,469,380$ & $\$ 0$ & $\$ 699,796,520$ & $\frac{\$ 0}{50}$ & $\$ 483,469.380$ & $\$ 0$ \\
\hline $\mathrm{Az}-102$ & $\$ 827,560,200$ & $\$ 0$ & $\$ 1,078,731,980$ & $\$ 18,728,994$ & $\$ 827,560,200$ & $\$ 0$ \\
\hline AY -102 & $\$ 467,498,920$ & $\$ 128,489,610$ & $\$ 0$ & $\$ 300,099,462$ & $\$ 467,498,920$ & $\$ 187,870,684$ \\
\hline Total & $\$ 1,778,528,500$ & $\$ 128,489,610$ & $\$ 1.778,528,500$ & $\$ 318.828 .456$ & $\$ 1.778 .528 .500$ & $\$ 187,870,684$ \\
\hline
\end{tabular}

\begin{tabular}{|c|c|c|c|c|c|c|}
\hline \multirow{3}{*}{. } & \multicolumn{4}{|c|}{ Immobilization Cost Ratio (for HLW and LAW Services) $=$} & \multicolumn{2}{|l|}{0.3} \\
\hline & \multicolumn{2}{|c|}{ \% } & \multicolumn{2}{|c|}{ 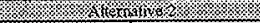 } & \multicolumn{2}{|c|}{ 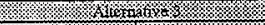 } \\
\hline & Whing & yourow & 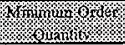 & \% & 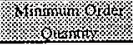 & W \\
\hline AZ-101 & $\$ 483,469,380$ & \$0 & $\$ 699,796,520$ & \$o & $\$ 483,469,380$ & $\$ 0$ \\
\hline$\overline{\mathrm{AZ}-102}$ & $\$ 827,560,200$ & \$o & $\$ 1,078,731,980$ & $\$ 28,093,491$ & $\$ 827,560,200$ & $\$ 0$ \\
\hline AY-102 & $\$ 467,498,920$ & $\$ 192.734,415$ & $\$ 0$ & $\$ 450,149,193$ & $\$ 467,498,920$ & $\$ 281,806,026$ \\
\hline Total & $\$ 1,778, \$ 28,500$ & $\$ 192.734,415$ & $\$ 1.778 .528 .500$ & $\$ 478.242 .684$ & $\$ 1,778,528,500$ & $\$ 281,806,026$ \\
\hline
\end{tabular}

\begin{tabular}{|c|c|c|c|c|c|c|}
\hline & \multicolumn{4}{|c|}{ Immobilization Cost Ratio (for HLW and LAW Services) $=$} & \multicolumn{2}{|c|}{0.4} \\
\hline & \multicolumn{2}{|c|}{ \% \% } & \multicolumn{2}{|c|}{ \% } & \multicolumn{2}{|c|}{ 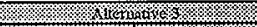 } \\
\hline & Hin & Hor & Woration & \% & Wink Ming & Krown \\
\hline AZ-10I & $\$ 483,469,380$ & so & $\$ 699,796,520$ & \$o & $\$ 483,469,380$ & $\$ 0$ \\
\hline $\mathrm{AZ}-102$ & $\$ 827,560,200$ & $\$ 0$ & $\$ 1,078,731,980$ & $\$ 37,457,988$ & $\$ 827,560,200$ & $\$ 0$ \\
\hline$A Y-102$ & $\$ 467,498,920$ & $\$ 256,979,220$ & $\$ 0$ & $\$ 600,198,924$ & $\$ 467,498,920$ & $\$ 375,741,368$ \\
\hline Total & $\$ 1,778.528,500$ & $\$ 256.979 .220$ & $\$ 1,778.528 .500$ & $\$ 637.656,912$ & $\$ 1.778 .528,500$ & $\$ 375,741,368$ \\
\hline
\end{tabular}

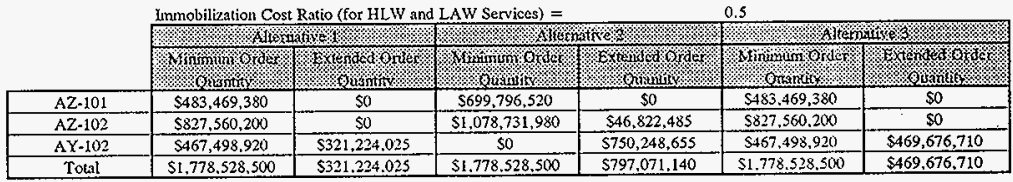


HNF-SD-TWR-AGA-003

\section{Revision 0}

\section{Table A-11. High-Level Waste Immobilization Costs \\ (Based on LMAES "Target-Unit-Prices"). \\ (Sheet 2 of 6)}

\begin{tabular}{|c|c|c|c|c|c|c|}
\hline & \multicolumn{4}{|c|}{ Immobilization Cost Ratio (for HLW and LAW Services) $=$} & \multicolumn{2}{|c|}{0.0} \\
\hline & \multicolumn{2}{|c|}{ 16. } & \multicolumn{2}{|c|}{ King } & \multicolumn{2}{|c|}{ 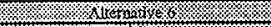 } \\
\hline & Whom & W & Whom & 6) & 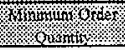 & 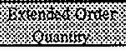 \\
\hline $\mathrm{AZ}-101$ & $\$ 699,796,520$ & so & $\$ 483,469,380$ & so & $\$ 483.469 .380$ & $\$ 0$ \\
\hline$A Z-102$ & $\$ 827,560,200$ & so & $\$ 1,172,376,950$ & $\$ 0$ & $\$ 1,172,376,950$ & $\$ 0$ \\
\hline AY-102 & $\$ 251,171,780$ & 50 & $\$ 122,682,170$ & $\$ 0$ & $\$ 122,682,170$ & \$o \\
\hline Total & $\$ 1,778,528,500$ & So & $\$ 1.778,528.500$ & $\$ 0$ & $\$ 1,778,528,500$ & so \\
\hline
\end{tabular}

\begin{tabular}{|c|c|c|c|c|c|c|}
\hline & \multicolumn{4}{|c|}{ Immobilization Cost Ratio (for HLW and LAW Services) $=$} & \multicolumn{2}{|c|}{0.1} \\
\hline & \multicolumn{2}{|c|}{ \% \% \% } & \multicolumn{2}{|c|}{ \%.\% 1 H } & \multicolumn{2}{|c|}{ \% } \\
\hline & W & 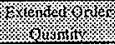 & Wringinging & 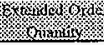 & Whomolom & Wyid \\
\hline$A Z-101$ & $\$ 699,796, \$ 20$ & $\$ 0$ & $\$ 483,469,380$ & so. & $\$ 483,469,380$ & $\$ 0$ \\
\hline AZ-102 & $\$ 827,560,200$ & $\$ 0$ & $\$ 1,172,376,950$ & so & $\$ 1,172,376,950$ & so \\
\hline $\mathrm{AY}-102$ & $\$ 251,171,780$ & $\$ 85,877,519$ & $\$ 122,682,170$ & $\$ 98,726,480$ & $\$ 122,682,170$ & $\$ 128,417,017$ \\
\hline Total & $\$ 1,778,528,500$ & $\$ 85,877,519$ & $\$ 1,778,528,500$ & $\$ 98,726,480$ & $\$ 1.778 .528 .500$ & $\$ 128,417,017$ \\
\hline
\end{tabular}

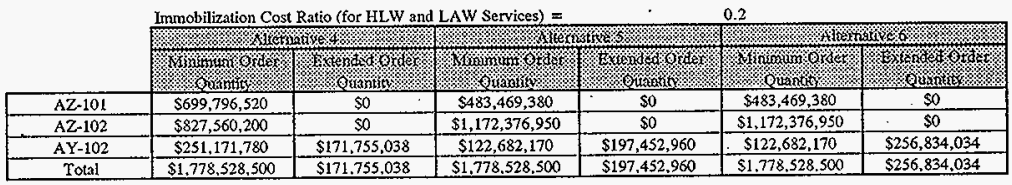

\begin{tabular}{|c|c|c|c|c|c|c|}
\hline & \multicolumn{4}{|c|}{ Immobjlization Cost Ratio (for HLW and LAW Services) $=$} & \multicolumn{2}{|c|}{0.3} \\
\hline & \multicolumn{2}{|c|}{ 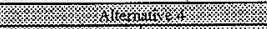 } & \multicolumn{2}{|c|}{ 雨 \% } & \multicolumn{2}{|c|}{ W.\%. } \\
\hline & W & Ousnolls & Hown & 3. & Homords & Grow \\
\hline AZ-101 & $\$ 699,796,520$ & so & $\$ 483,469,380$ & so & $\$ 483,469,380$ & $\$ 0$ \\
\hline$\overline{A Z-102}$ & $\$ 827,560,200$ & $\$ 0$ & $\$ 1,172,376,950$ & so & $\$ 1,172,376, \$ 50$ & $\$ 0$ \\
\hline AY-102 & $\$ 251,171,780$ & $\$ 257,632,557$ & $\$ 122,682,170$ & $\$ 296,179,440$ & $\$ 122,682,170$ & $\$ 385,251,051$ \\
\hline Total & $\$ 1,778,528,500$ & $\$ 257,632,557$ & $\$ 1,778,528,500$ & $\$ 296.179 .440$ & $\$ 1,778,528,500$ & $\$ 385,251,051$ \\
\hline
\end{tabular}

\begin{tabular}{|c|c|c|c|c|c|c|}
\hline & \multicolumn{4}{|c|}{ Immobilization Cost Ratio (for HLW and LAW Services) $=$} & \multicolumn{2}{|c|}{0.4} \\
\hline & \% & א. & 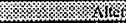 & (1) & SUES & K \\
\hline & Whung & KKo & y.t. & Kown & Whinin & 3row \\
\hline AZ-101 & $\$ 699,796,520$ & \$o & $\$ 483,469,380$ & so & $\$ 483,469,380$ & so \\
\hline $\mathrm{AZ}-102$ & $\$ 827,560,200$ & so & $\$ 1,172,376,950$ & \$o & $\$ 1,172,376,950$ & $\$ 0$ \\
\hline$A Y-102$ & $\$ 251.171 .780$ & $\$ 343,510,076$ & $\$ 122,682,170$ & $\$ 394,905,920$ & $\$ 122,682,170$ & $\$ \$ 13,668,068$ \\
\hline Total & $\$ 1.778,528.500$ & $\$ 343,510.076$ & $\$ 1.778 .528 .500$ & $\$ 394,905,920$ & $\$ 1.778,528,500$ & $\$ \$ 13,668,068$ \\
\hline
\end{tabular}

\begin{tabular}{|c|c|c|c|c|c|c|}
\hline & \multicolumn{4}{|c|}{ Inmobilization Cost Ratio (for HLW and LAW Services) $=$} & \multicolumn{2}{|l|}{0.5} \\
\hline & V. & $1 \% 6 \%$ & Q & Hos & $\% 8$ & 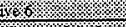 \\
\hline & WWing & $6 \%$ & 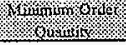 & Kow & Whondoring & 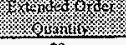 \\
\hline AZ-101 & $\$ 699,796,520$ & so & $\$ 483,469,380$ & so & $\$ 483,469,380$ & so \\
\hline AZ-102 & $\$ 827,560,200$ & So & $\$ 1,172,376,950$ & 50 & $\$ 1,172,376,950$ & so \\
\hline$A Y-102$ & $\$ 251,171.780$ & $\$ 429.387 .595$ & $\$ 122,682,170$ & $\$ 493,632,400$ & 5122.682 .170 & $\$ 642,085,085$ \\
\hline Total & $\$ 1.778 .528 .500$ & 5429.387 .595 & $\$ 1.778 .528 .500$ & $\$ 493,632.400$ & 51.778 .528 .500 & $\$ 642,085,085$ \\
\hline
\end{tabular}


HNF-SD-TWR-AGA-003

\section{Revision 0}

Table A-11. High-Level Waste Immobilization Costs

(Based on LMAES "Target-Unit-Prices").

(Sheet 3 of 6)

\begin{tabular}{|c|c|c|c|c|}
\hline & \multicolumn{4}{|c|}{ Immobilization Cost Ratio (for HLW and LAW Services) $=$} \\
\hline & \multicolumn{2}{|c|}{ 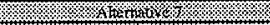 } & \multicolumn{2}{|c|}{ W Wr. } \\
\hline & W M & Wroworid & Whon & How \\
\hline$A Z-101$ & $\$ 699,796,520$ & $\$ 0$ & $\$ 699,796,520$ & $\$ 0$ \\
\hline AZ-102 & $\$ 827,560,200$ & $\$ 0$ & $\$ 1,078,731,980$ & $\$ 0$ \\
\hline$A Y-102$ & $\$ 251,171,780$ & $\$ 0$ & so & $\$ 0$ \\
\hline Total & $\$ 1.778 .528,500$ & $\$ 0$ & $\$ 1,778,528,500$ & so \\
\hline
\end{tabular}

\begin{tabular}{|c|c|c|c|c|}
\hline & \multicolumn{4}{|c|}{ Inmobilization Cost Ratio (for HLW and LAW Services) = } \\
\hline & Yrow & \% W & Wininin & 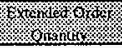 \\
\hline$A Z-101$ & $\$ 699.796 .520$ & $\$ 0$ & $\$ 699,796,520$ & so \\
\hline AZ-102 & $\$ 827,560,200$ & $\$ 0$ & $\$ 1,078,731,980$ & $\$ 9,364,497$ \\
\hline$A Y-102$ & $\$ 251,171,780$ & $\$ 115,568,056$ & $\$ 0$ & $\$ 120,359,194$ \\
\hline Total & $\$ 1,778,528,500$ & $\$ 115,568,056$ & $\$ 1,778,528,500$ & $\$ 129,723,691$ \\
\hline
\end{tabular}

\begin{tabular}{|c|c|c|c|c|}
\hline & \multicolumn{4}{|l|}{ Immobilization $\mathrm{Co}$} \\
\hline & \multicolumn{2}{|c|}{. } & \multicolumn{2}{|c|}{ 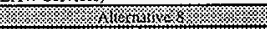 } \\
\hline & Mrnin & Whoro & 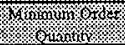 & 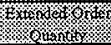 \\
\hline$A Z-101$ & $\$ 699,796,520$ & so & $\$ 699,796,520$ & $\$ 0$ \\
\hline AZ-102 & $\$ 827,560,200$ & $\$ 0$ & $\$ 1,078,731,980$ & $\$ 18,728,994$ \\
\hline$A X=102$ & $\$ 251,171,780$ & $\$ 231,136,112$ & 80 & $\$ 240,718,388$ \\
\hline Total & $\$ 1,778,528,500$ & $\$ 231,136,112$ & $\$ 1,778,528,500$ & $\$ 259.447,382$ \\
\hline
\end{tabular}

\begin{tabular}{|c|c|c|c|c|}
\hline & \multicolumn{4}{|c|}{ Inmobilization Cost Ratio (for HLW and LAW Services) $=$} \\
\hline & \multicolumn{2}{|c|}{ 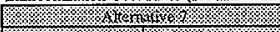 } & \multicolumn{2}{|c|}{ \% \% } \\
\hline & W & Wring & Mring & E Exy \\
\hline$A Z-101$ & $\$ 699,796,520$ & So & $\$ 699,796,520$ & so \\
\hline AZ-102 & $\$ 827,560,200$ & $\$ 0$ & $\$ 1,078,731,980$ & $\$ 28,093,491$ \\
\hline$A X-102$ & $\$ 251,171,780$ & $\$ 346,704,168$ & $\$ 0$ & $\$ 361,077,582$ \\
\hline Total & $\$ 1,778,528,500$ & $\$ 346,704,168$ & $\$ 1,778,528,500$ & $\$ 389,171,073$ \\
\hline
\end{tabular}

\begin{tabular}{|c|c|c|c|c|}
\hline & \multicolumn{4}{|c|}{ Immobilization Cost Ratio (for HLW and LAW Services) $=$} \\
\hline & \multicolumn{2}{|c|}{ 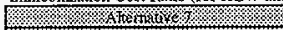 } & \multicolumn{2}{|c|}{ 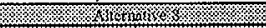 } \\
\hline & Wholing & 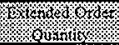 & 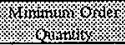 & 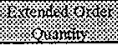 \\
\hline A2-101 & $\$ 699,796,520$ & $\$ 0$ & $\$ 699,796,520$ & so \\
\hline$A Z-102$ & $\$ 827,560,200$ & $\$ 0$ & $\$ 1,078,731,980$ & $\$ 37,457,988$ \\
\hline AY-102 & $\$ 251,171,780$ & $\$ 462,272,224$ & so & $\$ 481,436,776$ \\
\hline Total & $\$ 1.778,528,500$ & $\$ 462,272,224$ & $\$ 1.778,528,500$ & $\$ 518,894.764$ \\
\hline
\end{tabular}

\begin{tabular}{|c|c|c|c|c|}
\hline & \multicolumn{4}{|c|}{ Immobilization Cost Ratio (for HiLW and LAW Services) $=$} \\
\hline & \multicolumn{2}{|c|}{ 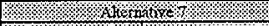 } & \multicolumn{2}{|c|}{ 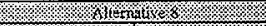 } \\
\hline & Whingining & 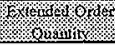 & 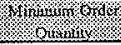 & Whong \\
\hline$A 2-101$ & $\$ 699,796,520$ & so & $\$ 699,796, \$ 20$ & \$o \\
\hline AZ-102 & $\$ 827,560,200$ & $\$ 0$ & $\$ 1,078,731,980$ & $\$ 46,822,485$ \\
\hline$A Y-102$ & $\$ 251,171,780$ & $\$ 577,840,280$ & \$o & $\$ 601,795,970$ \\
\hline Total & 51.778 .528 .500 & $\$ 577.840 .280$ & $\$ 1.778 .528 .500$ & $\$ 648,618,455$ \\
\hline
\end{tabular}


HNF-SD-TWR-AGA-003

Revision 0

Table A-11. High-Level Waste Immobilization Costs

(Based on LMAES "Target-Unit-Prices").

(Sheet 4 of 6)

\begin{tabular}{|c|c|c|c|c|c|c|}
\hline & \multicolumn{4}{|c|}{ Immobilization Cost Ratio (for HLW and LAW Services) = } & \multicolumn{2}{|c|}{0.6} \\
\hline & \multicolumn{2}{|c|}{ K } & \multicolumn{2}{|c|}{ 3.6. } & \multicolumn{2}{|c|}{ 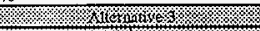 } \\
\hline & Whourom & 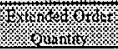 & Wh & work & 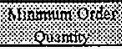 & \% \\
\hline $\mathrm{AZ}-101$ & $\$ 483,469,380$ & so & $\$ 699.796 .520$ & \$o & $\$ 483,469,380$ & so \\
\hline $\mathrm{AZ}-102$ & $\$ 827,560,200$ & so & $\$ 1,078,731,980$ & $\$ 56,186,982$ & $\$ 827,560,200$ & $\$ 0$ \\
\hline $\mathrm{AY}-102$ & $\$ 467,498,920$ & $\$ 385,468,830$ & 50 & $\$ 900,298,386$ & $\$ 467,498,920$ & $\$ 563,612,052$ \\
\hline Total & $\$ 1,778,528,500$ & $\$ 385,468.830$ & $\$ 1.778 .528 .500$ & $\$ 956.485 .368$ & $\$ 1,778,528,500$ & $\$ 563,612,052$ \\
\hline
\end{tabular}

\begin{tabular}{|c|c|c|c|c|c|c|}
\hline & \multicolumn{4}{|c|}{ Immobilization Cost Ratio (for HLW and LAW Services) $=$} & \multicolumn{2}{|l|}{0.7} \\
\hline & \multicolumn{2}{|c|}{ 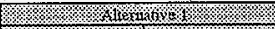 } & \multicolumn{2}{|c|}{ X } & \multicolumn{2}{|c|}{ 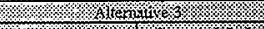 } \\
\hline & W & 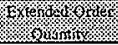 & Whouninin & Wornon & 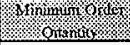 & Kownown \\
\hline AZ-101 & $\$ 483,469,380$ & $\$ 0$ & $\$ 699.796 .520$ & \$o & $\$ 483,469,380$ & $\$ 0$ \\
\hline $\mathrm{AZ}-102$ & $\$ 827,560,200$ & \$o & $\$ 1,078,731,980$ & $\$ 65,551,479$ & $5827,560,200$ & $\$ 0$ \\
\hline $\mathrm{AY}-102$ & $\$ 467,498,920$ & $\$ 449,713,635$ & $\$ 0$ & $\$ 1,050,348,117$ & $\$ 467,498,920$ & $\$ 657,547,394$ \\
\hline Total & $\$ 1.778,528.500$ & $\$ 449.713 .635$ & $\$ 1,778.528 .500$ & $\$ 1.115 .899 .596$ & $\$ 1.778,528,500$ & $\$ 657, \$ 47,394$ \\
\hline
\end{tabular}

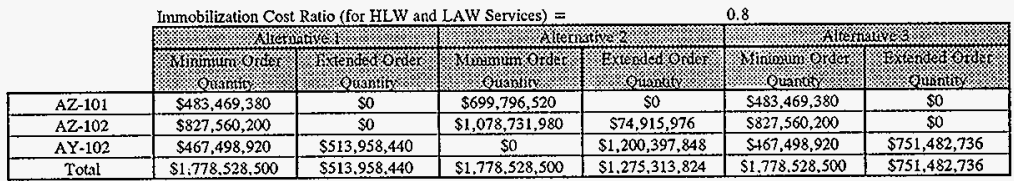

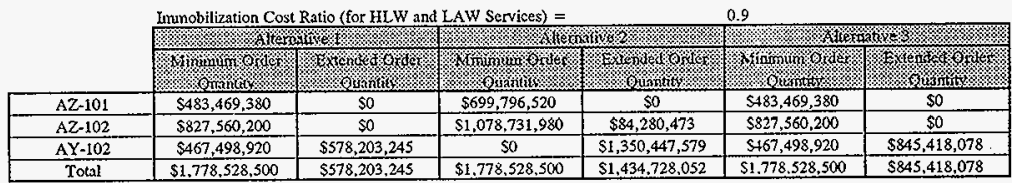

\begin{tabular}{|c|c|c|c|c|c|c|}
\hline & \multicolumn{4}{|c|}{ Immobilization Cost Ratio (for HLW and LAW Services) $=$} & \multicolumn{2}{|l|}{1.0} \\
\hline & \multicolumn{2}{|c|}{ 偠 } & \multicolumn{2}{|c|}{ 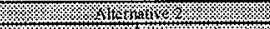 } & \multicolumn{2}{|c|}{ W } \\
\hline & Xinow & Woxtixk & 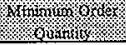 & Wrororos & 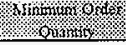 & 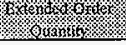 \\
\hline $\mathrm{AZ}-101$ & $\$ 483,469,380$ & so & $\$ 699,796,520$ & $\$ 0$ & $5483,469,380$ & so \\
\hline AZ-102 & $\$ 827,560,200$ & $\$ 0$ & $\$ 1,078,731,980$ & $\$ 93,644,970$ & $\$ 827,560,200$ & $\$ 0$ \\
\hline $\mathrm{AY}-102$ & $\$ 467,498,920$ & $\$ 64+2,448,050$ & $\$ 0$ & $\$ 1,500,497,310$ & $\$ 467.498 .920$ & $\$ 939,353,420$ \\
\hline Total & $\$ 1.778 .528 .500$ & $\$ 642,448,050$ & $\$ 1,778,528,500$ & $\$ 1.594,142.280$ & $\$ 1.778,528,500$ & $\$ 939.353 .420$ \\
\hline
\end{tabular}




\begin{tabular}{|c|c|c|c|c|c|c|}
\hline $0 L I^{2} O L I^{2}+8 Z^{2}$ IS & 00s'8ts' $8 L L^{\prime}$ is & $008^{\prime}+92^{2} \angle 865$ & $005.825 \% \angle L I S$ & $061^{\circ} S L L^{\circ} 8585$ & $00 S^{2} 825^{\circ} 8 L L^{\prime} T S$ & MOL \\
\hline $0 L I^{\prime} 0 L I^{2}+8 Z^{2} I S$ & $0 L I^{\circ} 289^{\circ} 2715$ & $008^{4}+9 \tau^{2} 2865$ & OLT'Z89'ZZIS & $061^{*} \$ L L^{\prime} 8585$ & 08LILI'ISZS & $20 I-\lambda Y$ \\
\hline $0 \$$ & OS6'9LE'ZLI'IS & OS & $056^{\circ} 9 L E^{\circ} T L I^{\prime}$ IS & $0 \mathrm{~S}$ & $00 z^{\prime} 09 s^{\prime} \angle 285$ & $201-Z \mathrm{Y}$ \\
\hline $0 s$ & $08 \varepsilon^{\prime} 69 t^{\prime} \varepsilon 8+5$ & OS & $08 \varepsilon^{\prime} 69 t^{\prime} \varepsilon 8 t S$ & os & $0 z 5^{\prime} 96 L^{\prime} 669 \mathrm{~S}$ & I0I-ZY \\
\hline 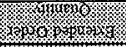 & 300 vontming & morno & \% & 3) & \% & \\
\hline \multicolumn{2}{|c|}{ 38\% \% } & \multicolumn{2}{|c|}{ 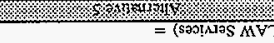 } & \multicolumn{2}{|c|}{ 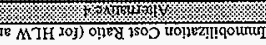 } & \\
\hline
\end{tabular}

\begin{tabular}{|c|c|c|c|c|c|c|}
\hline SSI'ESLSSI'IS & $005^{\prime} 8 \pi S^{\prime} 8 L L$ IS & $0 Z \varepsilon^{\circ} 8 \varepsilon S^{\circ} 888 S$ & $00 S^{2} 8 Z S^{4} 8 L L^{\prime}$ IS & $T \angle 9^{\circ} \angle 68^{\circ} Z \angle \angle S$ & $00 S^{\prime} 8 Z S^{\prime} 8 L L^{\prime} I S$ & mod \\
\hline ESI'ESL'SST' IS & OLT'Z\&9'ZZIS & $0 Z E^{\circ} 8 E S^{\prime} 888 S$ & $0 \angle T^{\prime} \mathrm{ZS9} 9^{\circ} \mathrm{ZTIS}$ & $\mathrm{I} \angle 9^{\circ} \angle 68^{\circ} \mathrm{ZLLS}$ & 08L'ILI'ISES & $20 \mathrm{l}-\mathrm{XY}$ \\
\hline $0 \$$ & OS6 ${ }^{\prime} 9 L E^{\prime} Z L I^{\prime}$ IS & os & $0 S 6^{\circ} 9 L \varepsilon^{\prime} T L I^{\prime} T \$$ & 05 & $00 z^{\prime} 095^{\prime} \angle 28 S$ & $20 \mathrm{~T}-\mathrm{ZV}$ \\
\hline os & $08 E^{\prime} 69688 \mathrm{ts}$ & $0 \$$ & $08 \varepsilon^{\prime} 695^{\prime} \varepsilon 8 t 5$ & OS & $0 \tau S^{\prime} 96 L^{2} 669 S$ & I0I-ZY \\
\hline Kry & 3. & 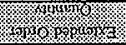 & 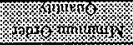 & \% & $\begin{array}{l}5 \\
6 \\
6\end{array}$ & \\
\hline \multicolumn{2}{|c|}{ \% } & \multicolumn{2}{|c|}{ 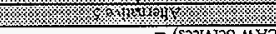 } & \multicolumn{2}{|c|}{ k } & \\
\hline
\end{tabular}

\begin{tabular}{|c|c|c|c|c|c|c|}
\hline 9ET $9 \varepsilon E$ L20' Is & $005^{\circ} 8 \pi 5^{\prime} 8 L L^{\prime} \mathrm{IS}$ & $08^{\circ} 118^{\circ} 68 L S$ & $00 S^{\prime} 8 Z S^{\prime} 8 L L$ IS & $251^{\circ} 020^{\prime} \angle 89 S$ & $005^{\prime} 825^{\prime} 8 L L^{\prime}$ TS & f버이 \\
\hline $98 I^{\prime} 9 \varepsilon \varepsilon^{\prime} \angle Z O^{\prime} I S$ & $0 I^{\circ} 289^{\circ} \mathrm{Z}$ IS & $0+8^{\prime} 118^{\circ} 68 \mathrm{LS}$ & $0 L L^{\prime} z 89^{\prime} z Z$ IS & $251^{\circ} 020^{\prime} \angle 89 S$ & $08 L^{\prime} T L I^{\prime} I S Z S$ & $20 \pi-\pi V$ \\
\hline os & $0 \$ 6^{\prime} 9 L \varepsilon^{\prime} Z L T^{\prime} T S$ & 05 & $0 S 6^{\prime} 9 L \varepsilon^{\prime} z L T^{\prime} I S$ & os & $00 Z^{*} 095^{\circ} \angle 28 S$ & $201-2 \mathrm{~V}$ \\
\hline os & $08 \varepsilon^{\prime} 69 b^{\circ} \varepsilon 8 t S$ & 05 & $08 \varepsilon^{4} 69 \nabla^{\prime} \varepsilon 8+5$ & 05 & $02 S^{2} 96 L^{\prime} 669 \$$ & $10 \pi-Z V$ \\
\hline 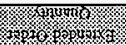 & \% & 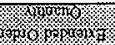 & 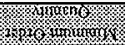 & (1) & \% & \\
\hline 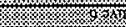 & mys & 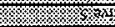 & S3M & 10 & $5 x$ & \\
\hline
\end{tabular}

\begin{tabular}{|c|c|c|c|c|c|c|}
\hline $6\left[1^{2} 616^{\circ} 868 \mathrm{~S}\right.$ & $005^{\circ} 825^{\circ} 8 L L^{\prime}$ IS & $09 \varepsilon^{\circ} \varsigma 80^{\prime} \mathrm{I} 69 \mathrm{~S}$ & $00 S^{\prime} 82 S^{\prime} 8 L L^{\prime} T S$ & $\left.\varepsilon \varepsilon 9^{\prime} Z \not\right]^{\prime} \mathrm{T} 09$ & $\cos ^{\prime} 8 Z S^{\prime} 8 L L^{\prime} 1 S$ & IMOI \\
\hline $61^{\circ} 616^{\circ} 868 \mathrm{~s}$ & $0 \angle I^{\prime} z 89^{\circ} z \mathrm{IS}$ & $09 \varepsilon^{\prime} \$ 80^{\prime} \mathrm{I} 69 \mathrm{~S}$ & $04 I^{2} 289^{2} z 21 S$ & $E E 9^{\prime} Z T^{\prime} \mathrm{T} D O S$ & $08 L^{2} T \angle T^{\prime} I S Z S$ & $20 t-x V$ \\
\hline os & $056^{\circ} 9 L \varepsilon^{\prime} Z L I^{2} I S$ & os & OS6'9LE'ZLI'TS & os & $00 Z^{2} 095^{\circ} \angle Z 8 S$ & $20 \mathrm{t}-\mathrm{ZV}$ \\
\hline $0 \$$ & $08 E^{\prime} 69 t^{\prime} \mathrm{C} 8 \mathrm{tS}$ & os & $08 \varepsilon^{2} 69 p^{6} \varepsilon 8+5$ & $0 s$ & $025^{\prime} 96 L^{\prime} 669 \$$ & I0I-ZV \\
\hline $4 \%$ & 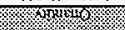 & F & Vim & 150 & 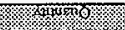 & \\
\hline 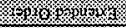 & 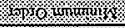 & 150 & 190 & \% & 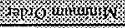 & \\
\hline \% & 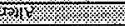 & 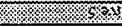 & 3218 & $\%$ & Hot & \\
\hline
\end{tabular}

\begin{tabular}{|c|c|c|c|c|c|c|}
\hline $20 \mathrm{~T}^{2} 20 \mathrm{~S}^{\circ} 0 L L S$ & $005^{2} 8 \tau S^{2} 8 L L^{\prime} I S$ & $088^{\prime} 85 \varepsilon^{\prime} 265 S$ & $005^{\prime} 82 S^{1} 8 L L^{\prime} 1 S^{\prime}$ & DII'S9Z'SISS & $005^{\circ} 825^{\circ} 8 L L^{*} I S$ & [010I \\
\hline $20 T^{\circ} 20 S^{\circ} 0 L \angle S$ & OLI'Z89'ZZIS & $088^{\prime} 8$ SE'Z6SS $^{\prime}$ & OLI'Z89'ZZIS & DII'S9I'SISS & $08 L^{\prime} T \angle I^{*} T S Z S$ & $20 I-\lambda Y$ \\
\hline os & OS6'9LE'ZLI'TS & $0 s$ & $0 \$ 6^{\prime} 9 L \varepsilon^{\prime} Z L I^{\prime} T \$$ & $0 S$ & 00Z'095'LZ8S & $\mathrm{ZOI} \cdot \mathrm{ZV}$ \\
\hline $0 s$ & $08 \varepsilon^{\prime} 69 t^{\prime} \mathrm{C} 8 \mathrm{tS}$ & 05 & $08 \varepsilon^{\top} 69 t^{t} \varepsilon 8 t^{\prime}$ & os & $025^{9} 96 L^{\prime} 6695$ & $10 \mathrm{I}^{-2 \mathrm{~V}}$ \\
\hline 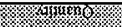 & Thys & Mnytas & xy & IEn & 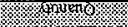 & \\
\hline (1) & (40) & 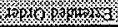 & $90 \%$ ormun & 19x & 1. & \\
\hline$\%$ & Ho & \% & Mitis & 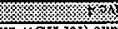 & Hrin & \\
\hline
\end{tabular}

\author{
(9 jo \ \\ • ( ${ }_{\text {s }}$ səo! Id-1!u

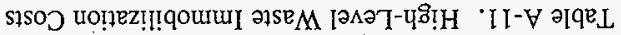 \\ 0 uols!n \\ $\varepsilon 00^{-V O V-צ M L-G S-J N H}$
}


HNF-SD-TWR-AGA-003

\section{Revision 0}

Table A-11. High-Level Waste Immobilization Costs

(Based on LMAES "Target-Unit-Prices").

(Sheet 6 of 6 )

\begin{tabular}{|c|c|c|c|c|}
\hline & \multicolumn{4}{|c|}{ Immobilization Cost Ratio (for HLW and LAW Services) $=$} \\
\hline & \multicolumn{2}{|c|}{ W } & \multicolumn{2}{|c|}{ 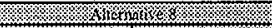 } \\
\hline & 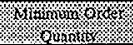 & 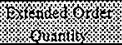 & Whom & Why \\
\hline $\mathrm{AZ}-101$ & $\$ 699,796,520$ & \$0 & $\$ 699,796,520$ & $\$ 0$ \\
\hline$A Z-102$ & $\$ 827,560,200$ & so & $\$ 1,078,731,980$ & $\$ 56,186,982$ \\
\hline AY-102 & $\$ 251,171,780$ & $\$ 693,408,336$ & so & $\$ 722,155,164$ \\
\hline Totn! & $\$ 1.778 .528 .500$ & $\$ 693,408,336$ & $\$ 1,778,528,500$ & $\$ 778,342,146$ \\
\hline
\end{tabular}

\begin{tabular}{|c|c|c|c|c|}
\hline & \multicolumn{4}{|c|}{ Immobilization Cost Ratio (for HLW and LAW Services) $=$} \\
\hline & \multicolumn{2}{|c|}{ 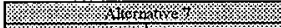 } & \multicolumn{2}{|c|}{ 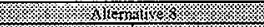 } \\
\hline & WVIM & KX) & M & Srorkd Xifor \\
\hline AZ-101 & $\$ 699,796,520$ & so & $\$ 699,796,520$ & $\$ 0$ \\
\hline$A Z-102$ & $\$ 827,560,200$ & so. & $\$ 1,078,731,980$ & $\$ 65,551,479$ \\
\hline AY-102 & $\$ 251,171,780$ & $\$ 808,976,392$ & $\$ 0$ & $\$ 842,514,358$ \\
\hline Total & $\$ 1,778,528,500$ & $\$ 808,976,392$ & $\$ 1,778,528,500$ & $\$ 908,065.837$ \\
\hline
\end{tabular}

\begin{tabular}{|c|c|c|c|c|}
\hline & \multicolumn{4}{|c|}{ Immobilization Cost Ratio (for HLW and LAW Services) $=$} \\
\hline & \multicolumn{2}{|c|}{ K K } & \multicolumn{2}{|c|}{ 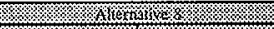 } \\
\hline & Kank & 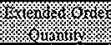 & 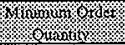 & 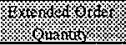 \\
\hline AZ-101 & $\$ 699,796,520$ & $\$ 0$ & $\$ 699,796,520$ & $\$ 0$ \\
\hline $\mathrm{AZ}-102$ & $\$ 327,560,200$ & $\$ 0$ & $\$ 1,078,731,980$ & $\$ 74,915,976$ \\
\hline AY-102 & $\$ 251,171,780$ & $\$ 924,544,448$ & $\$ 0$ & $\$ 962,873,552$ \\
\hline Total & $\$ 1,778,528,500$ & $\$ 924,544,448$ & $\$ 1.778 .528 .500$ & $\$ 1.037 .789 .528$ \\
\hline
\end{tabular}

\begin{tabular}{|c|c|c|c|c|}
\hline & \multicolumn{4}{|c|}{ Inunobilization Cost Ratio (for HLW and LAW Services) = } \\
\hline & \multicolumn{2}{|c|}{ W. } & \multicolumn{2}{|c|}{18 \% } \\
\hline & 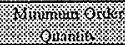 & xy & Whing & 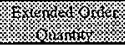 \\
\hline AZ-101 & $\$ 699,796,520$ & $\$ 0$ & $\$ 699,796,520$ & $\$ 0$ \\
\hline AZ-102 & $\$ 827,560,200$ & $\$ 0$ & $\$ 1,078,731,980$ & $\$ 84,280,473$ \\
\hline$A Y-102$ & $\$ 251,171,780$ & $\$ 1,040,112, \$ 04$ & $\$ 0$ & $\$ 1,083,232,746$ \\
\hline Total & $\$ 1.778,528,500$ & $\$ 1,040,112,504$ & $\$ 1.778 .528 .500$ & $\$ 1.167 .513 .219$ \\
\hline
\end{tabular}

\begin{tabular}{|c|c|c|c|c|}
\hline & \multirow{2}{*}{\multicolumn{2}{|c|}{ 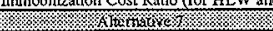 }} & \multirow{2}{*}{\multicolumn{2}{|c|}{ \% }} \\
\hline & & & & \\
\hline & Winnow & 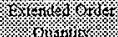 & Wun & Hon \\
\hline$A Z-101$ & $\$ 699,796,520$ & $\$ 0$ & $\$ 699,796,520$ & 50 \\
\hline$A 2-102$ & $\$ 827,560,200$ & so & $\$ 1,078,731,980$ & $\$ 93,644,970$ \\
\hline AY -102 & $\$ 251,171,780$ & $\$ 1,155,680,560$ & so & $\$ 1,203,591,940$ \\
\hline Total & $\$ 1,778,528,500$ & $\$ 1,155,680,560$ & $\$ 1.778,528,500$ & $\$ 1,297,236,910$ \\
\hline
\end{tabular}


HNF-SD-TWR-AGA-003

Revision 0
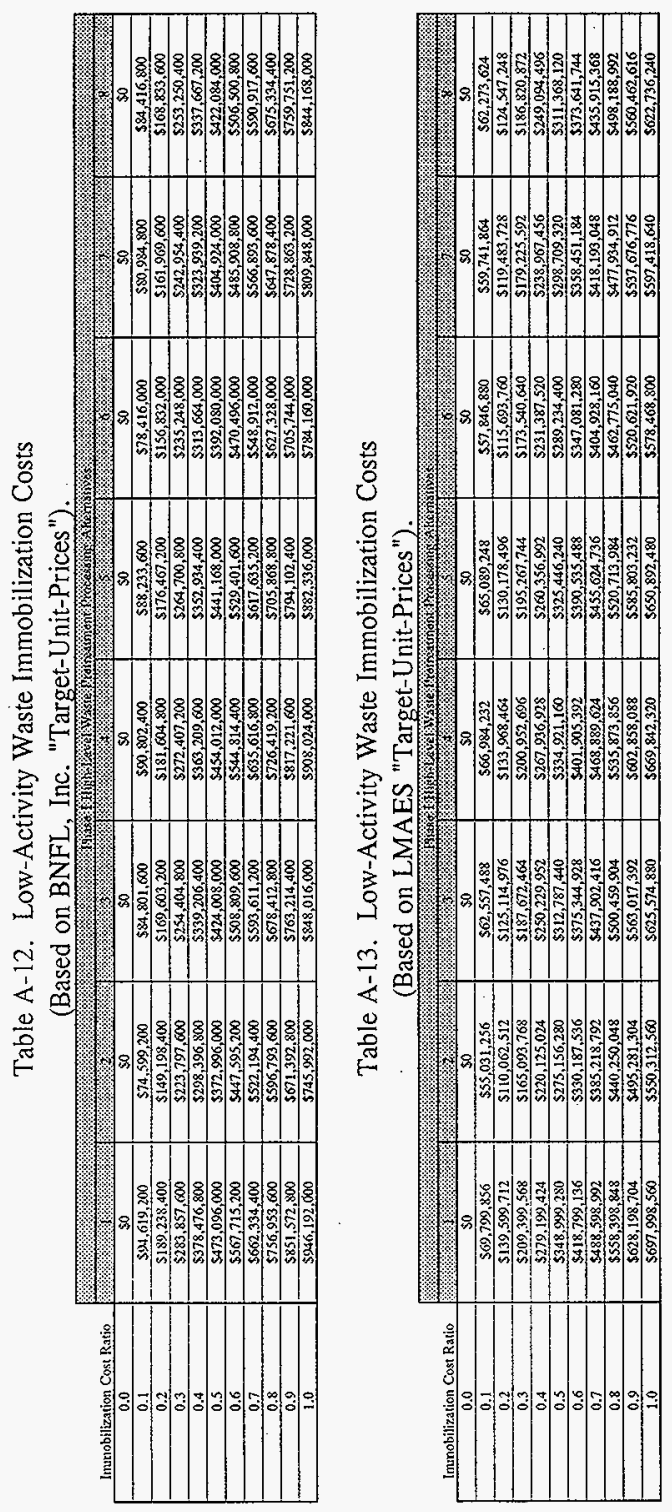
HNF-SD-TWR-AGA-003

Revision 0
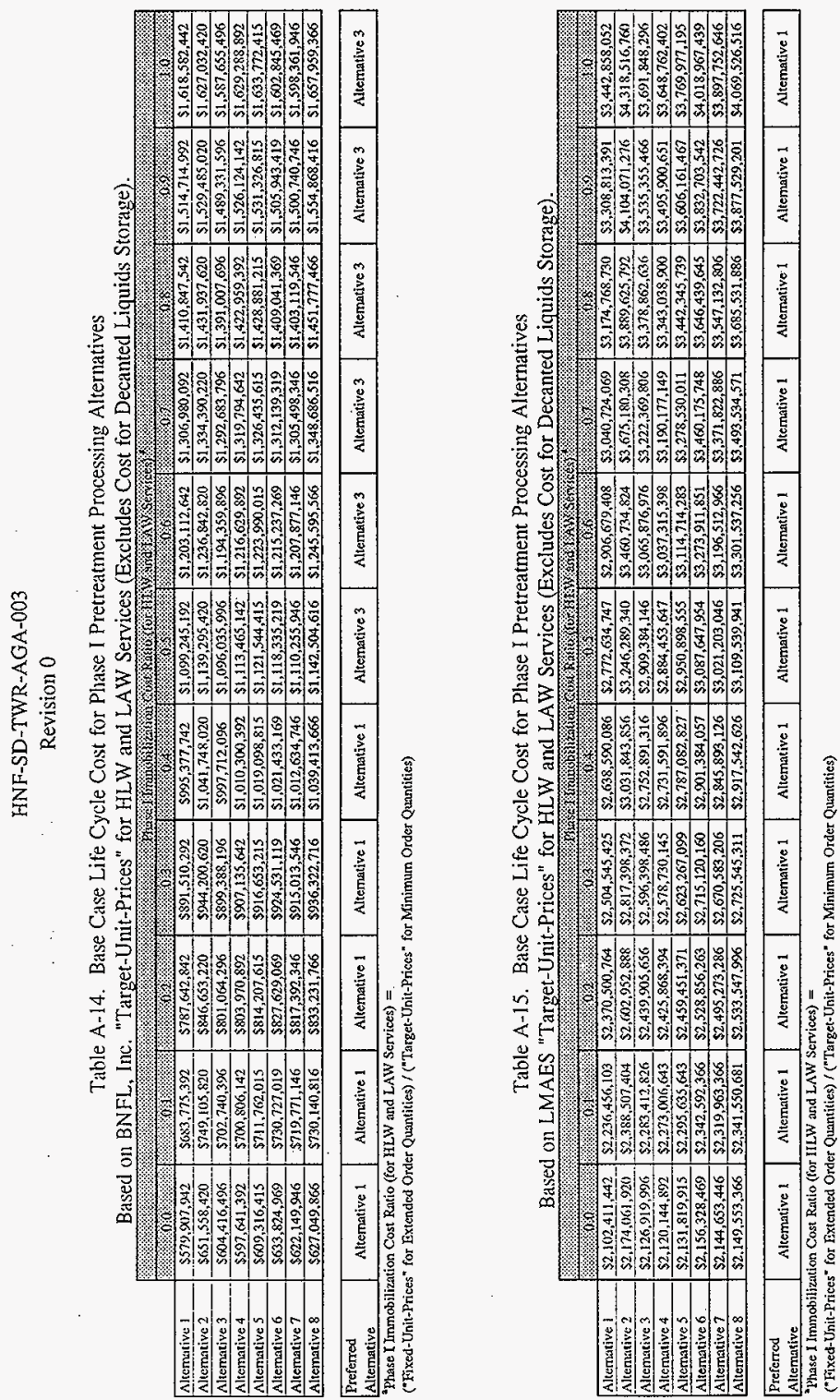
HNF-SD-TWR-AGA-003

Revision 0

Figures A-2 and A-3 are plots of the life-cycle costs by pretreatment processing alternative, based on assumed ratios of the "Fixed-Unit-Prices" for processing the extended order quantities of HLW and LAW feed to the "Target-Unit-Prices" for processing the minimum order quantities of HLW and LAW feed. 
Figure A-2. Base Case Life-Cycle Costs for High-Level Waste Pretreatment Processing Alternatives Based on BNFL, Inc. "Target-Unit-Prices" (Excludes Cost for Decanted Liquids Storage).

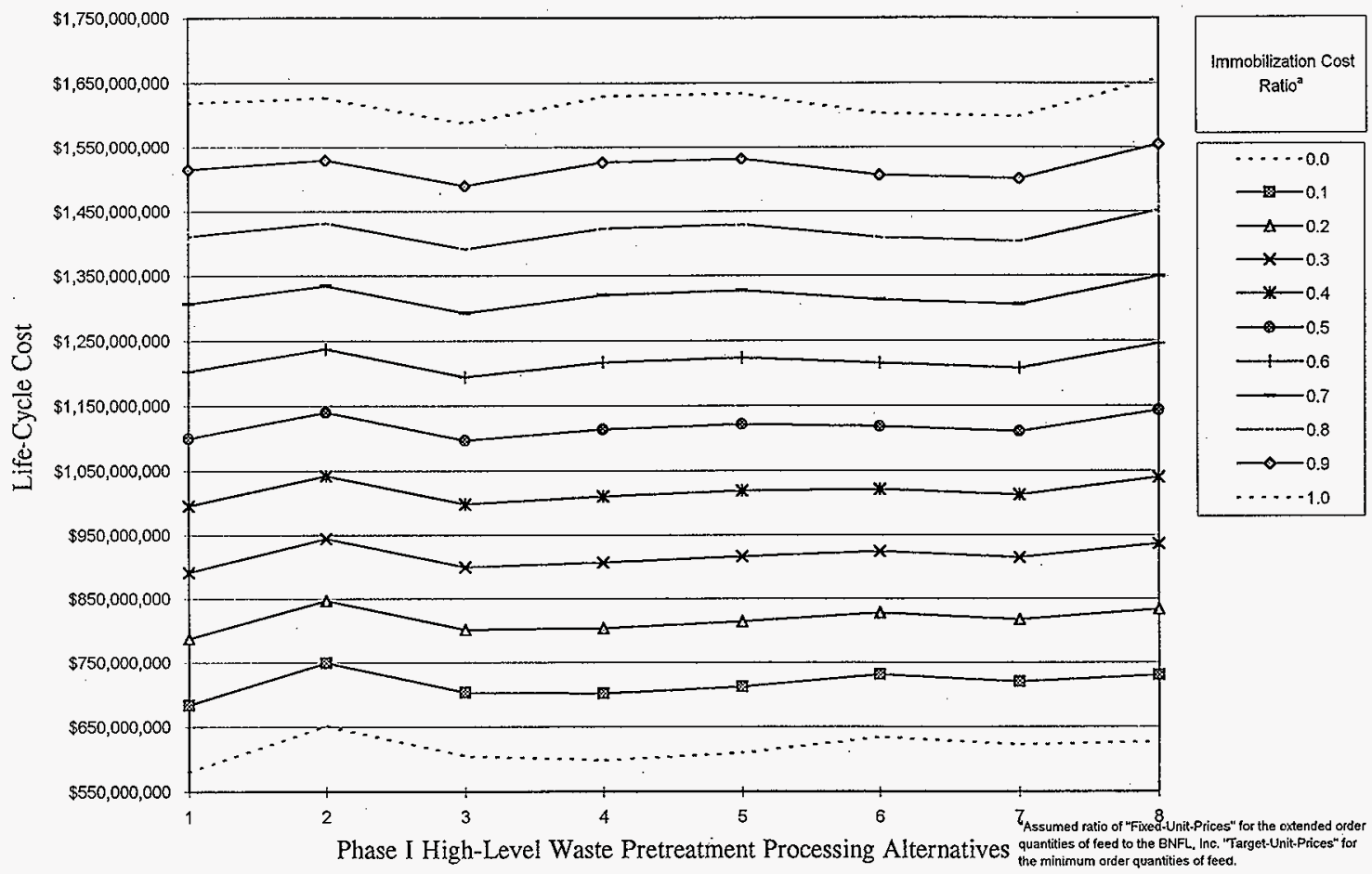


Figure A-3. Base Case Life-Cycle Costs for High-Level Waste Pretreatment Processing Alternatives Based on LMAES "Target-Unit-Prices" (Excludes Cost for Decanted Liquids Storage).

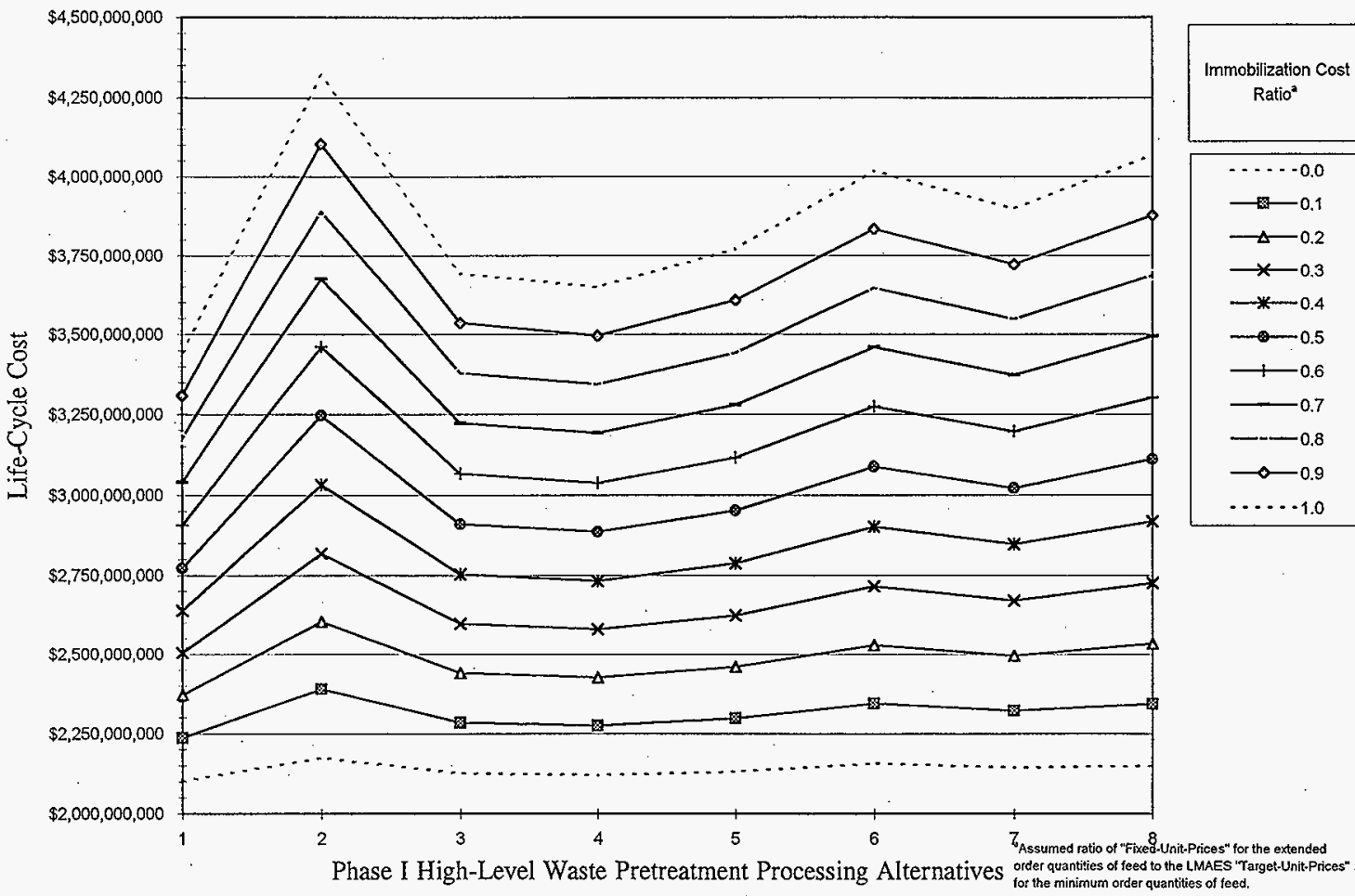


HNF-SD-TWR-AGA-003

Revision 0

This page intentionally left blank. 
HNF-SD-TWR-AGA-003

Revision 0

\section{APPENDIX B}

\section{SENSITIVITY STUDY RESULTS}


HNF-SD-TWR-AGA-003

Revision 0

This page intentionally left blank. 
HNF-SD-TWR-AGA-003

Revision 0

APPENDIX B

\section{SENSITIVITY STUDY RESULTS}

Two sensitivity studies are run for each Private Contractor. These analyze the effect of the immobilized high-level waste (HLW) product volume estimates on the life-cycle cost comparisons. The same approach for calculating life-cycle costs is used, and only the HLW product volumes are changed.

\section{B1.0 SUMMARY}

The base case life-cycle cost evaluation assumes that a decrease in the quantity of HLW feed results in a proportional decrease in the HLW product volume. (This effect is the result of assuming $25 \mathrm{wt} \%$ non-volatile oxides in the HLW product for all pretreatment scenarios). However, the actual HLW product loading achieved for the different pretreatment alternatives will depend on the HLW product formulation, or "what is limiting" the waste oxide loading. This appendix presents the HLW product volume estimates based on the basis of the following:

1. The minimum waste oxide loading (Base Case - plotted in Figures 3-1 and 3-2)

2. An assumption that there will be no reduction in the HLW product volume (Sensitivity Case \#1)

3. An assumption that the HLW product volume will be the same as those calculated using the glass property model estimates (Lambert et al. 1996) (Sensitivity Case \#2).

\section{B1.1 HIGH-LEVEL WASTE PRODUCT VOLUME ESTIMATES}

Table B- 1 shows the HLW product volumes used in the base case evaluation. 
HNF-SD-TWR-AGA-003

Revision 0

Table B-1. Estimated High-Level Waste Product Volumes for the Base Case

(Based on 25 wt\% Waste Oxides Excluding Sodium and Silicon).

\begin{tabular}{|l|c|c|c|c|c|c|c|c|}
\cline { 2 - 10 } \multicolumn{1}{c|}{} & \multicolumn{8}{c|}{ Alternatives } \\
\cline { 2 - 10 } \multicolumn{1}{c|}{} & 1 & 2 & 3 & 4 & 5 & 6 & 7 & 8 \\
\hline Product Volume $\left(\mathrm{m}^{3}\right)$ & 501 & 679 & 563 & 546 & 573 & 634 & 608 & 617 \\
\hline Canisters $\left(1.08-\mathrm{m}^{3}\right.$ capacity) & 464 & 629 & 521 & 506 & 531 & 587 & 563 & 571 \\
\hline
\end{tabular}

Table B-2 presents the HLW product volumes used for Sensitivity Case \#1. These values represent the bounding (or worst) case scenario, in which there is no reduction in the HLW product volume by enhanced sludge washing versus sludge washing.

Table B-2. Estimated High-Level Waste Product Volumes for Sensitivity Case \#1.

\begin{tabular}{|l|c|c|c|c|c|c|c|c|}
\cline { 2 - 7 } \multicolumn{1}{c|}{} & \multicolumn{8}{c|}{ Alternatives } \\
\cline { 2 - 6 } \multicolumn{1}{c|}{} & 1 & 2 & 3 & 4 & 5 & 6 & 7 & 8 \\
\hline Product Volume $\left(\mathrm{m}^{3}\right)$ & \multicolumn{8}{|c|}{629} \\
\hline Canisters $\left(1.08-\mathrm{m}^{3}\right.$ capacity) & \multicolumn{8}{c|}{} \\
\hline
\end{tabular}

Table B-3 presents the HLW product volumes based on the glass property models, used for Sensitivity Case \#2.

Table B-3. Estimated High-Level Waste Product Volumes for Sensitivity Case \#2 (Based on Glass Property Models from Lambert et al. [1996]).

\begin{tabular}{|l|c|c|c|c|c|c|c|c|}
\cline { 2 - 10 } \multicolumn{1}{c|}{} & \multicolumn{7}{c|}{ Alternatives } \\
\cline { 2 - 10 } \multicolumn{1}{c|}{} & 1 & 2 & 3 & 4 & 5 & 6 & 7 & 8 \\
\hline Product Volume $\left(\mathrm{m}^{3}\right)$ & 354 & 498 & 409 & 382 & 414 & 469 & 438 & 442 \\
\hline Canisters $\left(1.08-\mathrm{m}^{3}\right.$ capacity) & 328 & 461 & 379 & 354 & 383 & 434 & 406 & 409 \\
\hline
\end{tabular}




\section{Revision 0}

The values in Table B-3 correspond to the estimated maximum waste oxide loadings shown in Table B-4.

Table B-4. Estimated Maximum High-Level Waste Product Loadings ${ }^{\mathrm{a}, \mathrm{b}}$.

\begin{tabular}{|l|c|c|c|c|c|c|c|c|}
\cline { 2 - 9 } \multicolumn{1}{c|}{} & \multicolumn{8}{c|}{ Alternative } \\
\cline { 2 - 9 } \multicolumn{1}{c|}{} & 1 & 2 & 3 & 4 & 5 & 6 & 7 & 8 \\
\hline $241-\mathrm{AZ}-101$ & $33 \%$ & $35 \%$ & $33 \%$ & $35 \%$ & $33 \%$ & $33 \%$ & $35 \%$ & $35 \%$ \\
\hline $241-\mathrm{AZ}-102$ & $37 \%$ & $35 \%$ & $37 \%$ & $37 \%$ & $35 \%$ & $35 \%$ & $37 \%$ & $35 \%$ \\
\hline $\begin{array}{l}241-\mathrm{AY}-102 / \\
241-\mathrm{C}-106\end{array}$ & $35 \%$ & $34 \%$ & $34 \%$ & $35 \%$ & $35 \%$ & $34 \%$ & $34 \%$ & $35 \%$ \\
\hline
\end{tabular}

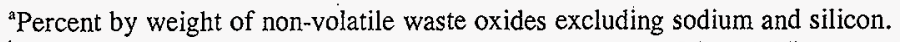

${ }^{b}$ As calculated by glass property models (Lambert et al. 1996) and (Hrma et al. 1994).

Figures B-1 through B-4 present the life cycle cost comparisons based on changing the HLW product volume estimates. 
Figure B-1. Life-Cycle Cost Comparison - Sensitivity Case \#1 (Based on BNFL, Inc. "Target-Unit-Prices").

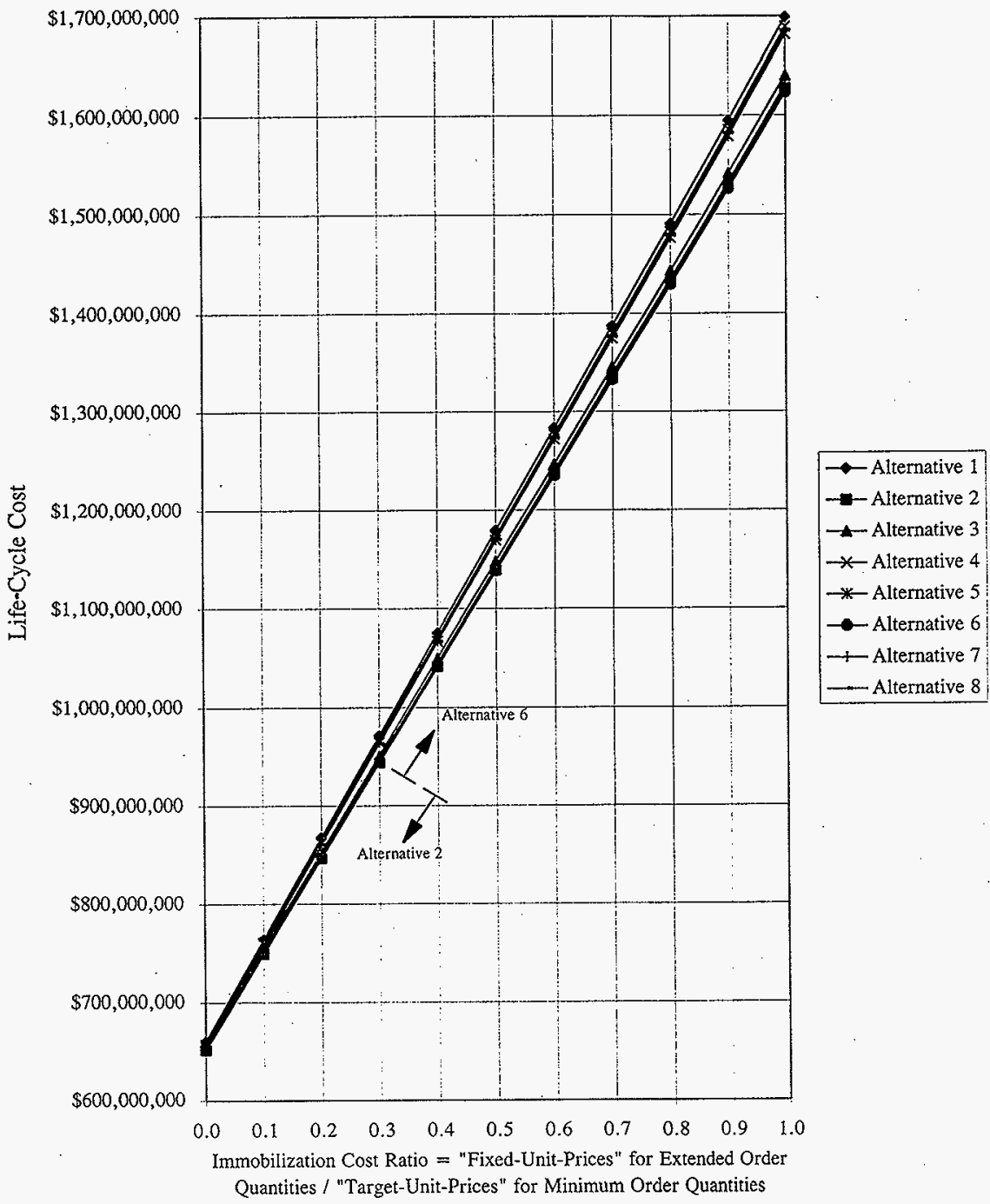




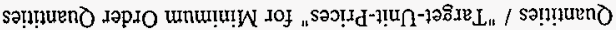

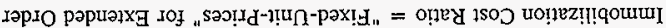

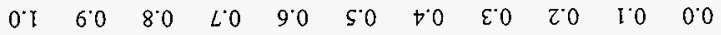

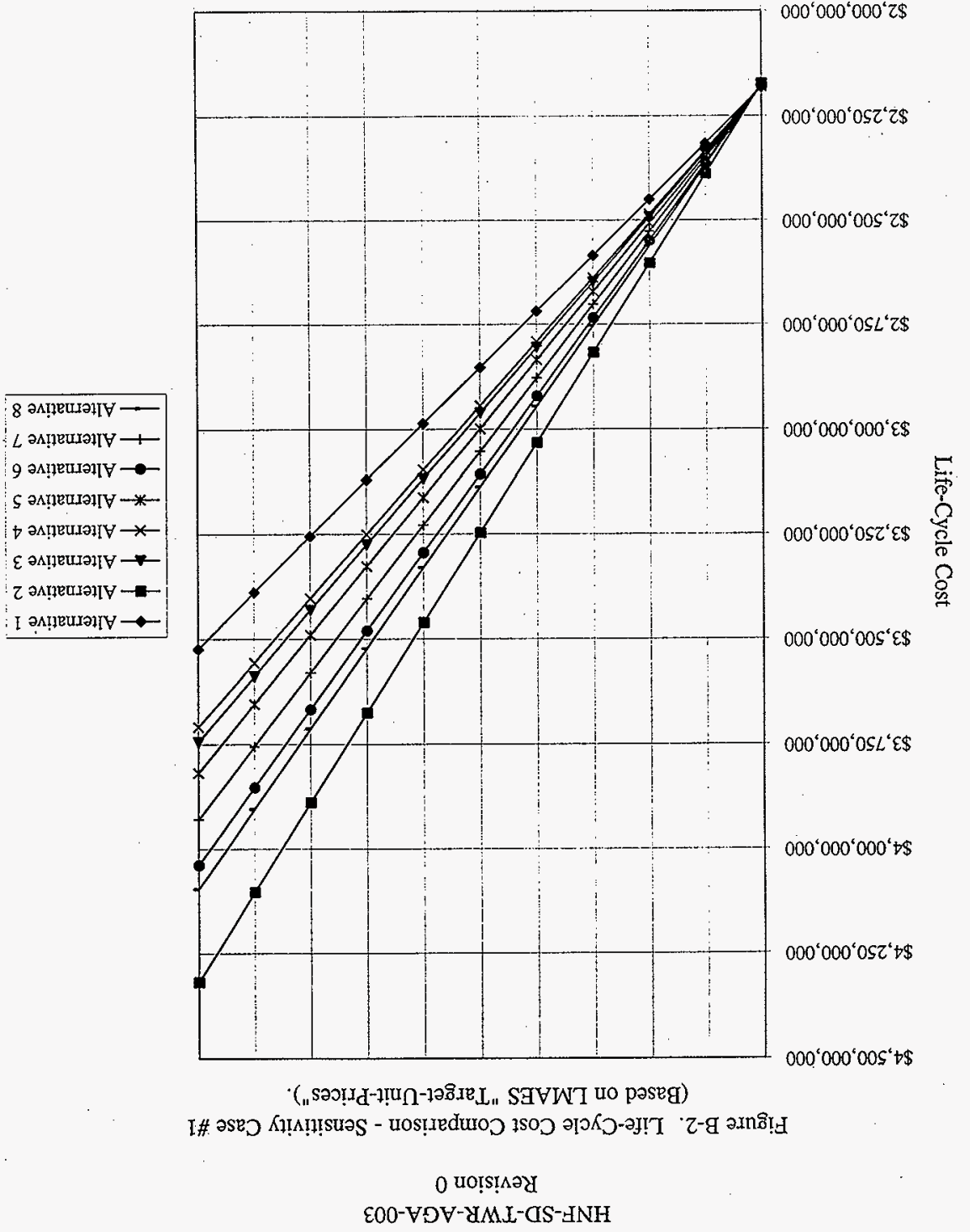




\section{HNF-SD-TWR-AGA-003 \\ Revision 0}

Figure B-3. Life-Cycle Cost Comparison - Sensitivity Case \#2 (Based on BNFL, Inc. "Target-Unit-Prices").

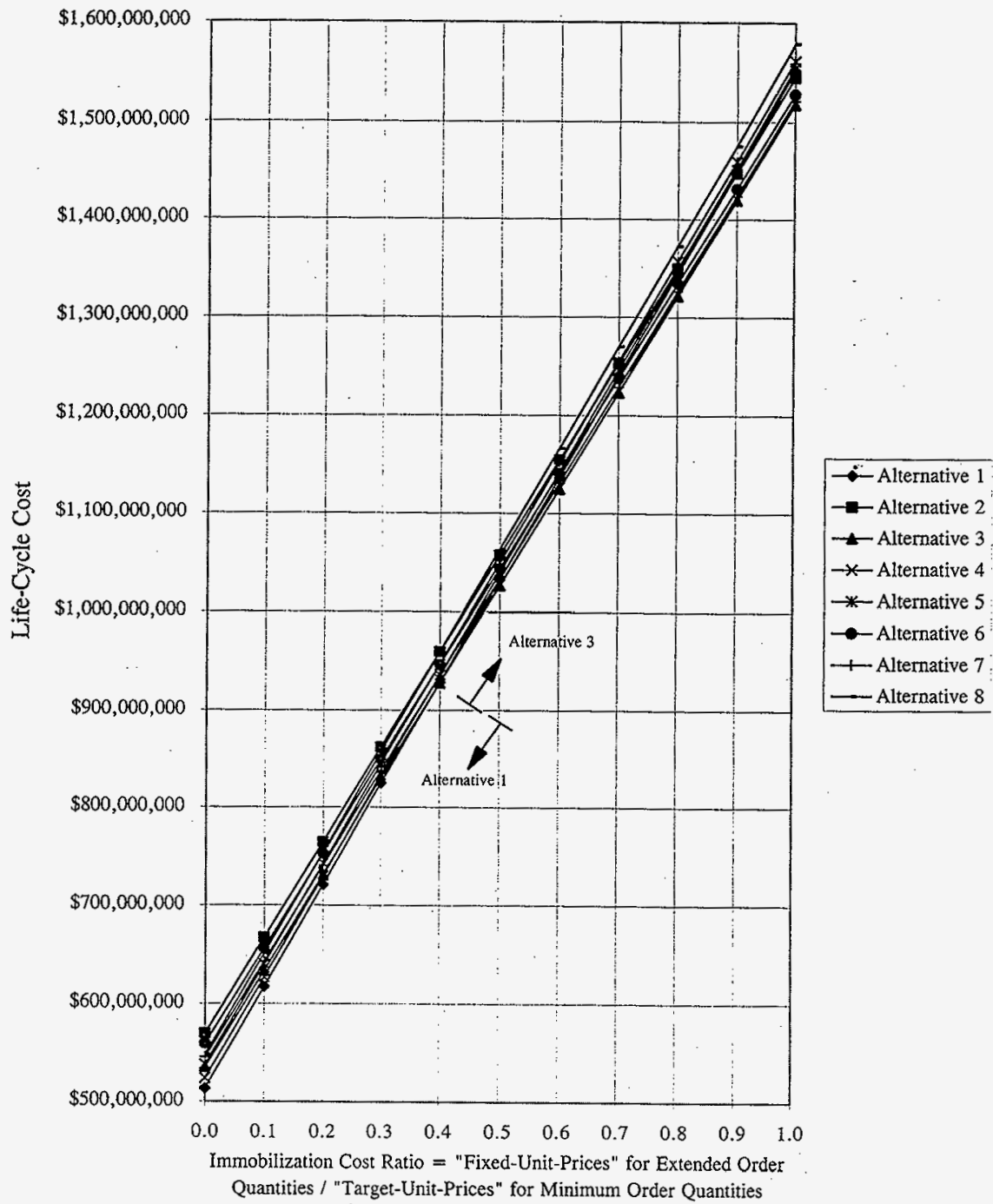




\section{Revision 0}

Figure B-4. Life-Cycle Cost Comparison - Sensitivity Case \#2 (Based on LMAES "Target-Unit-Prices").

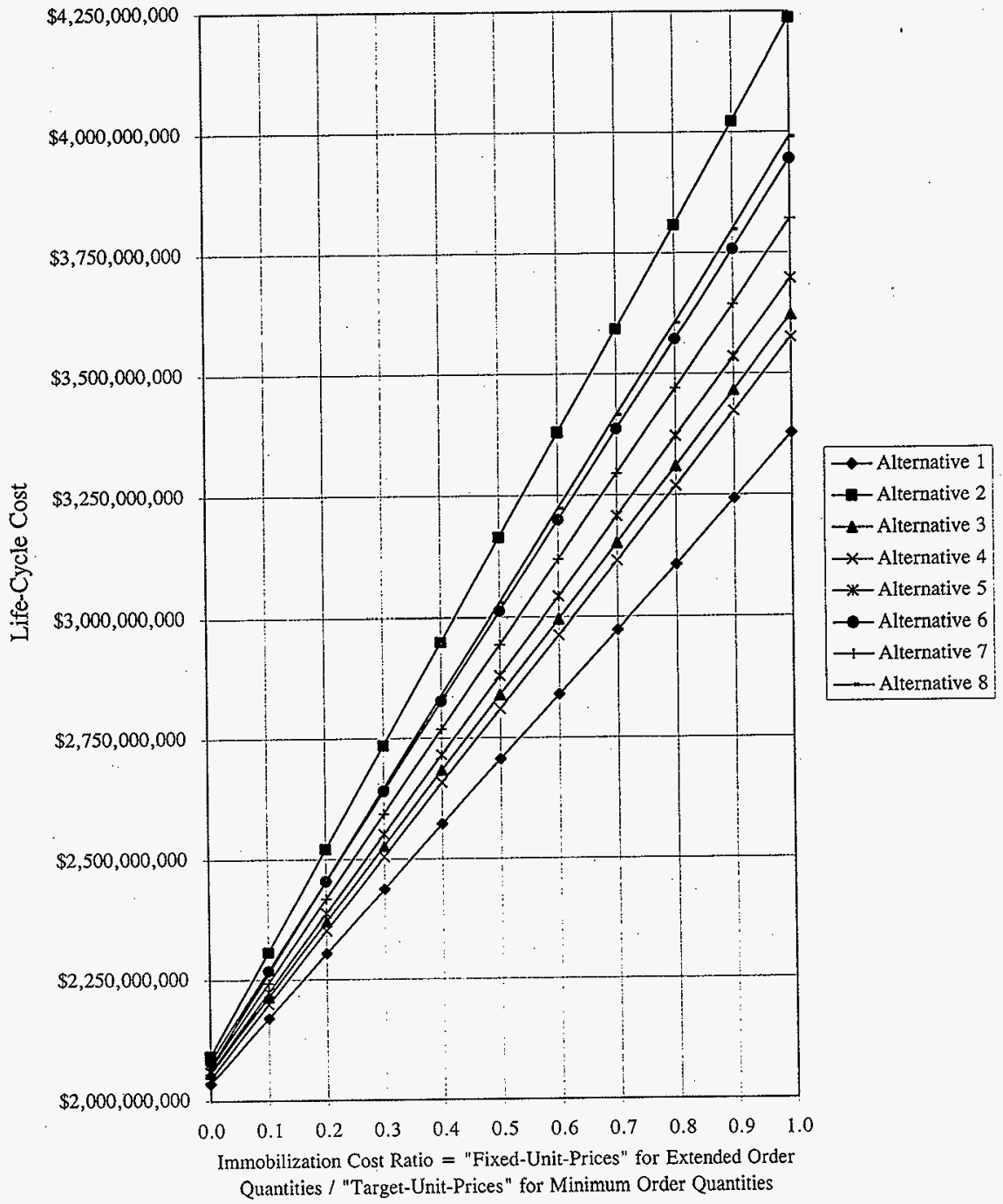


Figures B-1. and B-3 show that for BNFL, Inc., the alternatives still cannot be differentiated on the basis of life-cycle cost because of the small differences between life-cycle costs between the alternatives relative to the overall life-cycle cost. Table B-5 summarizes these comparisons.

Table B-5. Minimum Life-Cycle Cost Alternative(s) for BNFL, Inc. (Sensitivity Cases).

\begin{tabular}{|c|c|c|}
\hline Minimum cost alternative & $\begin{array}{c}\text { Immobilization Cost Ratio } \\
\text { (ICR) }\end{array}$ & $\begin{array}{c}\text { Assumed immobilized high- } \\
\text { level waste product volume } \\
\text { reduction (enhanced sludge } \\
\text { washing versus sludge } \\
\text { washing) }\end{array}$ \\
\hline 2 & $<0.3$ & None (Sensitivity Case \#1) \\
\hline 6 & $>0.3$ & $\begin{array}{c}\text { Equal to the difference in the } \\
\text { predicted HLW product } \\
\text { volumes using the glass } \\
\text { property models (Sensitivity } \\
\text { Case \#2) }\end{array}$ \\
\hline 1 & $<0.4$ & $>0.4$ \\
\hline
\end{tabular}

"All of the alternatives are within $\$ 50$ million of each other, which is negligible with respect to the overall life cycle cost.

"The "Immobilization Cost Ratio" (ICR), which ranges between 0.0 and 1.0, is defined as the ratio between the assumed "Fixed-Unit-Prices" for the extended order quantities of high-level waste and low-activity waste feed to the "Target-Unit-Prices" for the minimum order quantities of high-level waste and low-activity waste feed.

'Assumes the immobilized high-level waste product volumes are the same for both enhanced sludge washing and sludge washing.

Sensitivity Case \#2 gives a similar result as the Base Case (i.e., the minimum cost alternative is Alternative 1 or 3 ). This is because the glass property models show that the HLW oxide loading is roughly the same, regardless of the alternative (Table B-4).

As shown in Figures B-2 and B-4, for LMAES, the preferred alternative is always Alternative 1, regardless of the HLW disposal costs. The selection of the minimum cost alternative for the LMAES case is insensitive to the HLW disposal costs. 
HNF-SD-TWR-AGA-003

Revision 0

\section{APPENDIX C}

\section{MATERIAL BALANCE TABLES}

C-1 
HNF-SD-TWR-AGA-003

Revision 0

This page intentionally left blank. 
HNF-SD-TWR-AGA-003

Revision 0

APPENDIX C

MATERIAL BALANCE TABLES

\section{C1.0 HIGH-LEVEL WASTE FEED PREPARATION FLOWSHEETS}

The following pretreatment flowsheets have been developed for the preparation of the high-level waste (HLW) feeds for delivery to the Private Contractors. Schedules for each of these waste transfers are presented in Appendix E. This section summarizes the HLW feed preparation flowsheets for Alternatives 1 and 2.

The streams in Figure C-1 are numbered chronologically, beginning with Stream \#0, the sluicing retrieval of 241-C-106 into 241-AY-102. For simplicity, although some of the processing steps may actually overlap, as depicted in schedules provided in Appendix E, the steps are shown in the process flow diagram as occurring in series. The 3 DSTs are processed in the following sequence: $241-\mathrm{AZ}-101$-> 241-AZ-102 --> 241-AY-102/241-C-106. Table C-1 summarizes selected flowsheet results and operations that occur during sludge washing.

Table C-1. Phase I Material Balances.

\begin{tabular}{|c|c|c|c|c|c|c|}
\hline & $\begin{array}{c}\text { In-tank } \\
\text { evaporation }\end{array}$ & $\begin{array}{c}\text { Number of } \\
\text { leaches }\end{array}$ & $\begin{array}{c}\text { Number of } \\
\text { washes }\end{array}$ & $\begin{array}{c}\text { Number of } \\
\text { batches }\end{array}$ & $\begin{array}{c}\text { Batch volume } \\
(\mathrm{L})\end{array}$ & $\begin{array}{c}\text { Batch size } \\
\text { (MT) }\end{array}$ \\
\hline \multirow{2}{*}{241 -AZ-101 } & \multirow{2}{*}{ Yes } & 1 & 3 & 2 & 386,000 & 33.3 \\
\cline { 3 - 7 } & \multirow{2}{*}{ 241-AZ-102 } & 0 & 3 & 2 & 584,000 & 48.2 \\
\hline \multirow{2}{*}{ 241-AY-102 } & \multirow{2}{*}{ Yes } & 1 & 2 & 3 & 433,000 & 38.0 \\
\cline { 3 - 7 } & \multirow{2}{*}{ No } & 0 & 4 & 4 & 455,000 & 40.4 \\
\cline { 3 - 7 } & & 1 & 2 & 5 & 482,000 & 30.6 \\
\hline
\end{tabular}


HNF-SD-TWR-AGA-003

Revision 0

\section{C1.2 ALTERNATIVE 1}

\section{1-AZ-101 Sludge Pretreatment Process Summary}

It is assumed that the supernatant in 241-AZ-101 is in-tank concentrated to the $5 M$ sodium limit (Stream \#2a). It is also assumed that prior to any transfer of solids or supernatant, the solids have already been mobilized as part of the Project W-151 mixer pump testing. Mobilization of the solids results in a $20 \mathrm{wt} \%$ insoluble solids layer, after 30 days of settling.

A $50 \mathrm{wt} \%$ caustic solution (Stream \#4a) is added to $241-\mathrm{AZ}-101$ to reach a $1.0 \mathrm{M}$ hydroxide concentration in the liquid. The sludge is mixed and heated, followed by 30 days for solids settling. After the supernatant from AY-101 is decanted to AP-108 (Stream \#1, "Bootstrap Transfer"), the supernatant from 241-AZ-101 is decanted down to $0.25 \mathrm{~m}$ (10 inches) above the solids level, and routed into tank AY-101 (Stream \#6a) to provide Waste Envelope B feed. The AZ-101 sludge is subsequently washed three times (Streams \#4, \#5, and $\# 6$ are repeated 3 more times) with dilute caustic solution $(0.1 M \mathrm{NaOH}$ and $0.01 M$ $\mathrm{NaNO}_{2}$ ). The wash solutions are added in a.1:1 dilution ratio with the settled solids volume to minimize the volume and the impact on DST storage space.

The decanted liquids (Streams \#6b, c, and d) from 241-AZ-101 are sent to AW-105 to be later evaporated. Staging of liquids to the evaporator is not shown, but is modeled in the Hanford Tank Waste Operation Simulator (HTWOS) simulation.

In the final step for 241-AZ-101 (Stream \#7), enough transfer solution is added to the tank to dilute the solids to the maximum slurry concentration of $100 \mathrm{~g} / \mathrm{L}$ waste oxides. The slurry is transferred to the HLW immobilization facility in two batches (Stream \#8).

\section{1-AZ-102 Sludge Pretreatment Process Summary}

For 241-AZ-102, it is also assumed that the supernatant is in-tank concentrated to the $5 M$ sodium limit (Stream \#2b). A $50 \mathrm{wt} \%$ caustic solution (Stream \#10a) is added to the tank to reach a $1.0 \mathrm{M}$ hydroxide concentration in the liquid. After mixing and heating, followed by 30 days of solids settling, the supernatant is decanted to $0.25 \mathrm{~m}$ (10 in.) above the sludge. The decanted liquid is sent to 241-AY-101 (Waste Envelope B feed) and 241-AN-104. The tank is refilled with wash solution in a 3:1 dilution ratio with the settled solids volume.

The sludge is washed two times with dilute caustic solution in a $3: 1$ dilution ratio with the settled solids volume (Streams \#10,11, and 12 are repeated 2 more times). Decanted liquids (Streams \#12b and c) are sent to 241-AN-105. Transfer solution is added to achieve a $100 \mathrm{~g}$ of equivalent non-volatile oxides/L slurry, and the slurry is transferred to the HLW immobilization facility in three batches. 
HNF-SD-TWR-AGA-003

Revision 0

241-AY-102/241-C-106 Sludge Pretreatment Process Summary

Based on the current Project W-320 schedule, 100 percent of 241-C-106 is assumed to be present in 241-AY-102 prior to the start of pretreatment. After the retrieval of 241-C-106, a $50 \mathrm{wt} \%$ caustic solution (Stream \#16a) is added to the tank to reach a $1.0 \mathrm{M}$ hydroxide concentration in the liquid. After mixing and heating, followed by 30 days of solids settling, the supernatant is decanted down to $0.25 \mathrm{~m}$ (10 in.) above the settled solids layer. The sludge is then washed twice with a 1:1 ratio of wash solution to the settled solids volume.

The decanted liquids resulting from these pretreatment steps, including the initial decant, are routed to $241-\mathrm{AN}-105$. Transfer solution is added to dilute the waste to $100 \mathrm{~g}$ of equivalent non-volatile oxides per liter slurry. The transfer solution may have to be added inline due to a limited tank capacity. The slurry is delivered in five batches to the Private Contractor.

\section{C1.2 ALTERNATIVE 2}

\section{1-AZ-101 Sludge Pretreatment Process Summary}

It is assumed that the supernatant in 241-AZ-101 is in-tank concentrated to the $5 M$ sodium limit (Stream \#2a). It is also assumed that prior to any transfer of solids or supernatant, the solids have already been mobilized as part of the Project W-151 mixer pump testing. Mobilization of the solids results in a $20 \mathrm{wt} \%$ insoluble solids layer, after 30 days of settling.

After the supernatant from AY-101 is decanted to AP-108 (Stream \#1, "Bootstrap Transfer"), the supernatant from $241-\mathrm{AZ}-101$ is decanted down to $0.25 \mathrm{~m}$ (10 inches) above the solids level, and routed into tank 241-AY-101 (Stream \#3, "Bootstrap Transfer"). The 241-AZ-101 sludge is subsequently washed three times (Streams \#4, \#5, and \#6 are repeated 3 times) with dilute caustic solution $\left(0.1 \mathrm{M} \mathrm{NaOH}\right.$ and $\left.0.01 M \mathrm{NaNO}_{2}\right)$. The wash solutions are added in a 1:1 dilution ratio with the settled solids volume to minimize the volume and the impact on DST storage space.

The decanted liquids (Streams \#6a, b, and c) from 241-AZ-101 are sent to $241-\mathrm{AW}-105$ to be later evaporated. Staging of liquids to the evaporator is not shown, but is modelled in the HTWOS simulation.

In the final step for 241-AZ-101 (Stream \#7), enough transfer solution is added to the tank to dilute the solids to the maximum slurry concentration of $100 \mathrm{~g} / \mathrm{L}$ equivalent nonvolatile oxides. The slurry is transferred to the HLW immobilization facility in two batches (Stream \#8). 


\section{1-AZ-102 Sludge Pretreatment Process Summary}

For 241-AZ-102, it is also assumed that the supernatant is in-tank concentrated to the $5 M$ sodium limit (Stream \#2b). However, instead of the evaporation step being followed by sludge mobilization (mixer pump testing), the supernatant is decanted first to $0.25 \mathrm{~m}$ (10 in.) above the sludge, and the tank is refilled with wash solution in a 1:1 dilution ratio with the settled solids volume. The solution from the initial decant is split between tank 241-AY-101 and tank:241-AN-104.

The sludge is washed four times with dilute caustic solution in a 1:1 dilution ratio with the settled solids volume. Decanted liquids are sent to 241-AN-105. Transfer solution is added to achieve a $100 \mathrm{~g}$ of equivalent non-volatile oxides/L slurry, and the slurry is transferred to the HLW immobilization facility in four batches.

\section{1-AY-102/241-C-106 Sludge Pretreatment Process Summary}

Based on the current Project W-320 schedule, 100 percent of $241-\mathrm{C}-106$ is assumed to be present in 241-AY-102 prior to the start of pretreatment. After the retrieval of 241-C-106, the supernatant is decanted down to $0.25 \mathrm{~m}$ (10 in.) above the settled solids layer. The wash solution added in a 1:1 ratio with the settled solids volume and requires two washes.

The decanted liquids resulting from these pretreatment steps, including the initial decant, are routed to 241-AN-105. Transfer solution is added to dilute the waste to $100 \mathrm{~g}$ of waste oxides per liter slurry. The transfer solution may have to be added in-line due to a limited tank capacity. The slurry is delivered in five batches to the Private Contractor. 
HNF-SD-TWR-AGA-003

Revision 0

Table C-1. Alternative 1 Material Balance.

(Sheet 1 of 12)

\begin{tabular}{|l|c|c|c|c|c|c|c|c|}
\hline $\begin{array}{l}\text { STREAM NAME } \\
\text { LIQUID COMPONENTS }\end{array}$ & AZ101 & AZ102 & AY102 & 0 & 1 & $2 \mathrm{~A}$ & $4 \mathrm{~A}$ & $5 \mathrm{~A}$ \\
\hline Total Mass Flow (kg) & $3.88 \mathrm{E}+06$ & $3.79 \mathrm{E}+06$ & $3.71 \mathrm{E}+06$ & & & & $1.04 \mathrm{E}+05$ & $4.05 \mathrm{E}+06$ \\
\hline Volume (L) & $3.04 \mathrm{E}+06$ & $3.39 \mathrm{E}+06$ & $3.42 \mathrm{E}+06$ & & & & $6.76 \mathrm{E}+04$ & $3.09 \mathrm{E}+06$ \\
\hline Volume (gal) & $8.03 \mathrm{E}+05$ & $8.96 \mathrm{E}+05$ & $9.03 \mathrm{E}+05$ & & & & $1.79 \mathrm{E}+04$ & $8.18 \mathrm{E}+05$ \\
\hline Specific Gravity & $1.28 \mathrm{E}+00$ & $1.12 \mathrm{E}+00$ & $1.08 \mathrm{E}+00$ & & & & $1.54 \mathrm{E}+00$ & $1.31 \mathrm{E}+00$ \\
\hline
\end{tabular}

Radionuclides ( $\mathrm{Ci}$ )

\begin{tabular}{|l|l|l|l|l|l|l|l|l|}
\hline Cs-137 & $7.43 \mathrm{E}+06$ & $4.33 \mathrm{E}+06$ & $5.26 \mathrm{E}+05$ & & & & & $7.43 \mathrm{E}+06$ \\
\hline Ba-137 & $7.06 \mathrm{E}+06$ & $4.11 \mathrm{E}+06$ & $5.00 \mathrm{E}+05$ & & & & & $7.06 \mathrm{E}+06$ \\
\hline Sr-90 & $2.01 \mathrm{E}+04$ & $5.29 \mathrm{E}+03$ & & & & & & $2.01 \mathrm{E}+04$ \\
\hline Y-90 & $2.01 \mathrm{E}+04$ & $5.29 \mathrm{E}+03$ & & & & & & $2.01 \mathrm{E}+04$ \\
\hline Total Curies & $1.45 \mathrm{E}+07$ & $8.44 \mathrm{E}+06$ & $1.03 \mathrm{E}+06$ & & & & & $1.45 \mathrm{E}+07$ \\
\hline
\end{tabular}

Chemicals (kg)

\begin{tabular}{|l|c|c|c|c|c|c|c|c|}
\hline $\mathrm{Bi}+3$ & $0.00 \mathrm{E}+00$ & $1.75 \mathrm{E}+00$ & & & & & & \\
\hline $\mathrm{Ca}+2$ & $9.25 \mathrm{E}-01$ & $6.01 \mathrm{E}-01$ & $3.61 \mathrm{E}+00$ & & & & & $9.25 \mathrm{E}-01$ \\
\hline $\mathrm{Cs}+$ & $8.58 \mathrm{E}+01$ & $4.99 \mathrm{E}+01$ & $6.07 \mathrm{E}+00$ & & & & & $8.58 \mathrm{E}+01$ \\
\hline $\mathrm{Fe}+3$ & $3.91 \mathrm{E}-01$ & & - & & & & & $3.91 \mathrm{E}-01$ \\
\hline $\mathrm{K}+$ & $1.38 \mathrm{E}+04$ & $6.37 \mathrm{E}+03$ & $1.49 \mathrm{E}+03$ & & & & & $1.42 \mathrm{E}+04$ \\
\hline $\mathrm{Mg}+2$ & $0.00 \mathrm{E}+00$ & & $2.00 \mathrm{E}+00$ & & & & & \\
\hline $\mathrm{Mn}+4$ & $1.48 \mathrm{E}+01$ & $5.50 \mathrm{E}-01$ & $3.51 \mathrm{E}+01$ & & & & & $1.48 \mathrm{E}+01$ \\
\hline $\mathrm{Na}+$ & $3.59 \mathrm{E}+05$ & $1.97 \mathrm{E}+05$ & $1.65 \mathrm{E}+05$ & & & & $2.99 \mathrm{E}+04$ & $3.98 \mathrm{E}+05$ \\
\hline $\mathrm{Ni}+3$ & $4.11 \mathrm{E}-01$ & & & & & & & $4.11 \mathrm{E}-01$ \\
\hline $\mathrm{Si}+4$ & $1.97 \mathrm{E}+00$ & $5.28 \mathrm{E}+00$ & $3.35 \mathrm{E}+01$ & & & & & $1.97 \mathrm{E}+00$ \\
\hline $\mathrm{Sr}+2$ & $3.70 \mathrm{E}-01$ & $1.73 \mathrm{E}-01$ & & & & & & $3.70 \mathrm{E}-01$ \\
\hline $\mathrm{UO} 2+2$ & $0.00 \mathrm{E}+00$ & $9.42 \mathrm{E}+00$ & $4.04 \mathrm{E}+03$ & & & & & \\
\hline $\mathrm{Zr}+4$ & $9.33 \mathrm{E}+00$ & $1.64 \mathrm{E}+00$ & $1.82 \mathrm{E}-01$ & & & & & $9.33 \mathrm{E}+00$ \\
\hline $\mathrm{Al}(\mathrm{OH}) 4-$ & $1.18 \mathrm{E}+05$ & $9.25 \mathrm{E}+03$ & $4.62 \mathrm{E}+03$ & & & & & $1.70 \mathrm{E}+05$ \\
\hline $\mathrm{C} 2 \mathrm{O}-2$ & $5.89 \mathrm{E}+03$ & $2.20 \mathrm{E}+04$ & $4.13 \mathrm{E}+04$ & & & & & $2.24 \mathrm{E}+04$ \\
\hline $\mathrm{Cl}-$ & $6.40 \mathrm{E}+02$ & $3.17 \mathrm{E}+01$ & $1.18 \mathrm{E}+03$ & & & & & $6.40 \mathrm{E}+02$ \\
\hline $\mathrm{CO}-2$ & $6.26 \mathrm{E}+04$ & $8.05 \mathrm{E}+04$ & $1.20 \mathrm{E}+05$ & & & & & $6.26 \mathrm{E}+04$ \\
\hline $\mathrm{Cr}(\mathrm{OH}) 4-$ & $5.22 \mathrm{E}+01$ & $1.13 \mathrm{E}+01$ & $3.60 \mathrm{E}-01$ & & & & & $5.22 \mathrm{E}+01$ \\
\hline $\mathrm{CrO} 4-2$ & $5.12 \mathrm{E}+03$ & $5.50 \mathrm{E}+03$ & & & & & & $5.12 \mathrm{E}+03$ \\
\hline $\mathrm{F}-$ & $5.56 \mathrm{E}+03$ & $2.67 \mathrm{E}+03$ & $2.71 \mathrm{E}+02$ & & & & & $5.56 \mathrm{E}+03$ \\
\hline $\mathrm{HCO3}-$ & $0.00 \mathrm{E}+00$ & & $4.07 \mathrm{E}+04$ & & & & & \\
\hline $\mathrm{NO} 2-$ & $2.09 \mathrm{E}+05$ & $1.05 \mathrm{E}+05$ & $2.40 \mathrm{E}+04$ & & & & & $2.09 \mathrm{E}+05$ \\
\hline $\mathrm{NO} 3-$ & $2.42 \mathrm{E}+05$ & $7.98 \mathrm{E}+04$ & $2.96 \mathrm{E}+03$ & & & & & $2.42 \mathrm{E}+05$ \\
\hline $\mathrm{OH}-$ & $3.98 \mathrm{E}+04$ & $6.12 \mathrm{E}+03$ & $1.10 \mathrm{E}+04$ & & & & $2.21 \mathrm{E}+04$ & $5.26 \mathrm{E}+04$ \\
\hline $\mathrm{PO} 4-3$ & $4.12 \mathrm{E}+03$ & $6.04 \mathrm{E}+02$ & $6.66 \mathrm{E}+03$ & & & & & $4.12 \mathrm{E}+03$ \\
\hline $\mathrm{SO} 4-2$ & $5.77 \mathrm{E}+04$ & $6.21 \mathrm{E}+04$ & $6.77 \mathrm{E}+03$ & & & & & $5.77 \mathrm{E}+04$ \\
\hline $\mathrm{H} 2 \mathrm{O}$ & $2.76 \mathrm{E}+06$ & $3.21 \mathrm{E}+06$ & $3.28 \mathrm{E}+06$ & & & & $5.20 \mathrm{E}+04$ & $2.81 \mathrm{E}+06$ \\
\hline
\end{tabular}


HNF-SD-TWR-AGA-003

Revision 0

Table C-1. Alternative 1 Material Balance.

(Sheet 2 of 12)

\begin{tabular}{|l|c|c|c|c|c|c|c|c|}
\hline STREAM NAME & AZ101 & AZ102 & AY102 & 0 & 1 & $2 \mathrm{~A}$ & $4 \mathrm{~A}$ & $5 \mathrm{~A}$ \\
\hline SOLID COMPONENTS & & & & & & & & \\
\hline Total Mass Flow (kg) & $1.56 \mathrm{E}+05$ & $2.28 \mathrm{E}+05$ & $3.49 \mathrm{E}+05$ & & & & & $8.78 \mathrm{E}+04$ \\
\hline
\end{tabular}

Radionuclides (Ci)

\begin{tabular}{|l|l|l|l|l|l|l|l|l|}
\hline Pu-239 & $9.59 \mathrm{E}+02$ & $1.25 \mathrm{E}+03$ & $3.51 \mathrm{E}+03$ & & & & & $9.59 \mathrm{E}+02$ \\
\hline Sr-90 & $6.34 \mathrm{E}+06$ & $3.69 \mathrm{E}+06$ & $4.23 \mathrm{E}+06$ & & & & & $6.34 \mathrm{E}+06$ \\
\hline Y-90 & $6.34 \mathrm{E}+06$ & $3.69 \mathrm{E}+06$ & $4.23 \mathrm{E}+06$ & & & & & \\
\hline Total Curies & $1.27 \mathrm{E}+07$ & $7.38 \mathrm{E}+06$ & $8.46 \mathrm{E}+06$ & & & & & $1.27 \mathrm{E}+06$ \\
\hline
\end{tabular}

Chemicals (kg)

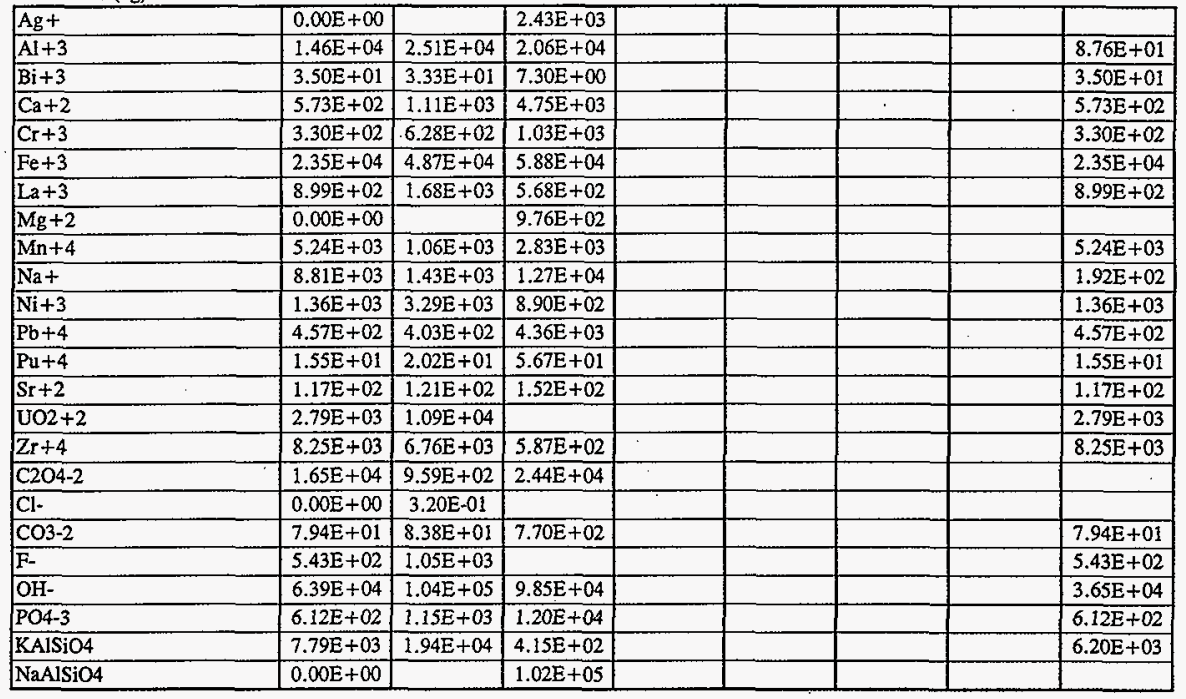


HNF-SD-TWR-AGA-003

Revision 0

Table C-1. Alternative 1 Material Balance.

(Sheet 3 of 12)

\begin{tabular}{|l|c|c|c|c|c|c|c|c|}
\hline $\begin{array}{l}\text { STREAM NAME } \\
\text { LIQUID COMPONENTS }\end{array}$ & $6 \mathrm{~A}$ & $4 \mathrm{~B}$ & $5 \mathrm{~B}$ & $6 \mathrm{~B}$ & $4 \mathrm{C}$ & $5 \mathrm{C}$ & $6 \mathrm{C}$ & $4 \mathrm{D}$ \\
\hline Total Mass Flow (kg) & $3.54 \mathrm{E}+06$ & $5.55 \mathrm{E}+05$ & $1.07 \mathrm{E}+06$ & $5.78 \mathrm{E}+05$ & $5.55 \mathrm{E}+05$ & $1.05 \mathrm{E}+06$ & $5.65 \mathrm{E}+05$ & $5.55 \mathrm{E}+05$ \\
\hline Volume (L) & $2.70 \mathrm{E}+06$ & $5.53 \mathrm{E}+05$ & $9.45 \mathrm{E}+05$ & $5.12 \mathrm{E}+05$ & $5.53 \mathrm{E}+05$ & $9.86 \mathrm{E}+05$ & $5.33 \mathrm{E}+05$ & $5.53 \mathrm{E}+05$ \\
\hline Volume (gal) & $7.14 \mathrm{E}+05$ & $1.46 \mathrm{E}+05$ & $2.50 \mathrm{E}+05$ & $1.35 \mathrm{E}+05$ & $1.46 \mathrm{E}+05$ & $2.61 \mathrm{E}+05$ & $1.41 \mathrm{E}+05$ & $1.46 \mathrm{E}+05$ \\
\hline Specific Gravity & $1.31 \mathrm{E}+00$ & $1.00 \mathrm{E}+00$ & $1.13 \mathrm{E}+00$ & $1.13 \mathrm{E}+00$ & $1.00 \mathrm{E}+00$ & $1.06 \mathrm{E}+00$ & $1.06 \mathrm{E}+00$ & $1.00 \mathrm{E}+00$ \\
\hline
\end{tabular}

Radionuclides (Ci)

\begin{tabular}{|l|l|l|l|l|l|l|l|l|}
\hline Cs-137 & $6.49 \mathrm{E}+06$ & & $9.42 \mathrm{E}+05$ & $5.10 \mathrm{E}+05$ & & $4.32 \mathrm{E}+05$ & $2.33 \mathrm{E}+05$ & \\
\hline $\mathrm{Ba}-137$ & $6.16 \mathrm{E}+06$ & & $8.94 \mathrm{E}+05$ & $4.84 \mathrm{E}+05$ & & $4.10 \mathrm{E}+05$ & $2.22 \mathrm{E}+05$ & \\
\hline Sr-90 & $1.76 \mathrm{E}+04$ & & $2.55 \mathrm{E}+03$ & $1.38 \mathrm{E}+03$ & & $1.17 \mathrm{E}+03$ & $6.32 \mathrm{E}+02$ & \\
\hline Y-90 & $1.76 \mathrm{E}+04$ & & $2.55 \mathrm{E}+03$ & $1.38 \mathrm{E}+03$ & & $1.17 \mathrm{E}+03$ & $6.32 \mathrm{E}+02$ & \\
\hline Total Curies & $1.27 \mathrm{E}+07$ & & $1.84 \mathrm{E}+06$ & $9.97 \mathrm{E}+05$ & & $8.44 \mathrm{E}+05$ & $4.57 \mathrm{E}+05$ & \\
\hline
\end{tabular}

Chemicals $(\mathrm{kg})$

\begin{tabular}{|c|c|c|c|c|c|c|c|c|}
\hline $\mathrm{Bi}+3$ & & & $0.00 \mathrm{E}+00$ & & & & & \\
\hline $\mathrm{Ca}+2$ & $8.07 \mathrm{E}-01$ & & $1.17 \mathrm{E}-01$ & $6.34 \mathrm{E}-02$ & & $5.37 \mathrm{E}-02$ & $2.91 \mathrm{E}-02$ & \\
\hline Cst & $7.49 \mathrm{E}+01$ & & $1.09 \mathrm{E}+01$ & $5.89 \mathrm{E}+00$ & & $4.99 \mathrm{E}+00$ & $2.70 \mathrm{E}+00$ & \\
\hline $\mathrm{Fe}+3$ & $3.41 \mathrm{E}-01$ & & $4.95 \mathrm{E}-02$ & $2.68 \mathrm{E}-02$ & & $2.27 \mathrm{E}-02$ & $1.23 \mathrm{E}-02$ & \\
\hline $\mathrm{K}+$ & $1.24 \mathrm{E}+04$ & & $1.80 \mathrm{E}+03$ & $9.75 \mathrm{E}+02$ & & $8.26 \mathrm{E}+02$ & $4.47 \mathrm{E}+02$ & \\
\hline $\mathrm{Mg}+2$ & & & $0.00 \mathrm{E}+00$ & & & & & \\
\hline $\mathrm{Mn}+4$ & $1.29 \mathrm{E}+01$ & & $1.87 \mathrm{E}+00$ & $1.01 E+00$ & & $8.59 \mathrm{E}-01$ & $4.64 \mathrm{E}-01$ & \\
\hline $\mathrm{Na}+$ & $3.48 \mathrm{E}+05$ & $1.40 \mathrm{E}+03$ & $5.18 \mathrm{E}+04$ & $2.81 E+04$ & $1.40 \mathrm{E}+03$ & $2.52 \mathrm{E}+04$ & $1.36 \mathrm{E}+04$ & $1.40 \mathrm{E}+03$ \\
\hline $\mathrm{Ni}+3$ & $3.59 \mathrm{E}-01$ & & $5.21 \mathrm{E}-02$ & $2.82 \mathrm{E}-02$ & & $2.39 \mathrm{E}-02$ & $1.29 \mathrm{E}-02$ & \\
\hline $\mathrm{Si}+4$ & $1.72 \mathrm{E}+00$ & & $2.50 \mathrm{E}-01$ & $1.35 \mathrm{E}-01$ & & $1.15 \mathrm{E}-01$ & $6.19 \mathrm{E}-02$ & \\
\hline $\mathrm{Sr}+2$ & $3.23 \mathrm{E}-01$ & & $4.69 \mathrm{E}-02$ & $2.54 \mathrm{E}-02$ & & $2.15 \mathrm{E}-02$ & $1.16 \mathrm{E}-02$ & \\
\hline $\mathrm{UO} 2+2$ & & & $0.00 \mathrm{E}+00$ & & & & & \\
\hline $\mathrm{Zr}+4$ & $8.15 \mathrm{E}+00$ & & $1.18 \mathrm{E}+00$ & $6.40 \mathrm{E}-01$ & & $5.42 \mathrm{E}-01$ & $2.93 \mathrm{E}-01$ & \\
\hline $\mathrm{Al}(\mathrm{OH}) 4$ & $1.48 \mathrm{E}+05$ & & $2.15 \mathrm{E}+04$ & $1.16 \mathrm{E}+04$ & & $9.85 E+03$ & $5.33 \mathrm{E}+03$ & \\
\hline C2O4-2 & $1.96 \mathrm{E}+04$ & & $2.84 \mathrm{E}+03$ & $1.54 \mathrm{E}+03$ & & $1.30 \mathrm{E}+03$ & $7.04 \mathrm{E}+02$ & \\
\hline $\mathrm{Cl}-$ & $5.59 \mathrm{E}+02$ & & $8.11 \mathrm{E}+01$ & $4.39 \mathrm{E}+01$ & & $3.72 \mathrm{E}+01$ & $2.01 \mathrm{E}+01$ & \\
\hline $\mathrm{CO} 3-2$ & $5.46 \mathrm{E}+04$ & & $7.93 \mathrm{E}+03$ & $4.29 E+03$ & & $3.64 \mathrm{E}+03$ & $1.97 \mathrm{E}+03$ & \\
\hline $\mathrm{Cr}(\mathrm{OH}) 4-$ & $4.56 \mathrm{E}+01$ & & $6.61 \mathrm{E}+00$ & $3.58 \mathrm{E}+00$ & & $3.03 \mathrm{E}+00$ & $1.64 \mathrm{E}+00$ & \\
\hline CrO4-2 & $4.47 \mathrm{E}+03$ & & $6.48 \mathrm{E}+02$ & $3.51 \mathrm{E}+02$ & & $2.97 \mathrm{E}+02$ & $1.61 \mathrm{E}+02$ & \\
\hline F. & $4.85 \mathrm{E}+03$ & & $7.04 \mathrm{E}+02$ & $3.81 E+02$ & & $3.23 \mathrm{E}+02$ & $1.75 \mathrm{E}+02$ & \\
\hline HCO3- & & & $0.00 \mathrm{E}+00$ & & & & & \\
\hline NO2- & $1.83 \mathrm{E}+05$ & $2.54 \mathrm{E}+02$ & $2.68 \mathrm{E}+04$ & $1.45 E+04$ & $2.54 \mathrm{E}+02$ & $1.25 \mathrm{E}+04$ & $6.77 \mathrm{E}+03$ & $2.54 \mathrm{E}+02$ \\
\hline NO3- & $2.11 E+05$ & & $3.07 \mathrm{E}+04$ & $1.66 \mathrm{E}+04$ & & $1.41 \mathrm{E}+04$ & $7.60 \mathrm{E}+03$ & \\
\hline $\mathrm{OH}-$ & $4.60 \mathrm{E}+04$ & $9.40 \mathrm{E}+02$ & $7.61 E+03$ & $4.12 \mathrm{E}+03$ & $9.40 \mathrm{E}+02$ & $4.43 \mathrm{E}+03$ & $2.40 \mathrm{E}+03$ & $9.40 \mathrm{E}+02$ \\
\hline PO4-3 & $3.60 \mathrm{E}+03$ & & $5.22 \mathrm{E}+02$ & $2.83 \mathrm{E}+02$ & & $2.40 \mathrm{E}+02$ & $1.30 \mathrm{E}+02$ & \\
\hline SO4-2 & $5.04 \mathrm{E}+04$ & & $7.31 \mathrm{E}+03$ & $3.96 \mathrm{E}+03$ & & $3.35 \mathrm{E}+03$ & $1.81 \mathrm{E}+03$ & \\
\hline $\mathrm{H} 2 \mathrm{O}$ & $2.45 \mathrm{E}+06$ & $5.52 \mathrm{E}+05$ & $9.08 \mathrm{E}+05$ & $4.92 \mathrm{E}+05$ & $5.52 \mathrm{E}+05$ & $9.69 \mathrm{E}+0 \mathrm{~S}$ & $5.24 \mathrm{E}+05$ & $5.52 \mathrm{E}+05$ \\
\hline
\end{tabular}


HNF-SD-TWR-AGA-003

Revision 0

Table C-1. Alternative 1 Material Balance.

(Sheet 4 of 12)

\begin{tabular}{|l|c|c|c|c|c|c|c|c|}
\hline STREAM NAME & $6 \mathrm{~A}$ & $4 \mathrm{~B}$ & $5 \mathrm{~B}$ & $6 \mathrm{~B}$ & $4 \mathrm{C}$ & $5 \mathrm{C}$ & $6 \mathrm{C}$ & $4 \mathrm{D}$ \\
\hline SOLID COMPONENTS & & & & & & & & \\
\hline Total Mass Flow $(\mathrm{kg})$ & $2.90 \mathrm{E}+02$ & & $8.75 \mathrm{E}+04$ & $2.89 \mathrm{E}+02$ & & $8.72 \mathrm{E}+04$ & $2.88 \mathrm{E}+02$ & \\
\hline
\end{tabular}

Radionuclides ( $\mathrm{Ci}$ )

\begin{tabular}{|c|c|c|c|c|c|}
\hline $\mathrm{Pu}-239$ & $3.16 \mathrm{E}+00$ & $9.56 E+02$ & $3.15 \mathrm{E}+00$ & $9.53 \mathrm{E}+02$ & $3.14 \mathrm{E}+00$ \\
\hline Sr-90 & $2.09 \mathrm{E}+04$ & $6.31 \mathrm{E}+06$ & $2.08 \mathrm{E}+04$ & $6.29 \mathrm{E}+06$ & $2.08 \mathrm{E}+04$ \\
\hline $\mathrm{X}-90$ & $2.09 \mathrm{E}+04$ & $6.31 \mathrm{E}+06$ & $2.08 \mathrm{E}+04$ & $6.29 \mathrm{E}+06$ & $2.08 \mathrm{E}+04$ \\
\hline Total Curies & $4.18 \mathrm{E}+04$ & $1.26 \mathrm{E}+07$ & $4.17 \mathrm{E}+04$ & $1.26 \mathrm{E}+07$ & $4.15 \mathrm{E}+04$ \\
\hline
\end{tabular}

Chemicals (kg)

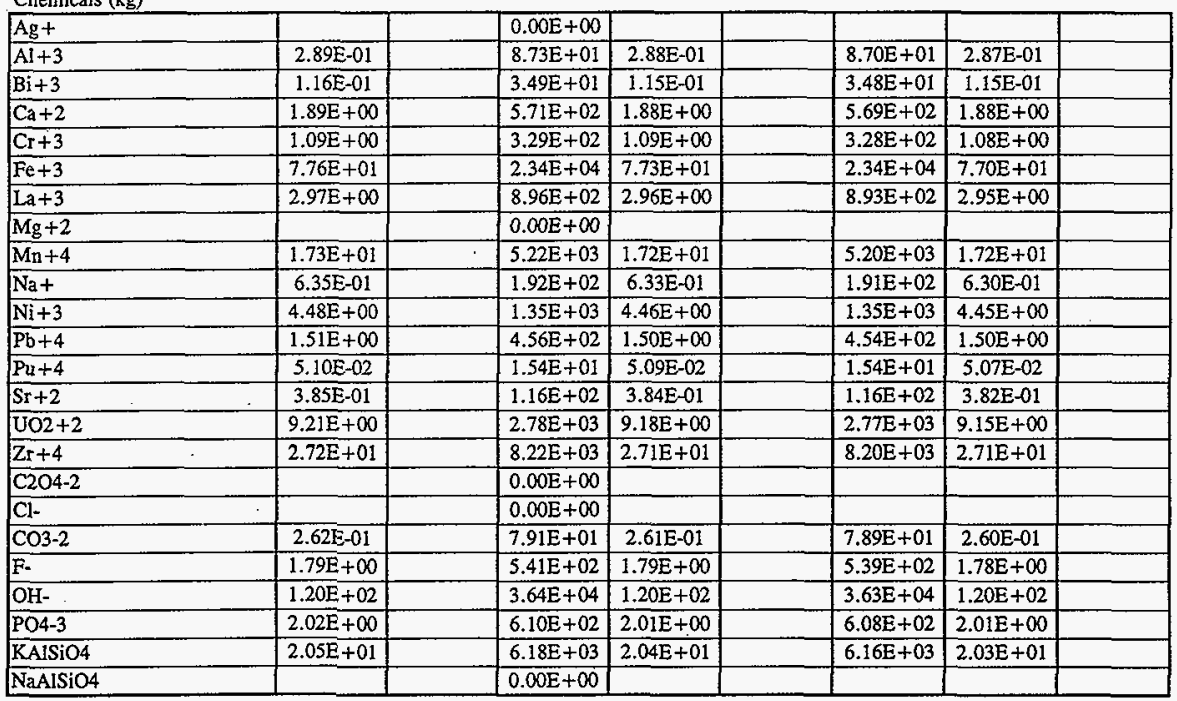


HNF-SD-TWR-AGA-003

Revision 0

Table C-1. Alternative 1 Material Balance.

(Sheet 5 of 12)

\begin{tabular}{|l|c|c|c|c|c|c|c|c|}
\hline $\begin{array}{l}\text { STREAM NAME } \\
\text { LIQUID COMPONENTS }\end{array}$ & $5 \mathrm{D}$ & $6 \mathrm{D}$ & 7 & 8 & $2 \mathrm{~B}$ & $10 \mathrm{~A}$ & $11 \mathrm{~A}$ & $12 \mathrm{~A}$ \\
\hline Total Mass Flow (kg) & $1.04 \mathrm{E}+06$ & $5.60 \mathrm{E}+05$ & $3.10 \mathrm{E}+05$ & $7.85 \mathrm{E}+05$ & $1.68 \mathrm{E}+06$ & $1.90 \mathrm{E}+05$ & $2.38 \mathrm{E}+06$ & $1.61 \mathrm{E}+06$ \\
\hline Volume (L) & $1.01 \mathrm{E}+06$ & $5.44 \mathrm{E}+05$ & $3.10 \mathrm{E}+05$ & $7.72 \mathrm{E}+05$ & $1.68 \mathrm{E}+06$ & $1.24 \mathrm{E}+05$ & $1.79 \mathrm{E}+06$ & $1.21 \mathrm{E}+06$ \\
\hline Volume (gal) & $2.66 \mathrm{E}+05$ & $1.44 \mathrm{E}+05$ & $8.19 \mathrm{E}+04$ & $2.04 \mathrm{E}+05$ & $4.44 \mathrm{E}+05$ & $3.27 \mathrm{E}+04$ & $4.74 \mathrm{E}+05$ & $3.20 \mathrm{E}+05$ \\
\hline Specific Gravity & $1.03 \mathrm{E}+00$ & $1.03 \mathrm{E}+00$ & $1.00 \mathrm{E}+00$ & $1.02 \mathrm{E}+00$ & $1.00 \mathrm{E}+00$ & $1.54 \mathrm{E}+00$ & $1.33 \mathrm{E}+00$ & $1.33 \mathrm{E}+00$ \\
\hline
\end{tabular}

Radionuclides (Ci)

\begin{tabular}{|l|l|l|l|l|l|l|l|l|}
\hline Cs-137 & $1.98 \mathrm{E}+05$ & $1.07 \mathrm{E}+05$ & & $9.10 \mathrm{E}+04$ & $0.00 \mathrm{E}+00$ & & $4.33 \mathrm{E}+06$ & $2.92 \mathrm{E}+06$ \\
\hline Ba-137 & $1.88 \mathrm{E}+05$ & $1.02 \mathrm{E}+05$ & & $8.65 \mathrm{E}+04$ & $0.00 \mathrm{E}+00$ & & $4.11 \mathrm{E}+06$ & $2.78 \mathrm{E}+06$ \\
\hline Sr-90 & $5.37 \mathrm{E}+02$ & $2.90 \mathrm{E}+02$ & & $2.46 \mathrm{E}+02$ & $0.00 \mathrm{E}+00$ & & $5.29 \mathrm{E}+03$ & $3.57 \mathrm{E}+03$ \\
\hline Y-90 & $5.37 \mathrm{E}+02$ & $2.90 \mathrm{E}+02$ & & $2.46 \mathrm{E}+02$ & $0.00 \mathrm{E}+00$ & & $5.29 \mathrm{E}+03$ & $3.57 \mathrm{E}+03$ \\
\hline Total Curies & $3.88 \mathrm{E}+05$ & $2.10 \mathrm{E}+05$ & & $1.78 \mathrm{E}+05$ & $0.00 \mathrm{E}+00$ & & $8.44 \mathrm{E}+06$ & $5.71 \mathrm{E}+06$ \\
\hline
\end{tabular}

Chemicals (kg)

\begin{tabular}{|c|c|c|c|c|c|c|c|c|}
\hline $\mathrm{Bi}+3$ & & & & & $0.00 \mathrm{E}+00$ & & $1.75 \mathrm{E}+00$ & $1.19 \mathrm{E}+00$ \\
\hline $\mathrm{Ca}+2$ & $2.47 \mathrm{E}-02$ & $1.34 \mathrm{E}-02$ & & $1.13 \mathrm{E}-02$ & $0.00 \mathrm{E}+00$ & & $6.01 \mathrm{E}-01$ & $4.06 \mathrm{E}-01$ \\
\hline$\overline{C s}+$ & $2.29 \mathrm{E}+00$ & $1.24 \mathrm{E}+00$ & & $1.05 \mathrm{E}+00$ & $0.00 \mathrm{E}+00$ & & $4.99 \mathrm{E}+01$ & $3.38 \mathrm{E}+01$ \\
\hline $\mathrm{Fe}+3$ & $1.04 \mathrm{E}-02$ & $5.64 \mathrm{E}-03$ & $\because$ & $4.79 \mathrm{E}-03$ & $0.00 \mathrm{E}+00$ & & & \\
\hline $\mathrm{K}+$ & $3.80 \mathrm{E}+02$ & $2.05 \mathrm{E}+02$ & & $1.74 \mathrm{E}+02$ & $0.00 \mathrm{E}+00$ & & $6.95 \mathrm{E}+03$ & $4.70 \mathrm{E}+03$ \\
\hline $\mathrm{Mg}+2$ & & & & & $0.00 \mathrm{E}+00$ & & & \\
\hline $\mathrm{Mn+4}$ & $3.94 \mathrm{E}-01$ & $2.13 \mathrm{E}-01$ & & 1.81E-01 & $0.00 \mathrm{E}+00$ & & $5.50 \mathrm{E}-01$ & $3.71 \mathrm{E}-01$ \\
\hline $\mathrm{Na}+$ & $1.30 \mathrm{E}+04$ & $7.01 \mathrm{E}+03$ & & $5.95 \mathrm{E}+03$ & $0.00 \mathrm{E}+00$ & $5.47 \mathrm{E}+04$ & $2.52 \mathrm{E}+05$ & $1.70 \mathrm{E}+05$ \\
\hline $\mathrm{Ni}+3$ & 1. $10 \mathrm{E}-02$ & $5.94 \mathrm{E}-03$ & & $5.04 \mathrm{E}-03$ & $0.00 \mathrm{E}+00$ & & & \\
\hline $\mathrm{Si}+4$ & $5.26 \mathrm{E}-02$ & $2.85 \mathrm{E}-02$ & & $2.41 \mathrm{E}-02$ & $0.00 \mathrm{E}+00$ & & $5.28 \mathrm{E}+00$ & $3.57 \mathrm{E}+00$ \\
\hline $\mathrm{Sr}+2$ & $9.87 \mathrm{E}-03$ & $5.34 \mathrm{E}-03$ & & $4.53 \mathrm{E}-03$ & $0.00 E+00$ & & $1.73 \mathrm{E}-01$ & $1.17 \mathrm{E}-01$ \\
\hline $\mathrm{UO}^{2}+2$ & & & & & $0.00 \mathrm{E}+00$ & & $9.42 \mathrm{E}+\infty$ & $6.36 \mathrm{E}+00$ \\
\hline $\mathrm{Zr}+4$ & $2.49 \mathrm{E}-01$ & $1.35 \mathrm{E}-01$ & & $1.14 \mathrm{E}-01$ & $0.00 \mathrm{E}+00$ & & $1.64 \mathrm{E}+00$ & $1.11 \mathrm{E}+00$ \\
\hline $\mathrm{Al}(\mathrm{OH}) 4-$ & $4.53 \mathrm{E}+03$ & $2.45 \mathrm{E}+03$ & & $2.08 \mathrm{E}+03$ & $0.00 \mathrm{E}+00$ & & $9.89 \mathrm{E}+04$ & $6.68 \mathrm{E}+04$ \\
\hline C2O4-2 & $5.98 \mathrm{E}+02$ & $3.23 \mathrm{E}+02$ & & $2.74 \mathrm{E}+02$ & $0.00 \mathrm{E}+00$ & & $2.29 \mathrm{E}+04$ & $1.55 \mathrm{E}+04$ \\
\hline $\mathrm{Cl}-$ & $1.71 \mathrm{E}+01$ & $9.25 \mathrm{E}+00$ & & $7.84 \mathrm{E}+00$ & $0.00 \mathrm{E}+00$ & & $3.17 \mathrm{E}+01$ & $2.14 \mathrm{E}+01$ \\
\hline $\mathrm{CO} 3-2$ & $1.67 \mathrm{E}+03$ & $9.03 \mathrm{E}+02$ & & $7.66 \mathrm{E}+02$ & $0.00 \mathrm{E}+00$ & & $7.89 \mathrm{E}+04$ & $5.33 \mathrm{E}+04$ \\
\hline $\mathrm{Cr}(\mathrm{OH}) 4-$ & $1.39 \mathrm{E}+00$ & $7.54 \mathrm{E}-01$ & & $6.39 \mathrm{E}-01$ & $0.00 \mathrm{E}+00$ & . & $1.13 \mathrm{E}+01$ & $7.65 \mathrm{E}+00$ \\
\hline $\mathrm{CrO} 4-2$ & $1.37 \mathrm{E}+02$ & $7.39 \mathrm{E}+01$ & & $6.27 \mathrm{E}+01$ & $0.00 \mathrm{E}+00$ & & $5.50 \mathrm{E}+03$ & $3.72 \mathrm{E}+03$ \\
\hline $\mathrm{F}-$ & $1.48 \mathrm{E}+02$ & $8.02 \mathrm{E}+01$ & & $6.81 \mathrm{E}+01$ & $0.00 \mathrm{E}+00$ & & $3.71 \mathrm{E}+03$ & $2.50 \mathrm{E}+03$ \\
\hline HCO3- & & & & & $0.00 \mathrm{E}+00$ & & & \\
\hline NO2- & $6.01 E+03$ & $3.25 \mathrm{E}+03$ & & $2.76 \mathrm{E}+03$ & $0.00 \mathrm{E}+00$ & & $1.05 \mathrm{E}+05$ & $7.07 \mathrm{E}+04$ \\
\hline NO3- & $6.46 \mathrm{E}+03$ & $3.49 \mathrm{E}+03$ & & $2.96 \mathrm{E}+03$ & $0.00 \mathrm{E}+00$ & & $7.98 \mathrm{E}+04$ & $5.40 \mathrm{E}+04$ \\
\hline OH- & $2.98 \mathrm{E}+03$ & $1.61 E+03$ & & $1.37 \mathrm{E}+03$ & $0.00 \mathrm{E}+00$ & $4.04 \mathrm{E}+04$ & $3.05 \mathrm{E}+04$ & $2.06 \mathrm{E}+04$ \\
\hline PO4-3 & $1.10 \mathrm{E}+02$ & $5.95 \mathrm{E}+01$ & & $5.05 \mathrm{E}+01$ & $0.00 \mathrm{E}+00$ & & $6.04 \mathrm{E}+02$ & $4.08 \mathrm{E}+02$ \\
\hline SO4-2 & $1.54 \mathrm{E}+03$ & $8.34 \mathrm{E}+02$ & & $7.07 \mathrm{E}+02$ & $0.00 \mathrm{E}+00$ & & $6.21 \mathrm{E}+04$ & $4.20 \mathrm{E}+04$ \\
\hline $\mathrm{H} 2 \mathrm{O}$ & $9.97 E+05$ & $5.39 \mathrm{E}+05$ & $3.10 \mathrm{E}+05$ & $7.68 \mathrm{E}+05$ & $1.68 \mathrm{E}+06$ & $9.51 \mathrm{E}+04$ & $1.63 \mathrm{E}+06$ & $1.10 \mathrm{E}+06$ \\
\hline
\end{tabular}




\section{HNF-SD-TWR-AGA-003}

\section{Revision 0}

Table C-1. Alternative 1 Material Balance.

(Sheet 6 of 12)

\begin{tabular}{|l|c|c|c|c|c|c|c|c|}
\hline STREAM NAME & $5 \mathrm{D}$ & $6 \mathrm{D}$ & 7 & 8 & $2 \mathrm{~B}$ & $10 \mathrm{~A}$ & $11 \mathrm{~A}$ & $12 \mathrm{~A}$ \\
\hline SOLID COMPONENTS & & & & & & & & \\
\hline Total Mass Flow $(\mathrm{kg})$ & $8.69 \mathrm{E}+04$ & $2.87 \mathrm{E}+02$ & & $8.66 \mathrm{E}+04$ & $0.00 \mathrm{E}+00$ & & $1.52 \mathrm{E}+05$ & $5.01 \mathrm{E}+02$ \\
\hline
\end{tabular}

Radionuclides (C)

\begin{tabular}{|c|c|c|c|c|c|c|}
\hline Pu-239 & $9.49 \mathrm{E}+02$ & $3.13 \mathrm{E}+00$ & $9.46 \mathrm{E}+02$ & $0.00 \mathrm{E}+00$ & $1.25 \mathrm{E}+03$ & $4.14 \mathrm{E}+00$ \\
\hline Sr-90 & $6.27 \mathrm{E}+06$ & $2.07 \mathrm{E}+04$ & $6.25 \mathrm{E}+06$ & $0.00 \mathrm{E}+00$ & $3.69 \mathrm{E}+06$ & $1.22 \mathrm{E}+04$ \\
\hline$Y-90$ & $6.27 \mathrm{E}+06$ & $2.07 \mathrm{E}+04$ & $6.25 \mathrm{E}+06$ & $0.00 \mathrm{E}+00$ & $3.69 \mathrm{E}+06$ & $1.22 \mathrm{E}+04$ \\
\hline Total Curies & $1.26 \mathrm{E}+07$ & $4.14 \mathrm{E}+04$ & $1.25 \mathrm{E}+07$ & $0.00 \mathrm{E}+00$ & $7.38 \mathrm{E}+06$ & $2.44 \mathrm{E}+04$ \\
\hline
\end{tabular}

Chemicals (kg)

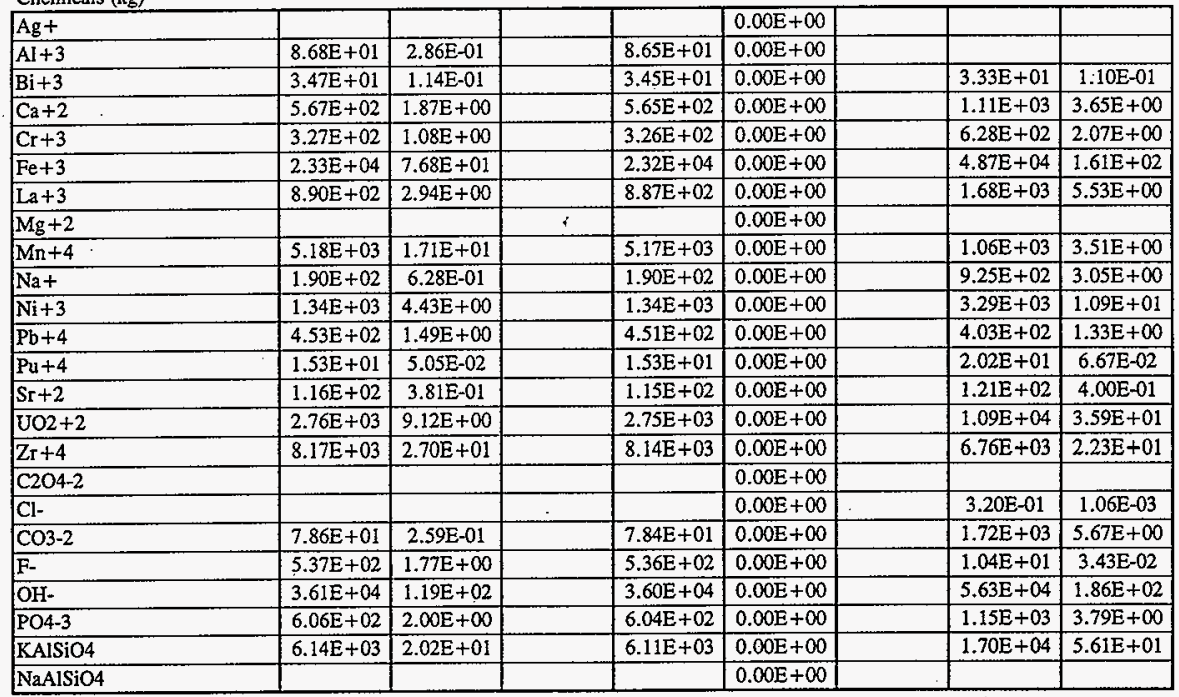


HNF-SD-TWR-AGA-003

Revision 0

Table C-1. Alternative 1 Material Balance.

(Sheet 7 of 12)

\begin{tabular}{|l|c|c|c|c|c|c|c|c|}
\hline $\begin{array}{l}\text { STREAM NAME } \\
\text { LIQUID COMPONENTS }\end{array}$ & $10 \mathrm{~B}$ & $11 \mathrm{~B}$ & $12 \mathrm{~B}$ & $10 \mathrm{C}$ & $11 \mathrm{C}$ & $12 \mathrm{C}$ & 13 & 14 \\
\hline Total Mass Flow (kg) & $2.42 \mathrm{E}+06$ & $3.19 \mathrm{E}+06$ & $2.46 \mathrm{E}+06$ & $2.42 \mathrm{E}+06$ & $3.16 \mathrm{E}+06$ & $2.43 \mathrm{E}+06$ & $6.05 \mathrm{E}+05$ & $1.31 \mathrm{E}+06$ \\
\hline Volume (L) & $2.41 \mathrm{E}+06$ & $3.00 \mathrm{E}+06$ & $2.30 \mathrm{E}+06$ & $2.41 \mathrm{E}+06$ & $3.10 \mathrm{E}+06$ & $2.39 \mathrm{E}+06$ & $3.93 \mathrm{E}+05$ & $1.30 \mathrm{E}+06$ \\
\hline Volume (gal) & $6.38 \mathrm{E}+05$ & $7.91 \mathrm{E}+05$ & $6.09 \mathrm{E}+05$ & $6.38 \mathrm{E}+05$ & $8.20 \mathrm{E}+05$ & $6.31 \mathrm{E}+05$ & $1.04 \mathrm{E}+05$ & $3.43 \mathrm{E}+05$ \\
\hline Specific Gravity & $1.00 \mathrm{E}+00$ & $1.07 \mathrm{E}+00$ & $1.07 \mathrm{E}+00$ & $1.00 \mathrm{E}+00$ & $1.02 \mathrm{E}+00$ & $1.02 \mathrm{E}+00$ & $4.62 \mathrm{E}+00$ & $1.01 \mathrm{E}+00$ \\
\hline
\end{tabular}

Radionuclides (Ci)

\begin{tabular}{|l|l|l|l|l|l|l|l|l|}
\hline Cs-137 & & $1.40 \mathrm{E}+06$ & $1.08 \mathrm{E}+06$ & & $3.23 \mathrm{E}+05$ & $2.49 \mathrm{E}+05$ & $0.00 \mathrm{E}+00$ & $7.46 \mathrm{E}+04$ \\
\hline Ba-137 & & $1.33 \mathrm{E}+06$ & $1.03 \mathrm{E}+06$ & & $3.07 \mathrm{E}+05$ & $2.36 \mathrm{E}+05$ & $0.00 \mathrm{E}+00$ & $7.09 \mathrm{E}+04$ \\
\hline Sr-90 & & $1.71 \mathrm{E}+03$ & $1.32 \mathrm{E}+03$ & & $3.95 \mathrm{E}+02$ & $3.04 \mathrm{E}+02$ & $0.00 \mathrm{E}+00$ & $9.12 \mathrm{E}+01$ \\
\hline Y-90 & & $1.71 \mathrm{E}+03$ & $1.32 \mathrm{E}+03$ & & $3.95 \mathrm{E}+02$ & $3.04 \mathrm{E}+02$ & $0.00 \mathrm{E}+00$ & $9.12 \mathrm{E}+01$ \\
\hline Total Curies & & $2.74 \mathrm{E}+06$ & $2.11 \mathrm{E}+06$ & & $6.32 \mathrm{E}+05$ & $4.86 \mathrm{E}+05$ & $0.00 \mathrm{E}+00$ & $1.46 \mathrm{E}+05$ \\
\hline
\end{tabular}

Chemicals (kg)

\begin{tabular}{|c|c|c|c|c|c|c|c|c|}
\hline $\mathrm{Bi}+3$ & & $5.69 \mathrm{E}-01$ & $4.38 \mathrm{E}-01$ & & $1.31 \mathrm{E}-01$ & $1.01 \mathrm{E}-01$ & $0.00 \mathrm{E}+00$ & $3.03 \mathrm{E}-02$ \\
\hline$\overline{\mathrm{Ca}+2}$ & & $1.95 \mathrm{E}-01$ & $1.50 \mathrm{E}-01$ & & $4.50 \mathrm{E}-02$ & $3.46 \mathrm{E}-02$ & $0.00 \mathrm{E}+00$ & $1.04 \mathrm{E}-02$ \\
\hline Cst & & $1.62 \mathrm{E}+01$ & $1.25 \mathrm{E}+01$ & & $3.74 \mathrm{E}+00$ & $2.87 \mathrm{E}+00$ & $0.00 \mathrm{E}+00$ & $8.61 \mathrm{E}-01$ \\
\hline \multicolumn{9}{|l|}{$\mathrm{Fe}+3$} \\
\hline $\mathrm{K}+$ & & $2.25 \mathrm{E}+03$ & $1.73 E+03$ & & $5.20 \mathrm{E}+02$ & $4.00 \mathrm{E}+02$ & $0.00 \mathrm{E}+00$ & $1.20 \mathrm{E}+02$ \\
\hline $\mathrm{Mg}+2$ & & & & & & & $0.00 \mathrm{E}+00$ & \\
\hline $\mathrm{Mn}+4$ & & $1.78 \mathrm{E}-01$ & $1.37 \mathrm{E}-01$ & & $4.11 \mathrm{E}-02$ & $3.16 \mathrm{E}-02$ & $0.00 \mathrm{E}+00$ & $9.48 \mathrm{E}-03$ \\
\hline $\mathrm{Na}+$ & $6.10 \mathrm{E}+03$ & $8.79 \mathrm{E}+04$ & $6.76 \mathrm{E}+04$ & $6.10 \mathrm{E}+03$ & $2.64 \mathrm{E}+04$ & $2.03 \mathrm{E}+04$ & $1.74 \mathrm{E}+05$ & $6.08 \mathrm{E}+03$ \\
\hline \multicolumn{9}{|l|}{$\mathrm{Ni}+3$} \\
\hline Si+4 & & $1.71 \mathrm{E}+00$ & $1.32 \mathrm{E}+00$ & & $3.95 \mathrm{E}-01$ & $3.04 \mathrm{E}-01$ & $0.00 \mathrm{E}+00$ & $9.11 \mathrm{E}-02$ \\
\hline $\mathrm{Sr}+2$ & & $5.61 \mathrm{E}-02$ & $4.32 \mathrm{E}-02$ & & $1.30 \mathrm{E}-02$ & $9.96 \mathrm{E}-03$ & $0.00 \mathrm{E}+00$ & $2.99 \mathrm{E}-03$ \\
\hline $\mathrm{UO} 2+2$ & & $3.05 \mathrm{E}+00$ & $2.35 \mathrm{E}+\infty$ & & $7.04 E-01$ & $5.42 \mathrm{E}-01$ & $0.00 \mathrm{E}+00$ & $1.62 \mathrm{E}-01$ \\
\hline $\mathrm{Zr}+4$ & & $5.32 \mathrm{E}-01$ & $4.09 \mathrm{E}-01$ & & $1.23 \mathrm{E}-01$ & $9.45 \mathrm{E}-02$ & $0.00 \mathrm{E}+00$ & $2.83 \mathrm{E}-02$ \\
\hline $\mathrm{Al}(\mathrm{OH}) 4-$ & & $3.21 \mathrm{E}+04$ & $2.47 \mathrm{E}+04$ & & $7.40 \mathrm{E}+03$ & $5.69 \mathrm{E}+03$ & $0.00 \mathrm{E}+00$ & $1.71 \mathrm{E}+03$ \\
\hline C2O4-2 & & $7.43 \mathrm{E}+03$ & $5.71 \mathrm{E}+03$ & & $1.71 \mathrm{E}+03$ & $1.32 \mathrm{E}+03$ & $0.00 \mathrm{E}+00$ & $3.95 \mathrm{E}+02$ \\
\hline $\mathrm{Cl}-$ & & $1.03 \mathrm{E}+01$ & $7.91 \mathrm{E}+00$ & & $2.37 \mathrm{E}+00$ & $1.82 \mathrm{E}+00$ & $0.00 \mathrm{E}+00$ & $5.47 \mathrm{E}-01$ \\
\hline $\mathrm{CO} 3-2$ & & $2.56 \mathrm{E}+04$ & $1.97 \mathrm{E}+04$ & & $5.90 \mathrm{E}+03$ & $4.54 \mathrm{E}+03$ & $0.00 \mathrm{E}+00$ & $1.36 \mathrm{E}+03$ \\
\hline $\mathrm{Cr}(\mathrm{OH}) 4-$ & & $3.67 \mathrm{E}+00$ & $2.82 \mathrm{E}+00$ & & $8.46 \mathrm{E}-01$ & $6.51 \mathrm{E}-01$ & $0.00 \mathrm{E}+00$ & $1.95 \mathrm{E}-01$ \\
\hline CrO4-2 & & $1.78 \mathrm{E}+03$ & $1.37 \mathrm{E}+03$ & & $4.12 \mathrm{E}+02$ & $3.17 \mathrm{E}+02$ & $0.00 \mathrm{E}+00$ & $9.49 \mathrm{E}+01$ \\
\hline F. & & $1.20 \mathrm{E}+03$ & $9.24 \mathrm{E}+02$ & & $2.77 E+02$ & $2.13 \mathrm{E}+02$ & $0.00 \mathrm{E}+00$ & $6.39 \mathrm{E}+01$ \\
\hline $\mathrm{HCO} 3-$ & & & & & & & $0.00 \mathrm{E}+00$ & \\
\hline NO2- & $1.11 \mathrm{E}+03$ & $3.50 \mathrm{E}+04$ & $2.69 E+04$ & $1.11 \mathrm{E}+03$ & $9.19 \mathrm{E}+03$ & $7.07 \mathrm{E}+03$ & $1.38 \mathrm{E}-13$ & $2.12 \mathrm{E}+03$ \\
\hline NO3- & & $2.59 \mathrm{E}+04$ & $1.99 \mathrm{E}+04$ & & $5.97 \mathrm{E}+03$ & $4.59 \mathrm{E}+03$ & $0.00 \mathrm{E}+00$ & $1.38 \mathrm{E}+03$ \\
\hline $\mathrm{OH}-$ & $4.11 \mathrm{E}+03$ & $1.40 \mathrm{E}+04$ & $1.08 \mathrm{E}+04$ & $4.11 \mathrm{E}+03$ & $7.33 \mathrm{E}+03$ & $5.64 \mathrm{E}+03$ & $1.29 \mathrm{E}+05$ & $1.69 \mathrm{E}+03$ \\
\hline PO4-3 & & $1.96 \mathrm{E}+02$ & $1.51 \mathrm{E}+02$ & & $4.52 \mathrm{E}+01$ & $3.48 \mathrm{E}+01$ & $0.00 \mathrm{E}+00$ & $1.04 \mathrm{E}+01$ \\
\hline SO4-2 & & $2.01 E+04$ & $1.55 \mathrm{E}+04$ & . & $4.65 \mathrm{E}+03$ & $3.57 \mathrm{E}+03$ & $0.00 \mathrm{E}+00$ & $1.07 \mathrm{E}+03$ \\
\hline $\mathrm{H} 2 \mathrm{O}$ & $2.41 E+06$ & $2.94 \mathrm{E}+06$ & $2.26 \mathrm{E}+06$ & $2.41 \mathrm{E}+06$ & $3.09 \mathrm{E}+06$ & $2.38 \mathrm{E}+06$ & $3.02 \mathrm{E}+05$ & $1.30 \mathrm{E}+06$ \\
\hline
\end{tabular}


HNF-SD-TWR-AGA-003

Revision 0

Table C-1. Alternative 1 Material Balance.

(Sheet 8 of 12)

\begin{tabular}{|l|c|c|c|c|c|c|c|c|}
\hline STREAM NAME & $10 \mathrm{~B}$ & $11 \mathrm{~B}$ & $12 \mathrm{~B}$ & $10 \mathrm{C}$ & $11 \mathrm{C}$ & $12 \mathrm{C}$ & 13 & 14 \\
\hline SOLDD COMPONENTS & & & & & & & & \\
\hline Total Mass Flow (kg) & & $1.51 \mathrm{E}+05$ & $4.99 \mathrm{E}+02$ & & $1.51 \mathrm{E}+05$ & $4.98 \mathrm{E}+02$ & $0.00 \mathrm{E}+00$ & $1.50 \mathrm{E}+05$ \\
\hline
\end{tabular}

Radionuclides (Ci)

\begin{tabular}{|l|l|l|l|l|l|l|l|l|}
\hline Pu-239 & & $1.25 \mathrm{E}+03$ & $4.12 \mathrm{E}+00$ & & $1.25 \mathrm{E}+03$ & $4.11 \mathrm{E}+00$ & $0.00 \mathrm{E}+00$ & $1.24 \mathrm{E}+03$ \\
\hline Sr-90 & & $3.68 \mathrm{E}+06$ & $1.21 \mathrm{E}+04$ & & $3.67 \mathrm{E}+06$ & $1.21 \mathrm{E}+04$ & $0.00 \mathrm{E}+00$ & $3.66 \mathrm{E}+06$ \\
\hline $\mathrm{Y}-90$ & & $3.68 \mathrm{E}+06$ & $1.21 \mathrm{E}+04$ & & $3.67 \mathrm{E}+06$ & $1.21 \mathrm{E}+04$ & $0.00 \mathrm{E}+00$ & $3.66 \mathrm{E}+06$ \\
\hline Total Curies & & $7.36 \mathrm{E}+06$ & $2.43 \mathrm{E}+04$ & & $7.34 \mathrm{E}+06$ & $2.42 \mathrm{E}+04$ & $0.00 \mathrm{E}+00$ & $7.31 \mathrm{E}+06$ \\
\hline
\end{tabular}

Chemicals $(\mathrm{kg})$

\begin{tabular}{|c|c|c|c|c|c|c|}
\hline $\mathrm{Ag}+$ & & & & & $0.00 \mathrm{E}+\infty 0$ & . \\
\hline \multicolumn{7}{|l|}{$\mathrm{Al}+3$} \\
\hline $\mathrm{Bi}+3$ & $3.32 \mathrm{E}+01$ & $1.09 \mathrm{E}-01$ & $3.30 \mathrm{E}+01$ & $1.09 \mathrm{E}-01$ & $0.00 \mathrm{E}+00$ & $3.29 \mathrm{E}+01$ \\
\hline $\mathrm{Ca}+2$ & $1.10 \mathrm{E}+03$ & $3.64 \mathrm{E}+00$ & $1.10 \mathrm{E}+03$ & $3.63 \mathrm{E}+00$ & $0.00 \mathrm{E}+\infty 0$ & $1.10 \mathrm{E}+03$ \\
\hline $\mathrm{Cr}+3$ & $6.26 \mathrm{E}+02$ & $2.07 \mathrm{E}+00$ & $6.24 \mathrm{E}+02$ & $2.06 \mathrm{E}+00$ & $0.00 \mathrm{E}+00$ & $6.22 \mathrm{E}+02$ \\
\hline $\mathrm{Fe}+3$ & $4.86 \mathrm{E}+04$ & $1.60 \mathrm{E}+02$ & $4.84 \mathrm{E}+04$ & $1.60 \mathrm{E}+02$ & $0.00 \mathrm{E}+00$ & $4.83 \mathrm{E}+04$ \\
\hline $\mathrm{La}+3$ & $1.67 \mathrm{E}+03$ & $5.51 \mathrm{E}+\infty 0$ & $1.67 \mathrm{E}+03$ & $5.50 \mathrm{E}+00$ & $0.00 \mathrm{E}+00$ & $1.66 \mathrm{E}+03$ \\
\hline $\mathrm{Mg}+2$ & & 5 & & & $0.00 \mathrm{E}+00$ & \\
\hline$M n+4$ & $1.06 \mathrm{E}+03$ & $3.50 \mathrm{E}+00$ & $1.06 \mathrm{E}+03$ & $3.48 \mathrm{E}+00$ & $0.00 \mathrm{E}+00$ & $1.05 \mathrm{E}+03$ \\
\hline $\mathrm{Na}+$ & $9.22 \mathrm{E}+02$ & $3.04 \mathrm{E}+\infty$ & $9.19 \mathrm{E}+02$ & $3.03 \mathrm{E}+00$ & $0.00 \mathrm{E}+00$ & $9.16 \mathrm{E}+02$ \\
\hline $\mathrm{Ni}+3$ & $3.28 \mathrm{E}+03$ & $1.08 \mathrm{E}+01$ & $3.27 \mathrm{E}+03$ & $1.08 \mathrm{E}+01$ & $0.00 \mathrm{E}+00$ & $3.26 \mathrm{E}+03$ \\
\hline $\mathrm{Pb}+4$ & $4.01 \mathrm{E}+02$ & $1.32 \mathrm{E}+00$ & $4.00 \mathrm{E}+02$ & $1.32 \mathrm{E}+00$ & $0.00 \mathrm{E}+00$ & $3.99 \mathrm{E}+02$ \\
\hline $\mathrm{Pu}+4$ & $2.02 \mathrm{E}+01$ & $6.65 E-02$ & $2.01 E+01$ & $6.63 \mathrm{E}-02$ & $0.00 \mathrm{E}+00$ & $2.00 \mathrm{E}+01$ \\
\hline$S r+2$ & $1.21 \mathrm{E}+02$ & $3.99 E-01$ & 1.20E+02 & $3.97 \mathrm{E}-01$ & $0.00 \mathrm{E}+00$ & $1.20 \mathrm{E}+02$ \\
\hline $\mathrm{UO} 2+2$ & $1.09 \mathrm{E}+04$ & $3.58 \mathrm{E}+01$ & $1.08 \mathrm{E}+04$ & $3.57 \mathrm{E}+01$ & $0.00 \mathrm{E}+00$ & $1.08 \mathrm{E}+04$ \\
\hline$\overline{\mathrm{Zr}+4}$ & $6.73 \mathrm{E}+03$ & $2.22 \mathrm{E}+01$ & $6.71 \mathrm{E}+03$ & $2.22 \mathrm{E}+01$ & $0.00 \mathrm{E}+00$ & $6.69 \mathrm{E}+03$ \\
\hline \multicolumn{7}{|l|}{ C2O4-2 } \\
\hline Ci- & $3.19 \mathrm{E}-01$ & $1.05 \mathrm{E}-03$ & $3.18 \mathrm{E}-01$ & $1.05 \mathrm{E}-03$ & $0.00 \mathrm{E}+00$ & $3.17 \mathrm{E}-01$ \\
\hline $\mathrm{CO} 3-2$ & $1.71 \mathrm{E}+03$ & $5.65 \mathrm{E}+00$ & $1.71 \mathrm{E}+03$ & $5.63 \mathrm{E}+00$ & $0.00 \mathrm{E}+00$ & $1.70 \mathrm{E}+03$ \\
\hline F. & $1.04 \mathrm{E}+01$ & $3.42 \mathrm{E}-02$ & $1.03 \mathrm{E}+01$ & $3.41 \mathrm{E}-02$ & $0.00 \mathrm{E}+00$ & $1.03 \mathrm{E}+01$ \\
\hline OH- & $5.61 \mathrm{E}+04$ & $1.85 \mathrm{E}+02$ & $5.59 \mathrm{E}+04$ & $1.85 \mathrm{E}+02$ & $0.00 \mathrm{E}+00$ & $5.57 \mathrm{E}+04$ \\
\hline PO4-3 & 1. $15 E+03$ & $3.78 \mathrm{E}+00$ & $1.14 E+03$ & $3.77 E+00$ & $0.00 \mathrm{E}+00$ & $1.14 \mathrm{E}+03$ \\
\hline KAlSiO4 & $1.69 E+04$ & $5.59 \mathrm{E}+01$ & $1.69 \mathrm{E}+04$ & $5.57 \mathrm{E}+01$ & $0.00 \mathrm{E}+00$ & $1.68 \mathrm{E}+04$ \\
\hline $\mathrm{NaAlSiO4}$ & & & . & & $0.00 \mathrm{E}+00$ & \\
\hline
\end{tabular}


HNF-SD-TWR-AGA-003

Revision 0

Table C-1. Alternative 1 Material Balance.

(Sheet 9 of 12)

\begin{tabular}{|l|c|c|c|c|c|c|c|c|}
\hline $\begin{array}{l}\text { STREAM NAME } \\
\text { LIQUTD COMPONENTS }\end{array}$ & $16 \mathrm{~A}$ & $17 \mathrm{~A}$ & $18 \mathrm{~A}$ & $16 \mathrm{~B}$ & $17 \mathrm{~B}$ & $18 \mathrm{~B}$ & $16 \mathrm{C}$ & $17 \mathrm{C}$ \\
\hline Total Mass Flow $(\mathrm{kg})$ & $3.10 \mathrm{E}+05$ & $4.12 \mathrm{E}+06$ & $2.99 \mathrm{E}+06$ & $1.23 \mathrm{E}+06$ & $2.36 \mathrm{E}+06$ & $1.25 \mathrm{E}+06$ & $1.23 \mathrm{E}+06$ & $2.35 \mathrm{E}+06$ \\
\hline Volume (L) & $2.02 \mathrm{E}+05$ & $3.61 \mathrm{E}+06$ & $2.62 \mathrm{E}+06$ & $1.23 \mathrm{E}+06$ & $2.22 \mathrm{E}+06$ & $1.17 \mathrm{E}+06$ & $1.23 \mathrm{E}+06$ & $2.28 \mathrm{E}+06$ \\
\hline Volume (gai) & $5.33 \mathrm{E}+04$ & $9.54 \mathrm{E}+05$ & $6.92 \mathrm{E}+05$ & $3.25 \mathrm{E}+05$ & $5.86 \mathrm{E}+05$ & $3.09 \mathrm{E}+05$ & $3.25 \mathrm{E}+05$ & $6.02 \mathrm{E}+05$ \\
\hline Specific Gravity & $1.54 \mathrm{E}+00$ & $1.14 \mathrm{E}+00$ & $1.14 \mathrm{E}+00$ & $1.00 \mathrm{E}+00$ & $1.07 \mathrm{E}+00$ & $1.07 \mathrm{E}+00$ & $1.00 \mathrm{E}+00$ & $1.03 \mathrm{E}+00$ \\
\hline
\end{tabular}

Radionuclides $(\mathrm{C}$ i $)$

\begin{tabular}{|c|c|c|c|c|c|}
\hline Cs-137 & $5.26 \mathrm{E}+05$ & $3.82 \mathrm{E}+05$ & $1.44 \mathrm{E}+05$ & $7.61 E+04$ & $6.81 \mathrm{E}+04$ \\
\hline $\mathrm{Ba}-137$ & $5.00 E+05$ & $3.63 \mathrm{E}+05$ & $1.37 \mathrm{E}+05$ & $7.23 \mathrm{E}+04$ & $6.47 E+04$ \\
\hline \multicolumn{6}{|l|}{ Sr-90 } \\
\hline $\mathrm{Y}-90$ & & & & & \\
\hline Total Curies & $1.03 \mathrm{E}+06$ & $7.45 \mathrm{E}+05$ & $2.81 \mathrm{E}+05$ & $1.48 \mathrm{E}+05$ & $1.33 \mathrm{E}+05$ \\
\hline
\end{tabular}

Chemicals $(\mathrm{kg})$

\begin{tabular}{|c|c|c|c|c|c|c|c|c|}
\hline \multicolumn{9}{|l|}{$\mathrm{Bi}+3$} \\
\hline $\mathrm{Ca}+2$ & & $3.61 \mathrm{E}+00$ & $2.62 \mathrm{E}+00$ & & $9.90 \mathrm{E}-01$ & $5.22 \mathrm{E}-01$ & & $4.68 \mathrm{E}-01$ \\
\hline Cst & & $6.07 \mathrm{E}+00$ & $4.41 \mathrm{E}+00$ & & $1.67 \mathrm{E}+\infty$ & $8.79 \mathrm{E}-01$ & & $7.86 \mathrm{E}-01$ \\
\hline $\mathrm{Fe}+3$ & & & $:$ & & & & & \\
\hline $\mathrm{K}+$ & & $1.49 \mathrm{E}+03$ & $1.08 \mathrm{E}+03$ & & $4.07 \mathrm{E}+02$ & $2.15 \mathrm{E}+02$ & & $1.92 \mathrm{E}+02$ \\
\hline $\mathrm{Mg}+2$ & & $2.00 \mathrm{E}+00$ & $1.45 \mathrm{E}+00$ & & $5.47 \mathrm{E}-01$ & $2.89 \mathrm{E}-01$ & & $2.58 \mathrm{E}-01$ \\
\hline $\mathrm{Mn}+4$ & & $3.51 \mathrm{E}+01$ & $2.55 \mathrm{E}+01$ & & $9.61 \mathrm{E}+00$ & $5.07 \mathrm{E}+\infty$ & & $4.54 \mathrm{E}+00$ \\
\hline $\mathrm{Na}+$ & $8.92 \mathrm{E}+04$ & $2.67 E+05$ & $1.94 \mathrm{E}+05$ & $3.11 \mathrm{E}+03$ & $7.64 \mathrm{E}+04$ & $4.03 \mathrm{E}+04$ & $3.11 \mathrm{E}+03$ & $3.92 \mathrm{E}+04$ \\
\hline \multicolumn{9}{|l|}{$\mathrm{Ni}+3$} \\
\hline $\mathrm{Si}+4$ & & $3.35 \mathrm{E}+01$ & $2.43 \mathrm{E}+01$ & & $9.19 \mathrm{E}+00$ & $4.85 \mathrm{E}+00$ & & $4.34 \mathrm{E}+\infty 0$ \\
\hline \multicolumn{9}{|l|}{$\mathrm{S}_{\mathrm{r}+2}$} \\
\hline $\mathrm{UO} 2+2$ & & $4.04 E+03$ & $2.94 \mathrm{E}+03$ & & $1.11 \mathrm{E}+03$ & $5.85 \mathrm{E}+02$ & & $5.23 \mathrm{E}+02$ \\
\hline $\mathrm{Zr}+4$ & & $1.82 \mathrm{E}-01$ & $1.32 \mathrm{E}-01$ & & $5.00 \mathrm{E}-02$ & $2.64 \mathrm{E}-02$ & & $2.36 \mathrm{E}-02$ \\
\hline $\mathrm{Al}(\mathrm{OH}) 4-$ & & $7.91 \mathrm{E}+04$ & $5.74 \mathrm{E}+04$ & & $2.17 \mathrm{E}+04$ & $1.14 \mathrm{E}+04$ & & $1.02 \mathrm{E}+04$ \\
\hline C2O4-2 & & $6.57 \mathrm{E}+04$ & $4.77 \mathrm{E}+04$ & & $1.80 \mathrm{E}+04$ & $9.50 \mathrm{E}+03$ & & $8.50 \mathrm{E}+03$ \\
\hline $\mathrm{Cl}-$ & & $1.18 \mathrm{E}+03$ & $8.53 \mathrm{E}+02$ & & $3.22 \mathrm{E}+02$ & $1.70 \mathrm{E}+02$ & & $1.52 \mathrm{E}+02$ \\
\hline CO3-2 & & $1.20 \mathrm{E}+05$ & $8.72 \mathrm{E}+04$ & & $3.29 \mathrm{E}+04$ & $1.74 \mathrm{E}+04$ & & $1.55 \mathrm{E}+04$ \\
\hline $\mathrm{Cr}(\mathrm{OH})^{4-}$ & . & $9.85 \mathrm{E}+00$ & $7.15 \mathrm{E}+00$ & & $2.70 \mathrm{E}+00$ & $1.43 \mathrm{E}+00$ & & $1.28 \mathrm{E}+00$ \\
\hline \multicolumn{9}{|l|}{ CrO4-2 } \\
\hline F- & & $2.71 \mathrm{E}+02$ & $1.97 \mathrm{E}+02$ & & $7.44 \mathrm{E}+01$ & $3.92 \mathrm{E}+01$ & & $3.51 E+01$ \\
\hline $\mathrm{HCO} 3-$ & & $4.07 E+04$ & $2.95 \mathrm{E}+04$ & & $1.12 \mathrm{E}+04$ & $5.89 \mathrm{E}+03$ & & $5.27 \mathrm{E}+03$ \\
\hline NO2- & & $2.40 \mathrm{E}+04$ & $1.74 \mathrm{E}+04$ & $5.66 \mathrm{E}+02$ & $7.15 \mathrm{E}+03$ & $3.77 \mathrm{E}+03$ & $5.66 \mathrm{E}+02$ & $3.94 \mathrm{E}+03$ \\
\hline NO3- & & $2.96 \mathrm{E}+03$ & $2.15 \mathrm{E}+03$ & & $8.10 \mathrm{E}+02$ & $4.27 \mathrm{E}+02$ & & $3.83 \mathrm{E}+02$ \\
\hline OH- & $6.60 \mathrm{E}+04$ & $6.14 \mathrm{E}+04$ & $4.46 \mathrm{E}+04$ & $2.09 \mathrm{E}+03$ & $1.89 \mathrm{E}+04$ & $9.98 \mathrm{E}+03$ & $2.09 E+03$ & $1.10 \mathrm{E}+04$ \\
\hline PO4-3 & & $1.08 \mathrm{E}+04$ & $7.85 \mathrm{E}+03$ & & $2.97 \mathrm{E}+03$ & $1.56 \mathrm{E}+03$ & & $1.40 \mathrm{E}+03$ \\
\hline SO4-2 & & $6.77 \mathrm{E}+03$ & $4.92 \mathrm{E}+03$ & & $1.86 \mathrm{E}+03$ & $9.80 \mathrm{E}+02$ & & $8.77 \mathrm{E}+02$ \\
\hline $\mathrm{H} 2 \mathrm{O}$ & $1.55 \mathrm{E}+05$ & $3.43 \mathrm{E}+06$ & $2.49 \mathrm{E}+06$ & $1.23 \mathrm{E}+06$ & $2.17 \mathrm{E}+06$ & $1.14 \mathrm{E}+06$ & $1.23 \mathrm{E}+06$ & $2.25 \mathrm{E}+06$ \\
\hline
\end{tabular}


HNF-SD-TWR-AGA-003

Revision 0

Table C-1. Alternative 1 Material Balance.

(Sheet 10 of 12)

\begin{tabular}{|l|c|c|c|c|c|c|c|c|}
\hline STREAM NAME & $16 \mathrm{~A}$ & $17 \mathrm{~A}$ & $18 \mathrm{~A}$ & $16 \mathrm{~B}$ & $17 \mathrm{~B}$ & $18 \mathrm{~B}$ & $16 \mathrm{C}$ & $17 \mathrm{C}$ \\
\hline SOLID COMPONENTS & & & & & & & & \\
\hline Total Mass Flow (kg) & & $2.47 \mathrm{E}+05$ & $8.16 \mathrm{E}+02$ & & $2.46 \mathrm{E}+05$ & $8.13 \mathrm{E}+02$ & & $2.46 \mathrm{E}+05$ \\
\hline
\end{tabular}

Radionuclides (Ci)

\begin{tabular}{|l|l|l|l|l|l|l|l|l|}
\hline Pu-239 & & $3.51 \mathrm{E}+03$ & $1.16 \mathrm{E}+01$ & & $3.50 \mathrm{E}+03$ & $1.16 \mathrm{E}+01$ & & $3.49 \mathrm{E}+03$ \\
\hline Sr-90 & & $4.23 \mathrm{E}+06$ & $1.40 \mathrm{E}+04$ & & $4.21 \mathrm{E}+06$ & $1.39 \mathrm{E}+04$ & & $4.20 \mathrm{E}+06$ \\
\hline Y-90 & & $4.23 \mathrm{E}+06$ & $1.40 \mathrm{E}+04$ & & $4.21 \mathrm{E}+06$ & $1.39 \mathrm{E}+04$ & & $4.20 \mathrm{E}+06$ \\
\hline Total Curies & & $8.46 \mathrm{E}+06$ & $2.79 \mathrm{E}+04$ & & $8.43 \mathrm{E}+06$ & $2.78 \mathrm{E}+04$ & &. \\
\hline
\end{tabular}

Chemicals (kg)

\begin{tabular}{|c|c|c|c|c|c|}
\hline$\overline{\mathrm{Ag}+}$ & $2.43 \mathrm{E}+03$ & $8.01 E+00$ & $2.42 \mathrm{E}+03$ & $7.99 \mathrm{E}+00$ & $2.41 \mathrm{E}+03$ \\
\hline \multicolumn{6}{|l|}{$\mathrm{Al}+3$} \\
\hline $\mathrm{Bi}+3$ & $7.30 \mathrm{E}+00$ & $2.41 \mathrm{E}-02$ & $7.28 \mathrm{E}+00$ & $2.40 \mathrm{E}-02$ & $7.25 \mathrm{E}+00$ \\
\hline $\mathrm{Ca}+2$ & $4.75 \mathrm{E}+03$ & $1.57 \mathrm{E}+01$ & $4.74 \mathrm{E}+03$ & $1.56 \mathrm{E}+01$ & $4.72 \mathrm{E}+03$ \\
\hline $\mathrm{Cr}+3$ & $1.02 \mathrm{E}+03$ & $3.38 \mathrm{E}+00$ & $1.02 \mathrm{E}+03$ & $3.37 \mathrm{E}+00$ & $1.02 \mathrm{E}+03$ \\
\hline $\mathrm{Fe}+3$ & $5.88 \mathrm{E}+04$ & $1.94 \mathrm{E}+02$ & $5.86 \mathrm{E}+04$ & $1.94 \mathrm{E}+02$ & $5.85 \mathrm{E}+04$ \\
\hline $\mathrm{La}+3$ & $5.68 \mathrm{E}+02$ & $1.87 \mathrm{E}+00$ & $5.66 \mathrm{E}+02$ & $1.87 \mathrm{E}+00$ & $5.64 \mathrm{E}+02$ \\
\hline $\mathrm{Mg}+2$ & $9.76 \mathrm{E}+02$ & $3.22 \mathrm{E}+00$ & $9.73 \mathrm{E}+02$ & $3.21 \mathrm{E}+00$ & $9.70 \mathrm{E}+02$ \\
\hline $\mathrm{Mn}+4$ & $2.83 E+03$ & $9.35 \mathrm{E}+00$ & $2.83 \mathrm{E}+03$ & $9.32 \mathrm{E}+00$ & $2.82 \mathrm{E}+03$ \\
\hline \multicolumn{6}{|l|}{$\mathrm{Na}+$} \\
\hline $\mathrm{Ni}+3$ & $8.90 \mathrm{E}+02$ & $2.94 \mathrm{E}+00$ & $8.87 \mathrm{E}+02$ & $2.93 \mathrm{E}+00$ & $8.84 \mathrm{E}+02$ \\
\hline $\mathrm{Pb}+4$ & $4.36 \mathrm{E}+03$ & $1.44 \mathrm{E}+01$ & $4.35 \mathrm{E}+03$ & $1.44 \mathrm{E}+01$ & $4.33 \mathrm{E}+03$ \\
\hline $\mathrm{Pu}+4$ & $5.67 \mathrm{E}+01$ & $1.87 \mathrm{E}-01$ & $5.65 \mathrm{E}+01$ & $1.86 \mathrm{E}-01$ & $5.63 \mathrm{E}+01$ \\
\hline $\mathrm{Sr}+2$ & $1.52 \mathrm{E}+02$ & 5.01E-01 & $1.51 \mathrm{E}+02$ & $4.99 \mathrm{E}-01$ & $1.51 \mathrm{E}+02$ \\
\hline \multicolumn{6}{|l|}{$\mathrm{UO} 2+2$} \\
\hline $\mathrm{Zr}+4$ & $5.87 \mathrm{E}+02$ & $1.94 \mathrm{E}+00$ & $5.85 \mathrm{E}+02$ & $1.93 \mathrm{E}+00$ & $5.83 E+02$ \\
\hline \multicolumn{6}{|l|}{$\mathrm{C} 2 \mathrm{O} 4-2$} \\
\hline \multicolumn{6}{|l|}{$\mathrm{Cl}-$} \\
\hline $\mathrm{CO} 3-2$ & $7.70 \mathrm{E}+02$ & $2.54 \mathrm{E}+00$ & $7.67 \mathrm{E}+02$ & $2.53 \mathrm{E}+00$ & $7.65 \mathrm{E}+02$ \\
\hline \multicolumn{6}{|l|}{ F- } \\
\hline $\mathrm{OH}-$ & $6.18 \mathrm{E}+04$ & $2.04 \mathrm{E}+02$ & $6.16 \mathrm{E}+04$ & $2.03 E+02$ & $6.14 \mathrm{E}+04$ \\
\hline PO4-3 & $7.85 \mathrm{E}+03$ & $2.59 \mathrm{E}+0 \mathrm{I}$ & $7.83 \mathrm{E}+03$ & $2.58 \mathrm{E}+01$ & $7.80 \mathrm{E}+03$ \\
\hline KAlSiO4 & $4.15 \mathrm{E}+02$ & $1.37 \mathrm{E}+00$ & $4.13 \mathrm{E}+02$ & $1.36 \mathrm{E}+00$ & $4.12 \mathrm{E}+02$ \\
\hline $\mathrm{NaAlSiO4}$ & $9.89 \mathrm{E}+04$ & $3.26 \mathrm{E}+02$ & $9.86 \mathrm{E}+04$ & $3.25 \mathrm{E}+02$ & $9.82 \mathrm{E}+04$ \\
\hline
\end{tabular}


HNF-SD-TWR-AGA-003

Revision 0

Table C-1. Alternative 1 Material Balance.

(Sheet 11 of 12)

\begin{tabular}{|l|c|c|c|}
\hline $\begin{array}{l}\text { STREAM NAME } \\
\text { LIQUID COMPONENTS }\end{array}$ & $18 \mathrm{C}$ & 19 & 20 \\
\hline Total Mass Flow (kg) & $1.24 \mathrm{E}+06$ & $1.33 \mathrm{E}+06$ & $2.44 \mathrm{E}+06$ \\
\hline Volume (L) & $1.20 \mathrm{E}+06$ & $1.33 \mathrm{E}+06$ & $2.41 \mathrm{E}+06$ \\
\hline Volume (gal) & $3.18 \mathrm{E}+05$ & $3.53 \mathrm{E}+05$ & $6.36 \mathrm{E}+05$ \\
\hline Specific Gravity & $1.03 \mathrm{E}+00$ & $1.00 \mathrm{E}+00$ & $1.01 \mathrm{E}+00$ \\
\hline
\end{tabular}

Radionuclides (Ci)

\begin{tabular}{|l|l|l|l|}
\hline Cs-137 & $3.60 \mathrm{E}+04$ & & $3.21 \mathrm{E}+04$ \\
\hline $\mathrm{Ba}-137$ & $3.42 \mathrm{E}+04$ & & $3.05 \mathrm{E}+04$ \\
\hline $\mathrm{Sr}-90$ & $0.00 \mathrm{E}+00$ & & \\
\hline $\mathrm{Y}-90$ & $0.00 \mathrm{E}+00$ & & \\
\hline Total Curies & $7.01 \mathrm{E}+04$ & & $6.26 \mathrm{E}+04$ \\
\hline
\end{tabular}

Chemicals (kg)

\begin{tabular}{|l|c|c|c|}
\hline $\mathrm{Bi}+3$ & $0.00 \mathrm{E}+00$ & & \\
\hline $\mathrm{Ca}+2$ & $2.47 \mathrm{E}-01$ & & $2.21 \mathrm{E}-01$ \\
\hline $\mathrm{Cs}+$ & $4.15 \mathrm{E}-01$ & & $3.71 \mathrm{E}-01$ \\
\hline $\mathrm{Fe}+3$ & & & $\vdots$ \\
\hline $\mathrm{K}+$ & $1.02 \mathrm{E}+02$ & & $9.07 \mathrm{E}+01$ \\
\hline $\mathrm{Mg}+2$ & $1.37 \mathrm{E}-01$ & & $1.22 \mathrm{E}-01$ \\
\hline $\mathrm{Mn}+4$ & $2.40 \mathrm{E}+00$ & & $2.14 \mathrm{E}+00$ \\
\hline $\mathrm{Na}+$ & $2.07 \mathrm{E}+04$ & & $1.85 \mathrm{E}+04$ \\
\hline $\mathrm{Ni}+3$ & & & \\
\hline $\mathrm{Si}+4$ & $2.29 \mathrm{E}+00$ & & $2.05 \mathrm{E}+00$ \\
\hline $\mathrm{Sr}+2$ & $0.00 \mathrm{E}+00$ & & \\
\hline $\mathrm{UO} 2+2$ & $2.77 \mathrm{E}+02$ & & $2.47 \mathrm{E}+02$ \\
\hline $\mathrm{Zr}+4$ & $1.25 \mathrm{E}-02$ & & $1.11 \mathrm{E}-02$ \\
\hline $\mathrm{Al}(\mathrm{OH}) 4-$ & $5.41 \mathrm{E}+03$ & & $4.83 \mathrm{E}+03$ \\
\hline $\mathrm{C} 2 \mathrm{O} 4-2$ & $4.49 \mathrm{E}+03$ & & $4.01 \mathrm{E}+03$ \\
\hline $\mathrm{Cl}-$ & $8.04 \mathrm{E}+01$ & & $7.18 \mathrm{E}+01$ \\
\hline $\mathrm{CO}-2$ & $8.21 \mathrm{E}+03$ & & $7.33 \mathrm{E}+03$ \\
\hline $\mathrm{Cr}(\mathrm{OH}) 4-$ & $6.74 \mathrm{E}-01$ & & $6.02 \mathrm{E}-01$ \\
\hline $\mathrm{CrO4-2}$ & $0.00 \mathrm{E}+00$ & & \\
\hline $\mathrm{F}-$ & $1.86 \mathrm{E}+01$ & & $1.66 \mathrm{E}+01$ \\
\hline $\mathrm{HCO}-$ & $2.78 \mathrm{E}+03$ & & $2.49 \mathrm{E}+03$ \\
\hline $\mathrm{NO} 2-$ & $2.08 \mathrm{E}+03$ & & $1.86 \mathrm{E}+03$ \\
\hline $\mathrm{NO}-$ & $2.02 \mathrm{E}+02$ & & $1.80 \mathrm{E}+02$ \\
\hline $\mathrm{OH}-$ & $5.82 \mathrm{E}+03$ & & $5.20 \mathrm{E}+03$ \\
\hline $\mathrm{PO}-3$ & $7.40 \mathrm{E}+02$ & & $6.61 \mathrm{E}+02$ \\
\hline $\mathrm{SO} 4-2$ & $4.63 \mathrm{E}+02$ & & $4.14 \mathrm{E}+02$ \\
\hline $\mathrm{H} 2 \mathrm{O}$ & $1.19 \mathrm{E}+06$ & $1.33 \mathrm{E}+06$ & $2.40 \mathrm{E}+06$ \\
\hline & & \\
\hline
\end{tabular}




\section{HNF-SD-TWR-AGA-003}

Revision 0

Table C-1. Alternative 1 Material Balance.

\begin{tabular}{|l|c|c|c|}
\hline STREAM NAME & $18 \mathrm{C}$ & 19 & 20 \\
\hline SOLID COMPONENTS & & & \\
\hline Total Mass Flow $(\mathrm{kg})$ & $8.11 \mathrm{E}+02$ & & $2.45 \mathrm{E}+05$ \\
\hline
\end{tabular}

(Sheet 12 of 12)

Radionuclides (Ci)

\begin{tabular}{|l|l|l|l|}
\hline $\mathrm{Pu}-239$ & $1.15 \mathrm{E}+01$ & & $3.48 \mathrm{E}+03$ \\
\hline $\mathrm{Sr}-90$ & $1.39 \mathrm{E}+04$ & & $4.19 \mathrm{E}+06$ \\
\hline $\mathrm{Y}-90$ & $1.39 \mathrm{E}+04$ & & $4.19 \mathrm{E}+06$ \\
\hline Total Curies & $2.77 \mathrm{E}+04$ & & $8.38 \mathrm{E}+06$ \\
\hline
\end{tabular}

Chemicals (kg)

\begin{tabular}{|l|l|l|l|}
\hline $\mathrm{Ag}+$ & $7.96 \mathrm{E}+00$ & & $2.40 \mathrm{E}+03$ \\
\hline $\mathrm{Al}+3$ & & & \\
\hline $\mathrm{Bi}+3$ & $2.39 \mathrm{E}-02$ & & $7.23 \mathrm{E}+00$ \\
\hline $\mathrm{Ca}+2$ & $1.56 \mathrm{E}+01$ & & $4.71 \mathrm{E}+03$ \\
\hline $\mathrm{Cr}+3$ & $3.36 \mathrm{E}+00$ & & $1.01 \mathrm{E}+03$ \\
\hline $\mathrm{Fe}+3$ & $1.93 \mathrm{E}+02$ & & $5.83 \mathrm{E}+04$ \\
\hline $\mathrm{La}+3$ & $1.86 \mathrm{E}+00$ & & $5.62 \mathrm{E}+02$ \\
\hline $\mathrm{Mg}+2$ & $3.20 \mathrm{E}+00$ & & $9.67 \mathrm{E}+02$ \\
\hline $\mathrm{Mn}+4$ & $9.29 \mathrm{E}+00$ & & $2.81 \mathrm{E}+03$ \\
\hline $\mathrm{Na}+$ & $0.00 \mathrm{E}+00$ & & \\
\hline $\mathrm{Ni}+3$ & $2.92 \mathrm{E}+00$ & & $8.81 \mathrm{E}+02$ \\
\hline $\mathrm{Pb}+4$ & $1.43 \mathrm{E}+01$ & & $4.32 \mathrm{E}+03$ \\
\hline $\mathrm{Pu}+4$ & $1.86 \mathrm{E}-01$ & & $5.61 \mathrm{E}+01$ \\
\hline $\mathrm{Sr}+2$ & $4.98 \mathrm{E}-01$ & & $1.50 \mathrm{E}+02$ \\
\hline $\mathrm{UO} 2+2$ & $0.00 \mathrm{E}+00$ & & \\
\hline $\mathrm{Zr}+4$ & $1.92 \mathrm{E}+00$ & & $5.81 \mathrm{E}+02$ \\
\hline $\mathrm{C} 2 \mathrm{O} 4-2$ & & & \\
\hline $\mathrm{Cl}-$ & $0.00 \mathrm{E}+00$ & & \\
\hline $\mathrm{CO} 3-2$ & $2.52 \mathrm{E}+00$ & & $7.62 \mathrm{E}+02$ \\
\hline $\mathrm{F}-$ & $0.00 \mathrm{E}+00$ & & \\
\hline $\mathrm{OH}-$ & $2.03 \mathrm{E}+02$ & & $6.12 \mathrm{E}+04$ \\
\hline $\mathrm{PO} 4-3$ & $2.58 \mathrm{E}+01$ & & $7.78 \mathrm{E}+03$ \\
\hline $\mathrm{KA} 1 S i \mathrm{-}$ & $1.36 \mathrm{E}+00$ & & $4.11 \mathrm{E}+02$ \\
\hline $\mathrm{NaAlSiO4}$ & $3.24 \mathrm{E}+02$ & & $9.79 \mathrm{E}+04$ \\
\hline & & & \\
\hline
\end{tabular}




\section{Revision 0}

Table C-2. Alternative 2 Material Balance.

(Sheet 1 of 12)

\begin{tabular}{|l|c|c|c|c|c|c|c|c|}
\hline $\begin{array}{l}\text { STREAM NAME } \\
\text { LIQUID COMPONENTS }\end{array}$ & AZ101 & AZ102 & AY102 & 0 & 1 & $2 \mathrm{~A}$ & 3 & $4 \mathrm{~A}$ \\
\hline Total Mass Flow $(\mathrm{kg})$ & $3.88 \mathrm{E}+06$ & $3.79 \mathrm{E}+06$ & $3.71 \mathrm{E}+06$ & & & & $3.10 \mathrm{E}+06$ & $5.55 \mathrm{E}+05$ \\
\hline Volume (L) & $3.04 \mathrm{E}+06$ & $3.39 \mathrm{E}+06$ & $3.42 \mathrm{E}+06$ & & & & $2.43 \mathrm{E}+06$ & $5.53 \mathrm{E}+05$ \\
\hline Volume (gai) & $8.03 \mathrm{E}+05$ & $8.96 \mathrm{E}+05$ & $9.03 \mathrm{E}+05$ & & & & $6.41 \mathrm{E}+05$ & $1.46 \mathrm{E}+05$ \\
\hline Specific Gravity & $1.28 \mathrm{E}+00$ & $1.12 \mathrm{E}+00$ & $1.08 \mathrm{E}+00$ & & & & $1.28 \mathrm{E}+00$ & $1.00 \mathrm{E}+00$ \\
\hline
\end{tabular}

Radionuclides $(\mathbf{C i})$

\begin{tabular}{|l|l|l|l|l|l|l|l|l|}
\hline Cs-137 & $7.43 \mathrm{E}+06$ & $4.33 \mathrm{E}+06$ & $5.26 \mathrm{E}+05$ & & & & $5.93 \mathrm{E}+06$ & \\
\hline Ba-137 & $7.06 \mathrm{E}+06$ & $4.11 \mathrm{E}+06$ & $5.00 \mathrm{E}+05$ & & & & $5.63 \mathrm{E}+06$ & \\
\hline Sr-90 & $2.01 \mathrm{E}+04$ & $5.29 \mathrm{E}+03$ & & & & & $1.60 \mathrm{E}+04$ & \\
\hline Y-90 & $2.01 \mathrm{E}+04$ & $5.29 \mathrm{E}+03$ & & & & & $1.60 \mathrm{E}+04$ & \\
\hline Total Curies & $1.45 \mathrm{E}+07$ & $8.44 \mathrm{E}+06$ & $1.03 \mathrm{E}+06$ & & & & $1.16 \mathrm{E}+07$ & \\
\hline
\end{tabular}

Chemicais (kg)

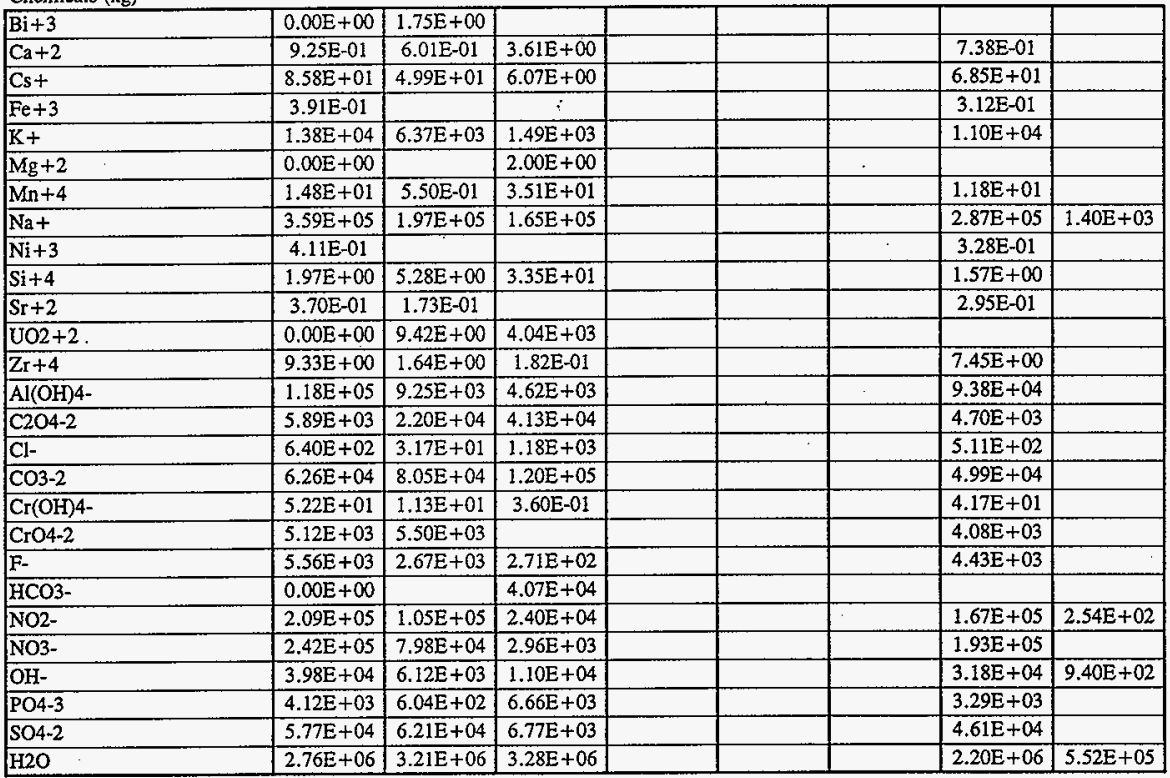




\section{HNF-SD-TWR-AGA-003}

\section{Revision 0}

Table C-2. Alternative 2 Material Balance.

(Sheet 2 of 12)

\begin{tabular}{|l|c|c|c|c|c|c|c|c|}
\hline STREAM NAME & AZ101 & AZ102 & AYl02 & 0. & 1 & $2 A$ & 3 & $4 A$ \\
\hline SOLID COMPONENTS & & & & & & & & \\
\hline Total Mass Flow $(\mathrm{kg})$ & $1.56 \mathrm{E}+05$ & $2.28 \mathrm{E}+05$ & $3.49 \mathrm{E}+05$ & & & & $5.16 \mathrm{E}+02$ & \\
\hline
\end{tabular}

Radionuclides (Ci)

\begin{tabular}{|c|c|c|c|c|c|}
\hline $\mathrm{Pu}-239$ & $9.59 \mathrm{E}+02$ & $1.25 \mathrm{E}+03$ & $3.51 \mathrm{E}+03$ & & $3.16 \mathrm{E}+00$ \\
\hline Sr-90 & $6.34 \mathrm{E}+06$ & $3.69 E+06$ & $4.23 \mathrm{E}+06$ & & $2.09 \mathrm{E}+04$ \\
\hline $\mathrm{X}-90$ & $6.34 \mathrm{E}+06$ & $3.69 \mathrm{E}+06$ & $4.23 \mathrm{E}+06$ & & $2.09 \mathrm{E}+04$ \\
\hline Total Curies & $1.27 \mathrm{E}+07$ & $7.38 \mathrm{E}+06$ & $8.46 \mathrm{E}+06$ & . & $4.18 \mathrm{E}+04$ \\
\hline
\end{tabular}

Chemicals (kg)

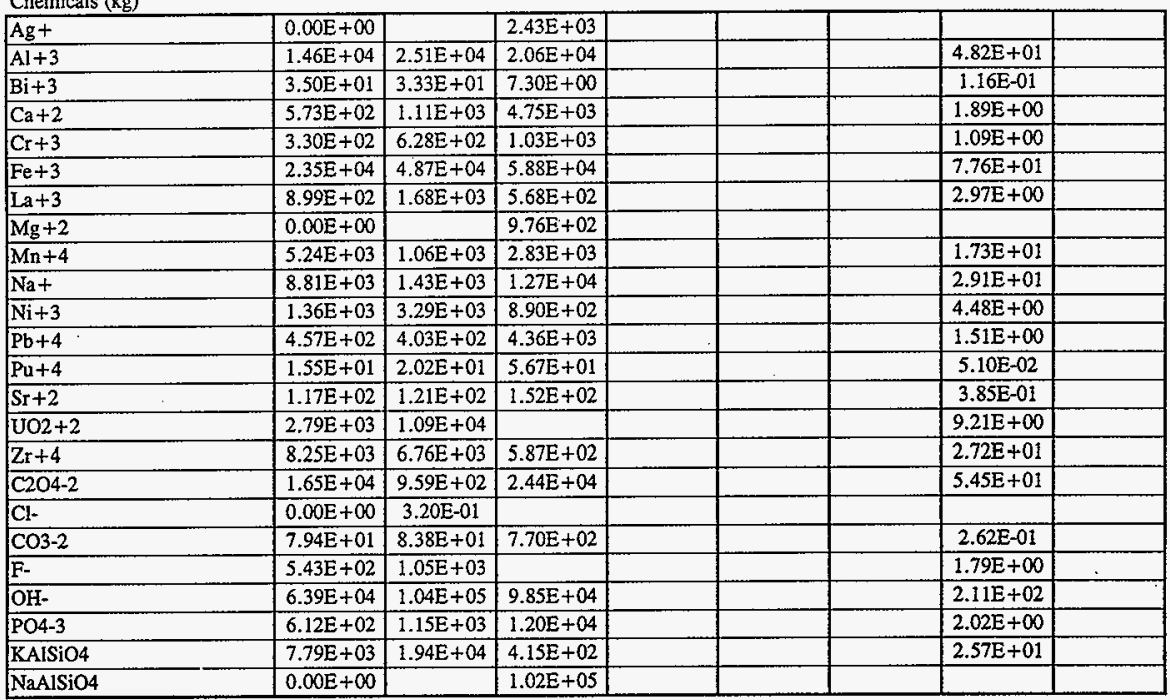


HNF-SD-TWR-AGA-003

Revision 0

Table C-2. Alternative 2 Material Balance.

(Sheet 3 of 12)

\begin{tabular}{|l|c|c|c|c|c|c|c|c|}
\hline $\begin{array}{l}\text { STREAM NAME } \\
\text { LIQUID COMPONENTS }\end{array}$ & $5 \mathrm{~A}$ & $6 \mathrm{~A}$ & $4 \mathrm{~B}$ & $5 \mathrm{~B}$ & $6 \mathrm{~B}$ & $4 \mathrm{C}$ & $5 \mathrm{C}$ & $6 \mathrm{C}$ \\
\hline Total Mass Flow (kg) & $1.36 \mathrm{E}+06$ & $6.97 \mathrm{E}+05$ & $5.55 \mathrm{E}+05$ & $1.22 \mathrm{E}+06$ & $5.66 \mathrm{E}+05$ & $5.55 \mathrm{E}+05$ & $1.21 \mathrm{E}+06$ & $5.61 \mathrm{E}+05$ \\
\hline Volume (L) & $1.18 \mathrm{E}+06$ & $6.03 \mathrm{E}+05$ & $5.53 \mathrm{E}+05$ & $1.13 \mathrm{E}+06$ & $5.24 \mathrm{E}+05$ & $5.53 \mathrm{E}+05$ & $1.16 \mathrm{E}+06$ & $5.38 \mathrm{E}+05$ \\
\hline Volume (gal) & $3.12 \mathrm{E}+05$ & $1.59 \mathrm{E}+05$ & $1.46 \mathrm{E}+05$ & $2.98 \mathrm{E}+05$ & $1.38 \mathrm{E}+05$ & $1.46 \mathrm{E}+05$ & $3.06 \mathrm{E}+05$ & $1.42 \mathrm{E}+05$ \\
\hline Specific Gravity & $1.16 \mathrm{E}+00$ & $1.16 \mathrm{E}+00$ & $1.00 \mathrm{E}+00$ & $1.08 \mathrm{E}+00$ & $1.08 \mathrm{E}+00$ & $1.00 \mathrm{E}+00$ & $1.04 \mathrm{E}+00$ & $1.04 \mathrm{E}+00$ \\
\hline
\end{tabular}

Radionuclides ( $\mathrm{Ci}$ )

\begin{tabular}{|l|l|l|l|l|l|l|l|l|}
\hline Cs-137 & $1.50 \mathrm{E}+06$ & $7.67 \mathrm{E}+05$ & $0.00 \mathrm{E}+00$ & $7.33 \mathrm{E}+05$ & $3.40 \mathrm{E}+05$ & & $3.93 \mathrm{E}+05$ & $1.82 \mathrm{E}+05$ \\
\hline Ba-137 & $1.43 \mathrm{E}+06$ & $7.28 \mathrm{E}+05$ & $0.00 \mathrm{E}+00$ & $6.96 \mathrm{E}+05$ & $3.23 \mathrm{E}+05$ & & $3.74 \mathrm{E}+05$ & $1.73 \mathrm{E}+05$ \\
\hline Sr-90 & $4.06 \mathrm{E}+03$ & $2.07 \mathrm{E}+03$ & $0.00 \mathrm{E}+00$ & $1.98 \mathrm{E}+03$ & $9.19 \mathrm{E}+02$ & & $1.06 \mathrm{E}+03$ & $4.94 \mathrm{E}+02$ \\
\hline Y-90 & $4.06 \mathrm{E}+03$ & $2.07 \mathrm{E}+03$ & $0.00 \mathrm{E}+00$ & $1.98 \mathrm{E}+03$ & $9.19 \mathrm{E}+02$ & & $1.06 \mathrm{E}+03$ & $4.94 \mathrm{E}+02$ \\
\hline Total Curies & $2.93 \mathrm{E}+06$ & $1.50 \mathrm{E}+06$ & $0.00 \mathrm{E}+00$ & $1.43 \mathrm{E}+06$ & $6.64 \mathrm{E}+05$ & & $7.69 \mathrm{E}+05$ & $3.57 \mathrm{E}+05$ \\
\hline
\end{tabular}

Chemicals $(\mathrm{kg})$

\begin{tabular}{|c|c|c|c|c|c|c|c|c|}
\hline$B i+3$ & & & $0.00 \mathrm{E}+00$ & & & & & \\
\hline $\mathrm{Ca}+2$ & $1.87 \mathrm{E}-01$ & $9.54 \mathrm{E}-02$ & $0.00 \mathrm{E}+00$ & $9.12 \mathrm{E}-02$ & $4.23 \mathrm{E}-02$ & & $4.89 \mathrm{E}-02$ & $2.27 \mathrm{E}-02$ \\
\hline Cst & $1.73 \mathrm{E}+01$ & $8.85 \mathrm{E}+\infty 0$ & $0.00 \mathrm{E}+\infty 0$ & $8.46 \mathrm{E}+\infty 0$ & $3.92 \mathrm{E}+00$ & & $4.54 \mathrm{E}+\infty$ & $2.11 \mathrm{E}+00$ \\
\hline $\mathrm{Fe}+3$ & $7.88 \mathrm{E}-02$ & $4.03 \mathrm{E}-02$ & $0.00 \mathrm{E}+00$ & $3.85 \mathrm{E}-02$ & $1.79 \mathrm{E}-02$ & & 2.07E-02 & $9.59 \mathrm{E}-03$ \\
\hline $\mathrm{K}+$ & $2.79 \mathrm{E}+03$ & $1.43 E+03$ & $0.00 \mathrm{E}+00$ & $1.36 \mathrm{E}+03$ & $6.32 \mathrm{E}+02$ & & $7.32 \mathrm{E}+02$ & $3.39 \mathrm{E}+02$ \\
\hline $\mathrm{Mg}+2$ & & & $0.00 \mathrm{E}+00$ & & & & & \\
\hline $\mathrm{Mn}+4$ & $2.98 \mathrm{E}+00$ & $1.53 \mathrm{E}+00$ & $0.00 \mathrm{E}+00$ & $1.46 \mathrm{E}+00$ & $6.75 \mathrm{E}-01$ & & $7.82 \mathrm{E}-01$ & $3.63 \mathrm{E}-01$ \\
\hline $\mathrm{Na}+$ & $8.25 \mathrm{E}+04$ & $4.22 \mathrm{E}+04$ & $1.40 \mathrm{E}+03$ & $4.17 \mathrm{E}+04$ & $1.93 \mathrm{E}+04$ & $1.40 \mathrm{E}+03$ & $2.38 \mathrm{E}+04$ & $1.10 \mathrm{E}+04$ \\
\hline $\mathrm{Ni}+3$ & $8.30 \mathrm{E}-02$ & $4.24 \mathrm{E}-02$ & $0.00 \mathrm{E}+00$ & $4.06 \mathrm{E}-02$ & $1.88 \mathrm{E}-02$ & & $2.18 \mathrm{E}-02$ & $1.01 \mathrm{E}-02$ \\
\hline $\mathrm{Si}+4$ & $3.98 \mathrm{E}-01$ & $2.03 \mathrm{E}-01$ & $0.00 \mathrm{E}+00$ & $1.94 \mathrm{E}-01$ & $9.01 \mathrm{E}-02$ & & $1.04 \mathrm{E}-01$ & $4.84 \mathrm{E}-02$ \\
\hline $\mathrm{Sr}+2$ & $7.46 \mathrm{E}-02$ & $3.82 \mathrm{E}-02$ & $0.00 \mathrm{E}+00$ & $3.65 \mathrm{E}-02$ & $1.69 \mathrm{E}-02$ & & $1.96 \mathrm{E}-02$ & $9.08 \mathrm{E}-03$ \\
\hline $\mathrm{UO} 2+2$ & & & $0.00 \mathrm{E}+00$ & & & & & \\
\hline $\mathrm{Zr+4}$ & $1.88 \mathrm{E}+00$ & $9.63 \mathrm{E}-01$ & $0.00 \mathrm{E}+00$ & $9.21 \mathrm{E}-01$ & $4.27 \mathrm{E}-01$ & & $4.94 \mathrm{E}-01$ & $2.29 \mathrm{E}-01$ \\
\hline $\mathrm{Al}(\mathrm{OH}) 4-$ & $2.37 \mathrm{E}+04$ & $1.21 \mathrm{E}+04$ & $0.00 \mathrm{E}+00$ & $1.16 \mathrm{E}+04$ & $5.37 \mathrm{E}+03$ & & $6.22 \mathrm{E}+03$ & $2.89 \mathrm{E}+03$ \\
\hline $\mathrm{C}_{2} \mathrm{O} 4-2$ & $1.76 \mathrm{E}+04$ & $9.02 \mathrm{E}+03$ & $0.00 \mathrm{E}+00$ & $8.62 \mathrm{E}+03$ & $4.00 \mathrm{E}+03$ & & $4.63 \mathrm{E}+03$ & $2.15 \mathrm{E}+03$ \\
\hline $\mathrm{Cl}-$ & $1.29 \mathrm{E}+02$ & $6.61 \mathrm{E}+01$ & $0.00 \mathrm{E}+00$ & $6.32 \mathrm{E}+01$ & $2.93 \mathrm{E}+01$ & & $3.39 \mathrm{E}+01$ & $1.57 \mathrm{E}+01$ \\
\hline CO3-2 & $1.26 \mathrm{E}+04$ & $6.46 \mathrm{E}+03$ & $0.00 \mathrm{E}+00$ & $6.17 \mathrm{E}+03$ & $2.86 \mathrm{E}+03$ & & $3.31 \mathrm{E}+03$ & $1.54 \mathrm{E}+03$ \\
\hline $\mathrm{Cr}(\mathrm{OH}) 4-$ & $1.05 \mathrm{E}+01$ & $5.39 \mathrm{E}+00$ & $0.00 \mathrm{E}+00$ & $5.15 \mathrm{E}+00$ & $2.39 \mathrm{E}+00$ & & $2.76 \mathrm{E}+00$ & $1.28 \mathrm{E}+00$ \\
\hline $\mathrm{CrO} 4-2$ & $1.03 \mathrm{E}+03$ & $5.28 \mathrm{E}+02$ & $0.00 \mathrm{E}+00$ & $5.05 \mathrm{E}+02$ & $2.34 \overline{\mathrm{E}}+02$ & & $2.71 \mathrm{E}+02$ & $1.26 \mathrm{E}+02$ \\
\hline F- & $1.12 \mathrm{E}+03$ & $5.73 \mathrm{E}+02$ & $0.00 \mathrm{E}+00$ & $5.48 \mathrm{E}+02$ & $2.54 \mathrm{E}+02$ & & $2.94 \mathrm{E}+02$ & $1.36 \mathrm{E}+02$ \\
\hline $\mathrm{HCO}-$ & & & $0.00 \mathrm{E}+00$ & & & & & \\
\hline $\mathrm{NO} 2-$ & $4.25 \mathrm{E}+04$ & $2.17 \mathrm{E}+04$ & $2.54 \mathrm{E}+02$ & $2.10 \mathrm{E}+04$ & $9.73 \overline{\mathrm{E}}+03$ & $2.54 \mathrm{E}+02$ & $1.15 \mathrm{E}+04$ & $5.35 \mathrm{E}+03$ \\
\hline NO3- & $4.88 \mathrm{E}+04$ & $2.50 \mathrm{E}+04$ & $0.00 \mathrm{E}+00$ & $2.39 \mathrm{E}+04$ & $1.11 E+04$ & & $1.28 \mathrm{E}+04$ & $5.94 \mathrm{E}+03$ \\
\hline OH- & $8.98 \mathrm{E}+03$ & $4.59 \mathrm{E}+03$ & $9.40 \mathrm{E}+02$ & $5.33 \mathrm{E}+03$ & $2.47 \mathrm{E}+03$ & $9.40 \mathrm{E}+02$ & $3.80 \mathrm{E}+03$ & $1.76 \mathrm{E}+03$ \\
\hline PO4-3 & $8.32 \mathrm{E}+02$ & $4.25 \mathrm{E}+02$ & $0.00 \mathrm{E}+00$ & $4.07 \mathrm{E}+02$ & $1.88 \mathrm{E}+02$ & & $2.18 \mathrm{E}+02$ & $1.01 \mathrm{E}+02$ \\
\hline SO4-2 & $1.17 \mathrm{E}+04$ & $5.96 \mathrm{E}+03$ & $0.00 \mathrm{E}+00$ & $5.69 \mathrm{E}+03$ & $2.64 \mathrm{E}+03$ & & $3.06 \mathrm{E}+03$ & $1.42 \mathrm{E}+03$ \\
\hline $\mathrm{H} 20$ & $1.11 \mathrm{E}+06$ & $5.67 \mathrm{E}+05$ & $5.52 \mathrm{E}+05$ & $1.09 \mathrm{E}+06$ & $5.07 \mathrm{E}+05$ & $5.52 \mathrm{E}+05$ & $1.14 \mathrm{E}+06$ & $5.28 \mathrm{E}+05$ \\
\hline
\end{tabular}




\section{HNF-SD-TWR-AGA-003 \\ Revision 0}

Table C-2. Alternative 2 Material Balance.

(Sheet 4 of 12)

\begin{tabular}{|l|c|c|c|c|c|c|c|c|}
\hline STREAM NAME & $5 \mathrm{~A}$ & $6 \mathrm{~A}$ & $4 \mathrm{~B}$ & $5 \mathrm{~B}$ & $6 \mathrm{~B}$ & $4 \mathrm{C}$ & $5 \mathrm{C}$ & $6 \mathrm{C}$ \\
\hline SOLID COMPONENTS & & & & & & & & \\
\hline Total Mass Flow $(\mathrm{kg})$ & $1.31 \mathrm{E}+05$ & $4.32 \mathrm{E}+02$ & $0.00 \mathrm{E}+00$ & $1.31 \mathrm{E}+05$ & $4.31 \mathrm{E}+02$ & & $1.30 \mathrm{E}+05$ & $4.29 \mathrm{E}+02$ \\
\hline
\end{tabular}

Radionuclides (Ci)

\begin{tabular}{|l|l|l|l|l|l|l|l|l|}
\hline Pu-239 & $9.56 \mathrm{E}+02$ & $3.15 \mathrm{E}+00$ & $0.00 \mathrm{E}+00$ & $9.53 \mathrm{E}+02$ & $3.14 \mathrm{E}+00$ & & $9.49 \mathrm{E}+02$ & $3.13 \mathrm{E}+00$ \\
\hline St-90 & $6.31 \mathrm{E}+06$ & $2.08 \mathrm{E}+04$ & $0.00 \mathrm{E}+00$ & $6.29 \mathrm{E}+06$ & $2.08 \mathrm{E}+04$ & & $6.27 \mathrm{E}+06$ & $2.07 \mathrm{E}+04$ \\
\hline Y-90 & $6.31 \mathrm{E}+06$ & $2.08 \mathrm{E}+04$ & $0.00 \mathrm{E}+00$ & $6.29 \mathrm{E}+06$ & $2.08 \mathrm{E}+04$ & & $6.27 \mathrm{E}+06$ & $2.07 \mathrm{E}+04$ \\
\hline Total Curies & $1.26 \mathrm{E}+07$ & $4.17 \mathrm{E}+04$ & $0.00 \mathrm{E}+00$ & $1.26 \mathrm{E}+07$ & $4.15 \mathrm{E}+04$ & & $1.26 \mathrm{E}+07$ & $4.14 \mathrm{E}+04$ \\
\hline
\end{tabular}

Chemicals (kg)

\begin{tabular}{|l|c|c|c|c|c|c|c|c|}
\hline $\mathrm{Ag}+$ & & & $0.00 \mathrm{E}+00$ & & & & & \\
\hline $\mathrm{Al}+3$ & $1.46 \mathrm{E}+04$ & $4.80 \mathrm{E}+01$ & $0.00 \mathrm{E}+00$ & $1.45 \mathrm{E}+04$ & $4.79 \mathrm{E}+01$ & & $1.45 \mathrm{E}+04$ & $4.77 \mathrm{E}+01$ \\
\hline $\mathrm{Bi}+3$ & $3.49 \mathrm{E}+01$ & $1.15 \mathrm{E}-01$ & $0.00 \mathrm{E}+00$ & $3.48 \mathrm{E}+01$ & $1.15 \mathrm{E}-01$ & & $3.47 \mathrm{E}+01$ & $1.14 \mathrm{E}-01$ \\
\hline $\mathrm{Ca}+2$ & $5.71 \mathrm{E}+02$ & $1.88 \mathrm{E}+00$ & $0.00 \mathrm{E}+00$ & $5.69 \mathrm{E}+02$ & $1.88 \mathrm{E}+00$ & & $5.67 \mathrm{E}+02$ & $1.87 \mathrm{E}+00$ \\
\hline $\mathrm{Cr}+3$ & $3.29 \mathrm{E}+02$ & $1.09 \mathrm{E}+00$ & $0.00 \mathrm{E}+00$ & $3.28 \mathrm{E}+02$ & $1.08 \mathrm{E}+00$ & & $3.27 \mathrm{E}+02$ & $1.08 \mathrm{E}+00$ \\
\hline $\mathrm{Fe}+3$ & $2.34 \mathrm{E}+04$ & $7.73 \mathrm{E}+01$ & $0.00 \mathrm{E}+00$ & $2.34 \mathrm{E}+04$ & $7.70 \mathrm{E}+01$ & & $2.33 \mathrm{E}+04$ & $7.68 \mathrm{E}+01$ \\
\hline $\mathrm{La}+3$ & $8.96 \mathrm{E}+02$ & $2.96 \mathrm{E}+00$ & $0.00 \mathrm{E}+00$ & $8.93 \mathrm{E}+02$ & $2.95 \mathrm{E}+00$ & & $8.90 \mathrm{E}+02$ & $2.94 \mathrm{E}+00$ \\
\hline $\mathrm{Mg}+2$ & & & $0.00 \mathrm{E}+00$ & & & & & \\
\hline $\mathrm{Mn}+4$ & $5.22 \mathrm{E}+03$ & $1.72 \mathrm{E}+01$ & $0.00 \mathrm{E}+00$ & $5.20 \mathrm{E}+03$ & $1.72 \mathrm{E}+01$ & & $5.18 \mathrm{E}+03$ & $1.71 \mathrm{E}+01$ \\
\hline $\mathrm{Na}+$ & $1.92 \mathrm{E}+02$ & $6.33 \mathrm{E}-01$ & $0.00 \mathrm{E}+00$ & $1.91 \mathrm{E}+02$ & $6.30 \mathrm{E}-01$ & & $1.90 \mathrm{E}+02$ & $6.28 \mathrm{E}-01$ \\
\hline $\mathrm{Ni}+3$ & $1.35 \mathrm{E}+03$ & $4.46 \mathrm{E}+00$ & $0.00 \mathrm{E}+00$ & $1.35 \mathrm{E}+03$ & $4.45 \mathrm{E}+00$ & & $1.34 \mathrm{E}+03$ & $4.43 \mathrm{E}+00$ \\
\hline $\mathrm{Pb}+4$ & $4.56 \mathrm{E}+02$ & $1.50 \mathrm{E}+00$ & $0.00 \mathrm{E}+00$ & $4.54 \mathrm{E}+02$ & $1.50 \mathrm{E}+00$ & & $4.53 \mathrm{E}+02$ & $1.49 \mathrm{E}+00$ \\
\hline $\mathrm{Pu}+4$ & $1.54 \mathrm{E}+01$ & $5.09 \mathrm{E}-02$ & $0.00 \mathrm{E}+00$ & $1.54 \mathrm{E}+01$ & $5.07 \mathrm{E}-02$ & & $1.53 \mathrm{E}+01$ & $5.05 \mathrm{E}-02$ \\
\hline $\mathrm{Sr}+2$ & $1.16 \mathrm{E}+02$ & $3.84 \mathrm{E}-01$ & $0.00 \mathrm{E}+00$ & $1.16 \mathrm{E}+02$ & $3.82 \mathrm{E}-01$ & & $1.16 \mathrm{E}+02$ & $3.81 \mathrm{E}-01$ \\
\hline $\mathrm{UO}+2$ & $2.78 \mathrm{E}+03$ & $9.18 \mathrm{E}+00$ & $0.00 \mathrm{E}+00$ & $2.77 \mathrm{E}+03$ & $9.15 \mathrm{E}+00$ & & $2.76 \mathrm{E}+03$ & $9.12 \mathrm{E}+00$ \\
\hline $\mathrm{Zr}+4$ & $8.22 \mathrm{E}+03$ & $2.71 \mathrm{E}+01$ & $0.00 \mathrm{E}+00$ & $8.20 \mathrm{E}+03$ & $2.71 \mathrm{E}+01$ & & $8.17 \mathrm{E}+03$ & $2.70 \mathrm{E}+01$ \\
\hline $\mathrm{C} 2 \mathrm{O} 4-2$ & & & $0.00 \mathrm{E}+00$ & & & & & \\
\hline $\mathrm{Cl}-$ & & & $0.00 \mathrm{E}+00$ & & & & & \\
\hline $\mathrm{CO} 3-2$ & $7.91 \mathrm{E}+01$ & $2.61 \mathrm{E}-01$ & $0.00 \mathrm{E}+00$ & $7.89 \mathrm{E}+01$ & $2.60 \mathrm{E}-01$ & & $7.86 \mathrm{E}+01$ & $2.59 \mathrm{E}-01$ \\
\hline $\mathrm{F}-$ & $5.41 \mathrm{E}+02$ & $1.79 \mathrm{E}+00$ & $0.00 \mathrm{E}+00$ & $5.39 \mathrm{E}+02$ & $1.78 \mathrm{E}+00$ & & $5.37 \mathrm{E}+02$ & $1.77 \mathrm{E}+00$ \\
\hline $\mathrm{OH}-$ & $6.37 \mathrm{E}+04$ & $2.10 \mathrm{E}+02$ & $0.00 \mathrm{E}+00$ & $6.35 \mathrm{E}+04$ & $2.10 \mathrm{E}+02$ & & $6.33 \mathrm{E}+04$ & $2.09 \mathrm{E}+02$ \\
\hline $\mathrm{PO} 4-3$ & $6.10 \mathrm{E}+02$ & $2.01 \mathrm{E}+00$ & $0.00 \mathrm{E}+00$ & $6.08 \mathrm{E}+02$ & $2.01 \mathrm{E}+00$ & & $6.06 \mathrm{E}+02$ & $2.00 \mathrm{E}+00$ \\
\hline $\mathrm{KAlSiO} 4$ & $7.77 \mathrm{E}+03$ & $2.56 \mathrm{E}+01$ & $0.00 \mathrm{E}+00$ & $7.74 \mathrm{E}+03$ & $2.56 \mathrm{E}+01$ & & $7.72 \mathrm{E}+03$ & $2.55 \mathrm{E}+01$ \\
\hline $\mathrm{NaAlSiO} 4$ & & & $0.00 \mathrm{E}+00$ & & & & & \\
\hline
\end{tabular}


HNF-SD-TWR-AGA-003

Revision 0

Table C-2. Alternative 2 Material Balance.

(Sheet 5 of 12)

\begin{tabular}{|l|c|c|c|c|c|c|c|c|}
\hline $\begin{array}{l}\text { STREAM NAME } \\
\text { LIQUID COMPONENTS }\end{array}$ & 7 & 8 & $2 \mathrm{~B}$ & 9 & $10 \mathrm{~A}$ & $11 \mathrm{~A}$ & $12 \mathrm{~A}$ & $10 \mathrm{~B}$ \\
\hline Total Mass Flow (kg) & $5.46 \mathrm{E}+05$ & $1.20 \mathrm{E}+06$ & $1.68 \mathrm{E}+06$ & $1.62 \mathrm{E}+06$ & $8.07 \mathrm{E}+05$ & $1.30 \mathrm{E}+06$ & $2.70 \mathrm{E}+05$ & $8.07 \mathrm{E}+05$ \\
\hline Volume (L) & $5.46 \mathrm{E}+05$ & $1.17 \mathrm{E}+06$ & $1.68 \mathrm{E}+06$ & $1.31 \mathrm{E}+06$ & $8.05 \mathrm{E}+05$ & $1.21 \mathrm{E}+06$ & $2.50 \mathrm{E}+05$ & $8.05 \mathrm{E}+05$ \\
\hline Volume (gal) & $1.44 \mathrm{E}+05$ & $3.09 \mathrm{E}+05$ & $4.44 \mathrm{E}+05$ & $3.47 \mathrm{E}+05$ & $2.13 \mathrm{E}+05$ & $3.19 \mathrm{E}+05$ & $6.60 \mathrm{E}+04$ & $2.13 \mathrm{E}+05$ \\
\hline Specific Gravity & $1.00 \mathrm{E}+00$ & $1.02 \mathrm{E}+00$ & $1.00 \mathrm{E}+00$ & $1.23 \mathrm{E}+00$ & $1.00 \mathrm{E}+00$ & $1.08 \mathrm{E}+00$ & $1.08 \mathrm{E}+00$ & $1.00 \mathrm{E}+00$ \\
\hline
\end{tabular}

Radionuclides (Ci)

\begin{tabular}{|l|l|l|l|l|l|l|l|l|}
\hline Cs-137 & & $2.11 \mathrm{E}+05$ & & $3.31 \mathrm{E}+06$ & $0.00 \mathrm{E}+00$ & $1.01 \mathrm{E}+06$ & $2.09 \mathrm{E}+05$ & \\
\hline $\mathrm{Ba}-137$ & & $2.00 \mathrm{E}+05$ & & $3.15 \mathrm{E}+06$ & $0.00 \mathrm{E}+00$ & $9.61 \mathrm{E}+05$ & $1.99 \mathrm{E}+05$ & \\
\hline $\mathrm{Sr}-90$ & & $5.71 \mathrm{E}+02$ & & $4.05 \mathrm{E}+03$ & $0.00 \mathrm{E}+00$ & $1.24 \mathrm{E}+03$ & $2.56 \mathrm{E}+02$ & \\
\hline $\mathrm{Y}-90$ & & $5.71 \mathrm{E}+02$ & & $4.05 \mathrm{E}+03$ & $0.00 \mathrm{E}+00$ & $1.24 \mathrm{E}+03$ & $2.56 \mathrm{E}+02$ & \\
\hline Total Curies & & $4.12 \mathrm{E}+05$ & & $6.47 \mathrm{E}+06$ & $0.00 \mathrm{E}+00$ & $1.98 \mathrm{E}+06$ & $4.09 \mathrm{E}+05$ & \\
\hline
\end{tabular}

Chemicals $(\mathrm{kg})$

\begin{tabular}{|c|c|c|c|c|c|c|c|c|}
\hline $\mathrm{Bi}+3$ & & & & $1.34 \mathrm{E}+\infty 0$ & $0.00 \mathrm{E}+00$ & $4.10 \mathrm{E}-01$ & $8.49 \mathrm{E}-02$ & \\
\hline $\mathrm{Ca}+2$ & & $2.62 \mathrm{E}-02$ & & $4.61 \mathrm{E}-01$ & $0.00 \mathrm{E}+\infty$ & $1.41 \mathrm{E}-01$ & $2.91 \mathrm{E}-02$ & \\
\hline Cst & & $2.44 \mathrm{E}+00$ & & $3.83 \mathrm{E}+01$ & $0.00 \mathrm{E}+00$ & $1.17 \mathrm{E}+01$ & $2.42 \mathrm{E}+00$ & \\
\hline $\mathrm{Fe}+3$ & & $1.11 \mathrm{E}-02$ & $i$ & & $0.00 \mathrm{E}+00$ & & & \\
\hline $\mathrm{K}+$ & & $3.92 \mathrm{E}+02$ & & $4.88 \mathrm{E}+03$ & $0.00 \mathrm{E}+00$ & $1.49 \mathrm{E}+03$ & $3.08 \mathrm{E}+02$ & \\
\hline $\mathrm{Mg}+2$ & & & & & $0.00 \mathrm{E}+00$ & & & \\
\hline $\mathrm{Mn}+4$ & & $4.19 \mathrm{E}-01$ & & $4.21 \mathrm{E}-01$ & $0.00 \mathrm{E}+\infty$ & $1.29 \mathrm{E}-01$ & $2.66 \mathrm{E}-02$ & \\
\hline $\mathrm{Na}+$ & & $1.28 \mathrm{E}+04$ & & $1.51 \mathrm{E}+05$ & $2.04 \mathrm{E}+03$ & $4.86 \mathrm{E}+04$ & $1.01 \mathrm{E}+04$ & $2.04 \mathrm{E}+03$ \\
\hline $\mathrm{Ni}+3$ & & $1.17 \mathrm{E}-02$ & & & $0.00 \mathrm{E}+00$ & & & \\
\hline $\mathrm{Si+4}$ & & $5.59 \mathrm{E}-02$ & & $4.04 \mathrm{E}+00$ & $0.00 \mathrm{E}+00$ & $1.24 \mathrm{E}+00$ & $2.56 \mathrm{E}-01$ & \\
\hline $\mathrm{Sr}+2$ & & $1.05 \mathrm{E}-02$ & & $1.33 \mathrm{E}-01$ & $0.00 \mathrm{E}+00$ & $4.05 \mathrm{E}-02$ & $8.38 \mathrm{E}-03$ & \\
\hline $\mathrm{UO} 2+2$ & & & & $7.22 E+00$ & $0.00 \mathrm{E}+00$ & $2.20 \mathrm{E}+00$ & $4.56 \mathrm{E}-01$ & \\
\hline $\mathrm{Zr}+4$ & & $2.65 \mathrm{E}-01$ & & $1.26 \mathrm{E}+00$ & $0.00 \mathrm{E}+00$ & $3.84 \mathrm{E}-01$ & $7.95 \mathrm{E}-02$ & \\
\hline $\mathrm{Al}(\mathrm{OH}) 4-$ & & $3.34 \mathrm{E}+03$ & & $7.08 \mathrm{E}+03$ & $0.00 \mathrm{E}+\infty$ & $2.16 \mathrm{E}+03$ & $4.48 \mathrm{E}+02$ & \\
\hline $\mathrm{C}_{2} \mathrm{O}-2$ & & $2.48 \mathrm{E}+03$ & & $1.68 \mathrm{E}+04$ & $0.00 \mathrm{E}+00$ & $6.09 \mathrm{E}+03$ & $1.26 \mathrm{E}+03$ & \\
\hline $\mathrm{Cl}-$ & & $1.82 \mathrm{E}+01$ & & $2.43 \mathrm{E}+01$ & $0.00 \mathrm{E}+00$ & $7.42 \mathrm{E}+00$ & $1.54 \mathrm{E}+00$ & \\
\hline $\mathrm{CO} 3-2$ & & $1.78 \mathrm{E}+03$ & & $6.17 \mathrm{E}+04$ & $0.00 \mathrm{E}+00$ & $1.88 \mathrm{E}+04$ & $3.90 \mathrm{E}+03$ & \\
\hline$\overline{\mathrm{Cr}(\mathrm{OH}) 4-}$ & & $1.48 \mathrm{E}+\infty 0$ & & $8.67 \mathrm{E}+00$ & $0.00 \mathrm{E}+00$ & $2.65 \mathrm{E}+00$ & $5.48 \mathrm{E}-01$ & \\
\hline $\mathrm{CrO} 4-2$ & & $1.45 \mathrm{E}+02$ & & $4.22 \mathrm{E}+03$ & $0.00 \mathrm{E}+00$ & $1.29 \mathrm{E}+03$ & $2.67 \mathrm{E}+02$ & \\
\hline F- & & $1.58 \mathrm{E}+02$ & & $2.05 \mathrm{E}+03$ & $0.00 \mathrm{E}+00$ & $6.25 \mathrm{E}+02$ & $1.29 \mathrm{E}+02$ & \\
\hline HCO3. & & & & & $0.00 \mathrm{E}+00$ & & & \\
\hline NO2- & & $6.18 \mathrm{E}+03$ & & $8.01 \mathrm{E}+04$ & $3.70 \mathrm{E}+02$ & $2.48 \mathrm{E}+04$ & $5.14 \mathrm{E}+03$ & $3.70 \mathrm{E}+02$ \\
\hline NO3- & & $6.87 \mathrm{E}+03$ & & $6.12 \mathrm{E}+04$ & $0.00 \mathrm{E}+00$ & $1.87 \mathrm{E}+04$ & $3.87 \mathrm{E}+03$ & \\
\hline OH. & & $2.04 \mathrm{E}+03$ & & $4.69 \mathrm{E}+03$ & $1.37 \mathrm{E}+03$ & $2.80 \mathrm{E}+03$ & $5.79 \mathrm{E}+02$ & $1.37 \mathrm{E}+03$ \\
\hline PO4-3 & & $1.17 \mathrm{E}+02$ & & $4.63 \mathrm{E}+02$ & $0.00 \mathrm{E}+00$ & $1.41 \mathrm{E}+02$ & $2.92 \mathrm{E}+01$ & \\
\hline SO4-2 & & $1.64 \mathrm{E}+03$ & & $4.76 \mathrm{E}+04$ & $0.00 \mathrm{E}+00$ & $1.45 \mathrm{E}+04$ & $3.01 \mathrm{E}+03$ & \\
\hline $\mathrm{H} 2 \mathrm{O}$ & $5.46 \mathrm{E}+05$ & $1.16 \mathrm{E}+06$ & $1.68 \mathrm{E}+06$ & $1.18 \mathrm{E}+06$ & $8.03 \mathrm{E}+05$ & $1.16 \mathrm{E}+06$ & $2.41 E+05$ & $8.03 E+05$ \\
\hline
\end{tabular}


HNF-SD-TWR-AGA-003

Revision 0

Table C-2. Alternative 2 Material Balance.

(Sheet 6 of 12)

\begin{tabular}{|l|c|c|c|c|c|c|c|c|}
\hline STREAM NAME & 7 & 8 & $2 \mathrm{~B}$ & 9 & $10 \mathrm{~A}$ & $11 \mathrm{~A}$ & $12 \mathrm{~A}$ & $10 \mathrm{~B}$ \\
\hline SOLID COMPONENTS & & & & & & & & \\
\hline Total Mass Flow $(\mathrm{kg})$ & & $1.30 \mathrm{E}+05$ & & $7.51 \mathrm{E}+02$ & $0.00 \mathrm{E}+00$ & $2.25 \mathrm{E}+05$ & $7.43 \mathrm{E}+02$ & \\
\hline
\end{tabular}

Radionuclides (Ci)

\begin{tabular}{|l|l|l|l|l|l|l|l|l|}
\hline $\mathrm{Pu}-239$ & & $9.46 \mathrm{E}+02$ & & $4.14 \mathrm{E}+00$ & $0.00 \mathrm{E}+00$ & $1.25 \mathrm{E}+03$ & $4.12 \mathrm{E}+00$ & \\
\hline $\mathrm{Sr}-90$ & & $6.25 \mathrm{E}+06$ & & $1.22 \mathrm{E}+04$ & $0.00 \mathrm{E}+00$ & $3.68 \mathrm{E}+06$ & $1.21 \mathrm{E}+04$ & \\
\hline $\mathrm{Y}-90$ & & $6.25 \mathrm{E}+06$ & & $1.22 \mathrm{E}+04$ & $0.00 \mathrm{E}+00$ & $3.68 \mathrm{E}+06$ & $1.21 \mathrm{E}+04$ & \\
\hline Total Curies & & $1.25 \mathrm{E}+07$ & & $2.44 \mathrm{E}+04$ & $0.00 \mathrm{E}+00$ & $7.36 \mathrm{E}+06$ & $2.43 \mathrm{E}+04$ & \\
\hline
\end{tabular}

Chemicals (kg)

\begin{tabular}{|c|c|c|c|c|c|c|c|}
\hline $\mathrm{Ag}+$ & & & & $0.00 \mathrm{E}+00$ & & & \\
\hline $\mathrm{Al}+3$ & $1.44 \mathrm{E}+04$ & & $8.27 \mathrm{E}+01$ & $0.00 \mathrm{E}+00$ & $2.50 \mathrm{E}+04$ & $8.24 \mathrm{E}+01$ & \\
\hline$\overline{B i+3}$ & $3.45 \mathrm{E}+01$ & & $1.10 \mathrm{E}-01$ & $0.00 \mathrm{E}+00$ & $3.32 \mathrm{E}+01$ & $1.09 \mathrm{E}-01$ & \\
\hline $\mathrm{Ca}+2$ & $5.65 \mathrm{E}+02$ & & $3.65 \mathrm{E}+00$ & $0.00 \mathrm{E}+\infty$ & $1.10 \mathrm{E}+03$ & $3.64 \mathrm{E}+00$ & \\
\hline $\mathrm{Cr}+3$ & $3.26 \mathrm{E}+02$ & & $2.07 \mathrm{E}+00$ & $0.00 \mathrm{E}+00$ & $6.26 \mathrm{E}+02$ & $2.07 \mathrm{E}+00$ & \\
\hline $\mathrm{Fe}+3$ & $2.32 \mathrm{E}+04$ & & $1.61 \mathrm{E}+02$ & $0.00 \mathrm{E}+00$ & $4.86 \mathrm{E}+04$ & $1.60 \mathrm{E}+02$ & \\
\hline $\mathrm{La}+3$ & $8.87 \mathrm{E}+02$ & & $5.53 \mathrm{E}+00$ & $0.00 \mathrm{E}+00$ & $1.67 \mathrm{E}+03$ & $5.51 \mathrm{E}+00$ & \\
\hline $\mathrm{Mg}+2$ & & $i$ & & $0.00 \mathrm{E}+00$ & & & \\
\hline $\mathrm{Mn}+4$ & $5.17 \mathrm{E}+03$ & & $3.51 \mathrm{E}+00$ & $0.00 \mathrm{E}+00$ & $1.06 \mathrm{E}+03$ & $3.50 \mathrm{E}+00$ & \\
\hline $\mathrm{Nat}$ & $1.90 \mathrm{E}+02$ & & $4.70 \mathrm{E}+00$ & $0.00 \mathrm{E}+00$ & $9.22 \mathrm{E}+02$ & $3.04 \mathrm{E}+00$ & \\
\hline $\mathrm{Ni}+3$ & $1.34 \mathrm{E}+03$ & & $1.09 \mathrm{E}+01$ & $0.00 \mathrm{E}+00$ & $3.28 \mathrm{E}+03$ & $1.08 \mathrm{E}+01$ & \\
\hline $\mathrm{Pb}+4$ & $4.51 \mathrm{E}+02$ & & $1.33 \mathrm{E}+00$ & $0.00 \mathrm{E}+\infty 0$ & $4.01 \mathrm{E}+02$ & $1.32 \mathrm{E}+00$ & \\
\hline$P u+4$ & $1.53 \mathrm{E}+01$ & & $6.67 \mathrm{E}-02$ & $0.00 \mathrm{E}+00$ & $2.02 \bar{E}+01$ & $6.65 \mathrm{E}-02$ & \\
\hline $\mathrm{Sr}+2$ & $1.15 \mathrm{E}+02$ & & $4.00 \mathrm{E}-01$ & $0.00 \mathrm{E}+00$ & $1.21 \mathrm{E}+02$ & $3.99 \mathrm{E}-01$ & \\
\hline $\mathrm{UO} 2+2$ & $2.75 \mathrm{E}+03$ & & $3.59 \mathrm{E}+01$ & $0.00 \mathrm{E}+00$ & $1.09 \mathrm{E}+04$ & $3.58 \mathrm{E}+01$ & \\
\hline $\mathrm{Zr}+4$ & $8.14 \mathrm{E}+03$ & & $2.23 \mathrm{E}+01$ & $0.00 \mathrm{E}+00$ & $6.73 \overline{\mathrm{E}}+03$ & $2.22 \mathrm{E}+01$ & \\
\hline C2O4-2 & & & $3.17 \mathrm{E}+00$ & $0.00 \mathrm{E}+00$ & & & \\
\hline $\mathrm{Cl}-$ & & & $1.06 \mathrm{E}-03$ & $0.00 \mathrm{E}+00$ & $3.19 \mathrm{E}-01$ & $1.05 \mathrm{E}-03$ & \\
\hline $\mathrm{CO}-2$ & $7.84 \mathrm{E}+01$ & & $2.77 \mathrm{E}-01$ & $0.00 \mathrm{E}+00$ & $8.35 \mathrm{E}+01$ & $2.76 \mathrm{E}-01$ & \\
\hline F- & $5.36 \mathrm{E}+02$ & & $3.45 \mathrm{E}+00$ & $0.00 \mathrm{E}+00$ & $1.04 \mathrm{E}+03$ & $3.44 \mathrm{E}+00$ & \\
\hline $\mathrm{OH}-$ & $6.31 \mathrm{E}+04$ & & $3 . \overline{42 E}+02$ & $0.00 \mathrm{E}+00$ & $1.03 \mathrm{E}+05$ & $3.41 \mathrm{E}+02$ & \\
\hline$\overline{\mathrm{PO}} 4-3$ & $6.04 \mathrm{E}+02$ & & $3.79 \mathrm{E}+00$ & $0.00 \mathrm{E}+00$ & $1.15 \mathrm{E}+03$ & $3.78 \mathrm{E}+\infty 0$ & \\
\hline KAlSiO4 & $7.69 \mathrm{E}+03$ & & $6.39 \mathrm{E}+01$ & $0.00 \mathrm{E}+00$ & $1.93 \mathrm{E}+04$ & $6.37 \mathrm{E}+01$ & \\
\hline $\mathrm{NaAISiO4}$ & & & & $0.00 \mathrm{E}+00$ & & & \\
\hline
\end{tabular}




\section{HNF-SD-TWR-AGA-003}

\section{Revision 0}

Table C-2. Alternative 2 Material Balance.

(Sheet 7 of 12)

\begin{tabular}{|l|c|c|c|c|c|c|c|c|}
\hline $\begin{array}{l}\text { STREAM NAME } \\
\text { LIQUTD COMPONENTS }\end{array}$ & $11 \mathrm{~B}$ & $12 \mathrm{~B}$ & $10 \mathrm{C}$ & $11 \mathrm{C}$ & $12 \mathrm{C}$ & $10 \mathrm{D}$ & $11 \mathrm{D}$ & $12 \mathrm{D}$ \\
\hline Total Mass Flow $(\mathrm{kg})$ & $1.84 \mathrm{E}+06$ & $8.15 \mathrm{E}+05$ & $8.07 \mathrm{E}+05$ & $1.83 \mathrm{E}+06$ & $8.13 \mathrm{E}+05$ & $8.07 \mathrm{E}+05$ & $1.83 \mathrm{E}+06$ & $8.11 \mathrm{E}+05$ \\
\hline Volume (L) & $1.76 \mathrm{E}+06$ & $7.80 \mathrm{E}+05$ & $8.05 \mathrm{E}+05$ & $1.79 \mathrm{E}+06$ & $7.92 \mathrm{E}+05$ & $8.05 \mathrm{E}+05$ & $1.80 \mathrm{E}+06$ & $7.99 \mathrm{E}+05$ \\
\hline Volume (gal) & $4.65 \mathrm{E}+05$ & $2.06 \mathrm{E}+05$ & $2.13 \mathrm{E}+05$ & $4.72 \mathrm{E}+05$ & $2.09 \mathrm{E}+05$ & $2.13 \mathrm{E}+05$ & $4.75 \mathrm{E}+05$ & $2.11 \mathrm{E}+05$ \\
\hline Specific Gravity & $1.05 \mathrm{E}+00$ & $1.05 \mathrm{E}+00$ & $1.00 \mathrm{E}+00$ & $1.03 \mathrm{E}+00$ & $1.03 \mathrm{E}+00$ & $1.00 \mathrm{E}+00$ & $1.02 \mathrm{E}+00$ & $1.02 \mathrm{E}+00$ \\
\hline
\end{tabular}

Radionuclides (Ci)

\begin{tabular}{|l|l|l|l|l|l|l|l|l|}
\hline Cs-137 & $8.02 \mathrm{E}+05$ & $3.55 \mathrm{E}+05$ & & $4.47 \mathrm{E}+05$ & $1.98 \mathrm{E}+05$ & & $2.49 \mathrm{E}+05$ & $1.11 \mathrm{E}+05$ \\
\hline Ba-137 & $7.62 \mathrm{E}+05$ & $3.37 \mathrm{E}+05$ & & $4.25 \mathrm{E}+05$ & $1.88 \mathrm{E}+05$ & & $2.37 \mathrm{E}+05$ & $1.05 \mathrm{E}+05$ \\
\hline St-90 & $9.81 \mathrm{E}+02$ & $4.34 \mathrm{E}+02$ & & $5.47 \mathrm{E}+02$ & $2.42 \mathrm{E}+02$ & & $3.04 \mathrm{E}+02$ & $1.35 \mathrm{E}+02$ \\
\hline Y-90 & $9.81 \mathrm{E}+02$ & $4.34 \mathrm{E}+02$ & & $5.47 \mathrm{E}+02$ & $2.42 \mathrm{E}+02$ & & $3.04 \mathrm{E}+02$ & $1.35 \mathrm{E}+02$ \\
\hline Total Curies & $1.57 \mathrm{E}+06$ & $6.93 \mathrm{E}+05$ & & $8.73 \mathrm{E}+05$ & $3.87 \mathrm{E}+05$ & & $4.86 \mathrm{E}+05$ & $2.16 \mathrm{E}+05$ \\
\hline
\end{tabular}

Chemicals ( $\mathrm{kg}$ )

\begin{tabular}{|c|c|c|c|c|c|c|c|c|}
\hline$\longdiv { B \dot { i } + 3 }$ & $3.25 \mathrm{E}-01$ & $1.44 \mathrm{E}-01$ & & $1.81 \mathrm{E}-01$ & $8.04 E-02$ & & $1.01 \mathrm{E}-01$ & $4.48 \mathrm{E}-02$ \\
\hline $\mathrm{Ca}+2$ & $1.12 \mathrm{E}-01$ & $4.94 \mathrm{E}-02$ & & $6.22 \mathrm{E}-02$ & $2.76 \mathrm{E}-02$ & & $3.46 \mathrm{E}-02$ & $1.54 \mathrm{E}-02$ \\
\hline $\mathrm{Cs}+$ & $9.26 \mathrm{E}+00$ & $4.10 \mathrm{E}+00$ & & $5.16 \mathrm{E}+00$ & $2.29 \mathrm{E}+00$ & & $2.87 \mathrm{E}+00$ & $1.28 \mathrm{E}+00$ \\
\hline \multicolumn{9}{|l|}{$\mathrm{Fe}+3$} \\
\hline $\mathrm{K}+$ & $1.18 \mathrm{E}+03$ & $5.23 \mathrm{E}+02$ & & $6.58 \mathrm{E}+02$ & $2.92 \mathrm{E}+02$ & & $3.67 \mathrm{E}+02$ & $1.63 \mathrm{E}+02$ \\
\hline $\mathrm{Mg}+2$ & & & & & & & $0.00 \mathrm{E}+\infty 0$ & \\
\hline $\mathrm{Mn}+4$ & $1.02 \mathrm{E}-01$ & $4.51 \mathrm{E}-02$ & & $5.68 \mathrm{E}-02$ & 2.52E-02 & & $3.16 \mathrm{E}-02$ & $1.40 \mathrm{E}-02$ \\
\hline $\mathrm{Na}+$ & $4.06 \mathrm{E}+04$ & $1.80 \mathrm{E}+04$ & $2.04 \mathrm{E}+03$ & $2 . \overline{47 E}+04$ & $1.09 \mathrm{E}+04$ & $2.04 \mathrm{E}+03$ & $1.58 \mathrm{E}+04$ & $7.00 \mathrm{E}+03$ \\
\hline \multicolumn{9}{|l|}{$\mathrm{Ni}+3$} \\
\hline$S i+4$ & $9.79 \mathrm{E}-01$ & $4.33 \mathrm{E}-01$ & & $5.46 \mathrm{E}-01$ & $2.42 \mathrm{E}-01$ & & $3.04 \mathrm{E}-01$ & $1.35 \mathrm{E}-01$ \\
\hline $\mathrm{Sr}+2$ & 3.21E-02 & $1.42 \mathrm{E}-02$ & & $1.79 \mathrm{E}-02$ & $7.94 \mathrm{E}-03$ & & $9.97 \mathrm{E}-03$ & $4.43 \mathrm{E}-03$ \\
\hline $\mathrm{UO2+2}$ & $1.75 \mathrm{E}+00$ & $7.73 \mathrm{E}-01$ & & $9.74 \mathrm{E}-01$ & $4.32 \mathrm{E}-01$ & & $5.42 \mathrm{E}-01$ & $2.41 \mathrm{E}-01$ \\
\hline $\mathrm{Zr}+4$ & 3.05E-01 & $1.35 \mathrm{E}-01$ & & $1.70 \mathrm{E}-01$ & $7.52 \mathrm{E}-02$ & & $9.45 \mathrm{E}-02$ & $4.20 \mathrm{E}-02$ \\
\hline $\mathrm{Al}(\mathrm{OH}) 4-$ & $1.72 \mathrm{E}+03$ & $7.59 \mathrm{E}+02$ & & $9.56 \mathrm{E}+02$ & $4.24 E+02$ & & $5.32 \mathrm{E}+02$ & $2.36 \mathrm{E}+02$ \\
\hline C2O4-2 & $4.83 \mathrm{E}+03$ & $2.14 \mathrm{E}+03$ & & $2.69 \mathrm{E}+03$ & $1.19 \mathrm{E}+03$ & & $1.50 \mathrm{E}+03$ & $6.65 \mathrm{E}+02$ \\
\hline Cl- & $5.88 E+\infty$ & $2.60 \mathrm{E}+00$ & & $3.28 \mathrm{E}+00$ & $1.45 \mathrm{E}+\infty 0$ & & $1.83 \mathrm{E}+00$ & $8.10 \mathrm{E}-01$ \\
\hline $\mathrm{CO} 3-2$ & $1.49 \mathrm{E}+04$ & $6.61 \mathrm{E}+03$ & & $8.33 \mathrm{E}+03$ & $3.69 \mathrm{E}+03$ & & $4.64 \mathrm{E}+03$ & $2.06 \mathrm{E}+03$ \\
\hline $\mathrm{Cr}(\mathrm{OH}) 4-$ & $2.10 \mathrm{E}+00$ & $9.29 \mathrm{E}-01$ & & $1.17 \mathrm{E}+00$ & $5.19 \mathrm{E}-01$ & & $6.51 \mathrm{E}-01$ & $2.89 \mathrm{E}-01$ \\
\hline $\mathrm{CrO4-2}$ & $1.02 \mathrm{E}+03$ & $4.52 E+02$ & & $5.69 \mathrm{E}+02$ & $2.52 \mathrm{E}+02$ & & $3.17 \mathrm{E}+02$ & $1.41 E+02$ \\
\hline F- & $4.95 \mathrm{E}+02$ & $2.19 \mathrm{E}+02$ & & $2.76 \mathrm{E}+02$ & $1.22 \mathrm{E}+02$ & & $1.54 \mathrm{E}+02$ & $6.82 \mathrm{E}+01$ \\
\hline HCO3- & & & & & & & $0.00 \mathrm{E}+00$ & \\
\hline NO2- & $2.01 E+04$ & $8.88 \mathrm{E}+03$ & $3.70 \mathrm{E}+02$ & $1.16 \mathrm{E}+04$ & $5.12 \mathrm{E}+03$ & $3.70 \mathrm{E}+02$ & $6.80 \mathrm{E}+03$ & $3.02 \mathrm{E}+03$ \\
\hline NO3- & $1.48 \mathrm{E}+04$ & $6.56 \mathrm{E}+03$ & & $8.25 \mathrm{E}+03$ & $3.66 \mathrm{E}+03$ & & $4.60 \mathrm{E}+03$ & $2.04 \mathrm{E}+03$ \\
\hline OH- & $3.59 \mathrm{E}+03$ & $1.59 \mathrm{E}+03$ & $1.37 \mathrm{E}+03$ & $3.37 \mathrm{E}+03$ & $1.49 \mathrm{E}+03$ & $1.37 \mathrm{E}+03$ & $3.24 \mathrm{E}+03$ & $1.44 \mathrm{E}+03$ \\
\hline PO4-3 & $1.12 \mathrm{E}+02$ & $4.96 \mathrm{E}+01$ & & $6.24 \mathrm{E}+01$ & $2.77 \mathrm{E}+01$ & & $3.48 \mathrm{E}+01$ & $1.54 \mathrm{E}+01$ \\
\hline SO4-2 & $1.15 \mathrm{E}+04$ & $5.10 \mathrm{E}+03$ & & $6.42 \mathrm{E}+03$ & $2.85 \mathrm{E}+03$ & & $3.58 \mathrm{E}+03$ & $1.59 \mathrm{E}+03$ \\
\hline $\mathrm{H} 2 \mathrm{O}$ & $1.73 \mathrm{E}+06$ & $7.64 \mathrm{E}+05$ & $8.03 \mathrm{E}+05$ & $1.77 \mathrm{E}+06$ & $7.82 \mathrm{E}+05$ & $8.03 E+05$ & $1.79 \mathrm{E}+06$ & $7.93 \mathrm{E}+05$ \\
\hline
\end{tabular}




\section{HNF-SD-TWR-AGA-003}

\section{Revision 0}

Table C-2. Alternative 2 Material Balance.

(Sheet 8 of 12)

\begin{tabular}{|l|c|c|c|c|c|c|c|c|}
\hline STREAM NAME & $11 \mathrm{~B}$ & $12 \mathrm{~B}$ & $10 \mathrm{C}$ & $1 \mathrm{C}$ & $12 \mathrm{C}$ & $10 \mathrm{D}$ & $11 \mathrm{D}$ & $12 \mathrm{D}$ \\
\hline SOLID COMPONENTS & & & & & & & & \\
\hline Total Mass Flow (kg) & $2.25 \mathrm{E}+05$ & $7.41 \mathrm{E}+02$ & & $2.24 \mathrm{E}+05$ & $7.39 \mathrm{E}+02$ & & $2.23 \mathrm{E}+05$ & $7.36 \mathrm{E}+02$ \\
\hline
\end{tabular}

Radionuclides (Ci)

\begin{tabular}{|l|l|l|l|l|l|l|l|l|}
\hline Pu-239 & $1.25 \mathrm{E}+03$ & $4.11 \mathrm{E}+00$ & & $1.24 \mathrm{E}+03$ & $4.10 \mathrm{E}+00$ & & $1.24 \mathrm{E}+03$ & $4.08 \mathrm{E}+00$ \\
\hline ST-90 & $3.67 \mathrm{E}+06$ & $1.21 \mathrm{E}+04$ & & $3.66 \mathrm{E}+06$ & $1.21 \mathrm{E}+04$ & & $3.64 \mathrm{E}+06$ & $1.20 \mathrm{E}+04$ \\
\hline Y-90 & $3.67 \mathrm{E}+06$ & $1.21 \mathrm{E}+04$ & & $3.66 \mathrm{E}+06$ & $1.21 \mathrm{E}+04$ & & $3.64 \mathrm{E}+06$ & $1.20 \mathrm{E}+04$ \\
\hline Total Curies & $7.34 \mathrm{E}+06$ & $2.42 \mathrm{E}+04$ & & $7.31 \mathrm{E}+06$ & $2.41 \mathrm{E}+04$ & & $7.29 \mathrm{E}+06$ & $2.41 \mathrm{E}+04$ \\
\hline
\end{tabular}

Chemicals (kg)

\begin{tabular}{|c|c|c|c|c|c|c|c|}
\hline $\mathrm{Ag}+$ & & & & & & $0.00 \mathrm{E}+00$ & \\
\hline $\mathrm{Al}+3$ & $2.49 \mathrm{E}+04$ & $8.21 E+01$ & & $2.48 \mathrm{E}+04$ & $8.19 \mathrm{E}+01$ & $2.47 \mathrm{E}+04$ & $8.16 \mathrm{E}+01$ \\
\hline$\overline{\mathrm{Bi}+3}$ & $3.30 \mathrm{E}+01$ & $1.09 \mathrm{E}-0 \mathrm{I}$ & & $3.29 \mathrm{E}+01$ & $1.09 \mathrm{E}-01$ & $3.28 \mathrm{E}+01$ & $1.08 \mathrm{E}-01$ \\
\hline $\mathrm{Ca}+2$ & $1.10 \mathrm{E}+03$ & $3.63 E+00$ & & $1.10 \mathrm{E}+03$ & $3.61 \mathrm{E}+00$ & $1.09 \mathrm{E}+03$ & $3.60 \mathrm{E}+00$ \\
\hline $\mathrm{Cr}+3$ & $6.24 \mathrm{E}+02$ & $2.06 \mathrm{E}+00$ & & $6.22 \mathrm{E}+02$ & $2.05 \mathrm{E}+\infty$ & $6.20 \mathrm{E}+02$ & $2.05 \mathrm{E}+00$ \\
\hline $\mathrm{Fe}+3$ & $4.84 \mathrm{E}+04$ & $1.60 \mathrm{E}+02$ & & $4.83 \mathrm{E}+04$ & $1.59 \mathrm{E}+02$ & $4.81 \mathrm{E}+04$ & $1.59 \mathrm{E}+02$ \\
\hline $\mathrm{La}+3$ & $1.67 \mathrm{E}+03$ & $5.50 \mathrm{E}+00$ & & $1.66 \mathrm{E}+03$ & $5.48 \mathrm{E}+00$ & $1.65 \mathrm{E}+03$ & $5.46 \mathrm{E}+00$ \\
\hline $\mathrm{Mg}+2$ & & & $\because$ & & & $0.00 \mathrm{E}+00$ & \\
\hline $\mathrm{Mn}+4$ & $1.06 \mathrm{E}+03$ & $3.48 \mathrm{E}+00$ & & $1.05 \mathrm{E}+03$ & $3.47 \mathrm{E}+00$ & $1.05 \mathrm{E}+03$ & $3.46 \mathrm{E}+00$ \\
\hline $\mathrm{Na}+$ & $9.19 \mathrm{E}+02$ & $3.03 \mathrm{E}+00$ & & $9.16 E+02$ & $3.02 \mathrm{E}+\infty 0$ & $9.13 \mathrm{E}+02$ & $3.01 \mathrm{E}+00$ \\
\hline $\mathrm{Ni}+3$ & $3.27 \mathrm{E}+03$ & $1.08 \mathrm{E}+01$ & & $3.26 E+03$ & $1.08 \mathrm{E}+01$ & $3.25 \mathrm{E}+03$ & $1.07 \mathrm{E}+01$ \\
\hline $\mathrm{Pb}+4$ & $4.00 \mathrm{E}+02$ & $1.32 \mathrm{E}+00$ & & $3.99 \mathrm{E}+02$ & $1.32 \mathrm{E}+\infty$ & $3.97 \mathrm{E}+02$ & $1.31 \mathrm{E}+\infty$ \\
\hline $\mathrm{Pu}+4$ & $2.01 \mathrm{E}+01$ & $6.63 \mathrm{E}-02$ & & $2.00 \mathrm{E}+01$ & $6.61 \mathrm{E}-02$ & $2.00 \mathrm{E}+01$ & $6.58 \mathrm{E}-02$ \\
\hline $\mathrm{Sr}+2$ & $1.20 \mathrm{E}+02$ & $3.97 \mathrm{E}-01$ & & $1.20 \mathrm{E}+02$ & $3.96 \mathrm{E}-01$ & $1.20 \mathrm{E}+02$ & $3.95 \mathrm{E}-01$ \\
\hline $\mathrm{UO} 2+2$ & $1.08 \mathrm{E}+04$ & $3.57 \mathrm{E}+01$ & & $1.08 \mathrm{E}+04$ & $3.56 \mathrm{E}+01$ & $1.07 \mathrm{E}+04$ & $3.55 \mathrm{E}+01$ \\
\hline$\overline{Z r+4}$ & $6.71 \mathrm{E}+03$ & $2.22 \mathrm{E}+0 \mathrm{I}$ & & $6.69 \mathrm{E}+03$ & $2.21 \mathrm{E}+01$ & $6.67 \mathrm{E}+03$ & $2.20 \mathrm{E}+01$ \\
\hline $\mathrm{C} 2 \mathrm{O} 4-2$ & & & & & & $0.00 \mathrm{E}+00$ & \\
\hline $\mathrm{Cl}-$ & $3.18 \mathrm{E}-01$ & $1.05 \mathrm{E}-03$ & & $3.17 \mathrm{E}-01$ & $1.05 \mathrm{E}-03$ & $3.16 \mathrm{E}-01$ & $1.04 \mathrm{E}-03$ \\
\hline CO3-2 & $8.33 \mathrm{E}+01$ & $2.75 \mathrm{E}-01$ & & $8.30 \mathrm{E}+01$ & $2.74 \mathrm{E}-01$ & $8.27 \mathrm{E}+01$ & $2.73 \mathrm{E}-01$ \\
\hline F- & $1.04 \mathrm{E}+03$ & $3.43 \mathrm{E}+00$ & & $1.04 \mathrm{E}+03$ & $3.42 \mathrm{E}+00$ & $1.03 \mathrm{E}+03$ & $3.41 \mathrm{E}+00$ \\
\hline $\mathrm{OH}-$ & $1.03 \mathrm{E}+05$ & $3.40 \mathrm{E}+02$ & & $1.03 \mathrm{E}+05$ & $3.39 \mathrm{E}+02$ & $1.02 \mathrm{E}+05$ & $3.38 \mathrm{E}+02$ \\
\hline PO4-3 & $1.14 \mathrm{E}+03$ & $3.77 \mathrm{E}+\infty$ & & $1.14 \mathrm{E}+03$ & $3.76 \mathrm{E}+00$ & $1.13 \mathrm{E}+03$ & $3.74 \mathrm{E}+00$ \\
\hline KAISiO4 & $1.92 \mathrm{E}+04$ & $6.35 \mathrm{E}+01$ & & $1.92 \mathrm{E}+04$ & $6.32 \mathrm{E}+01$ & $1.91 \mathrm{E}+04$ & $6.30 \mathrm{E}+01$ \\
\hline NaA1SiO4 & & & & & & $0.00 \mathrm{E}+00$ & \\
\hline
\end{tabular}


HNF-SD-TWR-AGA-003

Revision 0

Table C-2. Alternative 2 Material Balance.

(Sheet 9 of 12)

\begin{tabular}{|l|c|c|c|c|c|c|c|c|}
\hline $\begin{array}{l}\text { STREAM NAME } \\
\text { LIQUID COMPONENTS }\end{array}$ & 13 & 14 & 15 & $16 \mathrm{~A}$ & $17 \mathrm{~A}$ & $18 \mathrm{~A}$ & $16 \mathrm{~B}$ & $17 \mathrm{~B}$ \\
\hline Total Mass Flow (kg) & $8.17 \mathrm{E}+05$ & $1.83 \mathrm{E}+06$ & $2.18 \mathrm{E}+06$ & $1.23 \mathrm{E}+06$ & $2.79 \mathrm{E}+06$ & $1.42 \mathrm{E}+06$ & $1.23 \mathrm{E}+06$ & $2.60 \mathrm{E}+06$ \\
\hline Volume (L) & $8.17 \mathrm{E}+05$ & $1.82 \mathrm{E}+06$ & $2.01 \mathrm{E}+06$ & $1.23 \mathrm{E}+06$ & $2.65 \mathrm{E}+06$ & $1.35 \mathrm{E}+06$ & $1.23 \mathrm{E}+06$ & $2.53 \mathrm{E}+06$ \\
\hline Volume (gal) & $2.16 \mathrm{E}+05$ & $4.80 \mathrm{E}+05$ & $5.31 \mathrm{E}+05$ & $3.24 \mathrm{E}+05$ & $7.01 \mathrm{E}+05$ & $3.57 \mathrm{E}+05$ & $3.24 \mathrm{E}+05$ & $6.68 \mathrm{E}+05$ \\
\hline Specific Gravity & $1.00 \mathrm{E}+00$ & $1.01 \mathrm{E}+00$ & $1.08 \mathrm{E}+00$ & $1.00 \mathrm{E}+00$ & $1.05 \mathrm{E}+00$ & $1.05 \mathrm{E}+00$ & $1.00 \mathrm{E}+00$ & $1.03 \mathrm{E}+00$ \\
\hline
\end{tabular}

Radionuclides (Ci)

\begin{tabular}{|c|c|c|c|c|c|}
\hline Cs-137 & $1.38 \mathrm{E}+05$ & $3.09 \mathrm{E}+05$ & $2.17 \mathrm{E}+05$ & $1.10 \mathrm{E}+05$ & $1.06 \mathrm{E}+05$ \\
\hline Ba-137 & $1.32 \mathrm{E}+05$ & $2.94 \mathrm{E}+05$ & $2.06 \mathrm{E}+05$ & $1.05 \mathrm{E}+05$ & $1.01 \mathrm{E}+05$ \\
\hline Sr-90 & $1.69 \mathrm{E}+02$ & & & & \\
\hline $\mathrm{Y}-90$ & $1.69 \mathrm{E}+02$ & & & & \\
\hline Total Curies & $2.70 \mathrm{E}+05$ & $6.03 \mathrm{E}+05$ & $4.22 \mathrm{E}+05$ & $2.15 \mathrm{E}+05$ & $2.07 \mathrm{E}+05$ \\
\hline
\end{tabular}

Chemicals (kg)

\begin{tabular}{|c|c|c|c|c|c|c|c|c|}
\hline $\mathrm{Bi}+3$ & & $5.61 \mathrm{E}-02$ & & & & & & \\
\hline $\mathrm{Ca}+2$ & & $1.92 \mathrm{E}-02$ & $2.12 \mathrm{E}+00$ & & $1.49 \mathrm{E}+00$ & $7.57 \mathrm{E}-01$ & & $7.30 \mathrm{E}-01$ \\
\hline Cst & & $1.60 \mathrm{E}+00$ & $3.57 \mathrm{E}+00$ & & $2.50 \mathrm{E}+00$ & $1.27 \mathrm{E}+00$ & & $1.23 \mathrm{E}+00$ \\
\hline $\mathrm{Fe}+3$ & & & i & & & & & \\
\hline $\mathrm{K}+$ & & $2.04 \mathrm{E}+02$ & $8.74 \mathrm{E}+02$ & & $6.12 \mathrm{E}+02$ & $3.12 \mathrm{E}+02$ & & $3.00 \mathrm{E}+02$ \\
\hline $\mathrm{Mg}+2$ & & & $1.17 \mathrm{E}+\infty$ & & $8.22 \mathrm{E}-01$ & $4.19 \mathrm{E}-01$ & & $4.03 E-01$ \\
\hline $\mathrm{Mn}+4$ & & $1.76 \mathrm{E}-02$ & $2.06 \mathrm{E}+01$ & & $1.45 \mathrm{E}+01$ & $7.36 \mathrm{E}+00$ & & $7.09 \mathrm{E}+00$ \\
\hline $\mathrm{Na}+$ & & $8.76 \mathrm{E}+03$ & $9.71 E+04$ & $3.10 \mathrm{E}+03$ & $8.37 \mathrm{E}+04$ & $4.26 \mathrm{E}+04$ & $3.10 \mathrm{E}+03$ & $4.42 \mathrm{E}+04$ \\
\hline \multicolumn{9}{|l|}{$\mathrm{Ni}+3$} \\
\hline $\mathrm{Si}+4$ & & $1.69 \mathrm{E}-01$ & $1.97 \mathrm{E}+01$ & & $1.38 \mathrm{E}+01$ & $7.03 E+00$ & & $6.78 \mathrm{E}+00$ \\
\hline $\mathbf{S r}+2$ & & $5.54 \mathrm{E}-03$ & & & & & & \\
\hline $\mathrm{UO} 2+2$ & & $3.01 \mathrm{E}-01$ & $2.38 \mathrm{E}+03$ & & $1.67 \mathrm{E}+03$ & $8.48 \mathrm{E}+02$ & & $8.17 \mathrm{E}+02$ \\
\hline$Z r+4$ & & $5.25 \mathrm{E}-02$ & $1.07 \mathrm{E}-01$ & & $7.51 \mathrm{E}-02$ & $3.82 \mathrm{E}-02$ & & 3.69E-02 \\
\hline $\mathrm{Al}(\mathrm{OH}) 4-$ & & $2.96 \mathrm{E}+02$ & $2.72 \mathrm{E}+03$ & & $1.90 \mathrm{E}+03$ & $9.69 \mathrm{E}+02$ & & $9.34 \mathrm{E}+02$ \\
\hline $\mathrm{C} 2 \mathrm{O} 4-2$ & & $8.33 E+02$ & $2.43 \mathrm{E}+04$ & & $4.13 \mathrm{E}+04$ & $2.10 \mathrm{E}+04$ & & $2.03 \mathrm{E}+04$ \\
\hline $\mathrm{Cl}-$ & & $1.02 \mathrm{E}+00$ & $6.9 \mathrm{IE}+02$ & & $4.84 \mathrm{E}+02$ & $2.46 \mathrm{E}+02$ & & $2.38 \mathrm{E}+02$ \\
\hline $\mathrm{CO} 3-2$ & & $2.58 \mathrm{E}+03$ & $7.06 \mathrm{E}+04$ & & $4.95 \mathrm{E}+04$ & $2.52 \mathrm{E}+04$ & & $2.43 \mathrm{E}+04$ \\
\hline $\mathrm{Cr}(\mathrm{OH}) 4-$ & & $3.62 \mathrm{E}-01$ & $2.12 \mathrm{E}-01$ & & $1.48 \mathrm{E}-01$ & $7.56 \mathrm{E}-02$ & & $7.28 \mathrm{E}-02$ \\
\hline CrO4-2 & & $1.76 \mathrm{E}+02$ & & & & & & \\
\hline F- & & $8.54 \mathrm{E}+01$ & $1.60 \mathrm{E}+02$ & & $1.12 \mathrm{E}+02$ & $5.69 \mathrm{E}+01$ & & $5.48 \mathrm{E}+01$ \\
\hline HCO3- & & & $2.39 \mathrm{E}+04$ & & $1.68 \mathrm{E}+04$ & $8.53 \mathrm{E}+03$ & & $8.23 \mathrm{E}+03$ \\
\hline NO2- & & $3.78 \mathrm{E}+03$ & $1.41 E+04$ & $5.63 \mathrm{E}+02$ & $1.05 \mathrm{E}+04$ & $5.32 \mathrm{E}+03$ & $5.63 \mathrm{E}+02$ & $5.70 \mathrm{E}+03$ \\
\hline NO3- & & $2.56 \mathrm{E}+03$ & $1.74 \mathrm{E}+03$ & & $1.22 \mathrm{E}+03$ & $6.20 \mathrm{E}+02$ & & $5.97 \mathrm{E}+02$ \\
\hline $\mathrm{OH}-$ & & $1.80 \mathrm{E}+03$ & $6.45 \mathrm{E}+03$ & $2.08 \mathrm{E}+03$ & $6.60 \mathrm{E}+03$ & $3.36 \mathrm{E}+03$ & $2.08 \mathrm{E}+03$ & $5.32 \mathrm{E}+03$ \\
\hline PO4-3 & & $1.93 E+01$ & $3.92 \mathrm{E}+03$ & & $2.74 E+03$ & $1.40 \mathrm{E}+03$ & & 1.35E+03 \\
\hline SO4-2 & & $1.99 \mathrm{E}+03$ & $3.98 \mathrm{E}+03$ & & $2.79 \mathrm{E}+03$ & $1.42 \mathrm{E}+03$ & & $1.37 \mathrm{E}+03$ \\
\hline $\mathrm{H} 2 \mathrm{O}$ & $8.17 \mathrm{E}+05$ & $1.81 E+06$ & $1.93 \mathrm{E}+06$ & $1.22 \mathrm{E}+06$ & $2.57 \mathrm{E}+06$ & $1.31 \mathrm{E}+06$ & $1.22 \mathrm{E}+06$ & $2.49 \mathrm{E}+06$ \\
\hline
\end{tabular}




\section{HNF-SD-TWR-AGA-003 \\ Revision 0}

Table C-2. Alternative 2 Material Balance.

(Sheet 10 of 12)

\begin{tabular}{|l|c|c|c|c|c|c|c|c|}
\hline STREAM NAME & 13 & 14 & 15 & $16 \mathrm{~A}$ & $17 \mathrm{~A}$ & $18 \mathrm{~A}$ & $16 \mathrm{~B}$ & $17 \mathrm{~B}$ \\
\hline SOLD COMPONENTS & & & & & & & & \\
\hline Totai Mass Flow $(\mathrm{kg})$ & & $2.22 \mathrm{E}+05$ & $1.15 \mathrm{E}+03$ & & $3.11 \mathrm{E}+05$ & $1.03 \mathrm{E}+03$ & & $3.10 \mathrm{E}+05$ \\
\hline
\end{tabular}

Radionuclides ( $\mathrm{Ci}$ )

\begin{tabular}{|l|l|l|l|l|l|l|l|l|}
\hline Pu-239 & & $1.23 \mathrm{E}+03$ & $1.16 \mathrm{E}+01$ & & $3.50 \mathrm{E}+03$ & $1.16 \mathrm{E}+01$ & & $3.49 \mathrm{E}+03$ \\
\hline ST-90 & & $3.63 \mathrm{E}+06$ & $1.40 \mathrm{E}+04$ & & $4.21 \mathrm{E}+06$ & $1.39 \mathrm{E}+04$ & & $4.20 \mathrm{E}+06$ \\
\hline Y-90 & & $3.63 \mathrm{E}+06$ & $1.40 \mathrm{E}+04$ & & $4.21 \mathrm{E}+06$ & $1.39 \mathrm{E}+04$ & & $4.20 \mathrm{E}+06$ \\
\hline Total Curies & & $7.26 \mathrm{E}+06$ & $2.79 \mathrm{E}+04$ & & $8.43 \mathrm{E}+06$ & $2.78 \mathrm{E}+04$ & & $8.40 \mathrm{E}+06$ \\
\hline
\end{tabular}

Chemicals $(\mathrm{kg})$

\begin{tabular}{|c|c|c|c|c|c|}
\hline $\mathrm{Ag}+$ & & $8.01 \mathrm{E}+00$ & $2.42 \mathrm{E}+03$ & $7.99 \mathrm{E}+00$ & $2.41 \mathrm{E}+03$ \\
\hline$\widehat{A l+3}$ & $2.47 \mathrm{E}+04$ & $6.79 \mathrm{E}+01$ & $2.05 \mathrm{E}+04$ & $6.76 \mathrm{E}+01$ & $2.04 \mathrm{E}+04$ \\
\hline $\mathrm{Bi}+3$ & $3.27 \mathrm{E}+01$ & $2.41 \mathrm{E}-02$ & $7.28 \mathrm{E}+\infty$ & $2.40 \mathrm{E}-02$ & $7.25 \mathrm{E}+00$ \\
\hline $\mathrm{Ca}+2$ & $1.09 \overline{\mathrm{E}}+03$ & $1.57 \mathrm{E}+01$ & $4.74 \mathrm{E}+03$ & $1.56 \mathrm{E}+01$ & $4.72 \mathrm{E}+03$ \\
\hline$\overline{C t+3}$ & $6.18 \mathrm{E}+02$ & $3.39 \mathrm{E}+00$ & $1.03 \mathrm{E}+03$ & $3.38 \mathrm{E}+00$ & $1.02 \mathrm{E}+03$ \\
\hline$\overline{\mathrm{Fe}}+3$ & $4.79 \mathrm{E}+04$ & $1.94 \mathrm{E}+02$ & $5.86 \mathrm{E}+04$ & $1.94 \mathrm{E}+02$ & $5.85 \mathrm{E}+04$ \\
\hline $\mathrm{La}+3$ & $1.65 \mathrm{E}+03$ & $1.87 \mathrm{E}+00$ & $5.66 \mathrm{E}+02$ & $1.87 \mathrm{E}+00$ & $5.64 \mathrm{E}+02$ \\
\hline $\overrightarrow{\mathrm{Mg}}+2$ & & $3.22 \mathrm{E}+00$ & $9.73 E+02$ & $3.21 E+\infty$ & $9.70 \mathrm{E}+02$ \\
\hline $\mathrm{Mn}+4$ & $1.05 \mathrm{E}+03$ & $9.35 \mathrm{E}+\infty 0$ & $2.83 \mathrm{E}+03$ & $9.32 \mathrm{E}+00$ & $2.82 \mathrm{E}+03$ \\
\hline $\mathrm{Na}+$ & $9.10 \mathrm{E}+02$ & $4.19 \mathrm{E}+01$ & & & \\
\hline $\mathrm{Ni}+3$ & $3.24 \mathrm{E}+03$ & $2.94 \mathrm{E}+00$ & $8.87 \mathrm{E}+02$ & $2.93 \mathrm{E}+00$ & $8.84 \mathrm{E}+02$ \\
\hline$\overline{\mathrm{Pb}+4}$ & $3.96 \mathrm{E}+02$ & $1.44 \mathrm{E}+01$ & $4.35 \mathrm{E}+03$ & $1.44 \mathrm{E}+01$ & $4.33 \mathrm{E}+03$ \\
\hline$P u+4$ & $1.99 \mathrm{E}+01$ & $1.87 \mathrm{E}-01$ & $5.65 \mathrm{E}+01$ & $1.86 \mathrm{E}-01$ & $5.63 \mathrm{E}+01$ \\
\hline $\mathrm{Sr}+2$ & $1.19 \mathrm{E}+02$ & $5.01 \mathrm{E}-01$ & $1.51 E+02$ & $4.99 \mathrm{E}-01$ & $1.51 \mathrm{E}+02$ \\
\hline $\mathrm{UO} 2+2$ & $1.07 \mathrm{E}+04$ & & & & \\
\hline$\overline{\mathrm{Zr}+4}$ & $6.65 \mathrm{E}+03$ & $1.94 \mathrm{E}+\infty 0$ & $5.85 \mathrm{E}+02$ & $1.93 \mathrm{E}+00$ & $5.83 \mathrm{E}+02$ \\
\hline $\mathrm{C}_{204-2}$ & & $8.04 \mathrm{E}+01$ & & & \\
\hline Cl- & $3.14 \mathrm{E}-01$ & & & & \\
\hline $\mathrm{CO}-2$ & $8.24 \mathrm{E}+01$ & $2.54 \mathrm{E}+00$ & $7.67 \mathrm{E}+02$ & $2.53 \mathrm{E}+00$ & $7.65 \mathrm{E}+02$ \\
\hline F. & $1.03 \mathrm{E}+03$ & & & & \\
\hline $\mathrm{OH}-$ & $1.02 E+05$ & $3.25 \mathrm{E}+02$ & $9.82 \mathrm{E}+04$ & $3.24 \mathrm{E}+02$ & $9.79 \mathrm{E}+04$ \\
\hline PO4-3 & $1.13 \mathrm{E}+03$ & $3.96 \mathrm{E}+01$ & $1.20 \mathrm{E}+04$ & $3.95 \mathrm{E}+01$ & $1.19 \mathrm{E}+04$ \\
\hline KAlSiO4 & $1.90 \mathrm{E}+04$ & $1.37 \mathrm{E}+00$ & $4.13 \mathrm{E}+02$ & $1.36 \mathrm{E}+00$ & $4.12 \mathrm{E}+02$ \\
\hline NaAlSiO4 & & $3.36 \mathrm{E}+02$ & $1.02 \mathrm{E}+05$ & $3.35 \mathrm{E}+02$ & $1.01 \mathrm{E}+05$ \\
\hline
\end{tabular}


HNF-SD-TWR-AGA-003

Revision 0

Table C-2. Alternative 2 Material Balance.

\begin{tabular}{|l|c|c|c|}
\hline $\begin{array}{l}\text { STREAM NAME } \\
\text { LIQUID COMPONENTS }\end{array}$ & $18 \mathrm{~B}$ & 19 & 20 \\
\hline Total Mass Flow (kg) & $1.24 \mathrm{E}+06$ & $1.57 \mathrm{E}+06$ & $2.94 \mathrm{E}+06$ \\
\hline Volume (L) & $1.20 \mathrm{E}+06$ & $1.57 \mathrm{E}+06$ & $2.90 \mathrm{E}+06$ \\
\hline Volume (gal) & $3.17 \mathrm{E}+05$ & $4.16 \mathrm{E}+05$ & $7.66 \mathrm{E}+05$ \\
\hline Specific Gravity & $1.03 \mathrm{E}+00$ & $1.00 \mathrm{E}+00$ & $1.01 \mathrm{E}+00$ \\
\hline
\end{tabular}

(Sheet 11 of 12)

Radionuclides $(\mathrm{Ci})$

\begin{tabular}{|l|l|l|l|}
\hline Cs-137 & $5.06 \mathrm{E}+04$ & & $5.58 \mathrm{E}+04$ \\
\hline Ba-137 & $4.80 \mathrm{E}+04$ & & $5.30 \mathrm{E}+04$ \\
\hline Sr-90 & $0.00 \mathrm{E}+00$ & & \\
\hline Y-90 & $0.00 \mathrm{E}+00$ & & \\
\hline Total Curies & $9.86 \mathrm{E}+04$ & & $1.09 \mathrm{E}+05$ \\
\hline
\end{tabular}

Chemicals (kg)

\begin{tabular}{|l|c|c|c|}
\hline $\mathrm{Bi}+3$ & $0.00 \mathrm{E}+00$ & & \\
\hline $\mathrm{Ca}+2$ & $3.47 \mathrm{E}-01$ & & $3.83 \mathrm{E}-01$ \\
\hline $\mathrm{Cs}+$ & $5.84 \mathrm{E}-01$ & & $6.44 \mathrm{E}-01$ \\
\hline $\mathrm{Fe}+3$ & & & $\vdots$ \\
\hline $\mathrm{K}+$ & $1.43 \mathrm{E}+02$ & & $1.58 \mathrm{E}+02$ \\
\hline $\mathrm{Mg}+2$ & $1.92 \mathrm{E}-01$ & & $2.12 \mathrm{E}-01$ \\
\hline $\mathrm{Mn}+4$ & $3.37 \mathrm{E}+00$ & & $3.72 \mathrm{E}+00$ \\
\hline $\mathrm{Na}+$ & $2.10 \mathrm{E}+04$ & & $2.32 \mathrm{E}+04$ \\
\hline $\mathrm{Ni}+3$ & & & \\
\hline $\mathrm{Si}+4$ & $3.22 \mathrm{E}+00$ & & $3.55 \mathrm{E}+00$ \\
\hline $\mathrm{Sr}+2$ & $0.00 \mathrm{E}+00$ & & \\
\hline $\mathrm{UO2}+2$ & $3.89 \mathrm{E}+02$ & & $4.29 \mathrm{E}+02$ \\
\hline $\mathrm{Zr}+4$ & $1.75 \mathrm{E}-02$ & & $1.93 \mathrm{E}-02$ \\
\hline $\mathrm{Al}(\mathrm{OH}) 4-$ & $4.44 \mathrm{E}+02$ & & $4.90 \mathrm{E}+02$ \\
\hline $\mathrm{C} 2 \mathrm{O} 4-2$ & $9.64 \mathrm{E}+03$ & & $1.06 \mathrm{E}+04$ \\
\hline $\mathrm{Cl}-$ & $1.13 \mathrm{E}+02$ & & $1.25 \mathrm{E}+02$ \\
\hline $\mathrm{CO} 3-2$ & $1.15 \mathrm{E}+04$ & & $1.27 \mathrm{E}+04$ \\
\hline $\mathrm{Cr}(\mathrm{OH}) 4-$ & $3.46 \mathrm{E}-02$ & & $3.82 \mathrm{E}-02$ \\
\hline $\mathrm{CrO} 4-2$ & $0.00 \mathrm{E}+00$ & & \\
\hline $\mathrm{F}-$ & $2.61 \mathrm{E}+01$ & & $2.88 \mathrm{E}+01$ \\
\hline $\mathrm{HCO}-$ & $3.91 \mathrm{E}+03$ & & $4.31 \mathrm{E}+03$ \\
\hline $\mathrm{NO}-$ & $2.71 \mathrm{E}+03$ & & $2.99 \mathrm{E}+03$ \\
\hline $\mathrm{NO}-$ & $2.84 \mathrm{E}+02$ & & $3.13 \mathrm{E}+02$ \\
\hline $\mathrm{OH}-$ & $2.53 \mathrm{E}+03$ & & $2.79 \mathrm{E}+03$ \\
\hline $\mathrm{PO}-3$ & $6.40 \mathrm{E}+02$ & & $7.06 \mathrm{E}+02$ \\
\hline $\mathrm{SO} 4-2$ & $6.51 \mathrm{E}+02$ & & $7.18 \mathrm{E}+02$ \\
\hline $\mathrm{H} 2 \mathrm{O}$ & $1.18 \mathrm{E}+06$ & $1.57 \mathrm{E}+06$ & $2.88 \mathrm{E}+06$ \\
\hline
\end{tabular}


HNF-SD-TWR-AGA-003

Revision 0

Table C-2. Alternative 2 Material Balance.

(Sheet 12 of 12)

\begin{tabular}{|l|c|c|c|}
\hline STREAM NAME & $18 \mathrm{~B}$ & 19 & 20 \\
\hline SOLED COMPONENTS & & & \\
\hline Total Mass Flow $(\mathrm{kg})$ & $1.02 \mathrm{E}+03$ & & $3.09 \mathrm{E}+05$ \\
\hline
\end{tabular}

Radionuclides (Ci)

\begin{tabular}{|l|l|l|l|}
\hline Pu-239 & $1.15 \mathrm{E}+01$ & & $3.48 \mathrm{E}+03$ \\
\hline Sr-90 & $1.39 \mathrm{E}+04$ & & $4.19 \mathrm{E}+06$ \\
\hline Y-90 & $1.39 \mathrm{E}+04$ & & $4.19 \mathrm{E}+06$ \\
\hline Total Curies & $2.77 \mathrm{E}+04$ & & $8.38 \mathrm{E}+06$ \\
\hline
\end{tabular}

Chemicals $(\mathrm{kg})$

\begin{tabular}{|l|c|l|l|}
\hline $\mathrm{Ag}+$ & $7.96 \mathrm{E}+00$ & & $2.40 \mathrm{E}+03$ \\
\hline $\mathrm{Al}+3$ & $6.74 \mathrm{E}+01$ & & $2.04 \mathrm{E}+04$ \\
\hline $\mathrm{Bi}+3$ & $2.39 \mathrm{E}-02$ & & $7.23 \mathrm{E}+00$ \\
\hline $\mathrm{Ca}+2$ & $1.56 \mathrm{E}+01$ & & $4.71 \mathrm{E}+03$ \\
\hline $\mathrm{Cr}+3$ & $3.37 \mathrm{E}+00$ & & $1.02 \mathrm{E}+03$ \\
\hline $\mathrm{Fe}+3$ & $1.93 \mathrm{E}+02$ & & $5.83 \mathrm{E}+04$ \\
\hline $\mathrm{La}+3$ & $1.86 \mathrm{E}+00$ & & $5.62 \mathrm{E}+02$ \\
\hline $\mathrm{Mg}+2$ & $3.20 \mathrm{E}+00$ & & $9.67 \mathrm{E}+02$ \\
\hline $\mathrm{Mn}+4$ & $9.29 \mathrm{E}+00$ & & $2.81 \mathrm{E}+03$ \\
\hline $\mathrm{Na}+$ & $0.00 \mathrm{E}+00$ & & \\
\hline $\mathrm{Ni}+3$ & $2.92 \mathrm{E}+00$ & & $8.81 \mathrm{E}+02$ \\
\hline $\mathrm{Pb}+4$ & $1.43 \mathrm{E}+01$ & & $4.32 \mathrm{E}+03$ \\
\hline $\mathrm{Pu}+4$ & $1.86 \mathrm{E}-01$ & & $5.61 \mathrm{E}+01$ \\
\hline $\mathrm{Sr}+2$ & $4.98 \mathrm{E}-01$ & & $1.50 \mathrm{E}+02$ \\
\hline $\mathrm{UO} 2+2$ & $0.00 \mathrm{E}+00$ & & \\
\hline $\mathrm{Zr}+4$ & $1.92 \mathrm{E}+00$ & & $5.81 \mathrm{E}+02$ \\
\hline $\mathrm{C} 2 \mathrm{O} 4-2$ & $0.00 \mathrm{E}+00$ & & \\
\hline $\mathrm{Cl}-$ & $0.00 \mathrm{E}+00$ & & \\
\hline $\mathrm{CO}-2$ & $2.52 \mathrm{E}+00$ & & $7.62 \mathrm{E}+02$ \\
\hline $\mathrm{F}-$ & $0.00 \mathrm{E}+00$ & & \\
\hline $\mathrm{OH}-$ & $3.23 \mathrm{E}+02$ & & $9.75 \mathrm{E}+04$ \\
\hline $\mathrm{PO} 4-3$ & $3.94 \mathrm{E}+01$ & & $1.19 \mathrm{E}+04$ \\
\hline $\mathrm{KAlSiO4}$ & $1.36 \mathrm{E}+00$ & & $4.11 \mathrm{E}+02$ \\
\hline $\mathrm{NaAlSiO4}$ & $3.34 \mathrm{E}+02$ & & $1.01 \mathrm{E}+05$ \\
\hline
\end{tabular}


HNF-SD-TWR-AGA-003

Revision 0

\section{APPENDIX D}

\section{HANFORD TANK WASTE OPERATION SIMULATOR RESULTS}


HNF-SD-TWR-AGA-003

Revision 0

This page intentionally left blank. 


\section{APPENDIX D}

\section{HANFORD TANK WASTE OPERATION SIMULATOR RESULTS}

The Hanford Tank Waste Operation Simulator (HTWOS) was used to create an integrated waste transfer schedule, which incorporates high-level waste (HLW) pretreatment processing Alternatives 1 (enhanced sludge washing) and 2 (sludge washing). The HTWOS model was used to determine if enough double-shell tank (DST) space exists to accomplish . either alternative, without limiting the ability to deliver a continuous HLW feed to the Private Contractor, starting June 1,2002. Since Alternatives 1 and 2 utilize the greatest and least amount of DST space, respectively, Alternatives 3 through 8 were not modeled, since the two extremes are already represented by the two cases.

Tables D-1 and D-2 contain the projected transfers for Alternatives 1 and 2, respectively. Only the transfers pertinent to HLW pretreatment and feed staging are annotated. The waste transfer information contained in these tables comes directly from the HTWOS model. The model assumptions are provided in Kirkbride et al. (1997). The results indicate that enough DST space exists to accomplish either alternative, with no impact on the ability to deliver a continuous HLW feed. 
HNF-SD-TWR-AGA-003

Revision 0

This page intentionally left blank. 
HNF-SD-TWR-AGA-003

Revision 0

Table D-1. Pretreatment Processing Alternative 1 -

Projected Transfers Through April 2011 (10 sheets).

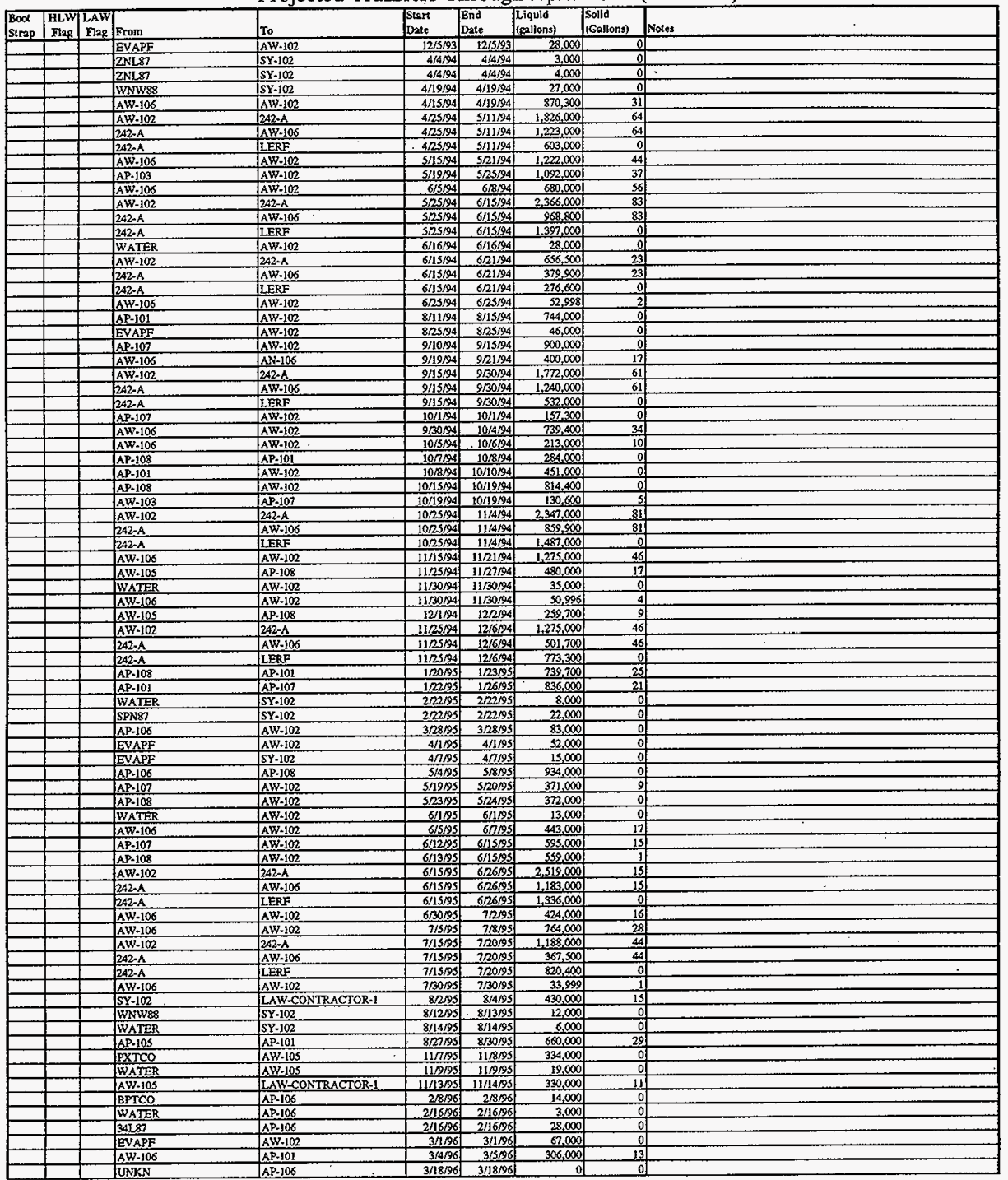


HNF-SD-TWR-AGA-003

\section{Revision 0}

Table D-1. Pretreatment Processing Alternative 1 -

Projected Transfers Through April 2011 (10 sheets).

\begin{tabular}{|c|c|c|c|c|c|c|c|c|c|}
\hline $\begin{array}{l}B o \alpha \\
\text { Strop }\end{array}$ & \begin{tabular}{|l|} 
HLW \\
Flag
\end{tabular} & \begin{tabular}{|l|} 
LAw \\
Flag
\end{tabular} & Fron & To & $\begin{array}{l}\text { Start } \\
\text { Date }\end{array}$ & $\begin{array}{l}\text { End } \\
\text { Date }\end{array}$ & $\begin{array}{l}\text { Ligquid } \\
\text { iglions) }\end{array}$ & $\begin{array}{l}\text { Solid } \\
\text { (Gallons) }\end{array}$ & Noles \\
\hline & & & WATER & AP-106 & $3 / 18 / 96$ & $3 / 1 / 8 / 96$ & 28,000 & 0 & \\
\hline & & & BPTCO & AP.106 & $3 / 20 / 96$ & 320096 & 25,000 & 0 & \\
\hline & & & EVAPF & AW-102 & $4 / 1196$ & $4 / 1 / 96$ & 22,000 & 0 & \\
\hline & & & EVAPF & AW. 106 & $4 / 1 / 96$ & $4 / 1 / 196$ & 14,000 & 0 & \\
\hline & & & WWESS & $5 Y-102$ & $3 / 2096$ & $4 / 2 / 296$ & 169,000 & $\overline{0}$ & \\
\hline & & & WATER & $5 Y \cdot 102$ & $4 / 13 / 96$ & $4 / 13 / 96$ & 49,000 & 0 & \\
\hline & & & SPNB7 & AP-106 & $4 / 17 / 96$ & $4 / 177 / 96$ & 11,000 & 0 & \\
\hline 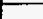 & & 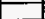 & SPN87 & AP-106 & $4 / 17 / 96$ & $4 / 17 / 96$ & 11,000 & 0 & \\
\hline & & & BPTCO & AP. 108 & $4 / 19 / 96$ & $4 / 19 / 96$ & 14.000 & 0 & \\
\hline & & & WATER & AP-108 & $4 / 19 / 965$ & $4 / 19 / 95$ & 3,000 & 0 & \\
\hline . & & . & AW-106 & $A W-102$ & $5 / 6196$ & $5 / 6 \% 96$ & 28,999 & 1 & \\
\hline & & & AP-104 & AW-102 & $5 / 6 / 96$ & $5 / 10 / 96$ & 812,000 & 0 & \\
\hline & & & AW-102 & $242-A$ & $5 / 1590$ & $5 / 20196$ & $1,019,000$ & 35 & \\
\hline & & & $242-\mathrm{A}$ & Aw-106 & $5 / 15 / 96$ & $5 / 20 / 96$ & 131,700 & 35 & \\
\hline & & & $242-A$ & LERF & $5 / 15 / 96$ & $5 / 20 / 96$ & 887,300 & 0 & \\
\hline & & & EVAPF & AW.102 & $5 / 50 / 96$ & 5.5096 & 3,000 & 0 & \\
\hline & & & AW-105 & AW-IO2 & $5 / 30 / 96$ & $5 / 30 / 96$ & 61,997 & 3 & \\
\hline & & & TAL 88 & AP.106 & $6 / 6 / 96$ & $6 / 6996$ & 10,000 & 0 & \\
\hline & & & PXTCO & Aw-105 & $6 / 30,96$ & $6 / 30 / 96$ & 95,000 & 0 & \\
\hline & & & WATER & AP-10S & $7 / 1196$ & $7 / 1 / 96$ & 1,000 & 0 & \\
\hline & & & WATER & AY 102 & $8 / 15195$ & $8 / 15 / 96$ & 27,000 & 0 & \\
\hline & & & AN-101 & AP.105 & $8116 \% 5$ & $8 / 20 / 96$ & 928,800 & 0 & \\
\hline & & & AW-106 & AP. 101 & 19197 & $1 / 9 / 97$ & 69.511 & 3 & \\
\hline & & & AN-106 & $\mathrm{AP}-108$ & $1 / 16,97$ & $1 / 16 / 97$ & 177,000 & 7 & \\
\hline & & & AP+105 & AW-102 & $2 / 1092$ & $2 / 14 / 997$ & 942,000 & 42 & \\
\hline & & & AW-102 & $242-A$ & 2260997 & $3 / 2,97$ & 977,500 & 43 & \\
\hline & & & $242 . \mathrm{A}$ & AW.106 & $2 \pi 6597$ & $3 / 2 / 97$ & 576,400 & 43 & \\
\hline & & & $242-A$ & LERF & 2206997 & $3 / 2 / 97$ & 401,100 & 0 & \\
\hline$Y$ & $\mathrm{Y}$ & $\bar{Y}$ & AY. 101 & AP: 108 & $3 / 3 / 97$ & $3 / 7 / 97$ & 800,000 & 30 & Empoy 1AY \\
\hline & & & AW-106 & $A P+10 S$ & $4 / 3 / 97$ & $4 / 3 / 27$ & 576,200 & 29 & \\
\hline & & & $A \mathrm{~N}=108$ & AP-108 & $7 / 1 / 97$ & $7 \pi / 97$ & 194,000 & 7 & \\
\hline & & & AP-108 & AW-102. & $7 / 3 / 97$ & $7 / 10 / 97$ & $1,198,000$ & 43 & \\
\hline & & & $A W-102$ & $242-\mathrm{A}$ & $7 / 5 / 97$ & $7 / 10997$ & $3,198,000$ & 40 & \\
\hline & & & $242-A$ & AW-10.S & $7 / 5 / 97$ & 7/1092 & 346.700 & 40 & \\
\hline & & & $242-A$ & LERF & 75197 & $7 / 10097$ & 851,300 & 0 & \\
\hline & & & ZNL87 & $S Y-102$ & 73197 & 731,197 & 2,000 & 0 & \\
\hline & & & WATER & $5 Y-102$ & $7 / 31 / 97$ & $7 / 31997$ & 62,000 & 0 & \\
\hline & & & WNWB8 & $S Y-102$ & $7 / 3197$ & $8 / 1 / 197$ & 223,000 & 0 & \\
\hline & & & AW-10S & AN-106 & $8 / 5 / 97$ & $8 / 6 / 97$ & 346,400 & 16 & \\
\hline & & & $5 Y-102$ & AP-107 & $8 / 15 / 97$ & $8 / 17 / 97$ & 520,000 & 20 & \\
\hline & & & WASH-CAUSTIC & $A N-107$ & 10,297 & $10 / 297$ & 66,000 & 0 & \\
\hline & & & AY-102 & AP-106 & $10 \pi / 97$ & $100 / 92$ & 35,999 & I & \\
\hline & & & WATER & AY.102 & 10889 & 10809 & 176,000 & 0 & \\
\hline - & & & 2N1L87 & SY-102 & $11 / 30197$ & $11 / 30 / 97$ & 4,000 & 0 & \\
\hline & & & SPNS7 & SY.102 & $11 / 3009$ & 1130197 & 7,000 & 0 & \\
\hline & & & WSTER & $5 Y-102$ & $11 / 30 / 97$ & $11 / 30 / 97$ & 112,000 & 0 & \\
\hline & & & WWW & $5 Y \cdot 102$ & $11 / 30 / 97$ & $12 / 2997$ & 422,000 & 0 & \\
\hline & & & TNSB8 & AP-106 & $12 / 5 / 97$ & $12 / 5 / 92$ & 1,500 & 500 & \\
\hline & & & PXTCO & AP-106 & 1215597 & 1215097 & 10,000 & 0 & \\
\hline & & & WESF & AP-106 & $12 / 5 / 97$ & $12 / 5197$ & 25,000 & 0 & \\
\hline & & & TAI 88 & AP.106 & $12 / 5,97$ & $12 / 51927$ & 20,000 & 0 & \\
\hline & & & SPN87 & AP-106 & $12 / 5597$ & $12 / 5 / 97$ & 25,000 & 0 & \\
\hline & & & 34287 & AP-106 & $12 / 5 / 97$ & $12 / 5 / 97$ & 67,000 & 0 & \\
\hline & & & WATER & AP-106 & $12 / 5 / 97$ & $12 / 5992$ & 109,000 & 6 & \\
\hline & & & EVAPF & $A P-106$ & $12 / 5 / 97$ & $12 / 5 / 92$ & 150,000 & 0 & \\
\hline & & & BPTCO & AP.106 & $12 / 5,97$ & $12 / 5997$ & 136,000 & 0 & \\
\hline & & & WNDS8 & AP-106 & $12 / 5197$ & $1216 / 2$ & 204,000 & 0 & \\
\hline & & & $5 Y \cdot 102$ & AP-107 & 121.597 & $12 / 17979$ & 520,000 & 21 & \\
\hline & & & AP-106 & $\mathrm{AP}-104$ & $12 / 20 / 97$ & $12 / 25 / 93$ & 996.800 & 35 & \\
\hline & & & SPNB7 & $\$ Y Y-102$ & $2 / 28,98$ & 228898 & 6,000 & 0 & \\
\hline & & & WATER & SY.102 & 222898 & 228898 & 103,000 & 0 & \\
\hline & & & WNW's & $\$ Y .102$ & 228998 & $3 / 2 / 98$ & 410,000 & 0 & \\
\hline & & & $\mathrm{AP}+104$ & $A W-102$ & $3 / 1 / 98$ & $3 / 6 / 98$ & 995,300 & 34 & \\
\hline & & & $5 Y-102$ & AP.104 & $3 / 15 / 98$ & $3 / 1798$ & 520,000 & 21 & \\
\hline & & & $\mathrm{AW}-102$ & $242 \cdot A$ & $4 / 5 / 98$ & 49098 & 995,300 & 35 & \\
\hline & & & $242 \cdot A$ & AW-106 & $4 / 3 / 98$ & $4 / 9 / 98$ & 168,500 & 35 & \\
\hline & & & $242-A$ & LERF & $4 / 5 / 98$ & 49908 & 825,800 & 0 & \\
\hline & & & WATER & AP.107 & $4 / 10998$ & $4 / 1009$ & 70,000 & 0 & \\
\hline & & & AP-107 & AW-102 & $4 / 25 / 98$ & $5 \pi / 98$ & $1,104,000$ & 41 & \\
\hline & & & $A W-102$ & $242 \cdot A$ & $5 / 5 / 98$ & $5 / 1098$ & $1,104,000$ & 39 & \\
\hline & & & $242-A$ & $\longdiv { A W = 1 0 6 }$ & $5 / 3 / 98$ & $5 / 1008$ & 441,000 & 39 & \\
\hline & & & $242 \cdot A$ & LERF & $5 / 5 / 98$ & $5 / 10 / 98$ & 663,000 & 0 & \\
\hline & & & SPN87 & $S Y \cdot 102$ & 5,51198 & $5 / 3198$ & 6,000 & 0 & \\
\hline & & & WCWB8 & SY-102 & $5 / 31 / 98$ & $5 / 31 / 98$ & 38,000 & 0 & \\
\hline & & & WATER & $S Y \cdot 102$ & $5 \sqrt[3108]{108}$ & $5 / 3198$ & 119,000 & 0 & \\
\hline & & & WNWES & $5 Y-102$ & $5 / 31 / 98$ & 6.2198 & 435,000 & 0 & \\
\hline & & & $A W=106$ & AP.10S & $6,3 / 989$ & $6 / 4 / 98$ & 341,000 & 16 & \\
\hline & & & SY-102 & AP-104 & $6 / 15 / 98$ & $6 / 17 / 98$ & 520,000 & 21 & \\
\hline & $\mathbf{Y}$ & & $A Y=102$ & $\mathrm{c}-106$ & $7 / 1 / 98$ & $7 / 5 / 98$ & 920,700 & 31 & Tor C-106 Rerrieval - Lumpod \\
\hline & & & AP-10! & $A W+103$ & $8 / 6 / 98$ & $8 / 9198$ & 623,000 & 28 & \\
\hline
\end{tabular}


HNF-SD-TWR-AGA-003

Revision 0

Table D-1. Pretreatment Processing Alternative 1 -

Projected Transfers Through April 2011 (10 sheets).

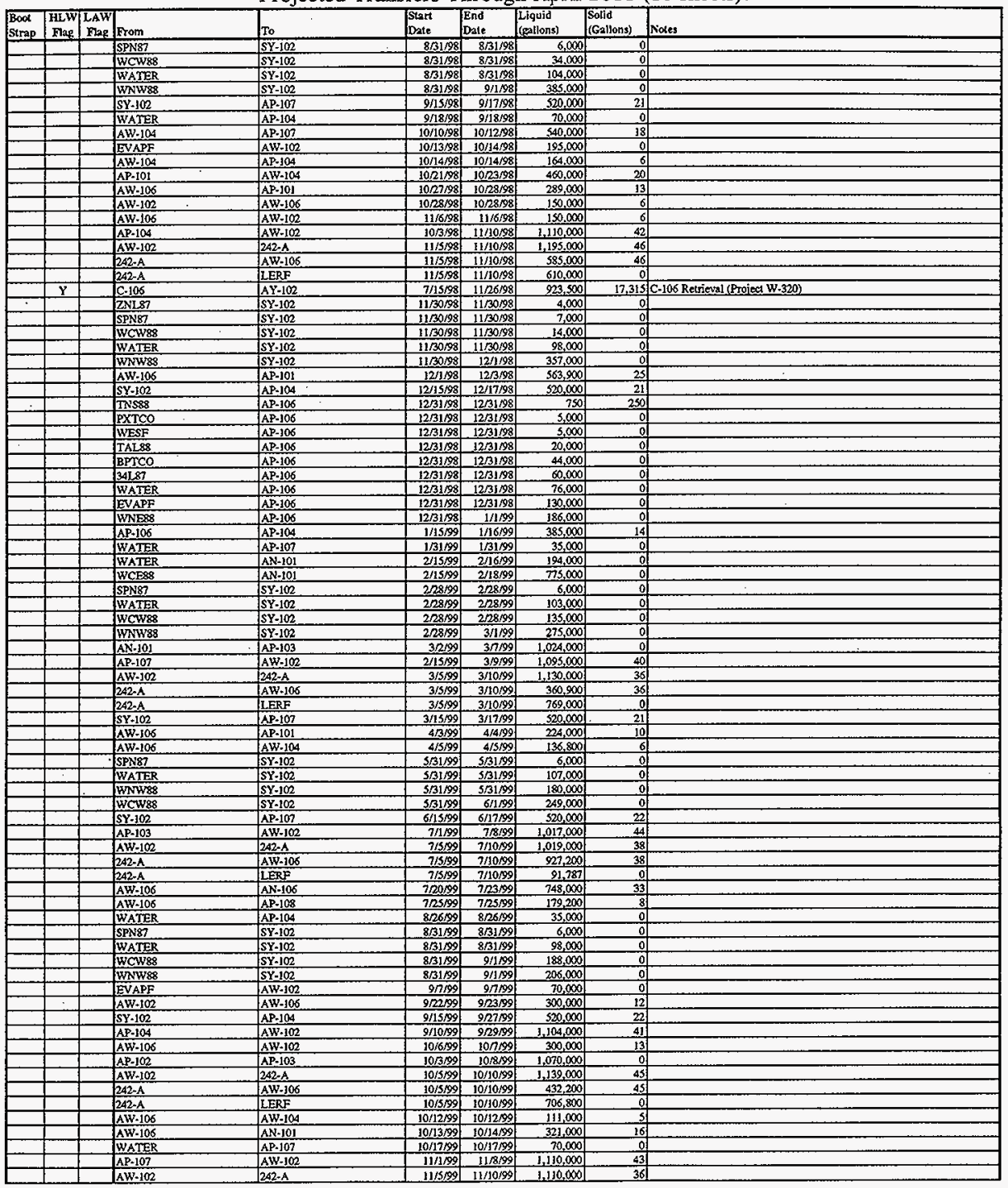

D-7 
HNF-SD-TWR-AGA-003

\section{Revision 0}

Table D-1. Pretreatment Processing Alternative 1 -

Projected Transfers Through April 2011 (10 sheets).

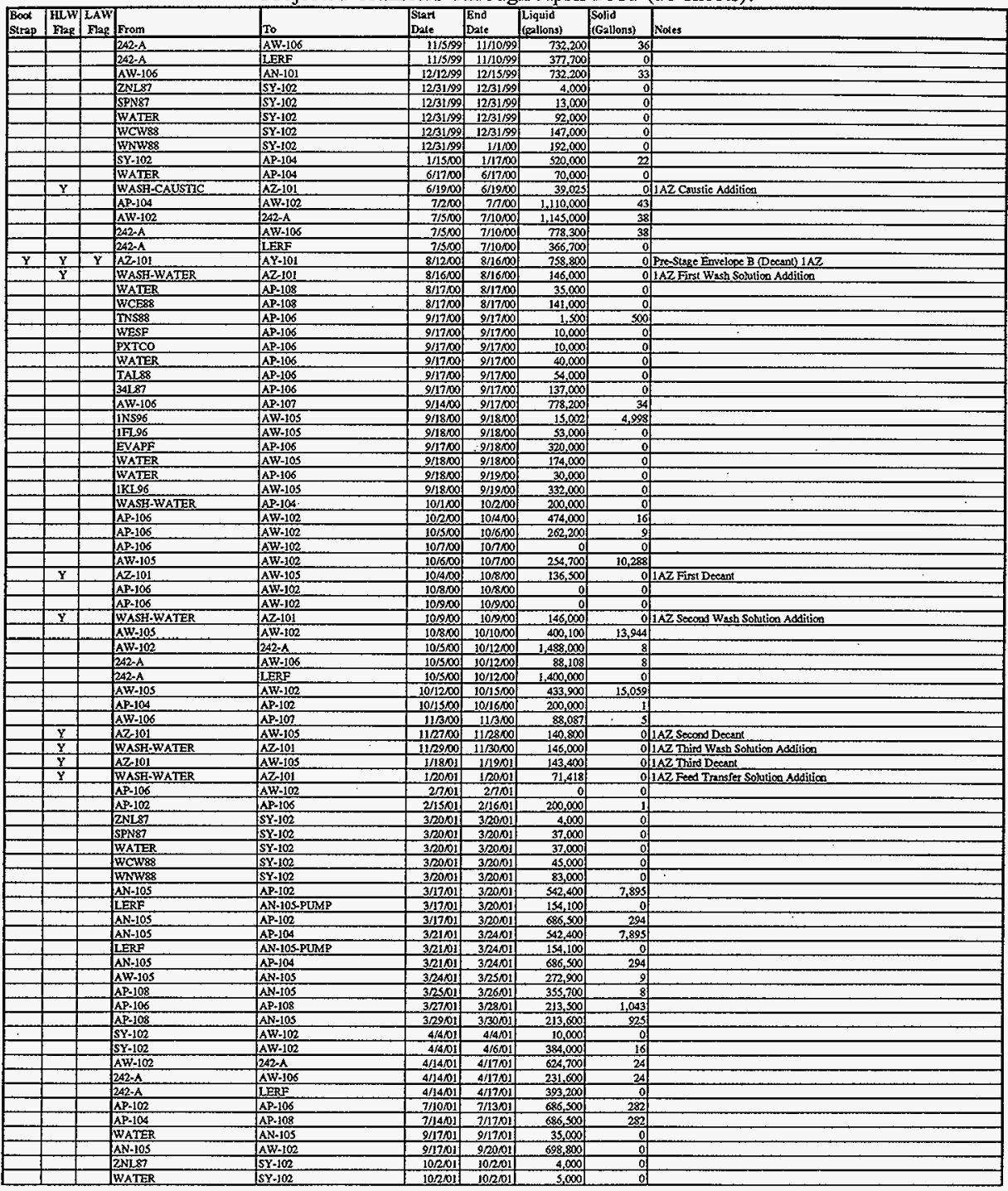




\section{HNF-SD-TWR-AGA-003 \\ Revision 0}

Table D-1. Pretreatment Processing Alternative 1 -

Projected Transfers Through April 2011 (10 sheets).

\begin{tabular}{|c|c|c|c|c|c|c|c|c|c|}
\hline $\begin{array}{l}\text { Bood } \\
\text { Strap }\end{array}$ & FlW & $\begin{array}{c}\text { LAFY } \\
\text { Flag }\end{array}$ & From & To & $\begin{array}{l}\text { Stant } \\
\text { Date }\end{array}$ & End & $\begin{array}{l}\text { Liguld } \\
\text { (pallons) }\end{array}$ & $\begin{array}{l}\text { Solic } \\
\text { (Gallons) }\end{array}$ & Noles \\
\hline & & & SPN87 & $S Y=102$ & $10 / 2,1$ & 10201 & 25,000 & 0 & 5 \\
\hline & & & $A N-104$ & AP-102 & $10 / 1 / 01$ & $10 / 4 / 01$ & 502,200 & $12,5,13$ & \\
\hline & & & LERF & AN-104-PUMP & $10 / 101$ & $10 / 4 / 01$ & 200,800 & 0 & of \\
\hline & & & AN-104 & AP. 102 & $10 / 101$ & $10 / 4,01$ & 684,700 & 702 & \\
\hline & & & AN-104 & AP-10S & $10 / 501$ & 10801 & 502,200 & 12,513 & \\
\hline & & & LERF & AN-104-PUMP & $10 / 5 / 01$ & $10 / 201$ & 200.800 & 0 & ) \\
\hline & & & AN-104 & AP-104 & $10 / 5 / 01$ & $10 / 3 \times 1$ & 684,700 & 702 & \\
\hline & & & WATER & AW.102 & $10 / 19 \% 1$ & 1] $10 / 19 / 01$ & 35,000 & 0 & t \\
\hline$\cdot$ & & & EVAPF & AW 102 & $10 / 19 / 1$ & $10 / 1901$ & 93,000 & 0 & \\
\hline & $\mathbf{Y}$ & & WASH.CAUSTIC & $A Z-102$ & 112001 & $11 / 20 / 01$ & 20,931 & 0 & 2AZ Canstic Addition \\
\hline & & & AP.107 & AN-104 & $1 / 1,02$ & $1 / 4 / 02$ & 866,800 & 39 & \\
\hline $\mathbf{Y}$ & $Y$ & $\mathbf{Y}$ & AZ, 102 & AY-101 & $1 / 13 / 22$ & $1 / 14,02$ & 130,200 & 0 & 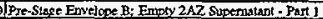 \\
\hline $\mathbf{Y}$ & $\mathrm{Y}$ & & $A Z 102$ & AN-104 & $1 / 14 / 02$ & $1 / 15 / 02$ & 246,100 & 0 & Empty $2 A 7$ Supernatant + Part 2 \\
\hline $\mathbf{Y}$ & $\mathbf{Y}$ & & $A Z-102$ & AN-105 & $1 / 15 / 02$ & $1 / 1502$ & of & 0 & Empty $2 A 2$ Supematant - Part 3 \\
\hline & $\mathbf{Y}$ & & WASH-WATER & AZ-102 & $1 / 15 / 02$ & $1 / 18,02$ & 638,000 & 0 & 2AZ First Wash Solution Adtition \\
\hline & $\mathbf{Y}$ & & AT-102 & AN-105 & $3 / 8 / 02$ & $3 / 11 / 2$ & 618,000 & 0. & $2 \wedge z$ First Decont \\
\hline & $\vec{Y}$ & & WASF-WATER & $A Z-102$ & $3 / 1202$ & $3 / 16 / 02$ & 638.000 & 0 & 2 AZ Second Wash Solution Addition \\
\hline & & & AW-102 & $242-1$ & $3 / 20 / 2$ & $3 / 24 / 02$ & 827,100 & 3] & $\therefore$ \\
\hline & & & $242-\mathrm{A}$ & AW-106 & $3 / 20,02$ & $3 / 24 / 02$ & 275,500 & 31 & \\
\hline & & & $242-\Lambda$ & LERF & $3 / 20 / 02$ & $3 / 24 N 2$ & $5 \$ 1,000$ & 0 & \\
\hline & & & EVAPF & AW.102 & $3 / 26 / 02$ & $3 / 27,02$ & 132,000 & 0 & f \\
\hline & & & AN-105 & AW-102 & $3 / 25 / 02$ & $3 / 29 / 02$ & 695,100 & 0 & of \\
\hline & $\mathbf{Y}$ & & $A Z-102$ & $A N-105$ & $5 / 4 / 02$ & $S \pi / N 2$ & 633,400 & 0 & $2 A 2$ Second Decant \\
\hline & $\mathbf{Y}$ & & WASH-WATER & $A 2102$ & $5 / 8102$ & $5 / 9 / 02$ & 156,200 & 0 & 2 A2 Foed Transfer Sointion Addition \\
\hline & $\bar{Y}$ & & $A Z-101$ & PIHLW FEED TANK & $5 / 1702$ & $5 / 17,02$ & 94,007 & 4,349 & Deliver Esyelooe D, Batch L (laZ First Baleh) \\
\hline & & & WESF & AN-10S & $9 / 1702$ & $9 / 1702$ & 5,000 & 0 & \\
\hline & & & WATER & AN-105 & $9 / 17 / 02$ & $2 / 17102$ & 27,000 & 0 & \\
\hline & & & INSS8 & AN-105 & $9 / 17 / 02$ & $9 / 1702$ & 750 & 250 & \\
\hline & & & TAL 88 & AN-105 & $9 / 17 / 02$ & $9 / 17 / 102$ & 24,000 & 0 & \\
\hline & & & WATER & $\mathrm{AN}-105$ & $9 / 17 / 02$ & $9 / 17 / 102$ & 35,000 & 0 & \\
\hline & & & $34 L 87$ & AN-10S & $9 / 17 / 02$ & $9 / 17 / 02$ & 50,000 & 0 & \\
\hline & & & EVAPF & $A N-105$ & $9 / 17 / 02$ & 2/17N02 & 120,000 & 0 & \\
\hline & & & Aw-102 & $242 \mathrm{~A}$ & $9 / 26,02$ & $9 / 30 / 02$ & 827,300 & 29 & \\
\hline & & & $242-A$ & AW-106 & 926,02 & $9 / 3002$ & 134,600 & 29 & \\
\hline & & & $242-A$ & LERF & $9 / 26,02$ & $9 / 30 / 02$ & 692,900 & 0 & \\
\hline & & & AN-10S & AW-102 & $10 / 2 / 22$ & $10 / 5 / 102$ & 699,5001 & 0 & \\
\hline & $\mathbf{Y}$ & & PIALW FEED TANK & HLW.CONTRACTOR & $6 / 1 / 02$ & 113002 & 94,007 & 4,349 & Process Enveloge D Balch 1 \\
\hline & $\mathbf{Y}$ & & AZ:101 & PIRLW FEEO TANK & $11 / 30 \mathrm{~N}_{2}$ & $12 / 1 / 02$ & 94,007 & 4,349 & Delive Equelope D, Batch 2 (1 A? Second Ratch) \\
\hline & & & RETURN 1 & AP-107 & $12 / 3102$ & $12 \sqrt[3 / 02]{ }$ & 8,705 & 295 & \\
\hline & & & RETURN 2 & $A \cdot \cdot 10$ ? & $1 / 203$ & $1 / 2 / 03$ & 8,669 & 33! & \\
\hline & & & AP-102 & AP: 106 & $1 / 5,03$ & $19 / 03$ & 684,700 & 886 & \\
\hline & & & AP.104 & AP. 108 & $1 / 9 / 03$ & $1 / 12 / 83$ & 684,700 & 686 & \\
\hline & & & AW.101 & $\mathrm{AP}-102 \cdot$ & $1 / 103$ & $1 / 13 / 03$ & 537,200 & 13,013 & \\
\hline & & & LERE & AW-101-PUMP & $1 / 9103$ & $1 / 13 / 03$ & 308,100 & 0 & \\
\hline & & & AW-101 & AP-102 & $1 / 9,03$ & $1 / 13 / 03$ & 845,400 & 13,013 & \\
\hline & & & AW-101 & AP:104 & $1 / 14 / 03$ & $1 / 18 / 03$ & 337,200 & $13,0,3$ & \\
\hline & & & LERF & AW-101-PUMP & $1 / 1403$ & $1 / 18 / 03$ & 308.100 & 0 & \\
\hline & & & AW-101 & AP.j04 & $1 / 14 / 03$ & $1 / 18 / 03$ & 845,400 & 13.013 & \\
\hline & & & ZNLS7 & $s x-102$ & $3 / 20 / 03$ & 320003 & 3,000 & 0 & \\
\hline & & & WATER & $5 \mathrm{SY} \cdot 102$ & $3 / 20 / 03$ & $3 / 2003$ & 6,000 & 0 & \\
\hline & & & SPN87 & SY-102 & $3 / 20 / 03$ & $3 / 20 / 03$ & 25,000 & 0 & \\
\hline & & & $5 Y+102$ & AW-102 & $4 / 4,03$ & $4 / 4 / 43$ & 29,999 & 1 & \\
\hline & & & $S Y+102$ & $A W-102$ & $4 / 4,03$ & $4 / 4 / 03$ & 4,000 & 0 & \\
\hline & & & $A B=102$ & $242 . \mathrm{A}$ & $4 / 4 / 03$ & $4 / 803$ & 733,900 & 25 & \\
\hline & & & $242-A$ & $A W-106$ & $4 / 4 / 03$ & $4 / 8 / 03$ & 48,669 & 25 & \\
\hline & & & $242-A$ & LERF & $4 / 4 / 03$ & $4 / 8 / 03$ & 685,200 & 0 & \\
\hline & & & $A W-106$ & $A W_{-102}$ & $4 / 8103$ & $4 / 12 / 13$ & $689,700)$ & 32 & \\
\hline & & & RETURN 1 & AP= 107 & $4 / 28 / 03$ & $4 / 28,03$ & 8,679 & 325 & \\
\hline & & & REIURN 2 & AP-107 & $5 / 2203$ & $5 / 23 / 03$ & 8,672 & 332 & \\
\hline & $Y$ & & P1HLW FEED TANK & ALW-CONTRACTOR & $12 / 102$ & $6 / 203$ & 94,007 & 4,349 & Process Fnvelope D Bater 2 \\
\hline & $\boldsymbol{Y}$ & & 12,102 & PIHLW FEED TANK & $6 / 2103$ & $6 / 2 / 03$ & 101,900 & 3,856 & Deliver Envelope D, Batch 3 (2A) Firs (Batch) \\
\hline & & & RETURN 1 & AP $=107$ & $9 / 1203$ & $9 / 1203$ & 8.674 & 332 & \\
\hline & & & TNSS8 & AN-10S & $9 / 17 / 03$ & $9 / 17 / 03$ & 750 & 250 & \\
\hline & & & WESF & AN-105 & $9 / 17 / 03$ & $9 / 17 / 03$ & 5,000 & 0 & \\
\hline & & & TAIS8 & AN-10S & $9 / 17 / 03$ & $9 / 17 / 03$ & $24, \infty 00$ & (0) & \\
\hline & & & WATER & $\mathrm{AN}-105$ & $9 / 17 / 03$ & $9 / 17 / 03$ & 35,000 & 0 & \\
\hline & & & 34 LS7 & AN.10S & $9 / 17 / 03$ & $9 / 17 / 03$ & 37,000 & a) & \\
\hline & & & WATER & AN-10S & $9 / 17 / 103$ & $9 / 17 / 03$ & 8,000 & 0 & \\
\hline & & & EVAPF & AN.10S & $9 / 17 / 03$ & $9 / 17 / 03$ & 120,000 & 0 & \\
\hline & & & WATER & $A N-105$ & $9 / 18,03$ & $9 / 18 / 03$ & 22,000 & of & \\
\hline & & & EVAPF & Aw-102 & 9,20003 & $9 / 20 / 03$ & 35,000 & 0 & \\
\hline & & & $A N-105$ & $A W=102$ & $9 / 20 / 03$ & $9 / 3,03$ & 548,700 & 0 & \\
\hline & & & AP-102 & $A P-106$ & $9 / 29 / 03$ & $10 / 2,03$ & 740,300 & 1,581 & \\
\hline & & & RETURN 2 & AP*107 & $10 / 6 / 03$ & $10 / 6,03$ & 8.669 & 332 & \\
\hline & & & AP-104 & AP-108 & $10 / 2 / 03$ & $10 / 6,03$ & 740,300 & 1,581 & \\
\hline & & & $A N=103$ & AP. 102 & $10 / 3 / 03$ & $10 / 6 / 03$ & 436,600 & 28,133 & \\
\hline & & & LERF & AN-103-PUMP & $10 / 3 / 03$ & $10 / 6,03$ & 264,900 & of & \\
\hline & & & AN-103 & $A P_{-102}$ & $10 / 3 \times 3$ & $10 / 6,03$ & 738,300 & 549 & \\
\hline & & & $A N+103$ & AP.104 & $10 / 703$ & $10 / 1003$ & 436,600 & 28,133 & \\
\hline & & & LERF & AN-103.PUMP & $10 \pi / 03$ & $10 / 2003$ & 204,900 & of & \\
\hline
\end{tabular}


HNF-SD-TWR-AGA-003

\section{Revision 0}

Table D-1. Pretreatment Processing Alternative 1 -

Projected Transfers Through April 2011 (10 sheets).

\begin{tabular}{|c|c|c|c|c|c|c|c|c|c|}
\hline $\begin{array}{l}000 \\
\text { strap }\end{array}$ & $\begin{array}{l}\text { HLw } \\
\text { Elag }\end{array}$ & \begin{tabular}{|l} 
LAW \\
Fag
\end{tabular} & From & To & $\begin{array}{l}\text { Stratt } \\
\text { Date }\end{array}$ & $\begin{array}{l}\text { End } \\
\text { Dote }\end{array}$ & $\begin{array}{l}\text { Liquid } \\
\text { (gallons) }\end{array}$ & $\begin{array}{l}\text { Solid } \\
\text { (Gallons) }\end{array}$ & Notes \\
\hline & & & AN-103 & AP-104 & $10 / 203$ & $10 / 10 / 03$ & 738,300 & 549 & \\
\hline & $y$ & & WASH-CAUSTIC & $A Y-102$ & $10 / 19003$ & $10 / 19 / 03$ & 39,233 & 02 & 2AY Cautic Aclition \\
\hline & & & RETURN 1 & AP-10? & 11/14403) & $11 / 34 / 03$ & 8.075 & 330 & \\
\hline & & & REIURN 2 & $A P=107$ & $11 / 25 \mathrm{s03}$ & 112503 & 8,675 & 330 & \\
\hline & $\mathbf{Y}$ & & $A Y-102$ & $A N-205$ & $12 / 12 / 03$ & $12 / 15 / 03$ & 730,400 & 02 & 2AY Initial Decant \\
\hline & $\mathbf{Y}$ & & WASH-WATER & $A Y=102$ & $12 / 15,03$ & $12 / 1703$ & 325,000 & 0 & 2AY First Wush Sondion Addition \\
\hline & $Y$ & & PIHLW FEED TANK & ALW-CONTRACTOR & 6,203 & $1 / 2 / 04$ & 101,500 & 3,856 & Process Envelope D Batch 3 \\
\hline & $\mathbf{Y}$ & & AZ-102 & PIFLW FEED TANK & $15 / 04$ & $1 / 3 / 04$ & 101,500 & 3,856 & Detiver Envelope D Rstech 4 (2 A 2 Socond Batch) \\
\hline & & & RETURN 1 & AP-107 & $1 / 304$ & $1 / 304$ & 8.674 & 330 & \\
\hline & & & RETURN 2 & AP.10? & $1 / 4,04$ & $1 / 14 / 04$ & 8.673 & 330 & \\
\hline $\mathbf{Y}$ & $\mathbf{Y}$ & & $A Y-102$ & $A N^{2}-105$ & $2 / 4,04$ & $2 / 5 / 04$ & 168,000 & of & 2AY First Decant - Pett 3 \\
\hline $\bar{Y}$ & $\mathrm{Y}$ & & AY-102 & AW-10S & $2 / 5,04$ & $2 / 6009$ & 147,200 & 0 & 2AY First Decant - Parl 2 \\
\hline & $\bar{Y}$ & & WASH.WATER & AY $=102$ & $2 n 104$ & $2 / 204$ & 325,000 & 0 & 2AY Second Wash Solution Addilion \\
\hline & & & RETURN 1 & AP-107 & $2 / 2204$ & $2 / 22 / 04$ & 8,674 & 330 & \\
\hline & & & RETURN 2 & AP.10? & $3 / 3 / 04$ & 3,304 & 8.673 & 330 & \\
\hline & & & WATER & SY-102 & 320,04 & 3,2004 & 5,000 & a) & \\
\hline & & & $2 \mathrm{~N} 187$ & $S Y=102$ & $3 / 20104$ & $3 / 20 / 09$ & 3,000 & 0 & \\
\hline & & & SPN87 & $5 Y-102$ & $3 / 2004$ & 3,20004 & 25,000 & 0 & \\
\hline & & & $A W-102$ & $242, A$ & $3 / 23 / 04$ & 32504 & 584,000 & 20 & \\
\hline & & & $242-A$ & $A w-106$ & $3 / 23 / 04$ & $3 / 25 / 04$ & 22,176 & 20 & \\
\hline & & & $242 \times 1$ & LERF & $3 / 23 / 04$ & $3 / 2509$ & 561,8000 & of & \\
\hline & & & AN+105 & AW-102 & 326104 & 32904 & 698,000 & 1,349 & \\
\hline & $\bar{Y}$ & & $A Y+102$ & AN-105 & $3 / 28 / 24$ & $3 / 3004$ & 320,800 & of & 2AY Second Decant \\
\hline & & & AW-105 & AN-105 & 35004 & $3 / 30 / 04$ & 147,200 & 0 & \\
\hline & $Y$ & & WASH-WATER & $\mathrm{AY} \cdot 102$ & $3 / 3104$ & $4 / 204$ & 365,400 & of & 2AY Fool Transfer Sohution Addition \\
\hline & & & $S Y=102$ & AW-102 & $4 / 4,04$ & $4 / 4 / 04$ & 3,040 & 0 & \\
\hline & & & SY -102 & AW-102 & $4 / 4 / 04$ & $4 / 4 / 04$ & 29,999 & 1 & \\
\hline & & & RETURN I & AP-10T & $4 / 13 / 24$ & $4 / 1304$ & 8,674 & 330 & \\
\hline & & & RETURN 2. & AP-107 & $4 / 22104$ & $4 \sqrt{2204}$ & 8,673 & 330 & \\
\hline & & & AP-102 & AP-106 & $5 / 3,04$ & 57704 & 753,200 & 1,687 & \\
\hline & & & WASH-WATER & $A^{P}-102$ & $5 / 804$ & $5 / 804$ & 118,100 & 0 & \\
\hline & & & AP-104 & AP-108 & $5 / 704$ & $5 / 10 / 24$ & 753,200 & 1,687 & \\
\hline & & & WASH.WATER & AP-104 & $5 / 1104$ & $5 / 1104$ & 118,100 & 0 & \\
\hline & & & AP 102 & AN-105 & $5 / 1209$ & $5 / 1304$ & 209,300 & 9.168 & \\
\hline & & & AP-104 & AN-10S & $5 / 14 / 04$ & $5 / 1504$ & 201,400 & 8.816 & \\
\hline & & & AP 104 & AW-10S & 5/1504 & 5/15/04 & 8,045 & 352 & \\
\hline & & & $A Y+101$ & AP-102 & S/15nos & $5 / 1604$ & 256,200 & 10 & \\
\hline & & & AY -101 & AP-104 & 5/17/R4 & $5 / 18,04$ & $25,3,200$ & 10 & \\
\hline & & & Ax-103 & $A N=103$ & 111004 & $5 / 28 / 04$ & 312,500 & 2,846 & \\
\hline & & & RETURN I & $A P-107$ & $6 / 504$ & $6 / 5 / 04$ & 8,673 & 331 & \\
\hline & & & RETURN 2 & $A P+107$ & $6 / 15 / 04$ & 6,1604 & 8,682 & 3311 & \\
\hline$\dot{1}$ & & & RETURN 1 & AP-107 & $8 / 104$ & $8 / 104$ & 8,681 & 331 & \\
\hline & $Y$ & & PIHLW FEED TANK & HLW-CONTRACTOR & $1 / 30 \mathrm{~A}$ & 8404 & 101,900 & 3,856 & Dopeess Envelope D Balch 4 \\
\hline & $y$ & & $A 2,102$ & PIHLW FEPD TANK & $8 / 4 / 04$ & $8 / 5104$ & 101,900 & 3,856 & Deliver Envelope D Batch 5 (2A7 Thind Batch) \\
\hline & & & C. 103 & AN.103 & 5,2804 & $8 / 1104$ & 290,500 & 10,424 & \\
\hline & & & REIURN 2 & $A P-107$ & $8 / 11104$ & 811104 & 8,678 & 3311 & \\
\hline & & & $2 \mathrm{~N} 1.87$ & SY $=102$ & $9 / 17 / 04$ & $9 / 1304$ & 3,000 & Q) & \\
\hline & & & SPN87 & $5 Y-102$ & $9 / 17 / 04$ & $9 / 1704$ & 25,0001 & 0 & \\
\hline & & & 1FL95 & AW-105 & $9 / 18 / 04$ & $9[1904$ & 200,000 & 0. & \\
\hline & & & WATER & $s y-102$ & $9 / 17 / 04$ & $9 / 1904$ & 426,000 & 0 & \\
\hline & & & WATER & AW-105 & $9 / 19 / 04$ & $9 / 19 / 04$ & 8,000 & of & \\
\hline & & & WATER & $S Y-102$ & $9 / 19 / 04$ & 9/19na & 6,000 & 0 & \\
\hline & & & EVAPF & $\mathrm{AW}+102$ & $9 / 20,04$ & 920004 & $85 \mathrm{ma}$ & of & \\
\hline & & & RETURN I & AP 107 & $9 / 2704$ & $9 / 2704$ & 8,681 & 331 & \\
\hline & & & $\mathrm{AW}-102$ & $2+2-A$ & $9 / 26 / 04$ & $9 / 30 / 04$ & 802,100 & 29 & \\
\hline & & & $262-1$ & $\mathrm{AW}-105$ & $9 / 2604$ & $9 / 30104$ & 142,600 & 29 & \\
\hline & & & $242 \cdot A$ & LERF & 925.04 & $9 / 30 / 04$ & 659,500 & 0 & \\
\hline & & & AN-105 & AW-102 & 10204 & $50 / 5 / 44$ & 633.000 & 11,046 & \\
\hline & & & WESF & AN. 105 & $10 / 604$ & $10 / 6 / 04$ & 5,000 & of & \\
\hline & & & TNS8 & $A N^{\prime}-10 s$ & $10 / 6 / 04$ & $10 / 6 / 04$ & 750 & 250 & \\
\hline & & & TALB8 & AN-105 & $10 / 6,04$ & $10 / 6,04$ & 24,000 & 0 & \\
\hline & & & WATER & AN-10S & $10 / 6,04$ & $10 / 6 / 04$ & 35.000 & 0 & \\
\hline & & & WATER & $A N-105$ & 10150 & $10 / 504$ & $27, \infty 00$ & a & \\
\hline & & & $34 L 87$ & AN-105 & $10 / 6104$ & $10 / 6 / 04$ & 50,000 & 0 & \\
\hline & & & EVAPF & AN-10S & $10 / 6 / 04$ & $10 / 6,04$ & 120,000 & 0 & \\
\hline & & & $A W-105$ & AN-10S & $10 / 5104$ & $10 / 104$ & 235,200 & 0 & \\
\hline & & & AW-10S & $\mathrm{AW}-105$ & $10 / 7 \times 4$ & 107704 & 56,917 & of & \\
\hline & & & RETURN 2 & AP. 107 & 10004 & $100 / 04$ & 8.678 & 33] & \\
\hline & & & EETURN 1 & AP-107 & $11 / 2304$ & $11 / 23 / 04$ & 8,681 & 331 & \\
\hline & & & $A-102$ & AN-103 & $8 / 1104$ & $11 / 30 / 04$ & 102,200 & 744 & \\
\hline & & & EETURN 2 & AP: 107 & $12 / 304$ & $12 / 3 \times 4$ & 8,678 & 331 & \\
\hline & & & AN-103 & $\Delta N-104$ & $1 / 16,05$ & $1 / 16,05$ & 3,461 & 91 & \\
\hline & & & AN-103 & $A W-104$ & $1 / 16005$ & I/16RSS & 17,750 & 468 & \\
\hline & & & $A N-103$ & AW. 103 & $1 / 16005$ & $1 / 1605$ & 3,897 & 103 & \\
\hline & & & $A N-I 03$ & AP-10S & $1 / 16,05$ & $1 / 1205$ & 43,576 & 1,147 & \\
\hline & & & $4 N-103$ & AP. 103 & $1 / 17 / 05$ & $1 / 1705$ & 42,878 & 1,128 & \\
\hline & & & AN.103 & AN. 101 & $1 / 1705$ & $1 / 170 s$ & 28,978 & 762 & \\
\hline & & & $A N-103$ & $\mid A P-101$ & $1 / 17 / 05$ & $1 / 17 / 5$ & 38,554 & 1.014 & \\
\hline & & & RENURN I & AP. 107 & $1 / 19,05$ & 1/19Nos & 8,681 & 331 & \\
\hline
\end{tabular}


HNF-SD-TWR-AGA-003

Revision 0

Table D-1. Pretreatment Processing Aiternative 1 -

Projected Transfers Through April 2011 (10 sheets).

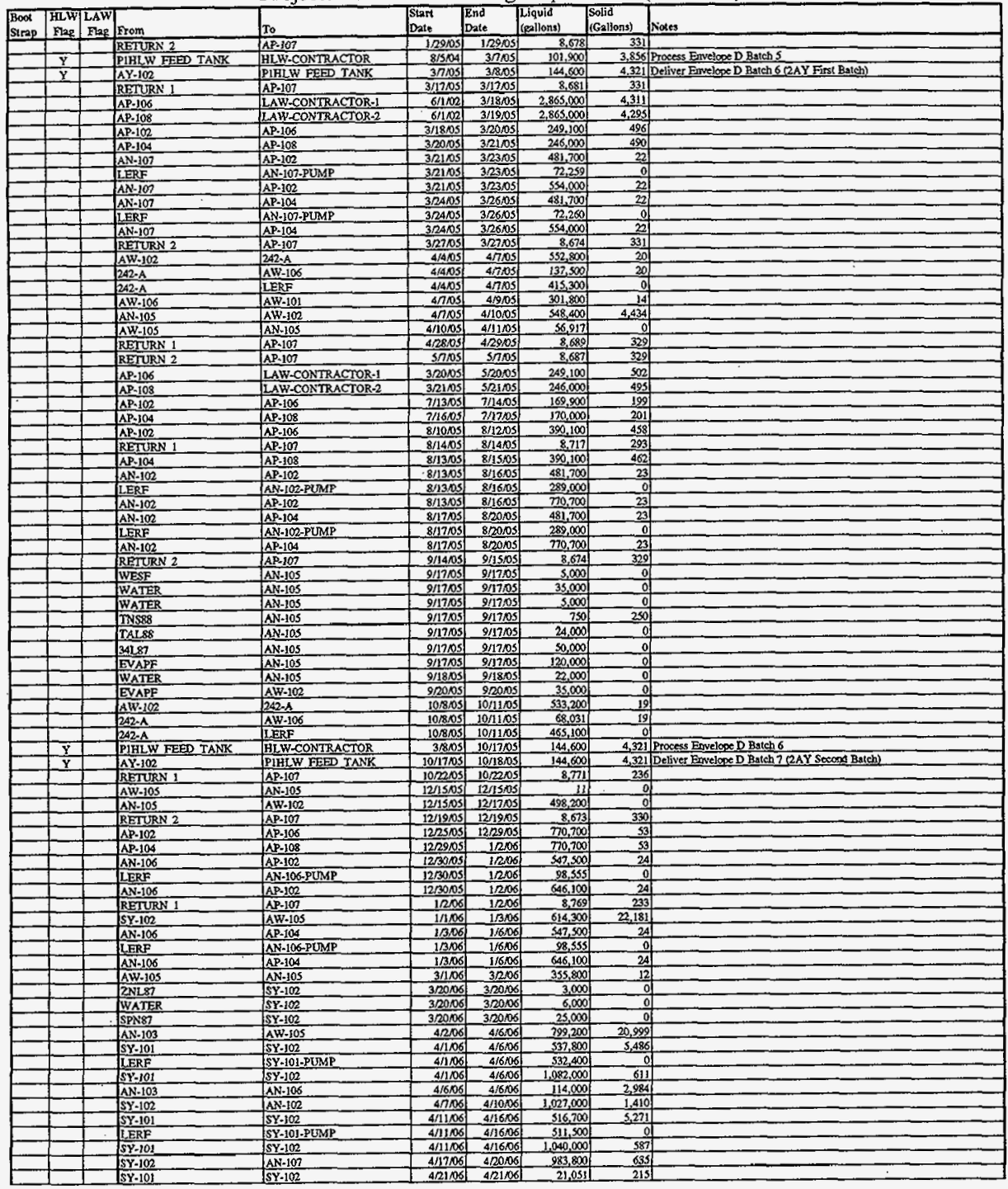


HNF-SD-TWR-AGA-003

Revision 0

Table D-1. Pretreatment Processing Alternative 1 -

Projected Transfers Through April 2011 (10 sheets).

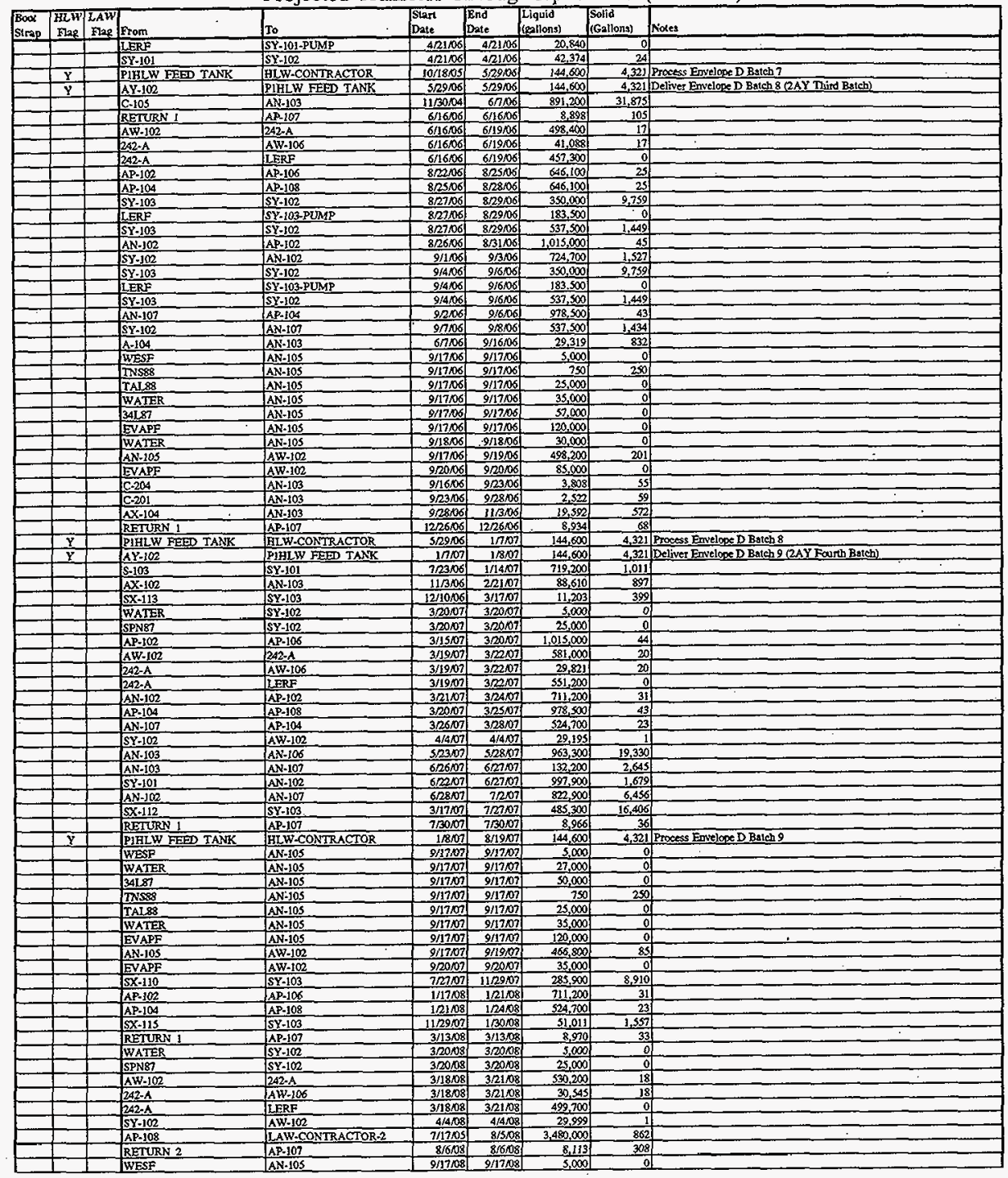

D-12 
HNF-SD-TWR-AGA-003

Revision 0

Table D-1. Pretreatment Processing Alternative 1 -

Projected Transfers Through April 2011 (10 sheets).

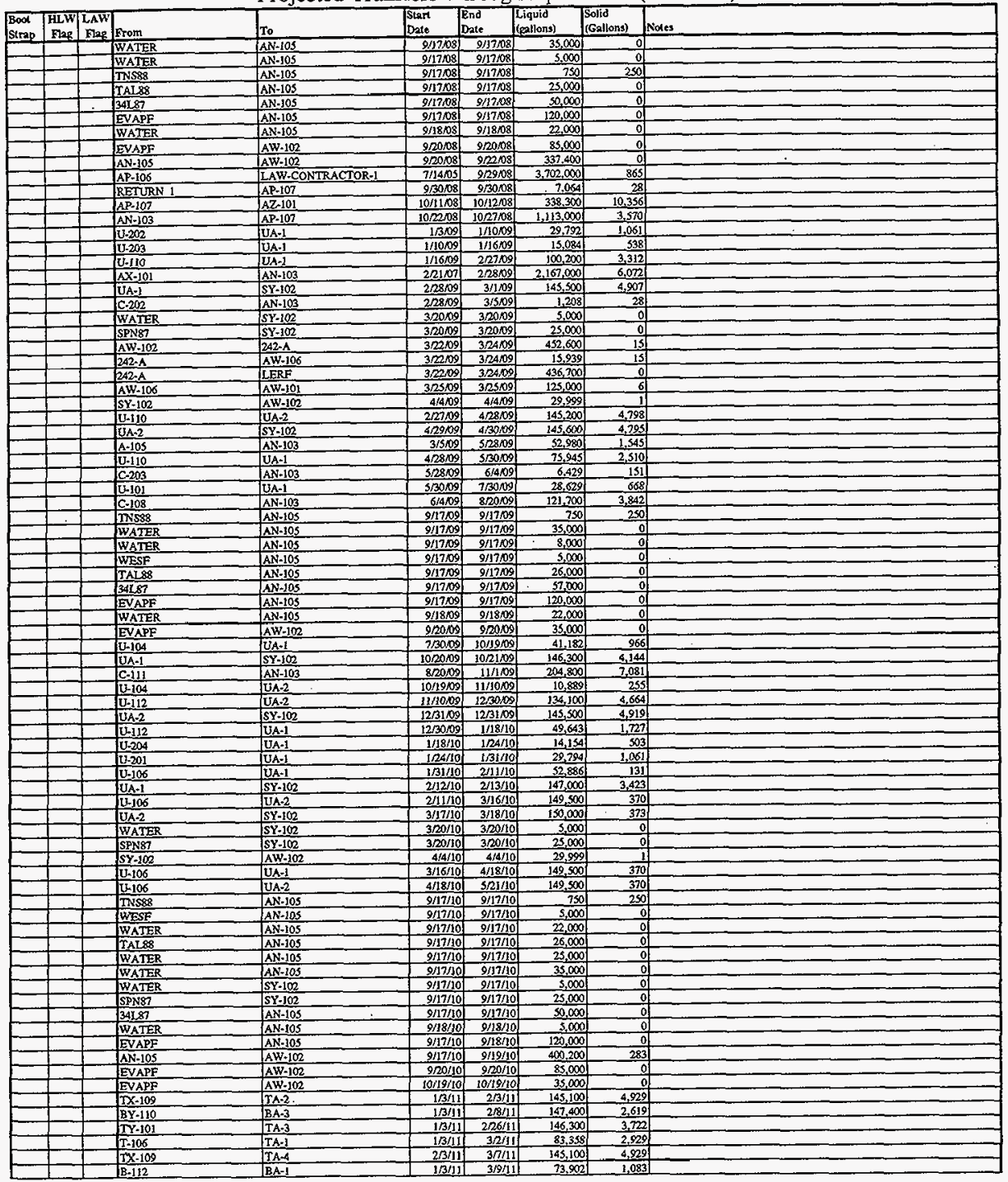


HNF-SD-TWR-AGA-003

Revision 0

Table D-1. Pretreatment Processing Alternative 1 -

Projected Transfers Through April 2011 (10 sheets).

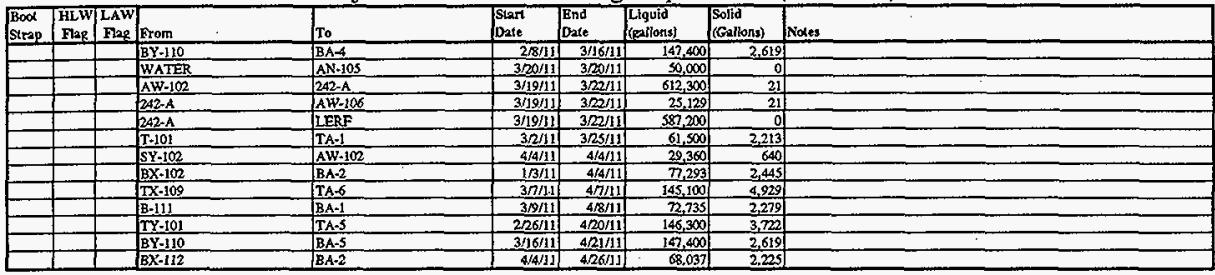

\section{D-14}




\section{APPENDIX E}

\section{SCHEDULES OF WASTE TRANSFERS FOR THE PHASE I HIGH-LEVEL WASTE PRETREATMENT PROCESSING ALTERNATIVES}


HNF-SD-TWR-AGA-003

Revision 0

This page intentionally left blank.

$+$

E-2 
HNF-SD-TWR-AGA-003

Revision 0

\section{APPENDIX E}

\section{SCHEDULES OF WASTE TRANSFERS FOR THE PHASE I HIGH-LEVEL WASTE PRETREATMENT PROCESSING ALTERNATIVES}

This appendix contains the schedules for Alternatives 1 and 2 to be used for purposes of comparing the two extremes: enhanced sludge washing all 3 tanks versus sludge washing all 3 tanks. These schedules are used as input to the Hanford Tank Waste Operation Simulator (HTWOS) model. 
Table E-1. Schedule for High-Level Waste Pretreatment Alternative 1.

(Sheet 1 of 3)

\begin{tabular}{|c|c|c|c|}
\hline Activity Description & $\begin{array}{c}\text { Transfer } \\
\text { Volume (L) }\end{array}$ & Late Start Date & $\begin{array}{c}\text { Duration } \\
\text { (days) }\end{array}$ \\
\hline \multicolumn{4}{|c|}{ "Bootstrap" Transfer Supporting 241-AZ-101 Pretreatment } \\
\hline 1. Transfer AY-101 Supernatant to AP-108 & $3,200,000$ & $6 / 1 / 00$ & 30 \\
\hline \multicolumn{4}{|c|}{ Other Operations Supporting $241-\mathrm{AZ}-101$ Pretreatment } \\
\hline ** Perform 241-AZ-101 Mixer Pump Test & (x) & $4 / 21 / 98$ & 30 \\
\hline ** Obtain Grab Samples of Sludge from 241-AZ-101 for PHMC & (x) & $5 / 21 / 98$ & 14 \\
\hline 2a. Complete 241-AZ-101 ln-Tank Evaporation to $5 \mathrm{M} \mathrm{Na}$ & 0 & $7 / 1 / 00$ & 0 \\
\hline \multicolumn{4}{|c|}{ 241-AZ-101 Sludge Washing Operations } \\
\hline 4a. Add 50 wt\% Caustic Solution to 241-AZ-101 and Mix Siudge & 70,000 & $7 / 1 / 00$ & 30 \\
\hline 5a. Wait for Sludge to Settle in 241-AZ-101 & 暗 & $7 / 31 / 00$ & 30 \\
\hline $\begin{array}{l}\text { 6a. Sample Sludge and Decant 241-AZ-101 Supernatant to } \\
\text { 241-AY-101 }\end{array}$ & $2,700,000$ & $8 / 30 / 00$ & 30 \\
\hline 4b. Add Wash Solution to 241-AZ-101 and Mix Sludge & 550,000 & $9 / 29 / 00$ & 30 \\
\hline 5b. Wait for Sludge to Settle in $241-A Z-101$ & rax & $10 / 29 / 00$ & 30 \\
\hline $\begin{array}{l}\text { 6b. Sample Sludge and Decant 241-AZ-101 Supernatant to AW- } \\
105\end{array}$ & 510,000 & $11 / 28 / 00$ & 30 \\
\hline 4c. Add Wash Solution to 241-AZ-101 and Mix Sludge & 550,000 & $12 / 28 / 00$ & 30 \\
\hline 5c. Wait for Sludge to Settle in 241-AZ-101 & & $1 / 27 / 01$ & 30 \\
\hline $\begin{array}{l}\text { 6c. Sample Siudge and Decant 241-AZ-101 Supernatant to AW- } \\
105\end{array}$ & 530,000 & $2 / 26 / 01$ & 30 \\
\hline 4d. Add Wash Solution to 241-AZ-101 and Mix Sludge & 550,000 & $3 / 28 / 01$ & 30 \\
\hline 5d. Wait for Sludge to Settle in 241-AZ-101 & & $4 / 27 / 01$ & 30 \\
\hline $\begin{array}{l}\text { 6d. Sample Sludge and Decant 241-AZ-101 Supernatant to AW- } \\
105\end{array}$ & 550,000 & $5 / 27 / 01$ & 30 \\
\hline \multicolumn{4}{|c|}{ 241-AZ-101 Feed Staging Operations } \\
\hline 7. Add Transfer Solution to 241-AZ-101 & 310,000 & $6 / 26 / 01$ & 10 \\
\hline ** Mix/Sample/Evaluate 241-AZ-101 Sludge & & $7 / 6 / 01$ & 70 \\
\hline $\begin{array}{l}\text { ** Take Representative Samples from 241-AZ-101, Provide Them } \\
\text { to the Private Contractor, and Wait for Waste Form Qualification }\end{array}$ & & $9 / 14 / 01$ & 245 \\
\hline 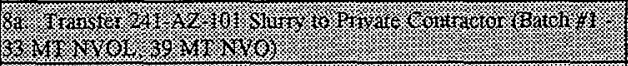 & & (3) & 经 \\
\hline W ( & & 6 (6) & 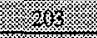 \\
\hline 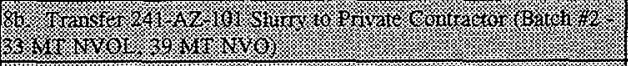 & & & $\sqrt{2}$ \\
\hline 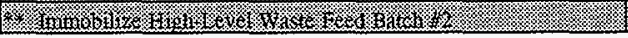 & 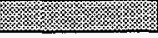 & (3.). & (6) \\
\hline
\end{tabular}


HNF-SD-TWR-AGA-003

Revision 0

Table E-1. Schedule for High-Level Waste Pretreatment Alternative 1. (Sheet 2 of 3)

\begin{tabular}{|c|c|c|c|}
\hline Activity Description & $\begin{array}{c}\text { Transfer } \\
\text { Volume (L) }\end{array}$ & Late Start Date & $\begin{array}{c}\text { Duration } \\
\text { (days) }\end{array}$ \\
\hline \multicolumn{4}{|c|}{ Other Operations Supporting 241-AZ-102 Pretreatment } \\
\hline ** Obtain Core Samples from 241-AZ-102 for PHMC & (3.1.6. & TBD & 150 \\
\hline 2b. Complete 241-AZ-102 In-Tank Evaporation to $5 \mathrm{M}$ Na & $1,700,000$ & $11 / 8 / 01$ & 0 \\
\hline \multicolumn{4}{|c|}{ 241-AZ-102 Shdge Washing Operations } \\
\hline $\begin{array}{l}\text { 10a. Add } 50 \text { wt \% Caustic Solution to 241-AZ-102 and Mix } \\
\text { Sludge }\end{array}$ & 120,000 & $11 / 8 / 01$ & 30 \\
\hline 11a. Wait for Sludge to Settle in 241-AZ-102 & & $12 / 8 / 01$ & 30 \\
\hline $\begin{array}{l}\text { 12a. Sample Sludge and Decant 241-AZ-102 Supernatant to AY- } \\
\text { 101/241-AN-105 }\end{array}$ & $1,200,000$ & $1 / 7 / 02$ & 30 \\
\hline 10b. Add Wash Solution to 241-AZ-102 and Mix Sludge & $2,400,000$ & $2 / 6 / 02$ & 30 \\
\hline 11b. Wait for Sludge to Settle in 241-AZ-102 & & $3 / 8 / 02$ & 30 \\
\hline $\begin{array}{l}\text { 12b. Sample Sludge and Decant 241-AZ-102 Supernatant to } \\
\text { 241-AN-105 }\end{array}$ & $2,300,000$ & $4 / 7 / 02$ & 30 \\
\hline 10c. Add Wash Solution to 241-AZ-102 and Mix Sludge & $2,400,000$ & $5 / 7 / 02$ & 30 \\
\hline 11c. Wait for Sludge to Settle in 241-AZ-102 & ( & $6 / 6 / 02$ & 30 \\
\hline $\begin{array}{l}\text { 12c. Sample Sludge and Decant 241-AZ-102 Supernatant to } \\
\text { 241-AN-105 }\end{array}$ & $2,400,000$ & $7 / 6 / 02$ & 30 \\
\hline \multicolumn{4}{|c|}{ 241-AZ-102 Feed Staging Operations } \\
\hline 13. Add Transfer Solution to 241-AZ-102 & 580,000 & $8 / 5 / 02$ & 10 \\
\hline ** Mix/Sample/Evaluate 241-AZ-102 Sludge & & $8 / 15 / 02$ & 70 \\
\hline $\begin{array}{l}\text { ** Take Representative Samples from 241-AZ-102, Provide Them } \\
\text { to the Private Contractor, and Wait for Waste Form Qualification }\end{array}$ & & $10 / 24 / 02$ & 245 \\
\hline 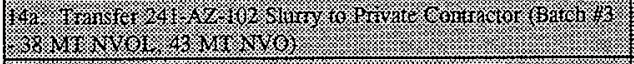 & & 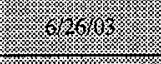 & \\
\hline 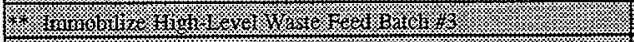 & & 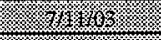 & 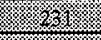 \\
\hline 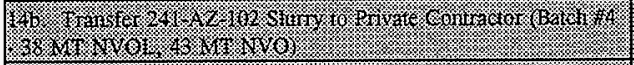 & 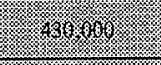 & (3) & 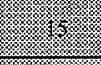 \\
\hline 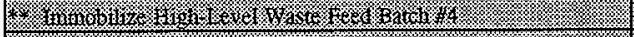 & & 26 & / \\
\hline 4. & & 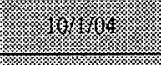 & 8 \\
\hline 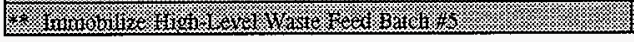 & 2 & 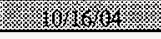 & 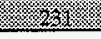 \\
\hline
\end{tabular}


HNF-SD-TWR-AGA-003

Revision 0

Table E-1. Schedule for High-Level Waste Pretreatment Alternative 1.

(Sheet 3 of 3)

\begin{tabular}{|c|c|c|c|}
\hline Activity Description & $\begin{array}{c}\text { Transfer } \\
\text { Volume (L) }\end{array}$ & Late Start Date & $\begin{array}{l}\text { Duration } \\
\text { (days) }\end{array}$ \\
\hline \multicolumn{4}{|c|}{ Other Operations Supporting 241-AY-102 Pretreatment } \\
\hline ** Obtain Core Samples from 241-AY-102 for PHMC & ל\%: & TBD & 150 \\
\hline \multicolumn{4}{|c|}{ 241-AY-102 Sludge Washing Operations } \\
\hline $\begin{array}{l}\text { 16a. Add } 50 \text { wt \% Caustic Solution to 241-AY-102 and Mix } \\
\text { Sludge }\end{array}$ & 200,000 & $10 / 3 / 03$ & 30 \\
\hline 17a. Wait for Sludge to Settle in $241-\mathrm{AY}-102$ & (1) & $11 / 2 / 03$ & 30 \\
\hline $\begin{array}{l}\text { 18a. Sample Sludge and Decant 241-AY-102 Supernatant to } \\
\text { 241-AN-105 }\end{array}$ & $2,600,000$ & $12 / 2 / 03$ & 30 \\
\hline 16b. Add Wash Solution to 241-AY-102 and Mix Sludge & $1,200,000$ & $1 / 1 / 04$ & 30 \\
\hline 17b. Wait for Sludge to Settle in 241-AY-102 & 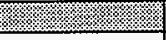 & $1 / 31 / 04$ & 30 \\
\hline $\begin{array}{l}\text { 18b. Sample Sludge and Decant 241-AY-102 Supernatant to } \\
\text { 241-AN-105 }\end{array}$ & $1,200,000$ & $3 / 1 / 04$ & 30 \\
\hline 16c. Add Wash Solution to 241-AY-102 and Mix Sludge & $1,200,000$ & $3 / 31 / 04$ & 30 \\
\hline 17c. Wait for Sludge to Settle in $241-\mathrm{AY}-102$ & & $4 / 30 / 04$ & 30 \\
\hline $\begin{array}{l}\text { 18c. Sample Sludge and Decant 241-AY-102 Supernatant to } \\
\text { 241-AN-105 }\end{array}$ & $1,200,000$ & $5 / 30 / 04$ & 30 \\
\hline \multicolumn{4}{|c|}{ 241-AY-102 Feed Staging Operations } \\
\hline 19. Add Transfer Solution to 241-AY-102 & $1,300,000$ & $6 / 29 / 04$ & 10 \\
\hline ** Mix/Sample/Evaluate 241-AY-102 Sludge & & $7 / 9 / 04$ & 70 \\
\hline $\begin{array}{l}\text { ** Take Representative Samples from 241-AY-102, Provide Them } \\
\text { to the Private Contractor, and Wait for Waste Form Qualification }\end{array}$ & & $9 / 17 / 04$ & 245 \\
\hline 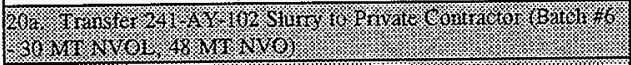 & & 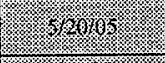 & \\
\hline \% Winind & Wי & 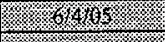 & 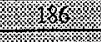 \\
\hline 261. & & 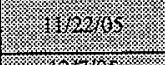 & S \\
\hline Y. & s & 篗 & 管籍 \\
\hline 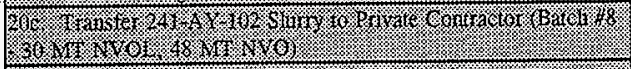 & 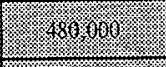 & (3. & 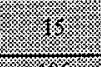 \\
\hline 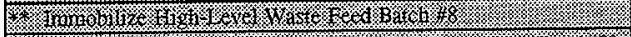 & & (3) & 8 \\
\hline 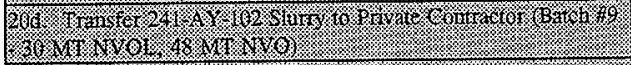 & . & (3) & 6) \\
\hline 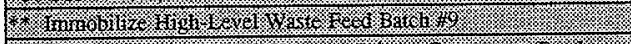 & & Y. & 86 \\
\hline 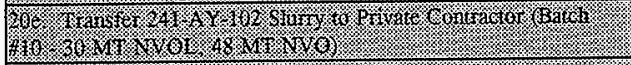 & 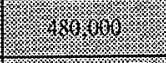 & & \\
\hline 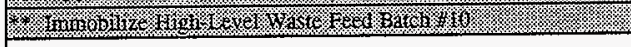 & & \% & 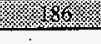 \\
\hline $\begin{array}{l}* * \text { Complete High-Level Waste Immobilization ( } 330 \mathrm{MT} \text { NVOL, } \\
450 \mathrm{MT} \text { NVO) }\end{array}$ & & $12 / 22 / 07$ & 0 \\
\hline
\end{tabular}

NVOL $=$ Non-Volatile Oxides Excluding Sodium and Silicon

NVO $=$ Non-Volatile Oxides

General Notes:

- The activity numbers correspond to the illustration in Figure 2-1.

- Activities with no numbers (**) are not represented in Figure 2-1.

- The waste transfers shown in this schedule are based on conservative maximum durations, and may not correspond to the modeled schedule provided in Appendix D. 
HNF-SD-TWR-AGA-003

Revision 0

Table E-2. Schedule for High-Level Waste Pretreatment Alternative 2.

(Sheet 1 of 3 )

\begin{tabular}{|c|c|c|c|}
\hline Activity Description & $\begin{array}{c}\text { Transfer } \\
\text { Volume (L) }\end{array}$ & Late Start Date & $\begin{array}{c}\text { Duration } \\
\text { (days) }\end{array}$ \\
\hline \multicolumn{4}{|c|}{ "Bootstrap" Transfer Supporting 241-AZ-101 Pretreatment } \\
\hline 1. Transfer AY-101 Supernatant to AP-108 & $3,200,000$ & $8 / 30 / 00$ & 30 \\
\hline \multicolumn{4}{|c|}{ Other Operations Supporting 241-AZ-101 Pretreatment } \\
\hline ** Perform 241-AZ-101 Mixer Pump Test & & $4 / 21 / 98$ & 30 \\
\hline ** Obtain Grab Samples of Sludge from 241-AZ-101 for PHMC & & $5 / 21 / 98$ & 14 \\
\hline 2a. Complete 241-AZ-101 In-Tank Evaporation to $5 M \mathrm{Na}$ & 0 & $8 / 30 / 00$ & 0 \\
\hline \multicolumn{4}{|c|}{ "Bootstrap" Transfer Supporting 241-AZ-101 Pretreatment } \\
\hline 3. Transfer 241-AZ-101 Supernatant to $A X-101$ & $2,400,000$ & $8 / 30 / 00$ & 30 \\
\hline \multicolumn{4}{|c|}{ 241-AZ-101 Sludge Washing Operations } \\
\hline 4a. Add Wash Solution to 241-AZ-101 and Mix Sludge & 550,000 & $9 / 29 / 00$ & 30 \\
\hline 5a. Wait for Siudge to Settle in 241-AZ-101 & & $10 / 29 / 00$ & 30 \\
\hline $\begin{array}{l}\text { 6a. Sample Sludge and Decant 241-AZ-101 Supernatant to AW- } \\
105\end{array}$ & 600,000 & $11 / 28 / 00$ & 30 \\
\hline 4b. Add Wash Solution to $241-\mathrm{AZ}-101$ and Mix Sludge & 550,000 & $12 / 28 / 00$ & 30 \\
\hline 5b. Wait for Sludge to Settle in 241-AZ-101 & & $1 / 27 / 01$ & 30 \\
\hline $\begin{array}{l}\text { 6b. Sample Sludge and Decant 241-AZ-101 Supernatant to AW- } \\
105\end{array}$ & 520,000 & $2 / 26 / 01$ & 30 \\
\hline 4c. Add Wash Solution to 241-AZ-101 and Mix Sludge & 550,000 & $3 / 28 / 01$ & 30 \\
\hline 5c. Wait for Sludge to Settle in 241-AZ-101 & & $4 / 27 / 01$ & 30 \\
\hline $\begin{array}{l}\text { 6c. Sample Sludge and Decant 241-AZ-101 Supematant to AW- } \\
105\end{array}$ & 540,000 & $5 / 27 / 01$ & 30 \\
\hline \multicolumn{4}{|c|}{ 241-AZ-101 Feed Staging Operations } \\
\hline 7. Add Transfer Solution to $241-\mathrm{AZ}-101$ & 550,000 & $6 / 26 / 01$ & 10 \\
\hline ** Mix/Sample/Evaluate 241-AZ-101 Sludge & & $7 / 6 / 01$ & 70 \\
\hline $\begin{array}{l}\text { ** Take Representative Samples from 241-AZ-101, Provide Them } \\
\text { to the Private Contractor, and Wait for Waste Form Qualification }\end{array}$ & & $9 / 14 / 01$ & 245 \\
\hline 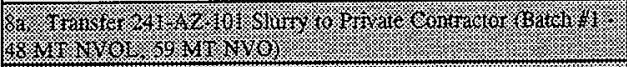 & 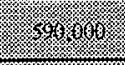 & 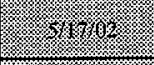 & x. \\
\hline 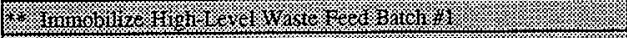 & & a. & (2) \\
\hline 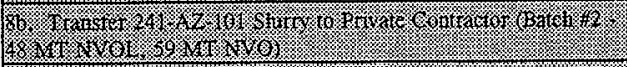 & 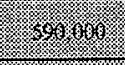 & \% & 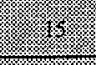 \\
\hline 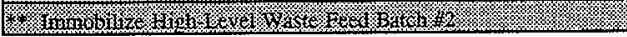 & & 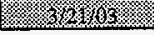 & 28 \\
\hline
\end{tabular}


Table E-2. Schedule for High-Level Waste Pretreatment Alternative 2.

(Sheet 2 of 3)

\begin{tabular}{|c|c|c|c|}
\hline Activity Description & $\begin{array}{c}\text { Transfer } \\
\text { Volume (L) }\end{array}$ & Late Start Date & $\begin{array}{c}\text { Duration } \\
\text { (days) }\end{array}$ \\
\hline \multicolumn{4}{|c|}{ Other Operations Supporting 241-AZ-102 Pretreatment } \\
\hline ** Obtain Core Samples from 241-AZ-102 for PHMC & \% & TBD & 150 \\
\hline 2b. Complete 241-AZ-102 In-Tank Evaporation to $5 \mathrm{M} \mathrm{Na}$ & $1,700,000$ & $1 / 8 / 02$ & 0 \\
\hline \multicolumn{4}{|c|}{ "Bootstrap" Transfer Supporting 241-AZ-102 Pretreatment } \\
\hline 9. Transfer 241-AZ-102 Supernatant to AY-101 and AN-104 & $1,300,000$ & $1 / 8 / 02$ & 30 \\
\hline \multicolumn{4}{|c|}{ 241-AZ-102 Sludge Washing Operations } \\
\hline 10a. Add Wash Solution to 241-AZ-102 and Mix Sludge & 810,000 & $2 / 7 / 02$ & 30 \\
\hline 11a. Wait for Sludge to Settle in 241-AZ-102 & (3. & $3 / 9 / 02$ & 30 \\
\hline $\begin{array}{l}\text { 12a. Sample Sludge and Decant 241-AZ-102 Supernatant to } \\
241-\mathrm{AN}-105\end{array}$ & 250,000 & $4 / 8 / 02$ & 30 \\
\hline 10b. Add Wash Solution to 241-AZ-102 and Mix Sludge & 810,000 & $5 / 8 / 02$ & 30 \\
\hline 11b. Wait for Sludge to Settle in 241-AZ-102 & 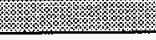 & $6 / 7 / 02$ & 30 \\
\hline $\begin{array}{l}\text { 12b. Sample Sludge and Decant 241-AZ-102 Supernatant to } \\
\text { 241-AN-105 }\end{array}$ & 780,000 & $7 / 7 / 02$ & 30 \\
\hline 10c. Add Wash Solution to 241-AZ-102 and Mix Sludge & 810,000 & $8 / 6 / 02$ & 30 \\
\hline 11c. Wait for Sludge to Settle in 241-AZ-102 & (1) & $9 / 5 / 02$ & 30 \\
\hline $\begin{array}{l}\text { 12c. Sample Sludge and Decant 241-AZ-102 Supernatant to } \\
\text { 241-AN-105 }\end{array}$ & 790,000 & $10 / 5 / 02$ & 30 \\
\hline 10d. Add Wash Solution to 241-AZ-102 and Mix Sludge & 810,000 & $11 / 4 / 02$ & 30 \\
\hline 11d. Wait for Sludge to Settle in 241-AZ-102 & 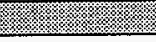 & $12 / 4 / 02$ & 30 \\
\hline $\begin{array}{l}\text { 12d. Sample Sludge and Decant 241-AZ-102 Supematant to } \\
\text { 241-AN-105 }\end{array}$ & 800,000 & $1 / 3 / 03$ & 30 \\
\hline \multicolumn{4}{|c|}{ 241-AZ-102 Feed Staging Operations } \\
\hline 13. Add Transfer Solution to $241-\mathrm{AZ}-102$ & 820,000 & $2 / 2 / 03$ & 10. \\
\hline ** Mix/Sample/Evaluate 241-AZ-102 Sludge & & $2 / 12 / 03$ & 70 \\
\hline $\begin{array}{l}\text { ** Take Representative Samples from 241-AZ-102, Provide Them } \\
\text { to the Private Contractor, and Wait for Waste Form Qualification }\end{array}$ & & $4 / 23 / 03$ & 245 \\
\hline 4. & 46 & 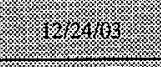 & \\
\hline 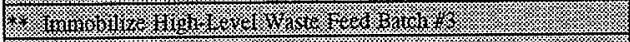 & & 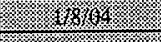 & 3. \\
\hline H. & (3. & 86704 & \\
\hline 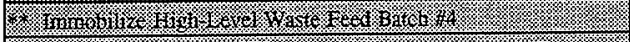 & & 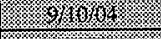 & 26 \\
\hline 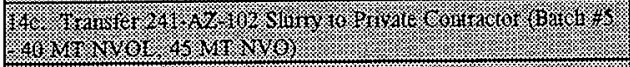 & 300 & & $x$ \\
\hline 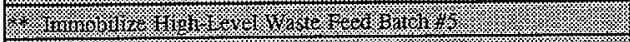 & & 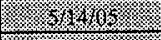 & 24.8 \\
\hline 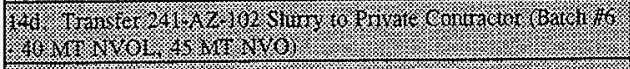 & 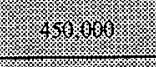 & (1) & 伭 \\
\hline 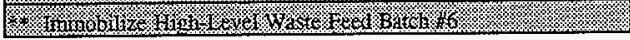 & & 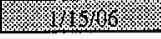 & 24 \\
\hline
\end{tabular}




\section{Revision 0}

Table E-2. Schedule for High-Level Waste Pretreatment Alternative 2. (Sheet 3 of 3)

\begin{tabular}{|c|c|c|c|}
\hline Activity Description & $\begin{array}{c}\text { Transfer } \\
\text { Volume (L) }\end{array}$ & Late Start Date & $\begin{array}{c}\text { Duration } \\
\text { (days) }\end{array}$ \\
\hline \multicolumn{4}{|c|}{ Other Operations Supporting 241-AY-102 Pretreatment } \\
\hline ** Obtain Core Samples from 241-AY-102 for PHMC & & TBD & 150 \\
\hline \multicolumn{4}{|c|}{ 241-AY-102 Sludge Washing Operations } \\
\hline $\begin{array}{l}\text { 15. Sample Sludge and Decant 241-AY-102 Supernatant to } \\
\text { 241-AN-105 }\end{array}$ & $2,000,000$ & $3 / 16 / 05$ & 30 \\
\hline 16a. Add Wash Solution to 241-AY-102 and Mix Shudge & $1,200,000$ & $4 / 15 / 05$ & 30 \\
\hline 17a. Wait for Sludge to Settle in 241-AY-102 & & $5 / 15 / 05$ & 30 \\
\hline $\begin{array}{l}\text { 18a. Sample Sludge and Decant 241-AY-102 Supernatant to } \\
241-\mathrm{AN}-105\end{array}$ & $1,400,000$ & $6 / 14 / 05$ & 30 \\
\hline 16b. Add Wash Solution to 241-AY-102 and Mix Shudge & $1,200,000$ & $7 / 14 / 05$ & 30 \\
\hline 17b. Wait for Sludge to Settle in $241-\mathrm{AY}-102$ & & $8 / 13 / 05$ & 30 \\
\hline $\begin{array}{l}\text { 18b. Sample Sludge and Decant 241-AY-102 Supernatant to } \\
241-\mathrm{AN}-105\end{array}$ & $1,200,000$ & $9 / 12 / 05$ & 30 \\
\hline \multicolumn{4}{|c|}{ 241-AY-102 Feed Staging Operations } \\
\hline 19. Add Transfer Solution to 241-AY-102 & $1,600,000$ & $10 / 12 / 05$ & 10 \\
\hline ** Mix/Sample/Evaluate 241-AY-102 Sludge & & $10 / 22 / 05$ & 70 \\
\hline $\begin{array}{l}\text { ** Take Representative Samples from 241-AY-102, Provide Them } \\
\text { to the Private Contractor, and Wait for Waste Form Qualification }\end{array}$ & & $12 / 31 / 05$ & 245 \\
\hline 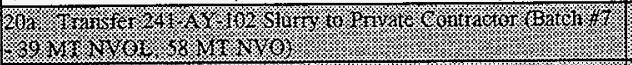 & 88 & 被 & \\
\hline 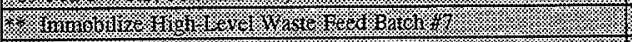 & 5 & (2) $17 \%$ & 230 \\
\hline 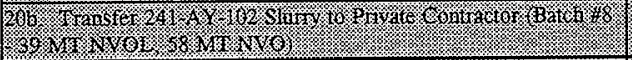 & 36. & $4 \times 280$ & \\
\hline 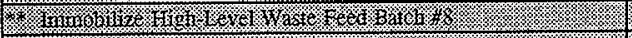 & & 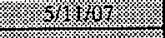 & (2.6. \\
\hline 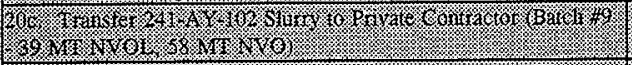 & (4) & 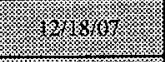 & 3. \\
\hline 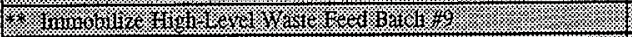 & & 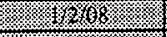 & 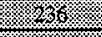 \\
\hline 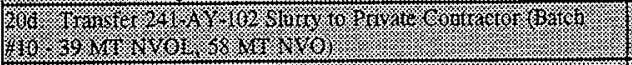 & $8 \times$ & wark & 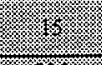 \\
\hline W & & 6 & 25 \\
\hline 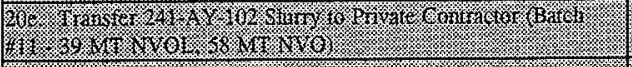 & 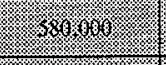 & & \\
\hline 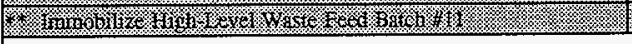 & & $48.8180 \%$ & 286 \\
\hline $\begin{array}{l}\text { ** Complete High-Level Waste Immobilization ( } 450 \mathrm{MT} \text { NVOL, } \\
590 \mathrm{MT} \text { NVO) }\end{array}$ & & $12 / 10 / 09$ & 0 \\
\hline
\end{tabular}

NVOL $=$ Non-Volatile Oxides Excluding Sodium and Silicon

NVO $=$ Non-Volatile Oxides

General Notes:

- The activity numbers correspond to the illustration in Figure 2-1.

- Activities with no numbers $(* *)$ are not represented in Figure 2-1.

- The waste transfers shown in this schedule are based on conservative maximum durations, and may not correspond to the modeled scheduie provided in Appendix D. 
HNF-SD-TWR-AGA-003

Revision 0

This page intentionally left blank. 


\section{APPENDIX F}

\section{PRELIMINARY EVALUATION OF PRETREATMENT ALTERNATIVES \\ FOR 241-AY-101, 241-C-101, \\ 241-C-102, 241-C-103, \\ 241-C-104, AND 241-C-105}


HNF-SD-TWR-AGA-003

Revision 0

This page intentionally left blank. 
HNF-SD-TWR-AGA-003

Revision 0

\begin{abstract}
APPENDIX F
PRELIMINARY EVALUATION OF PRETREATMENT ALTERNATTVES FOR 241-AY-101, 241-C-101, 241-C-102, 241-C-103, 241-C-104, AND 241-C-105
\end{abstract}

Six additional sources of HLW feed were identified in the Tank Waste Remediation System Operation and Utilization Plan (Kirkbride et al. 1997). The analysis was carried out based on sludge washing (without caustic leaching). This appendix presents the results of a preliminary evaluation of the 6 additional HLW feeds, based on wash and leach factors from Colton (1997), and the tank inventories from Kirkbride et al. (1997).

\title{
F1.0 SUMMARY
}

Tables F-1 through F-4 present the expected HLW feed compositions compared with the HLW feed specifications. The shaded cells denote concentrations which are outside of the Expanded Design Basis for Waste Envelope D (DOE 1996a and b).

\section{F1.1 ENHANCED SLUDGE WASHUNG RESULTS}

Table F-1 contains the estimated enhanced sludge washed compositions from 241-C-101, 241-C-102, and 241-C-103, and Table F-2 contains the estimated enhanced sludge washed compositions from 241-C-104, 241-C-105, and 241-AY-101. 
HNF-SD-TWR-AGA-003

Revision 0

Table F-1. Phase I High-Level Waste Envelope D Comparison for Selected Waste Constituents - Tanks 241-C-101, 241-C-102, and 241-C-103 (Based on $31 \mathrm{~g} / \mathrm{L}$ Non-Volatile Waste Oxides and Enhanced Sludge Washing).

\begin{tabular}{|c|c|c|c|c|c|}
\hline \multirow{2}{*}{ Component } & \multirow{2}{*}{$\begin{array}{l}\text { Minimum } \\
\text { concentration } \\
(\mathrm{g} / \mathrm{L})\end{array}$} & \multicolumn{3}{|c|}{$\begin{array}{l}\text { Estimated high-level waste feed concentrations } \\
\text { after enhanced sludge washing }(\mathrm{g} / \mathrm{L})\end{array}$} & \multirow{2}{*}{$\begin{array}{l}\text { Maximum } \\
\text { concentration } \\
\cdot(g / L)\end{array}$} \\
\hline & & $C-101$ & C-102 & C-103 & \\
\hline Aluminum & $1.30(0.33)$ & 2.46 & 5.14 & \% & $4.30(5.30)$ \\
\hline Bismuth & 0.00 & 0.00 & 0.00 & 0.00 & 0.86 \\
\hline Chlorine & 0.00 & 0.009 & 0.003 & 0.001 & 0.10 \\
\hline Chromium & 0.00 & 0.008 & 0.008 & 0.02 & $0.21(0.42)$ \\
\hline Fluorine & 0.00 & 0.00 & 0.16 & 0.00 & 1.10 \\
\hline Iron & $2.60(1.70)$ & 8.92 & 5.63 & 2.24 & $8.90(13.00)$ \\
\hline Manganese & 0.00 & 0.00 & 0.00 & 0.00 & 2.00 \\
\hline Nickel & $0.05(0.00)$ & 0.56 & 0.48 & 0.21 & $0.73(1.00)$ \\
\hline Pallađium & 0.00 & 0.00 & 0.00 & 0.00 & 0.04 \\
\hline Phosphorus & 0.00 & 0.03 & 0.008 & 0.008 & 0.54 \\
\hline Potassium & 0.00 & 0.00 & 0.00 & 0.00 & 0.41 \\
\hline Rhodium & 0.00 & 0.00 & 0.00 & 0.00 & 0.04 \\
\hline Ruthenium & 0.00 & 0.00 & 0.00 & 0.00 & 0.11 \\
\hline Silicon & 0.00 & 0.0001 & 0.007 & 2.01 & 5.80 \\
\hline Silver & 0.00 & $3.20 \mathrm{E}-10$ & $4.21 \mathrm{E}-20$ & 8.04E-20 & 0.17 \\
\hline Sodium & $2.30(1.00)$ & 장 & (19.8 & 2.61 & $6.00(9.20)$ \\
\hline Sulfur & 0.00 & 0.06 & 0.008 & 0.0004 & 0.20 \\
\hline Titanium & 0.00 & 0.00 & 0.00 & 0.00 & 0.40 \\
\hline Uranium & 0.00 & \% & \% & 0.68 & 4.20 \\
\hline Zirconium & 0.00 & 0.00 & 0.00 & 0.00 & 4.60 \\
\hline
\end{tabular}

$\mathrm{NE}=$ Not estimated

General Notes

- Values enclosed in parentheses are for the Expanded Design Basis for HLW Processing as defined in the Phase I TWRS Privatization contracts (DOE 1996a and DOE 1996b).

- Shaded rows indicate component concentrations which are close to or outside of the HLW feed specifications.

- Analytes not shown are within the HLW feed specifications and/or have no significant impact on the immobilized HLW product. 


\section{Revision 0}

Table F-2. Phase I High-Level Waste Envelope D Comparison for Selected Waste Constituents - Tanks 241-C-104, 241-C-105, and 241-AY-101 (Based on $31 \mathrm{~g} / \mathrm{L}$ Non-Volatile Waste Oxides and Enhanced Sludge Washing).

\begin{tabular}{|c|c|c|c|c|c|}
\hline \multirow{2}{*}{ Component } & \multirow{2}{*}{$\begin{array}{c}\text { Minimum } \\
\text { concentration } \\
(\mathrm{g} / \mathrm{L})\end{array}$} & \multicolumn{3}{|c|}{$\begin{array}{c}\text { Estimated high-level waste feed concentrations } \\
\text { after enhanced sludge washing }(\mathrm{g} / \mathrm{L})\end{array}$} & \multirow{2}{*}{$\begin{array}{c}\text { Maximum } \\
\text { concentration } \\
(\mathrm{g} / \mathrm{L})\end{array}$} \\
\hline & & C-104 & C-105 & AY-101 & \\
\hline Aluminum & $1.30(0.33)$ & 1.67 & 1.13 & 2.23 & $4.30(5.30)$ \\
\hline Bismuth & 0.00 & 0.00 & 0.00 & 0.00001 & 0.86 \\
\hline Chlorine & 0.00 & 0.009 & 0.005 & 0.02 & 0.10 \\
\hline Chromium & 0.00 & 0.03 & 0.02 & 0.12 & $0.21(0.42)$ \\
\hline Fluorine & 0.00 & 0.67 & 0.00 & 0.09 & 1.10 \\
\hline Iron & $2.60(1.70)$ & 7.38 & 8.13 & 4.80 & $8.90(13.00)$ \\
\hline Manganese & 0.00 & 0.00 & 0.00 & 0.79 & 2.00 \\
\hline Nickel & $0.05(0.00)$ & 0.33 & 0.78 & 0.07 & $0.73(1.00)$ \\
\hline Palladiun & 0.00 & 0.00 & 0.00 & 0.00 & 0.04 \\
\hline Phosphorus & 0.00 & 0.01 & 0.00 & 0.39 & 0.54 \\
\hline Potassium & 0.00 & 0.00 & 0.00 & 0.21 & 0.41 \\
\hline Rhodium & 0.00 & 0.00 & 0.00 & 0.00 & 0.04 \\
\hline Ruthenium & 0.00 & 0.00 & 0.00 & 0.00 & 0.11 \\
\hline Silicon & 0.00 & 1.35 & 0.04 & 0.24 & 5.80 \\
\hline Silver & 0.00 & $3.64 \mathrm{E}-20$ & $5.69 \mathrm{E}-09$ & 0.00 & 0.17 \\
\hline Sodium & $2.30(1.00)$ & 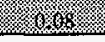 & (8) & 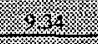 & $6.00(9.20)$ \\
\hline Sulfur & 0.00 & 0.004 & 0.02 & 8. & 0.20 \\
\hline Titanium & 0.00 & 0.00 & 0.00 & 0.00 & 0.40 \\
\hline Uranium & 0.00 & \% & 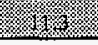 & 0.00005 & 4.20 \\
\hline Zirconium & 0.00 & 0.00 & 0.00 & 0.0001 & 4.60 \\
\hline
\end{tabular}

$\mathrm{NE}=$ Not estimated

General Notes

-Values enclosed in parentheses are for the Exparded Design Basis for HLW Processing as defined in the Phase I TWRS Privatization contracts (DOE 1996a and DOE 1996b).

-Shaded rows indicate component concentrations which are close to or outside of the HLW feed specifications.

-Analytes not shown are within the HLW feed specifications and/or have no significant impact on the immobilized HLW product. 
The results of this evaluation indicate that the uranium, aluminum, and sulfur specifications may be exceeded. After enhanced sludge washing, the uranium concentration will be above the maximum concentration of $4.20 \mathrm{~g} / \mathrm{L}$ (based on $31 \mathrm{~g} / \mathrm{L}$ non-volatile oxides $/ \mathrm{L}$ feed), for 241-C-101, 241-C-102, 241-C-104, and 241-C-105,. These sludges will require a significant degree of "chemical shimming" or blending with other sludges. Alternatively, if less of the aluminum is removed by enhanced sludge washing, the compositions could potentialiy stay within the specifications. (i.e., Tables F-3 and F-4 show that the uranium concentrations are below the maximum with no caustic leaching). Sludges with estimated sodium concentrations which fall below the minimum concentration are not considered problematic, since additional sodium can easily be added with the transfer solution.

For 241-C-103, enhanced sludge washing is not sufficient to remove enough aluminum to bring this feed within the HLW feed specifications. For 241-AY-101, the concentration of sulfur will be above the maximum. ${ }^{2}$

\section{F1.2 SLUDGE WASHING RESULTS}

For comparison, Tables F-3 and F-4 present the compositions of the 6 additional HLW feeds based on sludge washing (without caustic leaching).

${ }^{1}$ Chemical shimming is defined as the addition of inert chemicals to the HLW for purposes of diluting the concentration of chemical analytes below the maximum concentration specification. This adjustment would likely be performed with glass-forming materials, to minimize the impact on the immobilized HLW product volume.

${ }^{2}$ Preliminary evaluations using the Best-Basis Inventory estimates (Kupfer et al. 1997), indicate that the aluminum concentration in the $\mathrm{C}-103$ sludge and the sulfur concentration in the 241-AY-101 sludge may actually be lower, and that both of these feeds will be within the specifications for these constituents (Place et al. 1997). 
Table F-3. Phase I High-Level Waste Envelope D Comparison for Selected Waste Constituents - Tanks 241-C-101, 241-C-102, and 241-C-103 (Based on $31 \mathrm{~g} / \mathrm{L}$ Non-Volatile Waste Oxides and Sludge Washing).

\begin{tabular}{|c|c|c|c|c|c|}
\hline \multirow{2}{*}{ Component } & \multirow{2}{*}{$\begin{array}{c}\text { Minimum } \\
\text { concentration } \\
(\mathrm{g} / \mathrm{L})\end{array}$} & \multicolumn{3}{|c|}{$\begin{array}{c}\text { Estimated high-level waste feed concentrations } \\
\text { after sludge washing }(\mathrm{g} / \mathrm{L})\end{array}$} & \multirow{2}{*}{$\begin{array}{c}\text { Maximum } \\
\text { concentration } \\
(\mathrm{g} / \mathrm{L})\end{array}$} \\
\hline & & C-101 & C-102 & C-103 & \\
\hline Aluminum & $1.30(0.33)$ & 8.6 & ix. & (2) & $4.30(5.30)$ \\
\hline Bismuth & 0.00 & 0.00 & 0.00 & 0.00 & 0.86 \\
\hline Chiorine & 0.00 & 0.004 & 0.0009 & 0.0006 & 0.10 \\
\hline Chromium & 0.00 & 0.02 & 0.01 & 0.02 & $0.21(0.42)$ \\
\hline Fluorine & 0.00 & 0.00 & 0.05 & 0.00 & 1.10 \\
\hline Iron & $2.60(1.70)$ & 4.21 & (x) & 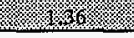 & $8.90(13.00)$ \\
\hline Manganese & 0.00 & 0.00 & 0.00 & 0.00 & 2.00 \\
\hline Nickel & $0.05(0.00)$ & 0.27 & 0.15 & 0.13 & $0.73(1.00)$ \\
\hline Palladium & 0.00 & 0.00 & 0.00 & 0.00 & 0.04 \\
\hline Phosphorus & 0.00 & 0.05 & 0.009 & 0.01 & 0.54 \\
\hline Potassium & 0.00 & 0.00 & 0.00 & 0.00 & 0.41 \\
\hline Rhodium & 0.00 & 0.00 & 0.00 & 0.00 & 0.04 \\
\hline Ruthenium & 0.00 & 0.00 & 0.00 & 0.00 & 0.11 \\
\hline Silicon & 0.00 & 0.00008 & 0.004 & 1.23 & 5.80 \\
\hline Silver & 0.00 & $1.50 \mathrm{E}-10$ & $1.27 \mathrm{E}-20$ & $4.90 \mathrm{E}-20$ & 0.17 \\
\hline Sodium & $2.30(1.00)$ & 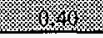 & (6) & 2.94 & $6.00(9.20)$ \\
\hline Sulfur & 0.00 & 0.03 & 0.003 & 0.0003 & 0.20 \\
\hline Titanium & 0.00 & 0.00 & 0.00 & 0.00 & 0.40 \\
\hline Uranium & 0.00 & 4.09 & 2.68 & 0.45 & 4.20 \\
\hline Zirconium & 0.00 & 0.00 & 0.00 & 0.00 & 4.60 \\
\hline
\end{tabular}

General Notes

-Values enclosed in parentheses are for the Expanded Design Basis for HLW Processing as defined in the Phase I TWRS Privatization contracts (DOE 1996a and DOE 1996b). specifications.

-Shaded rows indicate component concentrations which are close to or outside of the HLW feed

-Analytes not shown are within the HLW feed specifications and/or have no significant impact on the immobilized HLW product. 
Table F-4. Phase I High-Level Waste Envelope D Comparison for Selected Waste Constituents - Tanks 241-C-104, 241-C-105, and 241-AY-101 (Based on $31 \mathrm{~g} / \mathrm{L}$ Non-Volatile Waste Oxides and Sludge Washing).

\begin{tabular}{|c|c|c|c|c|c|}
\hline \multirow{2}{*}{ Component } & \multirow{2}{*}{$\begin{array}{c}\text { Minimum } \\
\text { concentration } \\
(\mathrm{g} / \mathrm{L})\end{array}$} & \multicolumn{3}{|c|}{$\begin{array}{l}\text { Estimated high-level waste feed concentrations } \\
\text { after sludge washing }(\mathrm{g} / \mathrm{L})\end{array}$} & \multirow{2}{*}{$\begin{array}{c}\text { Maximum } \\
\text { concentration } \\
(\mathrm{g} / \mathrm{L})\end{array}$} \\
\hline & & C-104 & C-105 & AY-101 & \\
\hline Aluminum & $1.30(0.33)$ & 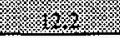 & $x_{1}$ & 2 & $4.30(5.30)$ \\
\hline Bismuth & 0.00 & 0.00 & 0.00 & 0.00 & 0.86 \\
\hline Chlorine & 0.00 & 0.002 & 0.0007 & 0.006 & 0.10 \\
\hline Chromium & 0.00 & 0.01 & 0.003 & 0.22 & $0.21(0.42)$ \\
\hline Fluorine & 0.00 & 0.15 & 0.00 & 0.04 & 1.10 \\
\hline Iron & $2.60(1.70)$ & 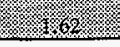 & 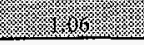 & 1.96 & $8.90(13.00)$ \\
\hline Manganese & 0.00 & 0.00 & 0.00 & 0.32 & 2.00 \\
\hline Nickel & $0.05(0.00)$ & 0.14 & 0.10 & 0.03 & $0.73(1.00)$ \\
\hline Palladium & 0.00 & 0.00 & 0.00 & 0.00 & 0.04 \\
\hline Phosphorus & 0.00 & 0.02 & 0.00 & . & 0.54 \\
\hline Potassium & 0.00 & 0.00 & 0.00 & 0.08 & 0.41 \\
\hline Rhodium & 0.00 & 0.00 & 0.00 & 0.00 & 0.04 \\
\hline Ruthenium & 0.00 & 0.00 & 0.00 & 0.00 & 0.11 \\
\hline Silicon & 0.00 & 0.53 & 0.02 & 0.15 & 5.80 \\
\hline Silver & 0.00 & $7.97 \mathrm{E}-21$ & $7.04 \mathrm{E}-10$ & 0.00 & 0.17 \\
\hline Sodium & $2.30(1.00)$ & 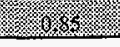 & (1) & 7.03 & $6.00(9.20)$ \\
\hline Sulfur & 0.00 & 0.0008 & 0.003 & 0.16 & 0.20 \\
\hline Titanium & 0.00 & 0.00 & 0.00 & 0.00 & 0.40 \\
\hline Uranium & 0.00 & 2.09 & 1.40 & 0.00002 & 4.20 \\
\hline Zirconium & 0.00 & 0.00 & 0.00 & 0.00005 & 4.60 \\
\hline
\end{tabular}

General Notes

-Values enclosed in parentheses are for the Expanded Design Basis for HLW Processing as defined in the Phase I TWRS Privatization contracts (DOE 1996a and DOE 1996b).

-Shaded rows indicate component concentrations which are close to or outside of the HLW feed specifications.

-Analytes not shown are within the HLW feed specifications and/or have no significant impact on the immobilized HLW product. 
HNF-SD-TWR-AGA-003

Revision 0

\section{F1.3 HIGH-LEVEL WASTE FEED QUANTITIES}

Table F-5 presents the quantities of feed from 241-C-101, 241-C-102, 241-C-103, 241-C-104, 241-C-105, and 241-AY-101.

Table F-5. High-Level Waste Feed Quantities (MT of Non-Volatile Oxides Excluding Sodium and Silicon).

\begin{tabular}{|l|c|c|c|c|c|c|}
\hline Tank & $241-\mathrm{C}-101$ & $241-\mathrm{C}-102$ & $241-\mathrm{C}-103$ & 241-C-104 & 241-C-105 & 241-AY-101 \\
\hline $\begin{array}{l}\text { Enhanced } \\
\text { Sludge } \\
\text { Washing }\end{array}$ & 51 & 251 & 65 & 110 & 45 & 24 \\
\hline $\begin{array}{l}\text { Sludge } \\
\text { Washing }\end{array}$ & 109 & 840 & 112 & 515 & 355 & 69 \\
\hline
\end{tabular}

Enhanced sludge washing significantly reduces the amount of feed material from these 6 tanks. If enhanced sludge washed, C-101, C-105, and AY-101 would be excluded from consideration, based on the "down" selection criteria used in Kirkbride et al. (1997). Tanks containing less than $60 \mathrm{MT}$ of HLW feed material were exluded. However, using the BestBasis Inventory estimates, only C-101 will not have enough material to meet the "down" selection criteria (Place et al. 1997). 
HNF-SD-TWR-AGA-003

Revision 0

F2.0 CONCLUSIONS

The uranium specification may need to be expanded to include the enhanced sludge washed compositions from 241-C-101, 241-C-102, 241-C-104, and 241-C-105. Other modifications to the feed specifications may be necessary for the aluminum in 241-C-103 and the sulfur in 241-AY-101, but a preliminary review using the best-basis inventory estimates indicate that these concentrations may be overestimated. If enhanced sludge washed, the estimated amount of material in 241-C-101 may not be sufficient to meet the "down" selection criteria for candidate HLW feeds.

Preliminary reviews, using the Best-Basis inventory estimates for these tanks, show different results. The 6 tanks identified are still valid choices, although the preferred pretreatment strategy (based on the ability to satisfy the HLW feed specifications) cannot be determined at this time. Further analysis is required to refine the composition and quantity estimates. These refinements will be used as input to the next revisions of this AGA and for the TWRS Operation and Utilization Plan. 
HNF-SD-TWR-AGA-003

Revision 0

\section{APPENDIX G}

\section{PHASE I HIGH-LEVEL WASTE PRETREATMENT AND FEED STAGING IMPLEMENTATION SUMMARY}


HNF-SD-TWR-AGA-003

Revision 0

This page intentionally left blank. 
HNF-SD-TWR-AGA-003

Revision 0

APPENDIX G

\section{PHASE I HIGH-LEVEL WASTE PRETREATMENT AND FEED STAGING IMPLEMENTATION SUMMARY}

This appendix summarizes the importance of on-going and planned Tank Farm projects in relation to Phase I high-level waste ( $\mathrm{HLW}$ ) pretreatment and feed staging. It is primarily focused on the HLW feed delivery needs, and it is acknowledged that other objectives may also influence these projects.

\section{G1.0 SUMMARY}

To satisfy the requirements of the Tank Waste Remediation System (TWRS) Privatization contracts (DOE 1996a and DOE 1996b), the HLW sludges in tanks 241-AZ-101, 241-AZ-102, and 241-AY-102/241-C-106 will require several operations prior to delivery to the Phase I Private Contractor. The following tank farm projects must be coordinated with the requirements for HLW feed delivery, some of which are indicated on the "Feed Delivery Mid-Level Logic for Phase I HLW Processing" (Boston 1997a). The key projects associated with Phase I HLW pretreatment and feed staging are as follows:

- Project W-151, "Tank 241-241-AZ-101 Waste Retrieval System"

- Project W-314, "Tank Farm Restoration and Safe Operations"

- Project W-320, "Tank 241-241-C-106 Waste Retrieval Sluicing System"

- Project W-211, "Initial Waste Retrieval System."

Examples of the types of pretreatment and feed staging operations that these projects must support include: chemical additions, solids mixing, transfer of supernatants out of the tank, sampling, heating, and single-shell tank (SST) and double-shell tank (DST) retrieval. Shortfalls in the scope of these projects will have a profound impact on the Project Hanford Management Contractor's (PHMC's) ability to deliver the HLW feeds meeting all contractual obligations. 
HNF-SD-TWR-AGA-003

Revision 0

\section{G2.0 SIGNIFICANCE OF TANK FARM PROJECTS TO THE HIGH-LEVEL WASTE FEED DELIVERY MISSION}

Each of the projects listed in Section G1.0 above contain activities. which are vital to Phase I HLW feed delivery. Decisions with regard to these projects must be made with respect to the contractual obligations for Privatization and cost and risk minimization considerations. This section briefly explains the significance of each.

\section{G2.1 PROJECT W-151, "TANK 241-AZ-101 WASTE RETRIEVAL SYSTEM"}

Project W-151 installs a retrieval system (two 300 -hp mixer pumps and ancillary equipment) into 241-AZ-101. This project is followed by operation of the mixer pumps to demonstrate the effectiveness of the selected retrieval system. Characteristics of the retrieval system such as, the effective cleaning radius (ECR), mixer pump jet forces on the in-tank components, sludge settling rates, and temperature data, will be measured.

Data obtained from the mixer pump test will be used to confirm assumptions regarding the PHMC's ability to retrieve and deliver the minimum order quantity of HLW feed from AZ-101, 241-AZ-102, and 241-AY-102/241-C-106. The data can also be used to predict the maximum quantity of sludge that can be retrieved if Phase I is extended beyond the minimum order quantity. The test results will be extrapolated to the other candidate HLW sludges for Phase I immobilization using available empirical models for DST retrieval or by developing new ones. The amount of sludge retrievable from each of the tanks needs to be well correlated with the physical properties of the sludge to ensure reliable predictions.

The ability to deliver the TWRS Privatization Phase IB contract specification for the minimum order quantity of $\mathrm{HLW}$ feed (Contract clause H.9) will depend on reliable predictions for DST sludge retrieval from 241-AZ-101, 241-AZ-102, and 241-AY-102/241-C-106. The penalty for failure to deliver the minimum order quantity will be negotiated during Phase IA, as the details regarding "Idle Facilities" payments are established (Contract clause H.30). Mixer pump test data will help to establish an appropriate margin for error in the specification. The absence of data from mixer pump testing prior to the U.S. Department of Energy's (DOE's) authorization to proceed with Phase IB will require a greater degree of conservatism in the Phase IB contract specification.

\section{G2.2 SLUDGE WASHING DEMONSTRATION}

A demonstration of in-tank processing (sludge washing) technology is currently not included in the scope of the identified projects. Such a demonstration will enable the PHMC to establish operational procedures, develop a sampling protocol, reduce the risks related to the ability to satisfy the contract feed specifications, and trouble-shoot the system/process, in advance of the need to deliver contract-compliant HLW feed. Since full-scale in-tank sludge washing has not been demonstrated on the Hanford Site, it is difficult to predict the impacts of these activities 
without such a demonstration. A demonstration of this technology will reduce the degree of conservatism that otherwise must be built into the specifications for the HLW feed(s).

\section{G2.3 PROJECT W-314, "TANK FARM RESTORATION AND SAFE OPERATIONS"}

The current Disposal Program planning baseline is to accelerate the transfer line and valve pit upgrades provided by Project W-314 to a date early enough to support the first waste transfers required to pretreat the HLW sludge. The reliability of the existing waste transfer lines is questionable, due to historical failures and potential solids settling and line plugging due to the combination of two- and three-inch diameter pipe in the existing transfer routings. Project W-314 is to replace the lines of questionable integrity and provide a continuous three-inch diameter transfer line system for all of the transfers in Figure 1-1 (Claghorn et al. 1997). Therefore, the decision was made (Boston 1997b) to replan W-314 based on an accelerated schedule.

Additional funds are required in Fiscal Years (FY) 1998 and 1999 to support the schedule acceleration of Project W-314. However, if these funds are not available (i.e, if the DOE elects to proceed with a higher risk path and defer the W-314 transfer line upgrades), the existing transfer lines can still be used. However, sufficient leàd time must exist to recover from a line failure. This alternative is not recommended, because recovery time is extremely difficult or impossible to predict. In anticipation of possible failures, the Phase IB contract may need to be written with a delayed startup date for HLW immobilization, to provide an adequate margin for recovery time.

A secondary benefit of deferring the line upgrades is that early pretreatment (i.e., a sludge washing demonstration) will reduce contractual risk and can provide valuable information for future project planning.

\section{G2.4 PROJECT W-320, "TANK 241-C-106 WASTE RETRIEVAL SLUICING SYSTEM"}

The sludge from 241-C-106 is necessary to satisfy the minimum order quantity requirements for HLW feed. Based on enhanced sludge washing the AZ Farm tanks and staging them as the first HLW sludge feeds, the sludge from $241-\mathrm{C}-106$ will provide up to 47.6 percent of the minimum order quantity, depending upon the amount of sludge retrieved from $241-\mathrm{AZ}-101$ and AZ-102. Processed alone (first), 241-C-106 can provide up to 80 percent of the minimum order quantity.

Table G2-1 presents the quantity of Waste Envelope D feed available based on the expected range of retrieval efficiencies, and caustic and water washing. 
HNF-SD-TWR-AGA-003

Revision 0

Table G2-1. Estimated Quantities of High-Level Waste Feed.

\begin{tabular}{|c|c|c|}
\hline & Minimum $^{2}$ & Maximum (100\% Retrieval) \\
\hline \multicolumn{3}{|c|}{ Water Washing } \\
\hline 241-AZ-101 & 86.8 & 96.4 \\
\hline 241-AZ-102 & 96.9 & 161.5 \\
\hline 241-AY-102/24I-C-106 & 169.8 & 193.8 \\
\hline 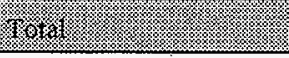 & 8.8 .8 & 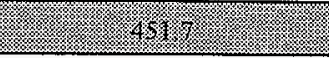 \\
\hline \multicolumn{3}{|c|}{ Caustic Washing } \\
\hline 241-AZ-101 & 59.9 & 66.6 \\
\hline 241-AZ-102 & 68.4 & 114.0 \\
\hline 241-AY-102/241-C-106 & 128.9 & 152.9 \\
\hline 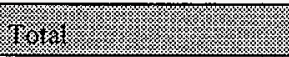 & $25 \% 2:$ & 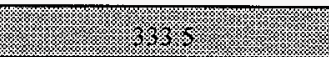 \\
\hline
\end{tabular}

${ }^{2}$ Based on 90 percent retrieval from 241-AZ-101, 60 percent retrieval from 241-AZ-102, and 85 percent retrieval from 241-AY-102/241-C-106 (Manuel et al. 1996).

As shown in Table G2-1, a large fraction of the available HLW feed for Phase I comes from 241-AY-102/241-C-106. Therefore, the HLW feed quantity is strongly dependent upon the ability to retrieve 241-C-106 in Project W-320.

\section{G2.5 W-211, "INITLAL WASTE RETRIEVAL SYSTEM"}

Project W-211 provides the in-tank processing systems required for 241-AZ-102 and 241-AY-102/241-C-106. The construction schedules must be consistent with the late need dates provided in Section 3.5 of the Tank Waste Remediation System Operation and Utilization Plan, (Kirkbride et al. 1997). 
HNF-SD-TWR-AGA-003

Revision 0

G3.0 REFERENCES

Boston, H. L., 1997a, Subcontract Number 80232764-9-K001, Interim Issue of Waste Feed Delivery Mid-Level Logics, letter to A. M. Umek, LMHC-9756006 (August 4), Lockheed Martin Hanford Corporation, Richland, Washington.

Boston, H. L., 1997b, Subcontract Number \#80232764-9-K001, Recommendation for Project W-314 Schedule Acceleration, letter to A. M. Umek, LMHC-9752990 (April 4), Lockheed Martin Hanford Corporation, Richland, Washington.

Claghorn, R. D., J. D. Galbraith, and T. B. Salzano (FDNW), 1997, Alternatives Generation and Analysis for the Phase I Intermediate Waste Feed Staging System Design Requirements, HNF-SD-TWR-AGA-001, Revision 1, Numatec Hanford Corporation, Richland, Washington.

DOE, 1996a, TWRS Privatization (BNFL, Inc.) Contract DE-RP06-96RL13308, September 1996, U.S. Department of Energy-Richland Operations Office, Richland, Washington.

DOE, 1996b, TWRS Privatization (LMAES) Contract DE-RP06-96RL13309, September 1996, U.S. Department of Energy-Richland Operations Office, Richland, Washington.

Kirkbride, R. A., G. K. Allen, P. J. Certa, A. F. Manuel, R. M. Orme, L. W. Shelton, E. J. Slaathaug, R. S. Wittman, G. T. MacLean (SESC), and D. L. Penwell (SESC), 1997, Tank Waste Remediation System Operation and Utilization Plan, HNF-SD-WM-SP-012, Revision 0, Numatec Hanford Corporation, Richland, Washington.

Manuel, A. F., S. L. Lambert, and G. E. Stegen, 1996, Phase I High-Level Waste Pretreatment and Feed Staging Plan, WHC-SD-WM-ES-370, Revision 1, Westinghouse Hanford Company, Richland, Washington. 
HNF-SD-TWR-AGA-003

Revision 0

This page intentionally left blank.

G-8 


\section{DISTRIBUTION SHEET}

\begin{tabular}{|c|c|c|c|c|c|}
\hline \multirow{2}{*}{$\begin{array}{l}\text { To } \\
\text { Distribution }\end{array}$} & \multirow{2}{*}{\multicolumn{3}{|c|}{$\begin{array}{l}\text { From } \\
\text { A. F. Manue1 }\end{array}$}} & \multicolumn{2}{|l|}{ Page 1 of 1} \\
\hline & & & & \multicolumn{2}{|c|}{ Date $9 / 29 / 97$} \\
\hline \multicolumn{4}{|c|}{ Project Title/Work Order } & \multicolumn{2}{|c|}{ EDT No. 622692} \\
\hline \multicolumn{4}{|c|}{$\begin{array}{l}\text { Alternatives Generation and Analysis for the Phase I High-Leve1 } \\
\text { Waste Pretreatment Process Selection, HNF-SD-TWR-AGA-003, Rev. } 0\end{array}$} & \multicolumn{2}{|l|}{ ECN No. } \\
\hline \multicolumn{2}{|c|}{ Name } & $\begin{array}{c}\text { Text } \\
\text { With All } \\
\text { Attach. }\end{array}$ & Text Only & $\begin{array}{l}\text { Attach./ } \\
\text { Appendix } \\
\text { Only }\end{array}$ & $\begin{array}{l}\text { EDT/ECN } \\
\text { On!y }\end{array}$ \\
\hline
\end{tabular}

Central files

DOE Reading Room

G. K. Allen

D. G. Baide

S. K. Baker

R. E. Bauer

A. L. Boldt

H. L. Boston

v. C. Boyles

A. F. Choho

R. D. Claghorn

T. W. Crawford

P. M. Daling

T. A. Flement

J. D. Galbraith

J. S. Garfield

K. A. Gasper

J. P. Harris

J. o. Honeyman

N. W. Kirch

R. A. Kirkbride

C. E. Leach

G. T. Maclean

A. F. Manuel (3 copies)

R. P. Marshall

R. H. Orme

I. G. Papp

D. L. Penwell

B. B. Peters

D. E. Place

R. D. Potter

R. W. Powell

W. E. Ross

E. J. Slaathaug

J. N. Strode

R. L. Treat

J. E. Van Beek

D. J. Washenfelder

$\underline{D Q E}$

R. Carreon

R. A. Gilbert

PNL

K. D. Wiemers

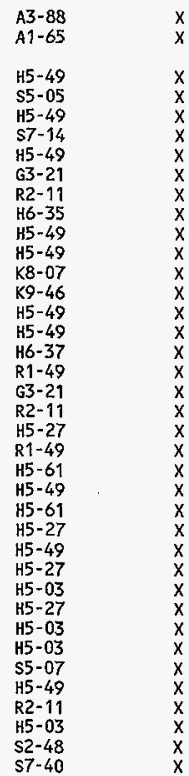

K6-51

K6-51

$x$

$x$

K6-51

$x$ 